\title{
Neovascular Age-Related Macular Degeneration in the Era of Value-Based Health Care
}

Citation for published version (APA):

Elshout, M. (2019). Neovascular Age-Related Macular Degeneration in the Era of Value-Based Health Care. [Doctoral Thesis, Maastricht University]. Gildeprint Drukkerijen.

https://doi.org/10.26481/dis.20190516me

Document status and date:

Published: 01/01/2019

DOI:

10.26481/dis.20190516me

Document Version:

Publisher's PDF, also known as Version of record

\section{Please check the document version of this publication:}

- A submitted manuscript is the version of the article upon submission and before peer-review. There can be important differences between the submitted version and the official published version of record.

People interested in the research are advised to contact the author for the final version of the publication, or visit the DOI to the publisher's website.

- The final author version and the galley proof are versions of the publication after peer review.

- The final published version features the final layout of the paper including the volume, issue and page numbers.

Link to publication

\footnotetext{
General rights rights.

- You may freely distribute the URL identifying the publication in the public portal. please follow below link for the End User Agreement:

www.umlib.nl/taverne-license

Take down policy

If you believe that this document breaches copyright please contact us at:

repository@maastrichtuniversity.nl

providing details and we will investigate your claim.
}

Copyright and moral rights for the publications made accessible in the public portal are retained by the authors and/or other copyright owners and it is a condition of accessing publications that users recognise and abide by the legal requirements associated with these

- Users may download and print one copy of any publication from the public portal for the purpose of private study or research.

- You may not further distribute the material or use it for any profit-making activity or commercial gain

If the publication is distributed under the terms of Article $25 \mathrm{fa}$ of the Dutch Copyright Act, indicated by the "Taverne" license above, 


\section{Neovascular Age-Related Macular Degeneration in the Era of Value-Based Health Care}


Cover design and layout: (c) evelienjagtman.com

Printed by: Gildeprint Enschede

ISBN 9789463235556

(C) 2019 Mari Elshout, Eindhoven

All rights reserved. No part of this thesis may be reproduced or transmitted in any form or by any means, electronic or mechanical, including photocopying, recording or any information storage or retrieval system, without permission in writing from the author, or, when appropriate, from the publishers of the publications. 


\title{
Neovascular Age-Related Macular Degeneration in the Era of Value-Based Health Care
}

\author{
Proefschrift
}

\author{
ter verkrijging van de graad van doctor aan de Universiteit Maastricht, \\ op gezag van de Rector Magnificus, prof. dr. mr. Rianne M. Letschert \\ volgens het besluit van het College van Decanen, \\ in het openbaar te verdedigen \\ op donderdag 16 mei 2019 om 14:00 uur
}

door

Mari Elshout

Geboren op 3 april 1985 te Eindhoven 


\section{Promotor}

Prof. dr. C.A.B. Webers

\section{Copromotor}

Dr. J.S.A.G. Schouten (CWZ Nijmegen)

\section{Beoordelingscommissie}

Prof. dr. A.E.R.C.H. Boonen (voorzitter)

Prof. dr. M.A. Joore

Prof. dr. J.E.E. Keunen (Radboudumc Nijmegen)

Prof. dr. G.G. van Merode

Dr. F.D. Verbraak (Academisch Medisch Centrum Amsterdam)

Het onderzoek in dit proefschrift kwam mede tot stand dankzij een beurs van ZonMw. 


\section{Table of contents}

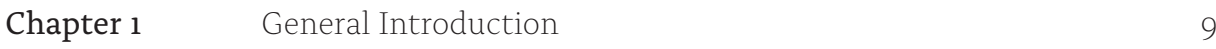

Chapter 2 Tracing the natural course of visual acuity and quality of

life in neovascular age-related macular degeneration: a

systematic review and quality of life study

Chapter 3 A systematic review on the quality, validity and usefulness

61 of current cost-effectiveness studies on the treatment for neovascular age-related macular degeneration

Chapter 4 The cost-utility of aflibercept for the treatment of agerelated macular degeneration compared to bevacizumab and ranibizumab and the influence of model parameters

Chapter 5 A new epidemiological aid in deciding whether to continue or stop a treatment

Chapter 6 Distinguishing between better and worse visual acuity by 121 studying the correlation with quality of life in neovascular age-related macular degeneration

Chapter $7 \quad$ A systematic approach to evaluate practice based processand outcome data applied to the treatment of neovascular age-related macular degeneration

Chapter 8

Discussion

Chapter 9

Summary 167

Samenvatting

Addendum

Valorisatie

Dankwoord 191

Curriculum Vitae 195

List of publications 
4.
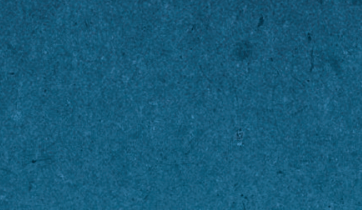

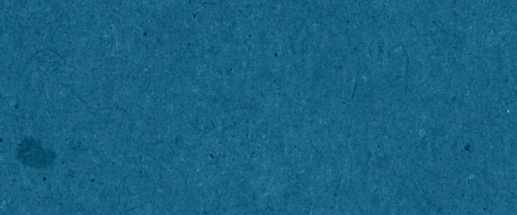
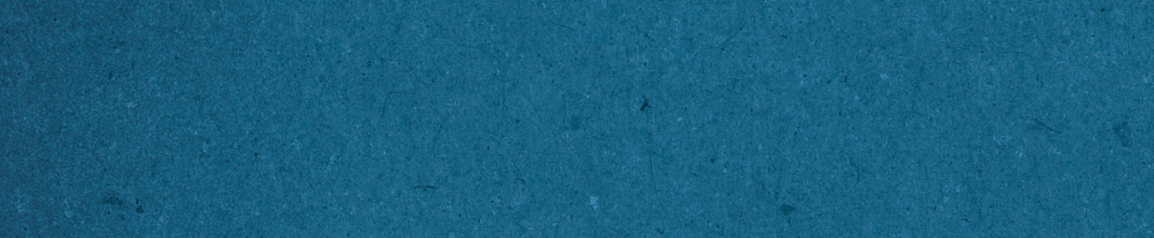

8

$y 3$

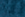


Chapter 1

General Introduction 


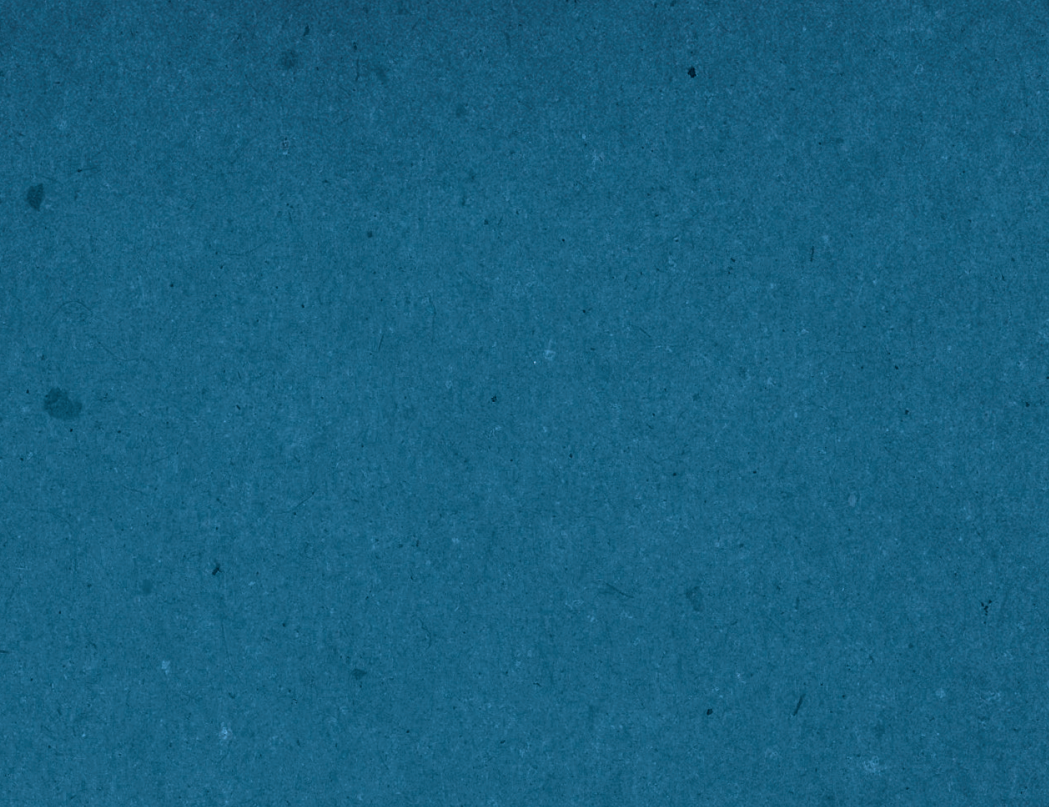




\section{Age-related macular degeneration}

Age-related macular degeneration (AMD) is a degenerative eye disease in elderly individuals. It affects the central part of the retina, the macula (Figure 1). The macula is responsible for the visual acuity and colour vision. As its name implies, the prevalence of AMD increases with age. It can develop from early stages into a more severe stage leading to a gradual loss of visual acuity.

Clinically, the presence of drusen in the macula is a hallmark of the disease (Figure 2). Histologically, drusen are deposits of extracellular material between Bruch's membrane and the retinal pigment epithelium (RPE) (Figure 3).1-4 Their presence in the retina predisposes the eye to develop late-stage AMD, with neovascular lesions or geographic atrophy. 5 , 6 The appearance of drusen in the macula has a role in diagnosing and classifying AMD.

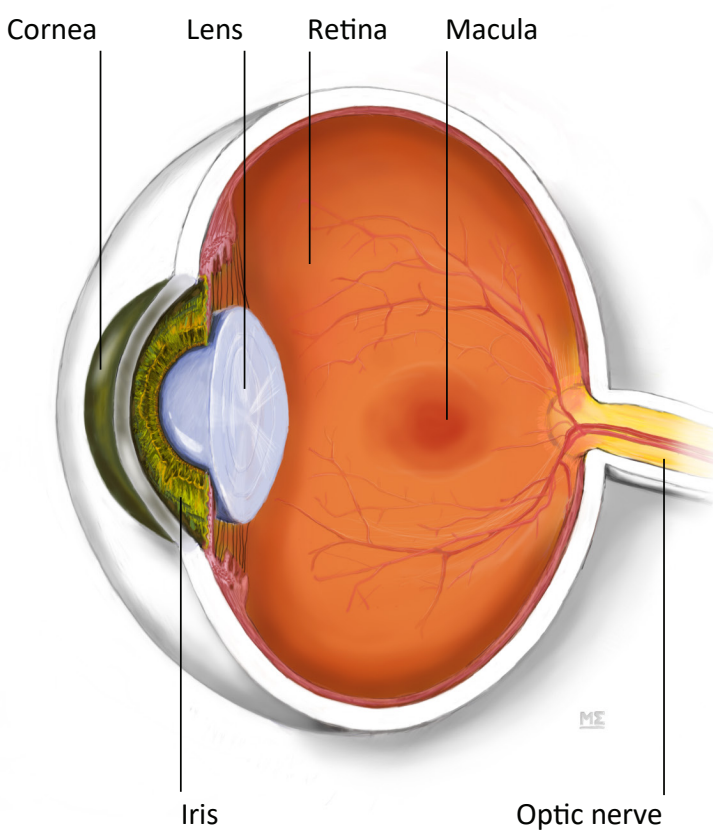

Figure 1. Cutaway view of the eye with the relative position of the macula and other structures. 


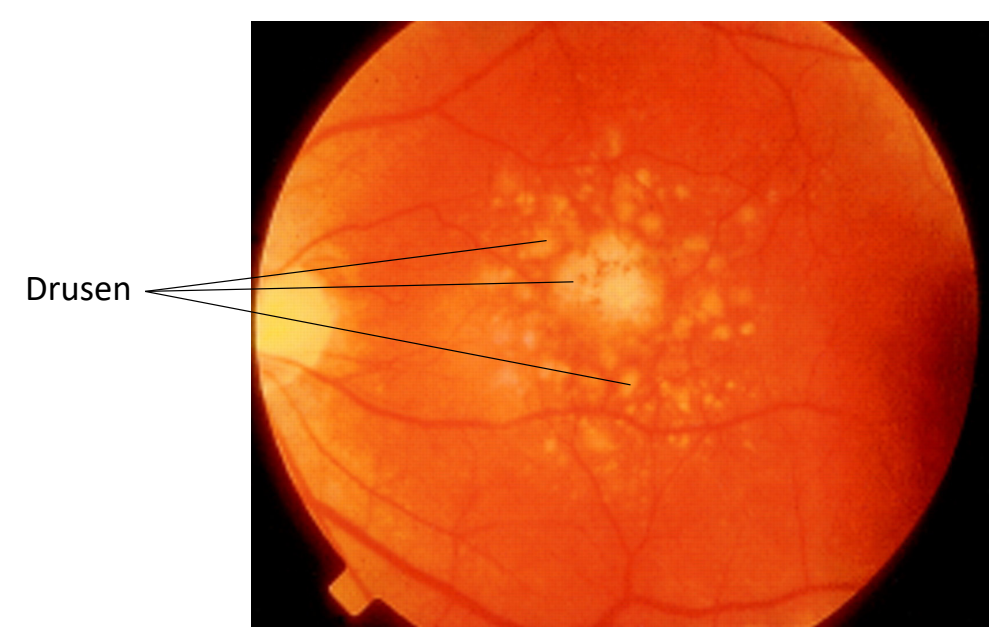

Figure 2. Color fundus photograph showing macular drusen. Image from Taylor et al. BMJ 2002, 325.

AMD can be classified into early AMD, intermediate AMD, and late AMD, based on the appearance of the macula? Fundus lesions found within two disc diameters of the fovea are regarded as relevant for classification. Persons with small drusen $(<63 \mu \mathrm{m})$ have normal aging changes of the macula with no clinically relevant risk of developing late AMD. Early AMD is defined by medium-sized drusen $(\geq 63-<125 \mu \mathrm{m})$, without pigmentary abnormalities thought to be related to AMD. Intermediate AMD is defined by large drusen or pigmentary abnormalities combined with the presence of at least mediumsized drusen. The term late AMD is reserved for neovascular AMD (nAMD), based on the presence of a choroidal neovascularisation (CNV), or for geographic atrophy (GA)? 


\section{Epidemiology}

The disease is the leading cause of irreversible severe vision loss and blindness in highincome countries in people over 50 years of age., ${ }^{8,9}$ The incidence is increasing over time, mainly because of the aging of the population. ${ }^{10}$ Worldwide, the projected number of people with any form of AMD is around 196 million in 2020, expected to increase to 288 million in 2040. The number of people with late AMD (nAMD or GA), is expected to increase from 11 million in 2020 to 20 million in 2040." Important risk factors for AMD are age, smoking, familial predisposition, genetic changes, and female gender. ${ }^{12}$ Having AMD in one eye significantly raises the risk of developing AMD in the other eye.13 While GA is more prevalent, nAMD has a poorer prognosis when left untreated and is the cause of legal blindness due to AMD in the majority of untreated patients. ${ }^{14}$ 


\section{Pathogenesis}

The pathogenesis of AMD is complex and multifactorial, and not fully understood. The presence of drusen in combination with a genetic predisposition contributes to the development of late AMD.

Several mechanisms and pathways in the development and progression of the disease have been described. Specifically, immunological pathways are thought to play an important role. The alternative pathway of the complement system, part of the innate immune system, is thought to be a pathway involved in AMD.15,16 Secondly, the association of cardiovascular risk factors with AMD and the accumulation of cholesterol and lipids in drusen suggest the high-density lipoprotein pathway is implicated; several genes involved in this pathway have also been associated with AMD., ${ }^{3,6}$ Thirdly, a role for the extracellular matrix (ECM) pathway is suggested by genetic associations. ${ }^{17-20}$ Bruch's membrane, a histologically important site in the pathogenesis of AMD, is an important ECM complex. ${ }^{21} \mathrm{~A}$ fourth pathway, implicated in the occurrence CNV in nAMD, is the angiogenesis signalling pathway. In this pathway, the vascular endothelial growth factor (VEGF)-A protein is a cytokine that promotes angiogenesis and vascular permeability. It is an important factor that promotes neovascularisation in nAMD. ${ }^{22-24}$ The precise mechanisms of developing CNV are not well understood. Any pathologic process that involves the RPE and damages the Bruch membrane can be complicated by CNV, which may be considered as a wound healing response to an insult of the RPE. An RPE-derived protein, pigment epithelium derived factor (PEDF), was found to have an inhibitory effect on ocular neovascularisation. ${ }^{25}$ The balance between anti-angiogenic factors and angiogenic factors such as VEGF-A is thought to determine the growth of $\mathrm{CNV}^{26,27}$

Other pathological features of nAMD include serous or haemorrhagic detachment of the RPE and vitreous haemorrhage. Pathological changes may ultimately result in a disciform granulomatous scar that replaces the normal architecture of the outer retina that leads to permanent loss of vision (Figure 3). 


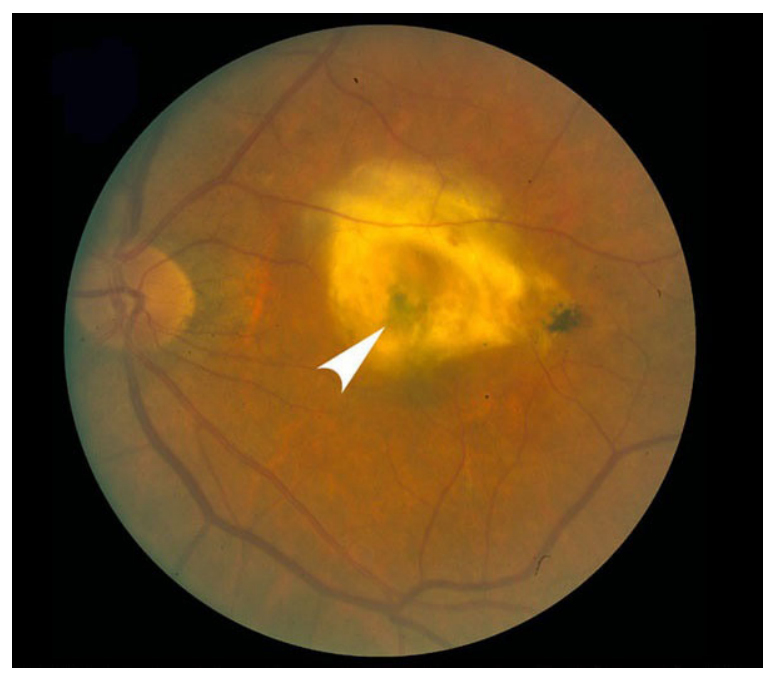

Figure 3. Color fundus photograph from a person with end-stage nAMD. A large disciform scar (arrowhead) covering the macular region is visible. Image from webvision.med.utah.edu. 


\section{Detection of CNV in nAMD}

CNV may cause severe central vision loss in nAMD. CNV is an ingrowth of new blood vessels from the choriocapillaris into the sub-RPE space through a defect in the outer layer of Bruch's membrane, gaining access to the inner collagenous layer and/or subretinal space (see Figure 4). Within this space, the CNV can leak fluid and blood and may be accompanied by a serous or hemorrhagic detachment of the RPE. In addition, fibrovascular tissue may grow within Bruch's membrane or between the neurosensory retina and the RPE, ultimately leading to a disciform scar that leads to permanent loss of central vision.

A comprehensive ocular examination is needed for detecting and diagnosing CNV in nAMD. Fundoscopy may show elevation of the RPE, presence of subretinal or intraretinal fluid or blood, hard exudates, pigment epithelial detachment, and RPE tears. Occasionally, the oval gray-green CNV lesion itself is visible (Figure 5).

Fluorescein angiography (FA) allows to study the circulation of the retina and choroid. Photographs of the retina are taken after intravenous injection of sodium fluorescein. FA patterns of CNV vary and include classic and occult CNV. Classic CNV is an area of bright, fairly rapidly occuring hyperfluorescence seen in the early phase of choroidal filling in the FA study, with leakage of dye obscuring the boundaries of this area in the late phases (Figure 5). Occult CNV is an area of which the entire extent may be difficult to define because the leakage is difficult to detect, or because the hyperfluorescence and leakage are partially blocked, either by a serous detachment of the RPE, subretinal blood, turbid fluid or pigment.

FA is the gold standard for diagnosing CNV. In cases with some overlying blood, or occult $\mathrm{CNV}$, indocyanine green (ICG) angiography may better demonstrate the vascular lesion.

Optical coherence tomography (OCT) is a non-invasive, noncontact imaging technique that produces cross-sectional images of ocular tissue. In the diagnosis and follow-up of nAMD, it is used to detect macular intra- or subretinal fluid, which suggests active leakage of a CNV (Figure 5). A newer imaging technique is optical coherence tomography angiography (OCTA). It employs motion contrast imaging to high-resolution volumetric blood flow information generating angiographic images.

With FA and OCT, different types of CNV in nAMD are distinguished: type 1 CNV, which occurs within the sub-RPE space, and corresponds to angiographically occult CNV; type $2 \mathrm{CNV}$, which occurs within the subretinal space, and corresponds to angiographically classic CNV; and type $3 \mathrm{CNV}$, intraretinal retinal angiomatous proliferation. ${ }^{28-30}$ 


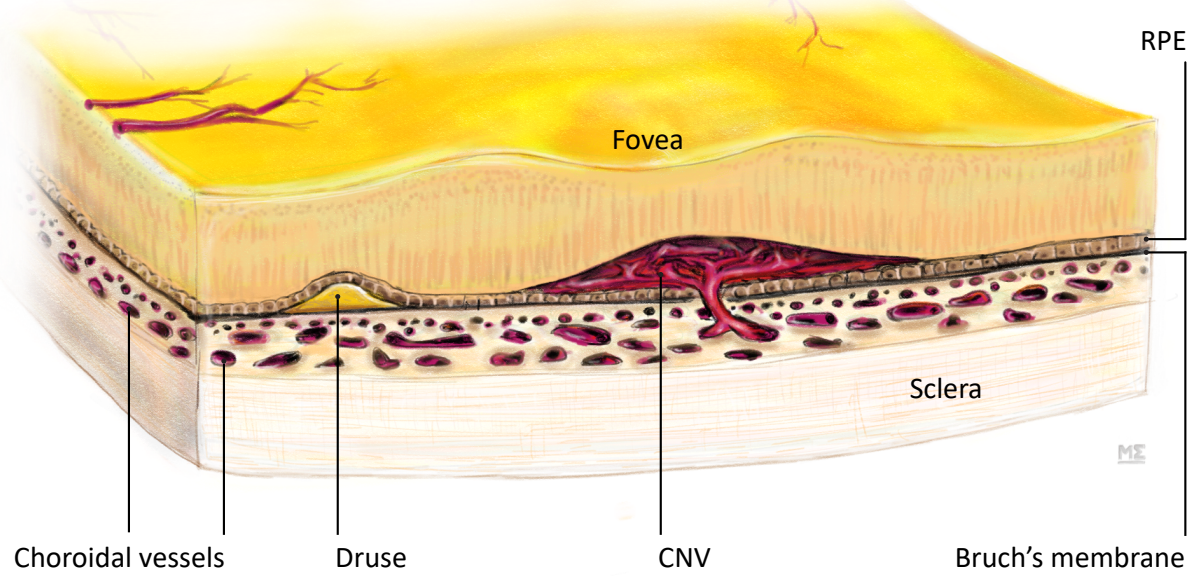

Figure 4. Schematic of choroidal neovascularisation with ingrowth through Bruch's membrane into the subretinal space. $C N V$, choroidal neovascularisation; $R P E$, retinal pigment epithelium.

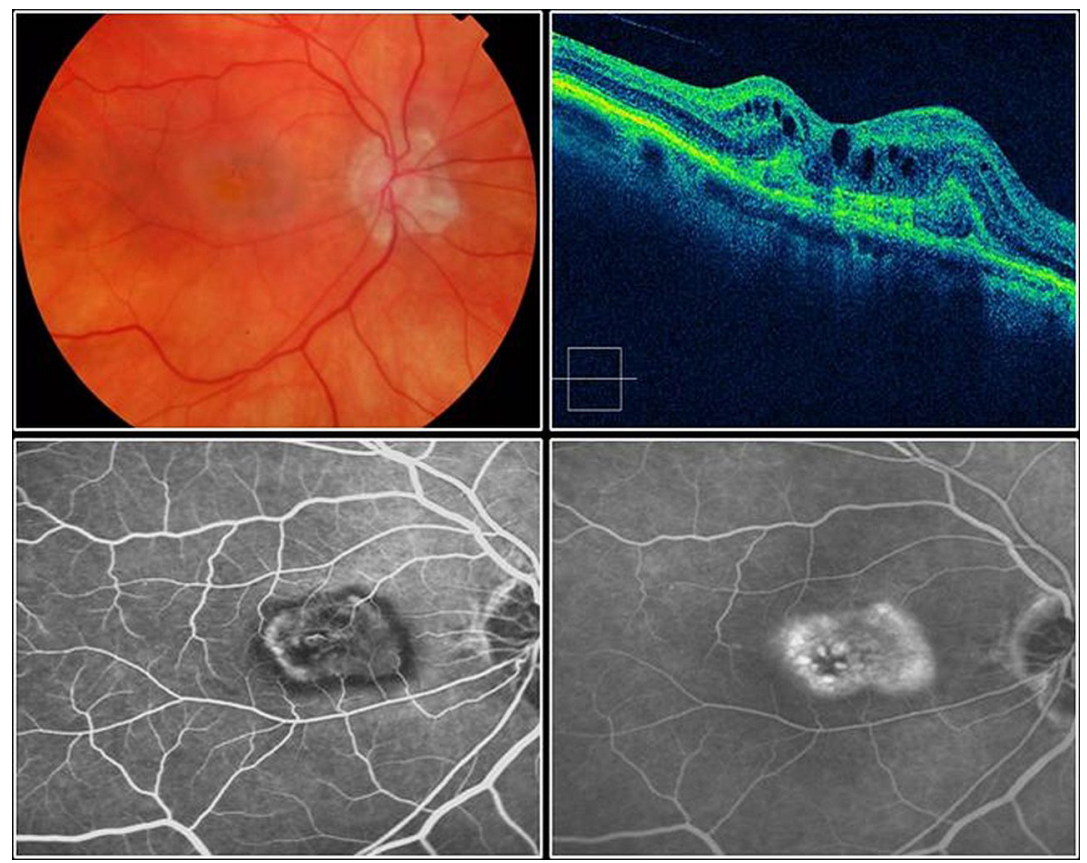

Figure 5. Imaging of classic choroidal neovascularisation (CNV). Top left: Color fundus photograph showing grey-green lesion at the fovea. Top right: Spectral domain optical coherence tomograph (OCT) showing intraretinal oedema as a result of the CNV. Bottom left: the neovascular net is delineated with distinct margins in early-phase fluorescein angiography (FA). Bottom right: Leakage in late-phase Leakage in latephase FA confirms the biologic activity of the CNV. Image from Schmidt-Erfurth et al, BJO 2014;98, under licence. 


\section{Symptoms of nAMD}

Patients with nAMD may have symptoms such as blurred vision or gray or dark areas in their central vision and/or a distortion of the image. Neovascular age-related macular degeneration does not cause visual field loss. The disease usually begins in one eye, with a high risk of affecting the other eye later. Untreated nAMD leads to permanent loss of central vision. 


\section{Impact on quality of life}

A decreasing visual acuity over time leads to worsening of vision-related functions, and more anxiety and depression. ${ }^{31}$ Several studies have focused on quality of life (QoL) in nAMD patients. These studies have shown that nAMD causes a substantial decrease in QoL which is highly dependent on the degree of vision loss in the better-seeing eye. ${ }^{32-36}$ As an example of measuring QoL on the time-trade-off scale, one study found that the average person with a 0.5 Snellen visual acuity in the better-seeing eye was willing to trade two of every ten years of life with 0.5 Snellen visual acuity in return for perfect vision (utility value of 0.80 ), while the average person with counting fingers vision in the better eye was willing to trade approximately five of every ten remaining years of life (utility value of 0.52) in return for perfect vision. ${ }^{36}$ Such values can be compared to other diseases. For example, persons with diabetes valued their QoL with a score of 0.78 , those with colorectal cancer, 0.78 ; and those with multiple sclerosis, $0.64 .{ }^{37,38}$ 


\section{Treatments}

A number of treatments for $\mathrm{nAMD}$ are available such as thermal laser photocoagulation, photodynamic therapy, medications and macular surgery.39-41

Thermal laser photocoagulation reduces the risk of severe vision loss in patients with CNV secondary to nAMD. ${ }^{42,43}$ Drawbacks include loss of visual acuity as a result of damage to the neurosensory retina and the RPE. Furthermore, benefit is limited due to persistence or recurrence of CNV. From the 1980s through 2001, laser treatment was the primary treatment of classic CNV and CNV with classic and occult components, in which the lesion boundary was well demarcated. Compared to thermal laser photocoagulation, drugbased therapies are more beneficial.40,42,44 With the advent of photodynamic therapy and anti-VEGF therapies, thermal laser photocoagulation has become rarely used anymore in the treatment of nAMD.45 Laser photocoagulation remains an option for extrafoveal lesions when the clinician believes it can be safely applied.

Macular surgery for the treatment of CNV in NAMD has been tried in multiple series, thus far with little clinical success. ${ }^{4,46}$

The introduction of photodynamic therapy (PDT) in 2000 added a treatment option that was less-destructive to retinal tissue than thermal laser treatment. It has been shown to be of clinical benefit for the treatment of nAMD, especially in eyes with classic subfoveal $\mathrm{CNV}^{47}$ It is a 2-step process that involves the systemic administration of a photosensitising drug (verteporfin) followed by an application of laser light to the affected tissue to incite a localised photochemical reaction. This reaction generates reactive oxygen species that can lead to capillary endothelial cell damage and vessel thrombosis. The gain in visual acuity with PDT was still limited and only entailed slowing of the vision loss over time. ${ }^{48}$ Subgroup analysis in the Treatment of AMD with Photodynamic Therapy (TAP) investigation revealed that participants with predominantly classic CNV (meaning that the area of classic CNV occupied $\geq 50 \%$ of the entire lesion) experienced the greatest benefit. ${ }^{49}$ With the advent of anti-VEGF treatment, the use of PDT in nAMD has been reduced dramatically.

Inhibition of vascular endothelial growth factor (VEGF) via intravitreal injection (Figure 6) is the most successful therapy for nAMD with better visual outcomes compared to other treatments with a gain in visual acuity that had not been achieved previously.5 In 2004, the anti-VEGF drug pegaptanib was approved by the United States Food and Drug Administration (FDA). It was the first intravitreal anti-angiogenic drug to become available for the treatment of nAMD. The VEGF Inhibition Study in Ocular 
Neovascularization (VISION) trial enrolled 1196 patients receiving pegaptanib or sham injections every 6 weeks. Seventy per cent of the treated patients lost fewer than three lines of vision and ten per cent lost more than six lines at twelve months, compared to $55 \%$ and $22 \%$, respectively, among patients who received sham injections. Pegaptanib therapy does not improve visual acuity and does not prevent disease progression. In general, patients still lose vision with pegaptanib, and it is rarely used today as newer anti-VEGF drugs with better efficacy have been developed.

Bevacizumab is an anti-VEGF antibody that was approved by the FDA in 2004 for the treatment of metastatic colorectal cancer. It is not licensed for the treatment of nAMD. Despite its off-label designation, the use of intravitreal bevacizumab for nAMD is widespread..$^{51}$ The Comparison of Age-Related Macular Degeneration Treatments Trials (CATT) was a randomised clinical trial to assess the relative safety and efficacy of ranibizumab and bevacizumab for the treatment of subfoveal nAMD. The final 2-year results showed comparable efficacy of ranibizumab and bevacizumab on visual acuity.52

Ranibizumab is an anti-VEGF antibody specifically for intravitreal injection. The Minimally Classic/Occult Trial of the Anti-VEGF Antibody Ranibizumab in the Treatment of Neovascular AMD (MARINA) study showed that with ranibizumab administration every 4 weeks for 24 months, almost $40 \%$ of the ranibizumab treated patients experienced visual acuity improvement of 15 letters (translatable to e.g. Snellen visual acuity improving from 0.2 to 0.4 ) or more as compared to sham treated patients. ${ }^{53}$ The Anti-VEGF antibody for the treatment of predominantly classic choroidal neovascularisation in AMD (ANCHOR) study found that visual acuity improved 15 letters or more in $34 \%$ to $41 \%$ of patients with predominantly classic CNV after 24 months. ${ }^{54}$ The effect of ranibizumab in terms of change in visual acuity has been found to be comparable to bevacizumab in other studies. $^{52,55}$

Aflibercept is the latest anti-VEGF available for use in nAMD. Its efficacy was found to be comparable to ranibizumab. ${ }^{56}$ In the VIEW 1 and 2 (VEGF Trap-Eye: Investigation of Efficacy and Safety in Wet Age-Related Macular Degeneration 1 and 2) studies, patients received $2 \mathrm{mg}$ aflibercept as monthly or bimonthly regimens, or ranibizumab $0.5 \mathrm{mg}$ monthly regimens. All aflibercept and ranibizumab regimens were equally effective in improving visual acuity at 2 years follow-up. The bimonthly aflibercept group was similar to ranibizumab in visual acuity outcome at 2 years, but with slightly fewer injections needed. ${ }^{57}$ Although this was due to the protocol defining bimonthly injections for aflibercept and monthly injections for ranibizumab in the first year, a third year of follow up, using an as-needed protocol, showed again that slightly fewer injections were needed in the aflibercept groups versus the ranibizumab group..$^{56}$ 
CHAPTER 1

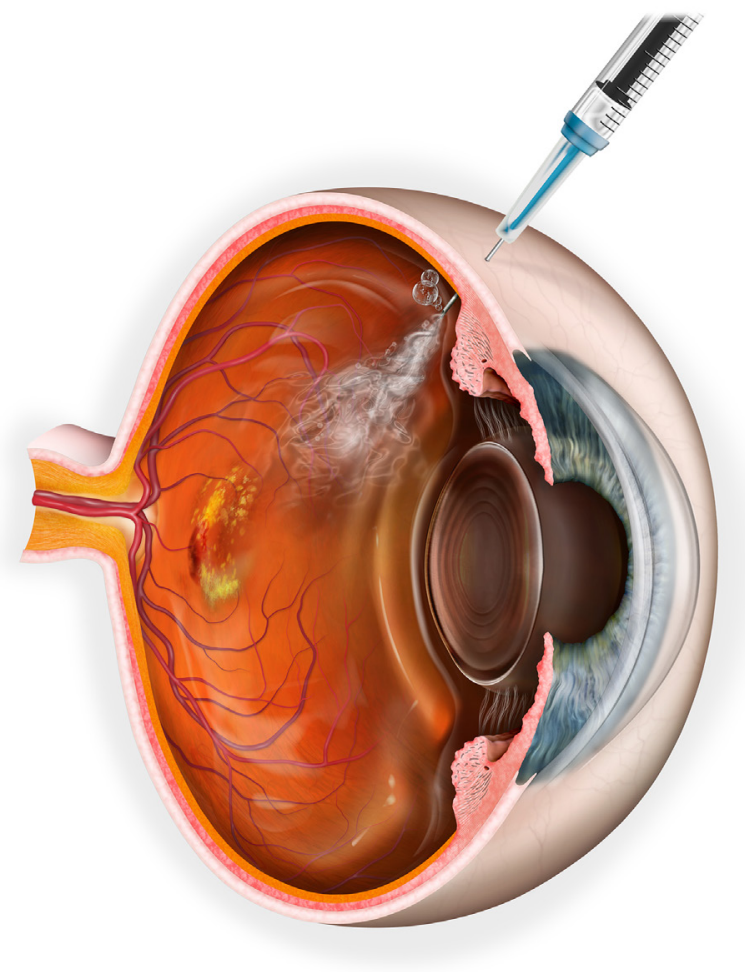

Figure 6. Intravitreal administration of a drug. Image courtesy of Mark Erickson (Jirehdesign.com).

The treatment with anti-VEGFs requires a regular monitoring of patients with OCT and fundoscopy to detect recurrence of a $\mathrm{CNV}$ at an early stage and prevent visual acuity loss due to treatment delay. This leads to a mean number of regular monitoring visits and visits for injection of about 6 to 8 per year..$^{58-60}$ 


\section{Macular degeneration in the context of value-based health care}

"Vaccines. Anesthesia. Penicillin. Bypass surgery. Decoding the human genome. Unquestionably, all are life-saving medical breakthroughs. But one breakthrough that will change the face of medicine is being slowed by criticism, misunderstanding, and a reluctance to do things differently. That breakthrough is value-based care, the goal of which is to lower health care costs and improve quality and outcomes."61 In this citation, Toby Cosgrove, MD, a former heart surgeon, president and CEO of the Cleveland Clinic, expands on "value-based health care" with the components quality of care and costs.

Value-based health care is a concept in health care reform aimed at maximising the value for the patient. Michael E. Porter was one of the first to define the concept and stated that the purpose of the health care system is not to minimise costs but to deliver value to patients, that is, better health per dollar spent.62-64 A value-based system is based on 3 principles: (1) the goal is value for patients, (2) care delivery is organised around medical conditions and care cycles, and (3) results are measured. ${ }^{64}$

Value-Based health care is becoming intricately more involved in the current health care system. In $\mathrm{nAMD}$, several factors demand a value-based approach to the treatment of patients. The number of patients with nAMD is expected to increase due to high prevalence in elderly patients and the increasing elderly population. ${ }^{65}, 66$ Highly effective but costly new treatments have become available. The budgets to treat patients are constrained, yet patients require several treatments per year for many years. Although most anti-VEGFs are costly, they may still be cost-effective. In order to maximise the patient value per euro spent on nAMD treatment, cost-effectiveness analysis provides a tool to define the most attractive treatment strategies.

In daily practice, any choice in the management of patients with $n A M D$ has implications for the outcome as well as the costs. Balancing both costs and outcome by means of decisions that are made in the process of care is essential in addressing value-based health care. Decisions concern whether nAMD should be treated at all, the choice of the drug to start with, cost-effectiveness of treatment strategies and clinical decisions on changing or stopping treatment. Moreover, monitoring process and outcome in daily practice may change future decisions based on decisions made in the past.

One of the pillars of value-based health care is measuring results. A health care system should move towards objectively measuring the patients' overall benefit, integrating all benefits and adverse events, conferred by healthcare interventions. To this end, it is 
necessary to know the objective patient value associated with the medical services that healthcare budgets are purchasing. In a value-based system, it is necessary to provide the highest-quality, most cost-effective treatment. This requires including opinions of patients about the QoL associated with their condition(s) and treatment(s): patient value, the most important pillar in value-based medicine. In maximising patient value, one should aim to treat as many patients as possible with the treatment indicated for these patients. Once this is accomplished, the financial value becomes important.

\section{Patient value}

The gain in patient value associated with a healthcare intervention may be defined by the improvement it confers in QoL and/or gain in life expectancy. Gain in life expectancy associated with interventions can easily be assessed from clinical trials and other studies where interventions are compared. Changes in QoL associated with diseases and health states (one or more diseases) are much more complex to measure.

This has led to a large number of different measures that have been developed to measure QoL in patients. A range of questionnaires can be used to measure and quantify QoL. A questionnaire can be disease-specific, such as the MacDQoL measure to assess the impact of macular degeneration. ${ }^{67}$ A QoL questionnaire that translates several domains of the visual functioning in daily life into one QoL score, is less specific for one eye disease, more generic for several eye diseases, and can be used to compare QoL between eye diseases. An example of such a measure is the NEI-VFQ.52 An even more generic questionnaire that measures health-related QoL reflects more the general physical and mental health states of individuals. This enables comparisons between different diseases. Such questionnaires, valued and validated by a random sample of the general population, can be used to assess the utility measure. Such a questionnaire is especially valuable in cost-effectiveness- and cost-utility studies. An example is the Health Utilities Index Issue 3 (HUI-3) measure. ${ }^{68}$

\section{Financial Value}

In order to achieve the best outcome in patients, the most effective intervention needs to be selected. Specifically, when patient value between treatments is similar, costs determine the treatment scenario. ${ }^{69}$ Financial value includes the direct medical costs associated with an intervention, as well as the overall societal costs associated with that intervention. Included among the societal costs are the direct medical costs, the direct nonmedical costs (caregiver costs) and indirect medical costs (employment costs). The inclusion of societal costs generally makes an intervention more cost-effective. Essentially, cost-effectiveness analysis is the combination of patient value and financial value in a single analysis. In such analysis, standardisation is critical. Without standardisation, costutility analyses cannot be compared. 


\section{Outline of the thesis}

The treatment of nAMD was investigated from a value-based health care perspective. The research conducted is described in the next six chapters of this thesis. Chapters 2 through 4 take a population-level approach to address the need to treat patients with nAMD and the cost-effectiveness of doing so.

In chapter 2, therefore, the natural course of visual acuity and QoL in nAMD is traced and modelled based on visual acuity data from the literature and combined with QoL data from our QoL study. This is needed as a foundation for clinical decisions as to whether or not to treat patients, and to inform them about the expected outcome without treatment. These data are also needed for the economic models to assess the cost-effectiveness of treatments.

In chapter 3, we assess the quality and usefulness of published cost-effectiveness studies for the treatment of $\mathrm{nAMD}$, to underpin health policy decisions to decide on whether to allocate budget to treat patients and to select the most cost-effective treatment. It also shows the quality of cost-effectiveness analyses in this field that form the basis of the quality of health policy decisions.

In chapter 4, we report on the cost-effectiveness of different treatments for nAMD, with a better cost-effectiveness model based upon the outline of shortcomings reported in chapter 3 .

The next two chapters take a more individual and clinical approach in supporting decision making. Chapter 5 concerns a new method to assess whether a treatment has been effective for an individual patient, and thus support the decision as to whether this treatment should be continued or not. This has an impact both on the outcome and on the costs. Stopping an ineffective treatment saves resources and changing to a more expensive one increases costs, but could save resources when effective in reducing the risk of blindness and its costs.

Chapter 6 also focuses on supporting clinical decisions, by identifying the cutoff value in visual acuity below which a change in visual acuity (due to a treatment or due to natural progression) does not imply a further change in QoL. This would imply a focus to keep the visual acuity above this level to improve QoL and discuss the need for further treatment with the patient below this level. The result of such a discussion could be to stop a treatment that does not impact QoL. This reduces risks associated with treatments and saves resources. Moreover, the use of expensive 
drugs and regular follow-up visits that are a burden to the patients and the health care system can be reduced.

In chapter 7, data from daily practice were analysed to study the process and outcome of the treatment of patients with nAMD. These analyses are valuable in improving the quality, outcome and efficiency of care. Such analyses are especially valuable in the era of value-based care and the opportunities offered by the presence of electronic medical records and registrations. 


\section{References}

1. Al-Hussaini H, Schneiders M, Lundh P, Jeffery G. Drusen are associated with local and distant disruptions to human retinal pigment epithelium cells. Experimental eye research 2009;88:610-612.

2. Anderson DH, Mullins RF, Hageman GS, Johnson LV. A role for local inflammation in the formation of drusen in the aging eye. Am J Ophthalmol 2002;134:411-431.

3. Curcio CA, Presley JB, Malek G, Medeiros NE, Avery DV, Kruth HS. Esterified and unesterified cholesterol in drusen and basal deposits of eyes with age-related maculopathy. Experimental eye research 2005;81:731-741.

4. Rudolf M, Clark ME, Chimento MF, Li CM, Medeiros NE, Curcio CA. Prevalence and morphology of druse types in the macula and periphery of eyes with age-related maculopathy. Invest Ophthalmol Vis Sci 2008;49:1200-1209.

5. Wang JJ, Rochtchina E, Lee AJ, et al. Ten-year incidence and progression of age-related maculopathy: the blue Mountains Eye Study. Ophthalmology 2007;114:92-98.

6. Klein R, Klein BE, Knudtson MD, Meuer SM, Swift M, Gangnon RE. Fifteen-year cumulative incidence of age-related macular degeneration: the Beaver Dam Eye Study. Ophthalmology 2007;114:253-262.

7. Ferris FL, 3rd, Wilkinson CP, Bird A, et al. Clinical classification of age-related macular degeneration. Ophthalmology 2013;120:844-851.

8. Klein R, Klein BE, Jensen SC, Mares-Perlman JA, Cruickshanks KJ, Palta M. Age-related maculopathy in a multiracial United States population: the National Health and Nutrition Examination Survey III. Ophthalmology 1999;106:1056-1065.

9. Bunce C, Xing W, Wormald R. Causes of blind and partial sight certifications in England and Wales: April 2007-March 2008. Eye (Lond) 2010;24:1692-1699.

10. Resnikoff S, Pascolini D, Etya'ale D, et al. Global data on visual impairment in the year 2002. Bull World Health Organ 2004;82:844-851.

11. Wong WL, Su X, Li X, et al. Global prevalence of age-related macular degeneration and disease burden projection for 2020 and 2040: a systematic review and meta-analysis. The Lancet Global health 2014;2:e106-116.

12. Tomany SC, Wang JJ, Van Leeuwen R, et al. Risk factors for incident age-related macular degeneration: pooled findings from 3 continents. Ophthalmology 2004;111:1280-1287.

13. Lechanteur YT, van de Ven JP, Smailhodzic D, et al. Genetic, behavioral, and sociodemographic risk factors for second eye progression in age-related macular degeneration. Invest Ophthalmol Vis Sci 2012;53:5846-5852.

14. Jager RD, Mieler WF, Miller JW. Age-related macular degeneration. N Engl J Med 2008;358:2606-2617.

15. Ambati J, Atkinson JP, Gelfand BD. Immunology of age-related macular degeneration. Nature reviews Immunology 2013;13:438-451.

16. Anderson DH, Radeke MJ, Gallo NB, et al. The pivotal role of the complement system in aging and age-related macular degeneration: hypothesis re-visited. Progress in retinal and eye research 2010;29:95-112.

17. Chen W, Stambolian D, Edwards AO, et al. Genetic variants near TIMP3 and high-density lipoproteinassociated loci influence susceptibility to age-related macular degeneration. Proceedings of the National Academy of Sciences of the United States of America 2010;107:7401-7406.

18. Fiotti N, Pedio M, Battaglia Parodi M, et al. MMP-9 microsatellite polymorphism and susceptibility to exudative form of age-related macular degeneration. Genetics in medicine : official journal of the American College of Medical Genetics 2005;7:272-277. 
19. Fritsche LG, Igl W, Bailey JN, et al. A large genome-wide association study of age-related macular degeneration highlights contributions of rare and common variants. Nature genetics 2016;48:134-143.

20. Liutkeviciene R, Lesauskaite V, Sinkunaite-Marsalkiene G, et al. The Role of Matrix Metalloproteinases Polymorphisms in Age-Related Macular Degeneration. Ophthalmic genetics 2015;36:149-155.

21. Johnson LV, Anderson DH. Age-related macular degeneration and the extracellular matrix. New England Journal of Medicine 2004;351:320-322.

22. Dvorak HF, Brown LF, Detmar M, Dvorak AM. Vascular permeability factor/vascular endothelial growth factor, microvascular hyperpermeability, and angiogenesis. The American journal of pathology 1995;146:1029-1039.

23. Kliffen M, Sharma HS, Mooy CM, Kerkvliet S, de Jong PT. Increased expression of angiogenic growth factors in age-related maculopathy. Br J Ophthalmol 1997;81:154-162.

24. Senger DR, Galli SJ, Dvorak AM, Perruzzi CA, Harvey VS, Dvorak HF. Tumor cells secrete a vascular permeability factor that promotes accumulation of ascites fluid. Science (New York, NY) 1983;219:983985.

25. Bhutto IA, McLeod DS, Hasegawa T, et al. Pigment epithelium-derived factor (PEDF) and vascular endothelial growth factor (VEGF) in aged human choroid and eyes with age-related macular degeneration. Experimental eye research 2006;82:99-110.

26. Campochiaro PA, Soloway P, Ryan SJ, Miller JW. The pathogenesis of choroidal neovascularization in patients with age-related macular degeneration. Molecular vision 1999;5:34.

27. Wang H, Hartnett ME. Regulation of signaling events involved in the pathophysiology of neovascular AMD. Molecular vision 2016;22:189-202.

28. Farecki ML, Gutfleisch M, Faatz H, et al. Characteristics of type 1 and 2 CNV in exudative AMD in OCT-Angiography. Graefes Arch Clin Exp Ophthalmol 2017;255:913-921.

29. Freund KB, Zweifel SA, Engelbert M. Do we need a new classification for choroidal neovascularization in age-related macular degeneration? Retina 2010;30:1333-1349.

30. Freund KB, Ho IV, Barbazetto IA, et al. Type 3 neovascularization: the expanded spectrum of retinal angiomatous proliferation. Retina 2008;28:201-211.

31. Soubrane G, Cruess A, Lotery A, et al. Burden and health care resource utilization in neovascular agerelated macular degeneration: findings of a multicountry study. Arch Ophthalmol 2007;125:1249-1254.

32. Brown GC, Brown MM, Campanella J, Beauchamp GR. The cost-utility of photodynamic therapy in eyes with neovascular macular degeneration--a value-based reappraisal with 5-year data. Am J Ophthalmol 2005;140:679-687.

33. Bansback N, Czoski-Murray C, Carlton J, et al. Determinants of health related quality of life and health state utility in patients with age related macular degeneration: the association of contrast sensitivity and visual acuity. Qual Life Res 2007;16:533-543.

34. Espallargues M, Czoski-Murray CJ, Bansback NJ, et al. The impact of age-related macular degeneration on health status utility values. Invest Ophthalmol Vis Sci 2005;46:4016-4023.

35. Brown GC, Sharma S, Brown MM, Kistler J. Utility values and age-related macular degeneration. Arch Ophthalmol 2000;118:47-51.

36. Brown GC. Vision and quality-of-life. Trans Am ophthalmol soc 1999;97:473-511.

37. Bowker SL, Pohar SL, Johnson JA. A cross-sectional study of health-related quality of life deficits in individuals with comorbid diabetes and cancer. Health and quality of life outcomes 2006;4:17.

38. Jones CA, Pohar SL, Warren S, Turpin KV, Warren KG. The burden of multiple sclerosis: a community health survey. Health and quality of life outcomes 2008;6:1.

39. Argon laser photocoagulation for senile macular degeneration. Results of a randomized clinical trial. Arch Ophthalmol 1982;100:912-918. 
40. Krypton laser photocoagulation for neovascular lesions of age-related macular degeneration. Results of a randomized clinical trial. Macular Photocoagulation Study Group. Arch Ophthalmol 1990;108:816824 .

41. Gelisken F, Voelker M, Schwabe R, et al. Full macular translocation versus photodynamic therapy with verteporfin in the treatment of neovascular age-related macular degeneration: 1-year results of a prospective, controlled, randomised pilot trial (FMT-PDT). Graefes Arch Clin Exp Ophthalmol 2007;245:1085-1095.

42. Laser photocoagulation of subfoveal neovascular lesions of age-related macular degeneration. Updated findings from two clinical trials. Macular Photocoagulation Study Group. Arch Ophthalmol 1993;111:1200-1209.

43. Laser photocoagulation of subfoveal recurrent neovascular lesions in age-related macular degeneration. Results of a randomized clinical trial. Macular Photocoagulation Study Group. Arch Ophthalmol 1991;109:1232-1241.

44. Argon laser photocoagulation for neovascular maculopathy. Five-year results from randomized clinical trials. Macular Photocoagulation Study Group. Arch Ophthalmol 1991;109:1109-1114.

45. Shah AM, Bressler NM, Jampol LM. Does Laser Still Have a Role in the Management of Retinal Vascular and Neovascular Diseases? , Am J Ophthalmol: 2011 Elsevier Inc.

46. Hawkins BS, Bressler NM, Miskala PH, et al. Surgery for subfoveal choroidal neovascularization in agerelated macular degeneration: ophthalmic findings: SST report no. 11. Ophthalmology 2004;111:1967-1980.

47. Kaiser PK. Verteporfin therapy of subfoveal choroidal neovascularization in age-related macular degeneration: 5-year results of two randomized clinical trials with an open-label extension: TAP report no. 8. Graefes Arch Clin Exp Ophthalmol 2006;244:1132-1142.

48. Blumenkranz MS, Bressler NM, Bressler SB, et al. Verteporfin therapy for subfoveal choroidal neovascularization in age-related macular degeneration: three-year results of an open-label extension of 2 randomized clinical trials--TAP Report no. 5. Arch Ophthalmol 2002;120:1307-1314.

49. Bressler NM. Photodynamic therapy of subfoveal choroidal neovascularization in age-related macular degeneration with verteporfin: two-year results of 2 randomized clinical trials-tap report 2. Arch Ophthalmol 2001;119:198-207.

50. Agarwal A, Rhoades WR, Hanout M, et al. Management of neovascular age-related macular degeneration: current state-of-the-art care for optimizing visual outcomes and therapies in development. Clinical ophthalmology (Auckland, NZ) 2015;9:1001-1015.

51. Brechner RJ, Rosenfeld PJ, Babish JD, Caplan S. Pharmacotherapy for neovascular age-related macular degeneration: an analysis of the 100\% 2008 medicare fee-for-service part B claims file. Am J Ophthalmol. United States: Published by Elsevier Inc.; 2010:887-895 e881.

52. Martin DF, Maguire MG, Fine SL, et al. Ranibizumab and bevacizumab for treatment of neovascular age-related macular degeneration: two-year results. Ophthalmology 2012;119:1388-1398.

53. Rosenfeld PJ, Brown DM, Heier JS, et al. Ranibizumab for neovascular age-related macular degeneration. N Engl J Med 2006;355:1419-1431.

54. Brown DM, Michels M, Kaiser PK, Heier JS, Sy JP, Ianchulev T. Ranibizumab versus verteporfin photodynamic therapy for neovascular age-related macular degeneration: Two-year results of the ANCHOR study. Ophthalmology 2009;116:57-65 e55.

55. The CATT research group. Ranibizumab and Bevacizumab for Neovascular Age-Related Macular Degeneration. 2011.

56. Schmidt-Erfurth U, Kaiser PK, Korobelnik JF, et al. Intravitreal aflibercept injection for neovascular age-related macular degeneration: ninety-six-week results of the VIEW studies. Ophthalmology 2014;121:193-201. 
57. Heier JS, Brown DM, Chong V, et al. Intravitreal aflibercept (VEGF trap-eye) in wet age-related macular degeneration. Ophthalmology 2012;119:2537-2548.

58. Abedi F, Wickremasinghe S, Islam AF, Inglis KM, Guymer RH. Anti-VEGF treatment in neovascular age-related macular degeneration: a treat-and-extend protocol over 2 years. Retina 2014;34:1531-1538.

59. Chin-Yee D, Eck T, Fowler S, Hardi A, Apte RS. A systematic review of as needed versus treat and extend ranibizumab or bevacizumab treatment regimens for neovascular age-related macular degeneration. Br J Ophthalmol 2016;100:914-917.

6o. Toalster N, Russell M, Ng P. A 12-month prospective trial of inject and extend regimen for ranibizumab treatment of age-related macular degeneration. Retina 2013;33:1351-1358.

61. Cosgrove T. Value-Based Health Care is Inevitable and That's Good. Harvard Business Review 2013.

62. Porter ME. A strategy for health care reform--toward a value-based system. N Engl J Med 2009;361:109-112.

63. Porter ME. What is value in health care? N Engl J Med 2010;363:2477-2481.

64. Porter ME, Teisberg EO. How physicians can change the future of health care. JAMA 2007;297:1103-1111.

65. Population projections. 1st January population by sex and 5-year age groups http://epp.eurostat. ec.europa.eu/cache/ITY_OFFPUB/KS-SF-11-023/EN/KS-SF-11-023-EN.PDF [accessed 14 July 2011]. Eurostat; 2011 .

66. Owen CG, Jarrar Z, Wormald R, Cook DG, Fletcher AE, Rudnicka AR. The estimated prevalence and incidence of late stage age related macular degeneration in the UK. Br J Ophthalmol 2012;96:752-756.

67. Mitchell J, Wolffsohn JS, Woodcock A, et al. +Psychometric evaluation of the MacDQoL individualised measure of the impact of macular degeneration on quality of life. Health and quality of life outcomes 2005;3:25

68. Feeny D, Furlong W, Torrance GW, et al. Multiattribute and single-attribute utility functions for the health utilities index mark 3 system. Med Care 2002;40:113-128.

69. Brown MM, Brown GC. Update on value-based medicine. Curr Opin Ophthalmol 2013;24:183-189. 

4.
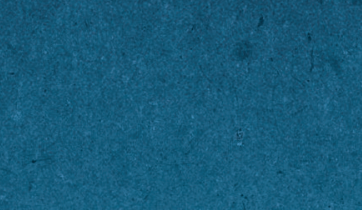

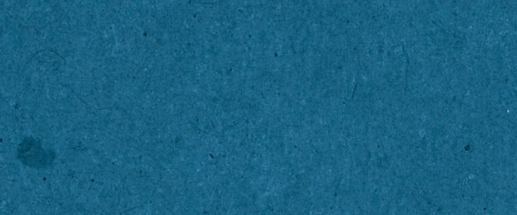
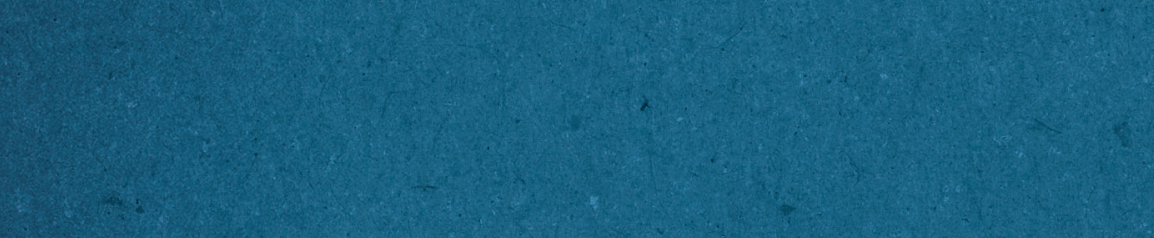

8

$y 3$

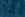




\section{Chapter 2}

Tracing the natural course of visual acuity and quality of life in neovascular age-related macular degeneration: a systematic review and quality of life study

Mari Elshout, Carroll A.B. Webers, Margriet I. van der Reis,

Yvonne de Jong-Hesse, Jan S.A.G. Schouten

BMC Ophthalmology 2017; 17: 120 


\section{Abstract}

Background: Describing the natural course of neovascular age-related macular degeneration (nAMD) is essential in discussing prognosis and treatment options with patients and to support cost-effectiveness studies.

Methods: First, we performed a literature search in PubMed, Embase, and Cochrane. We included randomised clinical trials and prospective observational studies reporting visual acuity (VA) in non-treated patients, 24 studies in total. We integrated VA data using best fit on Lineweaver-Burke plots and modelled with non-linear regression using reciprocal terms. Second, we performed a quality-of-life (QoL) study in nAMD patients. We measured VA with Radner reading charts and QoL with the Health Utilities Index issue 3 (HUI-3) questionnaire in 184 participants. We studied the relation VA-QoL with linear regression. Third, with Monte Carlo simulation, we integrated the VA model from the literature review and the relation VA-QoL from the QoL study.

Results: Visual acuity was 0.4 and 0.07 after 5 years in the better-seeing, and worseseeing eye, respectively. After 4.3 years, VA was $<0.5$ in the better-seeing eye; $<0.3$ after 7 years; 0.05 after 17 years. QoL score decreased from 0.6 to 0.45 after 10 years.

Conclusions: The natural course of nAMD in both eyes needs to be considered when informing patients. Visual acuity in the best eye decreases to below 0.5 in 4.3 years. This affects QoL significantly. 


\section{Background}

Neovascular age-related macular degeneration (nAMD) is a common disorder of the ageing eye. Left untreated, it leads to severe central visual impairment.

"Will I become blind?" is a question patients with nAMD commonly have. Blindness is feared among patients. ${ }^{1}$ Legal blindness of the affected eye is inevitable when nAMD is left untreated. Answering questions regarding the outcome of nAMD requires making predictions. Predicting prognosis is one of the most challenging aspects of clinical medicine, for several reasons. First, a degree of uncertainty exists in results presented in scientific literature. Second, uncertainty is involved when estimating prognosis for the individual patient. Third, nAMD is a bilateral disease. Binocular legal blindness and severe loss of quality of life (QoL) will not occur until the fellow eye becomes significantly affected by nAMD. As QoL greatly depends on visual acuity (VA) in the better-seeing eye, VA in each of both eyes needs to be taken into account when predicting outcomes.

New treatments have become available for nAMD, along with concerns regarding the associated utilisation of resources. In this context, cost-effectiveness studies have been published on the subject of treating nAMD. ${ }^{2}$ In performing a cost-effectiveness study, a good estimate of the natural progression of nAMD is useful for modelling a comparator treatment such as "best supportive care" without an active treatment, or the course of the disease after discontinuation of treatment. ${ }^{3}$ As trials with no-treatment arms are no longer being conducted, estimates of VA and QoL in non-treated eyes have to be based on existing data. Basing assumptions on only one or a few studies may render the results less precise than basing them on results of a systematic review.

In the systematic review section of this study, the question being addressed is what the VA loss is over time in eyes affected with nAMD. Therefore, we selected studies involving patients with nAMD and with the neovascular lesion specified as occult, minimally classic or predominantly classic; not receiving an active intervention; and with VA as an outcome. Study designs could be retrospective studies, prospective clinical studies or observational cohort studies. In the QoL study section of this study, we address the correlation of VA and QoL in order to translate VA results from our review to QoL scores. In this article we describe the long-term natural course of VA and QoL in each eye of patients with nAMD. The results will help the clinician to inform patients about their future VA and to discuss treatment options. Furthermore, the results can be used in costeffectiveness analysis when comparing a proposed treatment to a "no treatment" or "best supportive care" alternative. 


\section{Methods}

\section{Visual acuity: literature search and meta-analysis}

We collected and integrated VA data in a systematic literature review with meta-analysis. We searched for articles in the Databases PubMed; Embase; and the Cochrane databases on Systematic Reviews and Clinical Trials with the last search on 20 January 2015 with no date limits applied. We used the following search terms in the databases: (Macula* OR AMD) AND (follow-up OR longitudinal OR outcome OR randomised OR epidemiologic*) AND (vision OR visual acuity) AND (placebo OR sham OR natural OR no-treatment OR untreated).

One author (ME) was involved in the literature selection process. We included published studies that included non-treated or placebo-treated nAMD patient groups. Studies needed to report separate baseline and follow-up VA outcomes for occult (OC), minimally classic (MC) and/or predominantly classic (PC) lesions. Follow-up period was one year at minimum. Exclusion criteria were: disease studied other than nAMD; publication types such as reviews and conference abstracts; VA not reported as outcome; only treated patients described; the lesion types not being specified; Languages used other than Dutch; English; French; German and Spanish. No additional public protocols are available. We assessed titles and abstracts of the articles returned by the search and excluded articles that were not of relevance. The remaining articles were in- or excluded after reading and assessing the full-text.

Data sought and recorded were neovascular lesion type; baseline VA; follow-up VA values and the corresponding follow-up time points. We performed data extraction on the principal summary measure (VA \& time-point) by recording all VA data from the report with the corresponding time-point of measuring, copying directly from the full-text or tables; and digitally measuring VA and time-point in figures. Risk of bias was not assessed, as we studied only non-treatment arms of included studies.

We integrated VA data using the method of Shah et al.4,5 This is a published method which offers solutions for several concerns in combining data regarding VA loss in nAMD: The difference in average baseline VA among studies; the non-linear progression of VA loss; and the variety of the course of VA between studies. The method is based on the assumption that the difference in baseline VA, between studies and between lesion types, arises from the difference in time of inclusion of patients in the studies. Further, it assumes the progression of VA to be non-linear over time. Rather, it assumes that VA loss follows a reciprocal course, which fits the perception in nAMD that VA loss is more severe immediately after the occurrence of exudative disease, and tends to plateau to an end-stage VA level. 
The method involves the following steps: First, we Integrated VA data on double reciprocal (Lineweaver-Burke) plots. Here, the x-axis represented I/(Months After Enrolment). The y-axis represented 1/(Letters Lost), where Letters Lost $=85$ - Study VA, in Early Treatment Diabetic Retinopathy Study (ETDRS) Letters. We combined the data of all included studies in one plot. We also combined VA data for each lesion type separately on three more plots.

Second, we added a horizontal translation factor, to the initial time values of each individual data set, expressed in months. This changed the horizontal axis from 1 /(Months After Enrolment) to $1 /$ (Months of Exudative Disease) where Months of Exudative Disease = Months After Enrolment + Horizontal Translation Factor. In the previous studies by Shah et al., to determine the optimum horizontal translation factor for each data subset, an arbitrary horizontal translation factor to each data subset was added until the correlation coefficient $r^{2}$ for the overall linear trend line was maximised. This was done in an iterative fashion until there was no further change in $r^{2}$ as additional translations were introduced. Given the number of studies to be included in our study, a manual iterative method seemed neither practical nor precise. We employed a Macro-operated Excel spreadsheet to add arbitrary horizontal translation factors to each subset in an automated iterative fashion. The analysis comprised 1,000,000 iterations in total, from which we selected the set of horizontal translations producing the best linear fit (highest $r^{2}$ ). Consecutively, we could express VA as a function of 'Months of Exudative Disease', with non-linear regression using reciprocal terms. The general formula of such a double reciprocal model is as follows:

$\frac{1}{\text { (Letters lost) }}=a+\frac{b}{t}$

Here, $t=$ Month of Exudative Disease. End-stage VA loss approaches $1 / a$. With higher $a$, endstage VA is better. ${ }^{5}$ With lower $b$, the progression toward end-stage VA loss is more rapid.

\section{Quality-of-life study}

We undertook a cross-sectional study to measure QoL in nAMD patients. We included patients from the University Eye Clinic Maastricht; the Catharina Hospital Eindhoven; the VU University Medical Center in Amsterdam, and ZorgSaam Hospital ZeeuwsVlaanderen, in The Netherlands. The Medical Ethical Committees of all centers approved the design of the study according to Dutch law and the Declaration of Helsinki. Patients were aged at least 50 years and diagnosed with nAMD with choroidal neovascularisation (CNV) on fluorescein angiography between 1992 and 2011. We screened 1948 files; 588 patients were eligible. One hundred and eighty-four patients agreed to participate. Patients gave written informed consent before taking part. 
Two authors (ME and MvdR) visited the patients at home between March 2010 and November 2011. We measured VA using Radner reading cards, ${ }^{6}$ and by assigning counting fingers or seeing hand motions the appropriate value on the Snellen scale. We converted VA results to ETDRS letters equivalents. We measured QoL using the Health Utilities Index issue 3 (HUI-3) questionnaire? We performed linear regression analysis with the HUI-3 score as dependent variable, and VA in the best eye (ETDRS) as independent variable. Further details on the QoL study can be found in the Appendix. Other results of this study have been published previously. ${ }^{8}$

\section{Integrating visual acuity and quality-of-life}

We traced the natural progression of VA in $\mathrm{AMMD}$, with the corresponding deterioration in QoL. We used a Monte Carlo simulation developed for the progression of non-treated and treated eyes with nAMD. 9 The technical structure of the nAMD model was based on an earlier model for studying the progression of glaucoma. ${ }^{10}$ The simulation was developed in Microsoft Excel. In the simulation, virtual patients ('entities') are simulated one by one. The simulation traces the course of VA in both, treated and non-treated, eyes. In the simulation for this study, both eyes remained non-treated. At baseline, both eyes have good VA, and at least one eye is affected. A number of parameters in the model define the longitudinal behaviour of VA and QoL in the simulation. First, VA in affected eyes is defined by the reciprocal terms from the regression analysis on the combined literature analysis described earlier in this paper. Second, the conversion of non-affected eyes to affected eyes is based on a hazard rate, which is calculated using the incidence of nAMD in fellow eyes, based on an earlier review." Third, QoL was calculated using the regression coefficients from the QoL study described earlier in this paper. One thousand entities were simulated and the average loss of VA and QoL in each eye could be traced. Further details on the Monte Carlo simulation and the parameters used can be found in the Appendix. 


\section{Results}

\section{Review: visual acuity}

The literature search returned a total of 1385 articles. All articles were assessed for eligibility. The number of articles excluded based on title and abstract screen was 218. We included 24 articles in the review, totaling 2293 affected, non-treated eyes. Table 1 displays a summary of the studies. Table 1 also lists the horizontal translation factors added to the original data to achieve the best linear fit on the double reciprocal scale. Figure 1 shows the results of the Shah 5 analysis, all included studies combined. Figure 2 shows the results of the analysis separated per lesion type. The Shah analysis produces essentially a non-linear analysis with reciprocal terms $a$ and $b$. These terms are specified in Table 2. End-stage VA loss approaches $1 / a$. With a higher $a$, the end-stage VA is better. With a lower $b$, the progression toward the end-stage VA loss is more rapid. The model defined by the reciprocal terms from analysis that includes all studies and lesion types is used in the Monte Carlo simulation to calculate VA over time. The total loss in VA over 24 months is greatest in PC lesions, least in OC lesions, and intermediate in MC lesions.

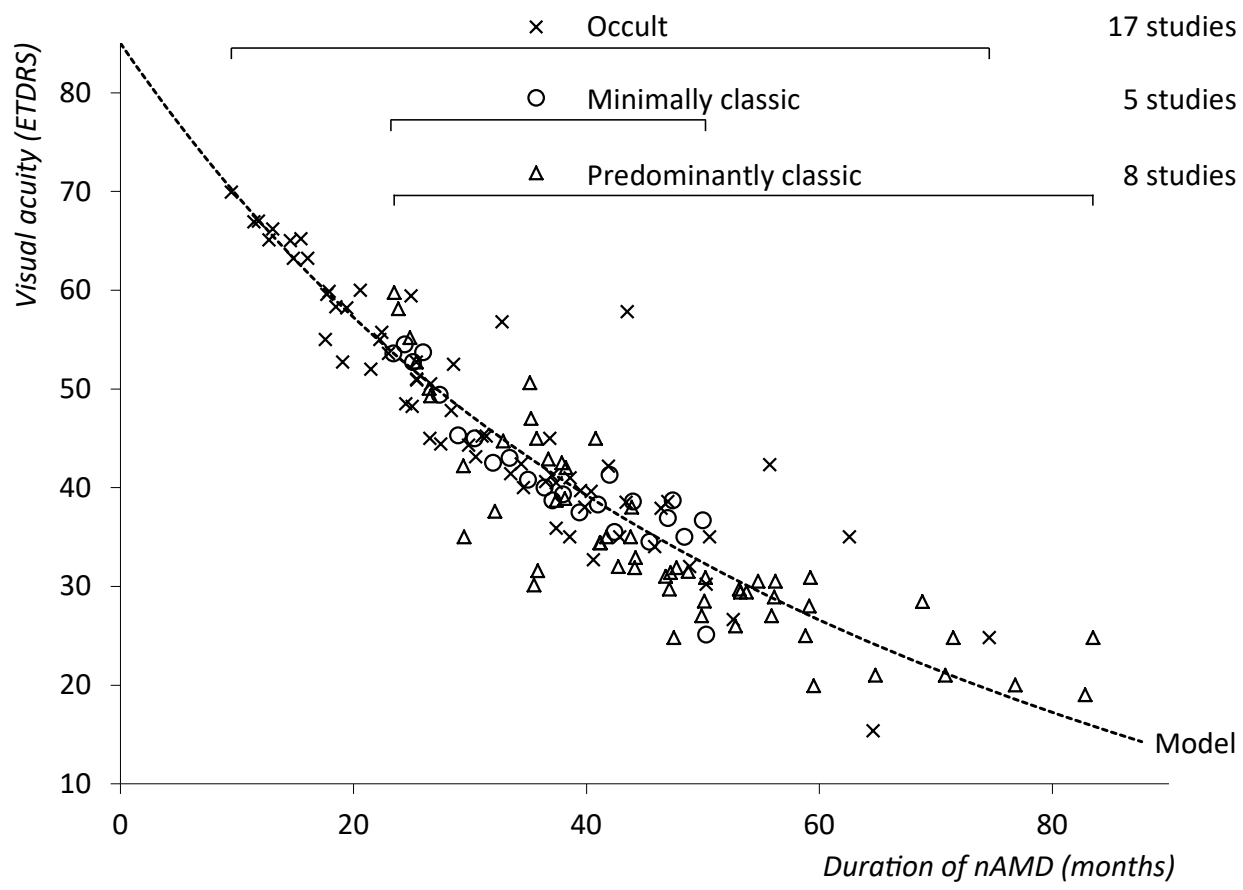

Figure 1. Visual acuity in eyes with non-treated neovascular age-related macular degeneration. Combined analysis. $n A M D$, neovascular age-related macular degeneration. 
Table 1. Studies describing visual acuity loss secondary to neovascular age-related macular degeneration.

\begin{tabular}{|c|c|c|c|c|c|c|}
\hline \multirow[b]{2}{*}{ Study } & \multirow[b]{2}{*}{ Design } & \multirow[b]{2}{*}{$\begin{array}{l}\text { Follow-up } \\
\text { (Months) }\end{array}$} & \multirow[b]{2}{*}{ Eyes } & \multirow{2}{*}{$\begin{array}{c}\text { Baseline } \\
\text { VA } \\
\text { (ETDRS) }\end{array}$} & \multicolumn{2}{|c|}{$\begin{array}{l}\text { Horizontal Translation Factor } \\
\text { (Months) }\end{array}$} \\
\hline & & & & & $\begin{array}{l}\text { Lesion-specific } \\
\text { Analysis }\end{array}$ & $\begin{array}{l}\text { Combined } \\
\text { Analysis }\end{array}$ \\
\hline \multicolumn{7}{|l|}{ Occult lesions } \\
\hline Arnold ${ }^{16}$ & $\mathrm{RCT}$ & 38 & 29 & 60.3 & 15.85 & 17.7 \\
\hline Blinder - TAP \& VIP'17 & $\mathrm{RCT}$ & 24 & 92 & 65.2 & 14.38 & 15.5 \\
\hline Boyer - MARINA ${ }^{18}$ & $\mathrm{RCT}$ & 24 & 151 & 53.6 & 21.63 & 23.0 \\
\hline Bressler $^{19}$ & OCS & 36 & 84 & 55 & 21.52 & 22.3 \\
\hline Bressler - MPS 20 & $\mathrm{RS}$ & 60 & 26 & 65 & 13.31 & 14.6 \\
\hline Cardillo-Piccolino ${ }^{21}$ & OCS & 20 & 63 & 65.1 & 11.70 & 12.8 \\
\hline Eter $^{22}$ & $\mathrm{RCT}$ & 6 & 15 & 58.2 & 18.32 & 19.5 \\
\hline Gragoudas - VISION ${ }^{23}$ & $\mathrm{RCT}$ & 12 & 120 & 52.7 & 24.77 & 25.4 \\
\hline Gustavsson $^{24}$ & $\mathrm{RCT}$ & 12 & 9 & 45 & 40.46 & 36.9 \\
\hline Jaakkola $^{25}$ & $\mathrm{RCT}$ & 36 & 45 & 52.5 & 29.87 & 28.6 \\
\hline Kaiser - VIO ${ }^{26}$ & $\mathrm{RCT}$ & 24 & 120 & 55.7 & 21.26 & 22.4 \\
\hline Kobayashi $^{27}$ & $\mathrm{RCT}$ & 24 & 7 & 59.9 & 16.12 & 17.9 \\
\hline Krebs $^{28}$ & OCs & 3 & 107 & 67 & 10.46 & 11.9 \\
\hline Myint $^{29}$ & $\mathrm{RCT}$ & 12 & 11 & 50.5 & 24.38 & 26.6 \\
\hline $\mathrm{Pece}^{30}$ & OCS & 13.5 & 383 & 66.9 & 10.29 & 11.5 \\
\hline Soubrane $e^{31}$ & RS & 34 & 82 & 69.9 & 8.42 & 9.5 \\
\hline Tholen $^{32}$ & $\mathrm{RCT}$ & 24 & 21 & 66.2 & 11.86 & 13.1 \\
\hline \multicolumn{7}{|c|}{ Minimally classic lesions } \\
\hline Bressler - VIM ${ }^{33}$ & $\mathrm{RCT}$ & 24 & 40 & 54.5 & 5.49 & 24.42 \\
\hline Blinder - TAP \& VIP'7 & $\mathrm{RCT}$ & 24 & 104 & 53.7 & 6.22 & 23.43 \\
\hline Gragoudas - VISION ${ }^{23}$ & $\mathrm{RCT}$ & 12 & 102 & 52.7 & 6.41 & 25.10 \\
\hline Schneider ${ }^{34}$ & $\mathrm{RS}$ & 8.3 & 4 & 41.3 & 20.74 & 41.99 \\
\hline Boyer - MARINA $^{18}$ & $\mathrm{RCT}$ & 24 & 87 & 53.6 & 6.04 & 23.43 \\
\hline \multicolumn{7}{|c|}{ Predominantly classic lesions } \\
\hline D'Amico - $\mathrm{AA}^{35,36}$ & $\mathrm{RCT}$ & 24 & 30 & 47 & 6.35 & 35.24 \\
\hline Blinder - TAP \& VIP ${ }^{17}$ & $\mathrm{RCT}$ & 24 & 83 & 50.6 & 5.60 & 35.14 \\
\hline Bressler - MPS ${ }^{20}$ & RS & 60 & 119 & 59.7 & 3.15 & 23.51 \\
\hline Gragoudas - VISION ${ }^{23}$ & $\mathrm{RCT}$ & 12 & 76 & 52.7 & 4.59 & 25.40 \\
\hline MPS 37 & RS & 42 & 184 & 45 & 7.89 & 40.82 \\
\hline Mandai ${ }^{38}$ & $\mathrm{RCT}$ & 36 & 4 & 44.7 & 7.53 & 32.85 \\
\hline Valmaggia39 & $\mathrm{RCT}$ & 18 & 29 & 42.5 & 7.68 & 37.90 \\
\hline Behrendt ${ }^{40}$ & $\mathrm{RCT}$ & 12 & 66 & 58 & 3.17 & 23.85 \\
\hline
\end{tabular}

ETDRS, Early Treatment Diabetic Retinopathy Study, OCS, observational case series, RCT randomised controlled trial, $R S$ retrospective study, VA visual acuity. 


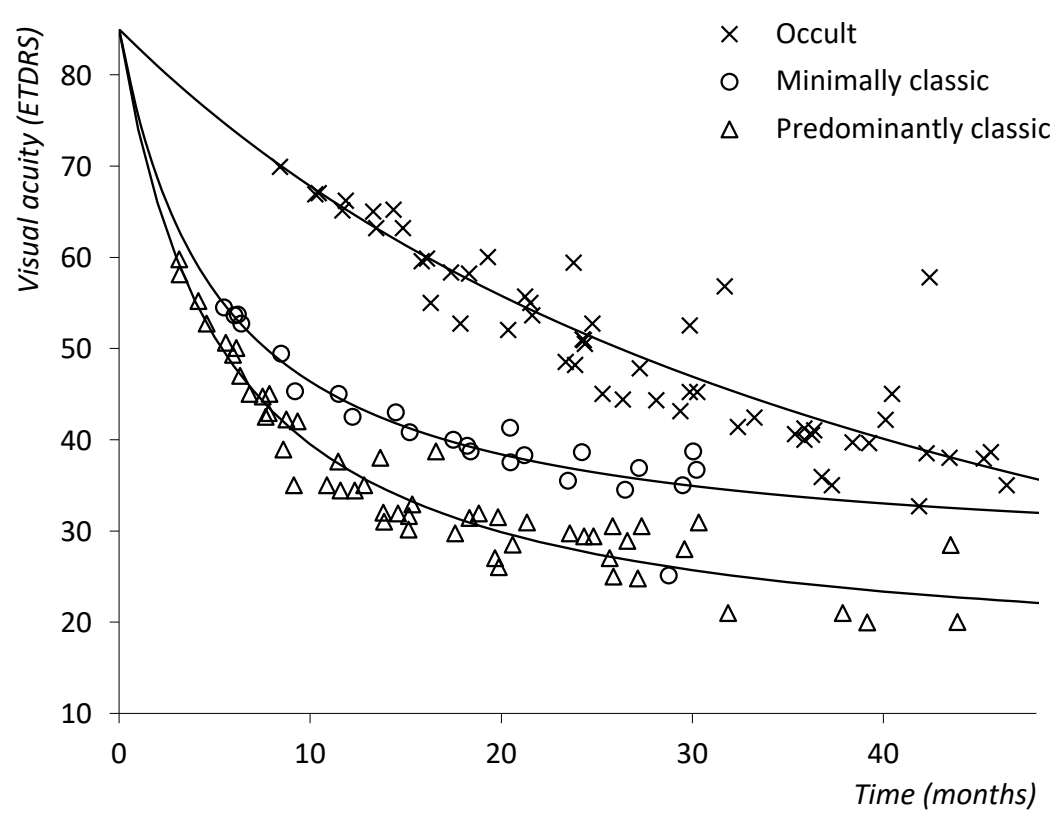

Figure 2. Visual acuity in eyes with neovascular age-related macular degeneration. Separate analysis, per lesion type.

\section{Quality of life}

The QoL study included 184 patients. Average age was 81 years, $52 \%$ were female. Participants had an average disease duration of 5.8 years. Visual acuity was 49 letters in the better-seeing eye; 3.5 letters in the worse-seeing eye. Average HUI-3 score was 0.46. Univariate regression analysis showed the highest correlation $\left(r^{2}\right)$ of QoL with VA in the better-seeing eye (0.20) and VA in both eyes (0.21) than with VA in the worse eye (o.08). Gender and age were not statistically significant predictors of QoL. The presence of comorbidities had a statistically significant impact on QoL, but did not change the correlation parameters of VA in the better-seeing eye in multivariate regression analysis. Because of sufficient correlation with QoL, we used the linear regression coefficient of VA in the better-seeing eye as a factor to calculate QoL in further analysis described below. The linear regression coefficient was 0.004 ( $p 0.000$ ), with a constant of 0.270 . This implies a 0.004 decrease in HUI-3 QoL score per letter of VA lost. Further details of the QoL study can be found in the Appendix. 
Table 2. Parameters to calculate expected visual acuity loss in neovascular age-related macular degeneration.

\begin{tabular}{lccc}
\hline & \multicolumn{2}{c}{ Parameter } & \\
\cline { 2 - 3 } Lesion type & $a$ & $b$ & $\mathrm{R}^{2}$ \\
\hline Combined model & 0.567 & 0.0077 & 0.91 \\
Occult & 0.478 & 0.010 & 0.90 \\
Minimally classic & 0.089 & 0.017 & 0.95 \\
Predominantly classic & 0.077 & 0.014 & 0.96 \\
\hline
\end{tabular}

l/letters lost $=a+b /$ months

\section{Integrating visual acuity and quality-of-life}

The Monte Carlo simulation traced the course of VA over time in both non-treated eyes of nAMD patients, see Figure 3. The first affected eyes show average VA loss that reflects the double reciprocal model of the literature analysis, shown in Figure 1. The betterseeing eyes (or 'fellow eyes') are mostly unaffected at baseline. A growing proportion of these fellow eyes become affected over time. Therefore, Figure 3 shows that the betterseeing eyes lose VA more gradually than do the primarily affected eyes. In the Figure, visual acuity can be traced at selected time-points. After five years, VA is 26.7 letters (0.0) Snellen) in the worse-seeing eye, and 66.8 letters ( 0.43 Snellen) in the better-seeing eye After ten years, VA is 4.5 letters ( 0.02 Snellen) in the worse-seeing eye, and 49.8 letters ( 0.2 Snellen) in the better-seeing eye. Also, the time until certain VA thresholds are reached can be traced. In the better-seeing eye, A Snellen VA of $<0.5$ (70 ETDRS letters) is reached after an average of 4.3 years. VA $<0.3$ (6o letters) is reached after 6.8 years; $<0.05$ (20 letters) after 17.3 years.

The simulation also traced QoL over time. Quality-of-life was calculated from betterseeing eye VA using the linear parameters from the QoL study. The QoL values over time are displayed in Figure 4. Average VA in the better-seeing eye decreases over time. Hence, QoL also decreases over time. 


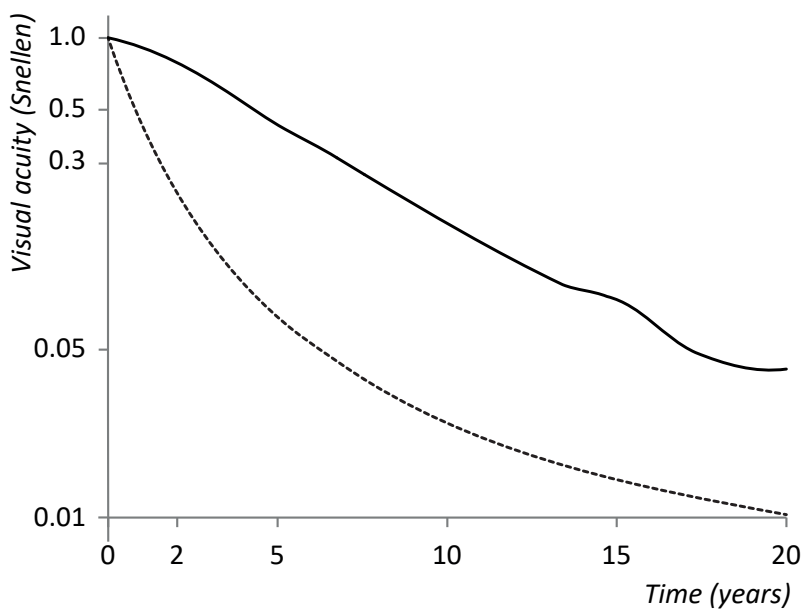

\begin{tabular}{llcc} 
& & $\begin{array}{c}\text { Initially affected } \\
\text { eye }\end{array}$ & $\begin{array}{c}\text { Better-seeing } \\
\text { eye }\end{array}$ \\
\hline $\begin{array}{l}\text { VA } \\
\text { (Snellen) }\end{array}$ & after 2 years & 0.23 & 0.80 \\
after 5 years & 0.07 & 0.43 \\
\hline Duration & to VA $<0.5$ & 1.3 & 4.3 \\
(years) & to VA $<0.3$ & 2.0 & 6.8 \\
& to VA $<0.05$ & 6.7 & 17.3 \\
\hline
\end{tabular}

Figure 3. Visual acuity development and benchmarks in neovascular age related macular degeneration. $V A$, visual acuity.

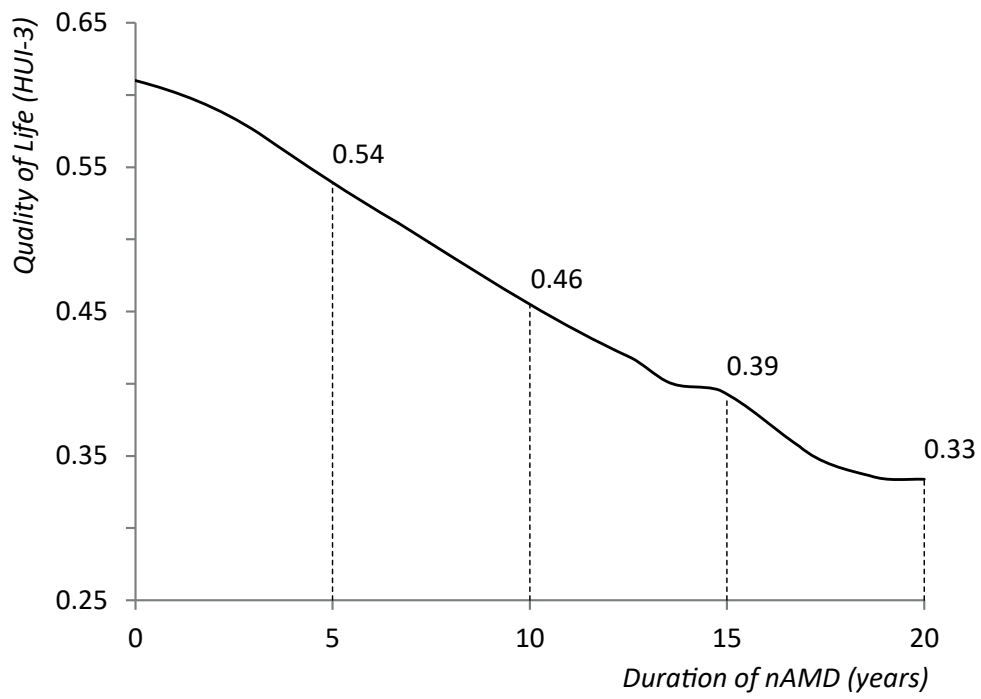

Figure 4. Quality-of-life over time in non-treated neovascular age-related macular degeneration. 


\section{Discussion}

We integrated VA data from non-treated nAMD patients from multiple studies. The included studies differed by the type of CNV lesion studied; the baseline VA of the patients; the duration of follow-up, and the timing of follow-up VA measurements. A fitting solution to these issues was demonstrated in previous studies using double reciprocal plots and adding horizontal translation factors. ${ }^{4.5}$ The review is limited by the fact that study size could not be accounted for in the analysis, which is inherent to the method used. The combined analysis shows that occult lesions are associated with better VA, and are seen more often earlier in the disease course. Later, classic lesions may be seen, with generally worse VA. Initially, VA loss is about 1.25 ETDRS letters per month.

The integrated VA results from the review were combined with QoL data from a crosssectional QoL study into a Monte Carlo analysis that longitudinally traced VA and QoL in typical patients. Rates of function loss and the corresponding changes in QoL have been studied previously in other eye diseases. For example, a study by Medeiros et al. evaluated the rates of visual field loss and longitudinal changes in QoL in glaucoma patients. ${ }^{12}$ In this study, patients received QoL measurements annually and perimetry at six-month intervals, and a significant correlation between QoL score and binocular perimetry sensitivity was demonstrated. Recruitment and follow-up of patients is timeand capital-intensive, and sometimes, when studying the natural course of a disease, may encounter ethical barriers. Computational modelling can solve such issues. Using modelling to translate cross-sectional data into longitudinal outcomes, as in our study, has also been performed previously. Our model was based on a disease-progression model developed and validated for the progression of glaucoma by Van Gestel et al. ${ }^{10}$ Similar to our study, QoL on the HUI-3 scale was calculated using the linear coefficients from regression analysis. In the glaucoma model, QoL was based in part on mean deviation on the visual fields, using a cross-sectional QoL study in glaucoma patients. In our study, QoL was calculated using the coefficients from our cross-sectional QoL study in nAMD patients. The glaucoma model also simulated conversion from ocular hypertension to glaucoma, using a hazard rate to generate a distribution of time-to-conversion. In a technically similar approach, our model generated a distribution of time to the development of nAMD in a non-affected eye using a hazard rate from a previous review." The glaucoma model used the rate of mean deviation from a glaucoma population, while we used the rate of loss of VA from the review presented here.

With untreated nAMD, VA decreases, and QoL declines significantly. Visual acuity loss impacts QoL in part through a negative impact on activities of daily living, and the impact on these activities is considerable. As an example, one of the more highly valued activities 
by individuals is driving. Driving is allowed with VA of $\geq 0.5$ Snellen in a least one eye. Left untreated, nAMD causes a VA loss to below 0.5 within 5 years in the better-seeing eye. Patients may lose about $1 / 4$ of their initial QoL in the first 10 years. Quality of life scores for patients with nAMD are comparable to or worse than with several other chronic and/ or debilitating diseases, and may decline further over years. Patients with nAMD progress through QoL scores lower than those for example in persons with diabetes (HUI-3 score 0.78), colorectal cancer (HUI-3 score 0.78), ${ }^{13}$ or multiple sclerosis (HUI-3 score 0.64). ${ }^{14}$ In this perspective, with effective treatments being available, the clinician should take the highest effort to prevent VA loss in nAMD patients.

The results of this study can be used to inform patients in a more accurate and understandable way about their future sight and visual impairment. Researchers and clinicians can use these results when seeking to estimate the natural progression of nAMD. The disease has a great impact on an individual as well as on a societal level. This study can be used to show how large this impact is or how large it can be. There is increasing discussion regarding the benefits and costs of nAMD and its treatments. Model-based cost-effectiveness studies support this discussion with evidence. ${ }^{15}$ In many such studies, a 'no-treatment' strategy is used as a comparator strategy. As such, information on natural progression of nAMD has again become highly relevant. The formula and parameters provided can be readily implemented in model-based cost-effectiveness studies.

When explaining prognosis to nAMD patients, it is important to describe the expected progression for both eyes. Especially important is the fellow, and mostly better-seeing, eye. If VA decreases here, binocular VA and QoL are greatly affected. The rate of VA loss in affected eyes is well described in multiple studies." Less is published about VA in the fellow eye or about binocular VA. We know that initially, the fellow eye is mostly unaffected. When nAMD occurs, VA decreases. Our simulation incorporates the incidence of $\mathrm{nAMD}$ and the corresponding rate of VA loss in fellow eyes, and predicts outcomes for both eyes.

Will a patient become blind? The World Health Organisation (WHO) provides a definition of blindness as Snellen VA being <0.05. Assuming a non-treated 75-year patient with a life expectancy of 11 years, he or she is unlikely to become completely blind. However, it is of paramount importance to consider the serious impact on QoL. With the availability of current anti-vascular endothelial growth factor (anti-VEGF) treatment, this can be presented as a worst-case scenario, e.g. when treatment fails or is not accepted. When informing patients, the benefit of a treatment can be illustrated by comparing treatment outcomes to a hypothetical scenario in which no treatment would be given. 


\section{Conclusions}

In conclusion, VA data of the natural course of nAMD from multiple studies were integrated. Visual acuity loss was translated with a 2-eye model into a longitudinal trace of QoL. This provides a comprehensive model of the disease in both eyes, with the patients' corresponding general well-being, which can be used to support treatment decisions, and to inform patients about their prognosis. Left untreated, the VA will drop to below 0.5 Snellen within 4.3 years.

\section{Acknowledgements}

The authors thank the Ophthalmology departments of the Catharina Hospital Eindhoven, ZorgSaam Hospital Zeeuws-Vlaanderen, and the VU medical center Amsterdam in The Netherlands, for providing access to their patient database and patient files. 


\section{References}

1. Williams RA, Brody BL, Thomas RG, Kaplan RM, Brown SI. The psychosocial impact of macular degeneration. Arch Ophthalmol 1998;116:514-520.

2. Schmier JK, Hulme-Lowe CK. Cost-Effectiveness Models in Age-Related Macular Degeneration: Issues and Challenges. Pharmacoeconomics 2016;34:259-272.

3. Ghosh W, Wickstead R, Claxton L, et al. The Cost-Effectiveness of Ranibizumab Treat and Extend Regimen Versus Aflibercept in the UK. Advances in therapy 2016;33:1660-1676.

4. Shah AR, Del Priore LV. Natural history of predominantly classic, minimally classic, and occult subgroups in exudative age-related macular degeneration. Ophthalmology 2009;116:1901-1907.

5. Shah AR, Del Priore LV. Progressive visual loss in subfoveal exudation in age-related macular degeneration: a meta-analysis using Lineweaver-Burke plots. Am J Ophthalmol 2007;143:83-89.

6. Radner W, Willinger U, Obermayer W, Mudrich C, Velikay-Parel M, Eisenwort B. [A new reading chart for simultaneous determination of reading vision and reading speed]. Klin Monbl Augenheilkd 1998;213:174-181.

7. Feeny D, Furlong W, Torrance GW, et al. Multiattribute and single-attribute utility functions for the health utilities index mark 3 system. Med Care 2002;40:113-128.

8. Elshout M, van der Reis MI, de Jong-Hesse Y, Webers CA, Schouten JS. Distinguishing between Better and Worse Visual Acuity by Studying the Correlation with Quality of Life in Neovascular Age-Related Macular Degeneration. Ophthalmology 2016;123:2408-2412.

9. Elshout M, van der Reis MI, Webers CA, Schouten JS. The cost-utility of aflibercept for the treatment of age-related macular degeneration compared to bevacizumab and ranibizumab and the influence of model parameters. Graefes Arch Clin Exp Ophthalmol 2014.

10. Van Gestel A, Severens JL, Webers CA, Beckers HJ, Jansonius NM, Schouten JS. Modeling complex treatment strategies: construction and validation of a discrete event simulation model for glaucoma. Value Health 2010;13:358-367.

11. Wong TY, Chakravarthy U, Klein R, et al. The natural history and prognosis of neovascular age-related macular degeneration: a systematic review of the literature and meta-analysis. Ophthalmology 2008;115:116-126.

12. Medeiros FA, Gracitelli CP, Boer ER, Weinreb RN, Zangwill LM, Rosen PN. Longitudinal changes in quality of life and rates of progressive visual field loss in glaucoma patients. Ophthalmology 2015;122:293-301.

13. Bowker SL, Pohar SL, Johnson JA. A cross-sectional study of health-related quality of life deficits in individuals with comorbid diabetes and cancer. Health and quality of life outcomes 2006;4:17.

14. Jones CA, Pohar SL, Warren S, Turpin KV, Warren KG. The burden of multiple sclerosis: a community health survey. Health and quality of life outcomes 2008;6:1.

15. Schmier JK, Hulme-Lowe CK. Cost-Effectiveness Models in Age-Related Macular Degeneration: Issues and Challenges. Pharmacoeconomics 2015.

16. Arnold J, Barbazetto I, Birngruber R, et al. Photodynamic therapy of subfoveal choroidal neovascularization in age-related macular degeneration with verteporfin: Two-year results of 2 randomized clinical trials - TAP report 2. Archives of Ophthalmology 2001;119:198-207.

17. Blinder KJ, Bradley S, Bressler NM, et al. Effect of lesion size, visual acuity, and lesion composition on visual acuity change with and without verteporfin therapy for choroidal neovascularization secondary to age-related macular degeneration: TAP and VIP report no. 1. Am J Ophthalmol 2003;136:407-418. 
18. Boyer DS, Antoszyk AN, Awh CC, Bhisitkul RB, Shapiro H, Acharya NR. Subgroup analysis of the MARINA study of ranibizumab in neovascular age-related macular degeneration. Ophthalmology 2007;114:246-252.

19. Bressler NM, Frost LA, Bressler SB, Murphy RP, Fine SL. Natural course of poorly defined choroidal neovascularization associated with macular degeneration. Arch Ophthalmol 1988;106:1537-1542.

20. Bressler. Occult choroidal neovascularization. Influence on visual outcome in patients with age-related macular degeneration. Macular Photocoagulation Study Group. Arch Ophthalmol 1996;114:400-412.

21. Cardillo-Piccolino F, Ghiglione D, Borgia L, Zingirian M. [New occult choroidal vessels in age-related macular degeneration]. J Fr Ophtalmol 1989;12:869-876.

22. Eter N, Schuller H, Spitznas M. Radiotherapy for age-related macular degeneration: is there a benefit for classic CNV? Int Ophthalmol 2001;24:13-19.

23. Gragoudas ES, Adamis AP, Cunningham ET, Jr., Feinsod M, Guyer DR. Pegaptanib for neovascular age-related macular degeneration. N Engl J Med 2004;351:2805-2816.

24. Gustavsson C, Agardh E. Transpupillary thermotherapy for occult subfoveal choroidal neovascularization: a 1-year, prospective randomized pilot study. Acta Ophthalmol Scand 2005;83:148153 .

25. Jaakkola A, Heikkonen J, Tommila P, Laatikainen L, Immonen I. Strontium plaque brachytherapy for exudative age-related macular degeneration: three-year results of a randomized study. Ophthalmology 2005;112:567-573.

26. Kaiser PK. Verteporfin PDT for subfoveal occult CNV in AMD: two-year results of a randomized trial. Curr Med Res Opin 2009;25:1853-1860.

27. Kobayashi H, Kobayashi K. Age-related macular degeneration: long-term results of radiotherapy for subfoveal neovascular membranes. Am J Ophthalmol 2000;130:617-635

28. Krebs I, Stolba U, Glittenberg C, Seyeddain O, Benesch T, Binder S. Prognosis of untreated occult choroidal neovascularization. Graefes Arch Clin Exp Ophthalmol 2007;245:376-384.

29. Myint K, Armbrecht AM, Mon S, Dhillon B. Transpupillary thermotherapy for the treatment of occult CNV in age-related macular degeneration: a prospective randomized controlled pilot study. Acta Ophthalmol Scand 2006;84:328-332.

30. Pece A, Bolognesi G, Introini U, Pacelli G, Calori G, Brancato R. Indocyanine green angiography of welldefined plaque choroidal neovascularization in age-related macular degeneration. Arch Ophthalmol 2000;118:630-634.

31. Soubrane G, Coscas G, Francais C, Koenig F. Occult subretinal new vessels in age-related macular degeneration. Natural History and early laser treatment. Ophthalmology 1990;97:649-657.

32. Tholen AM, Meister A, Bernasconi PP, Messmer EP. [Radiotherapy for choroidal neovascularization (CNV) in age-related macular degeneration (AMD)]. Klin Monbl Augenheilkd 2000;216:112-115.

33. Azab M, Boyer DS, Bressler NM, et al. Verteporfin therapy of subfoveal minimally classic choroidal neovascularization in age-related macular degeneration: 2-year results of a randomized clinical trial. Arch Ophthalmol 2005;123:448-457.

34. Schneider U, Gelisken F, Inhoffen W. Natural course of occult choroidal neovascularization in agerelated macular degeneration: development of classic lesions in fluorescein angiography. Acta Ophthalmol Scand 2005;83:141-147.

35. D'Amico DJ, Goldberg MF, Hudson H, et al. Anecortave acetate as monotherapy for treatment of subfoveal neovascularization in age-related macular degeneration: twelve-month clinical outcomes. Ophthalmology 2003;110:2372-2383; discussin 2384-2375.

36. Russell SR, Hudson HL, Jerdan JA. Anecortave acetate for the treatment of exudative age-related macular degeneration--a review of clinical outcomes. Surv Ophthalmol 2007;52 Suppl 1:S79-90. 
37. Laser photocoagulation of subfoveal recurrent neovascular lesions in age-related macular degeneration. Results of a randomized clinical trial. Macular Photocoagulation Study Group. Arch Ophthalmol 1991;109:1232-1241.

38. Mandai M, Takahashi M, Miyamoto H, et al. Long-term outcome after radiation therapy for subfoveal choroidal neovascularization associated with age-related macular degeneration. Japanese journal of ophthalmology 2000;44:530-537.

39. Valmaggia C, Ries G, Ballinari P. Radiotherapy for subfoveal choroidal neovascularization in agerelated macular degeneration: a randomized clinical trial. Am J Ophthalmol 2002;133:521-529.

40. Behrendt C. Visual acuity and its decrease in classic neovascular age-related macular degeneration. Ophthalmic Epidemiol 2004;11:359-367. 
4.
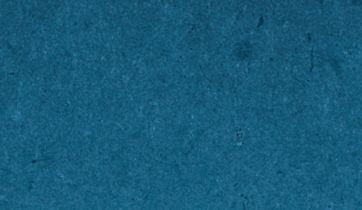

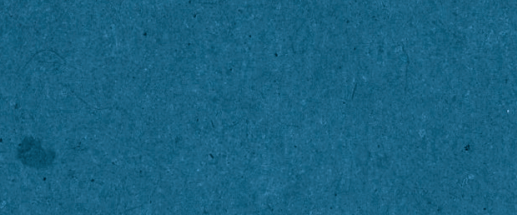
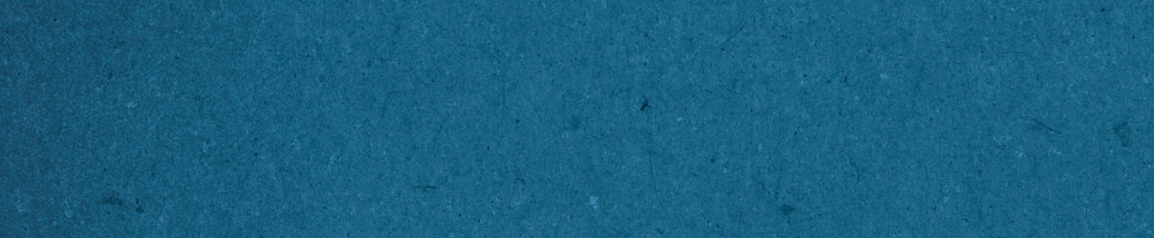

8

$y 3$

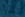




\section{Chapter 2 Appendix}

Further details of methodology of the quality-of-life study and the Monte Carlo simulation 


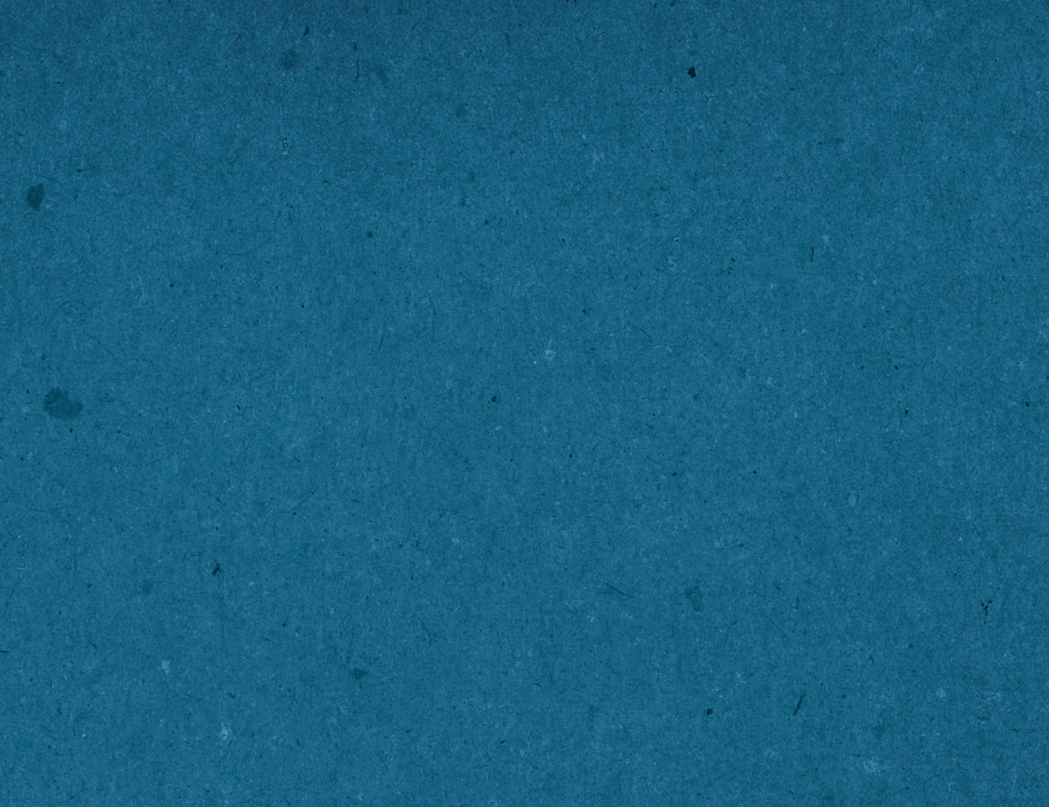




\section{Introduction}

This document is an Appendix to the manuscript "Tracing the natural course of visual acuity and quality of life in neovascular age-related macular degeneration: a systematic review and quality of life study." In the manuscript, the natural course of neovascular age-related macular degeneration (nAMD) is described. The study comprises three main analyses: (1) a literature review and meta-analysis of the longitudinal course of visual acuity; (2) a study of quality of life (QOL) in patients with nAMD; and (3) combining results of the review and QoL study into a Monte Carlo simulation to analyse the longitudinal course progression of visual acuity and QoL over time. As the manuscript comprises multiple sections of specialised analytics, the reader may need further specifications on the methods and analysis that underpin the analyses. This appendix provides further details on the methodology of the QoL study and Monte Carlo simulation. 


\section{The quality-of-life study}

\section{Design and participants}

The quality of life (QoL) study was a cross-sectional, observational study. The study enrolled patients from the University Eye Clinic Maastricht; the Catharina Hospital Eindhoven; ZorgSaam Hospital Zeeuws-Vlaanderen, and the VU University Medical Center in Amsterdam, in The Netherlands. Inclusion criteria were: age of 50 years or more, and a diagnosis with neovascular age-related macular degeneration (nAMD) with choroidal neovascularisation on fluorescein angiography between 1992 and 2011. The number of files screened was 1944. The number of eligible patients was 588. One-hundred and eighty-four patients agreed to participate in the study. Between those patients who participated and those who did not participate, there were no differences in age, gender or geographic location. Questionnaire completion rates exceeded 95\% for the HUI-3 measure. Interviews took place between March 2010 and November 2011.

\section{Data Collection}

Visual acuity (VA) and QoL were measured at the participants' homes. Visual acuity was measured using Radner Reading charts with separate charts for each eye separately and both eyes simultaneously., ${ }^{1,2}$ Counting fingers and seeing hand motions were scored the appropriate values on the decimal visual acuity scale. LogRAD and decimal visual acuity were converted to ETDRS visual acuity equivalents to allow for linear regression analysis. The Health Utilities Index Mark III (HUI-3) was used to measure generic quality of life. The HUI-3 is a utility scale, a questionnaire used to assess the participants' quality of life. Other quality-of-life scales were also applied. The nature of any comorbidities was recorded and scored as on the Charlson Comorbidity Index ${ }^{3}$ (CCI), in order to capture the number and the severity of comorbidities in a single metric ranging from o to 6.

\section{Data analysis and results}

Descriptive analysis was used to summarise the characteristics of the participants, shown in Table 1. Multiple and single linear regression analysis was used to study the relation between VA and QoL. There was a linear trend of better QoL with better VA (Table 2 and Table 3). The highest percentage of explained variability in the single regression analysis $\left(\mathrm{R}^{2}\right)$ was observed for better-seeing eye VA (20\%) and binocular VA (21\%), compared to worse-eye VA (8.6\%).

In a multiple regression model (Table 3), the relationship of both binocular VA and comorbidity with utility reached high statistical significance. Variables 'age' and 'living situation' did not reach significance at the 5\% level. Gender; the interaction of visual acuity with comorbidity; and time since diagnosis were not statistically significant predictors of the HUI-3 score in a multiple regression model. 
Table 1. Characteristics of participants with neovascular age-related macular degeneration.

\begin{tabular}{|c|c|c|}
\hline \multirow{3}{*}{ Age, years } & \multicolumn{2}{|c|}{$N=184$} \\
\hline & \multicolumn{2}{|c|}{ Mean (SD) } \\
\hline & 81.2 & $(7.1)$ \\
\hline Female, \% & 52.2 & \\
\hline Time since diagnosis, years & 5.8 & $(3.4)$ \\
\hline Charlson Comorbidities Index & 0.51 & $(0.9)$ \\
\hline \multicolumn{3}{|l|}{ Better-seeing eye VA } \\
\hline ETDRS & 49 & (32) \\
\hline Snellen & 0.19 & \\
\hline \multicolumn{3}{|l|}{ Fellow eye VA } \\
\hline ETDRS & 3.5 & $(36.5)$ \\
\hline Snellen & 0.023 & \\
\hline \multicolumn{3}{|l|}{ Binocular VA } \\
\hline ETDRS & 46 & (32) \\
\hline Snellen & 0.17 & \\
\hline HUI-3 score & 0.45 & $(0.31)$ \\
\hline
\end{tabular}

ETDRS, Early Treatment Diabetic Retinopathy Study; HUI-3, Health Utilities Index Mark 3; SD, standard deviation; $V A$, visual acuity.

Table 2. Results of single regression analysis for patients with nAMD. Dependent variable: HUI-3 score.

\begin{tabular}{lcccc}
\hline Variable & $\beta$ & SE & Standardised $\beta$ & $P$ \\
\hline $\mathrm{B}_{0}$ (constant) & 0.270 & 0.035 & & 0.000 \\
Best eye VA & 0.004 & 0.001 & 0.445 & 0.000 \\
\hline$R^{2}=0.198$ & & & & \\
$S E$, standard error; $V A$, visual acuity. & & &
\end{tabular}

Table 3. Results of multiple regression analysis for patients with nAMD. Dependent variable: HUI-3 score.

\begin{tabular}{lcccc}
\hline Variable & $\beta$ & SE & Standardised $\beta$ & $P$ \\
\hline B $_{0}$ (constant) & 0.666 & 0.270 & & 0.014 \\
Best eye VA & 0.004 & 0.001 & 0.429 & 0.000 \\
Comorbidity (CCI) & -0.104 & 0.020 & -0.321 & 0.000 \\
Age & -0.006 & 0.003 & -0.129 & 0.057 \\
Living alone (1) / together (2) & 0.076 & 0.040 & 0.123 & 0.057 \\
\hline
\end{tabular}

$R^{2}=0.352$

CCI, Charlson Comorbidity Index; 3 SE, standard error; VA, visual acuity. 


\section{The Monte Carlo simulation}

The computerised model used to trace the natural course of nAMD is a patient-level, discrete-event, Monte Carlo type simulation. Patient-level Monte Carlo means that virtual patients (called 'entities') are simulated one by one. At baseline, or $t=0$ in the simulation, the necessary characteristics of the individual entity, such as visual acuity, but also the timing of certain key events, are defined from a set of distributions. Outcomes of each entity are recorded so that results of a population of entities can be interpreted with appropriate statistics. Discrete-event means that the course of time and the recalculation of entities' parameters model are based on events. Examples of such events are treatments, medical visits, and the end of the time-frame. At every event, a set of characteristics of the entity are re-calculated or re-defined, such as disease state; visual acuity; and the time to the next event.

Technically, the model is a Macro-operated spreadsheet developed in Microsoft Excel. For a single entity, the Excel sheet calculates the complete sequencing and timing of all events and all outcomes at each event, until a pre-set time-horizon is reached. The Macro program instructs the Excel sheet to record all outputs and repeat the calculations for the next entity, until a population of entities is complete. The virtual population comprised 1,000 entities: Averages and standard deviations of VA and QoL outcomes stabilised at around 500 to 600 entities.

The model has been employed previously to calculate the cost-effectiveness of treatments of macular degeneration. ${ }^{4}$ The model was based on a previous model simulating the disease progression of glaucoma. ${ }^{5}$ For the current purpose of tracing the natural course of neovascular age-related macular degeneration, the model could be simplified. In the current model, a limited set of parameters eventually define the outcome of interest: the average course of VA and QoL over time in two eyes. These parameters and their distributions are described below in a point-by-point fashion.

\section{Baseline visual acuity}

Baseline visual acuity of each eye of an entity at $t=0$, affected or not, was assumed to be 85 ETDRS letters, or 1.o Snellen visual acuity.

\section{Proportion of eyes affected at baseline}

An entity had at least one eye affected at baseline: the initially affected eye. The fellow eye could either be affected or not. Each entity had a 5.4\% risk of having the fellow eye affected at baseline. This percentage was based on the clinical data from the 184 participants in the cross-sectional study, of which $5.4 \%$ had bilateral disease at presentation. We found 
no other evidence in literature defining the proportion of nAMD patients at presentation with bilateral active disease.

\section{Course of visual acuity in affected eyes}

The course of visual acuity in affected eyes was defined by the parameters as calculated by the current literature meta-analysis:

$$
\mathrm{VA}_{t}=\mathrm{VA}_{0}-\frac{1}{a+\frac{1}{b}}
$$

\footnotetext{
Where

$\mathrm{VA}_{t}=$ visual acuity at time-point $t$;

$\mathrm{VA}_{\mathrm{o}}=$ visual acuity at baseline (85 ETDRS Letters);

$a \quad=$ parameter a from meta-analysis $=(0.567) ;$

$b \quad=$ parameter $\mathrm{b}$ from meta-analysis $=(0.0077) ;$

$t \quad=$ time (months).
}

One of the characteristics of a discrete-event simulation is that any defined outcome is not continuously updated, but only when an event takes place. This is not an issue as long as there are frequent events and/or the trend of the outcome is linear. As no treatments or other events such as follow-up visits were active in the current simplified model, and the course of visual acuity was not linear, artificial monthly 'update' events were built into the model. This ensured that every month in the simulation, visual acuity and quality of life were updated and traced with precision.

\section{Course of visual acuity in non-affected eyes}

Visual acuity in non-affected eyes was assumed to remain unchanged over time.

\section{Conversion of non-affected to affected eyes}

Non-affected fellow eyes could progress to active nAMD. In this a discrete-event simulation, this conversion was handled as an event. The timing of this event was defined for the entity at $t=0$. Using evidence from the literature, we set the simulation to draw a specific time to conversion for each entity, using the hazard rate. 
For calculating a hazard rate, we used the survival function:

$P=1-S=1-e^{-h \cdot t}$

Where

$P \quad=$ cumulative incidence of $\mathrm{nAMD}$ in the fellow eye;

$S=$ conversion free survival;

$t$ = time (months);

$h=$ hazard rate .

Thus, the hazard rate $h$, which is an instantaneous incidence rate, is defined as:

$$
\frac{\ln (1-P)}{t}=h
$$

An estimate of $P$ was extracted from a natural history review by Wong et al. ${ }^{6}$ We selected this study as it provides the estimate of incidence in the fellow eye based on the largest base of studies. This review aggregated the results of five studies with 541 patients and found a cumulative incidence of nAMD in the fellow eye $P$ of 0.212 , at $t=36$ months.

Assuming that the time-to-event takes on an exponential distribution,? the time-toconversion for the individual entity $t_{i}$ can be defined as:

$t_{i}=\frac{-\ln [1-\alpha]}{h}$

Where $a$ is drawn from a uniform distribution (limits o; 1).

\section{Calculation of quality of life}

In the simulation, QoL is re-calculated at every event, based on the visual acuity of the better-seeing eye $\left(V A_{b}\right)$ and the parameters from the linear regression analysis from the quality of life study:

$\mathrm{QoL}=c+d * V A_{b}$

Where

$c \quad=$ the constant from the linear regression (0.270);

$d \quad=$ the linear regression coefficient (0.004);

$V A_{b}=$ visual acuity in the better-seeing eye. 


\section{References}

1. Radner W, Willinger U, Obermayer W, Mudrich C, Velikay-Parel M, Eisenwort B. [A new reading chart for simultaneous determination of reading vision and reading speed]. Klin Monbl Augenheilkd 1998;213:174-181.

2. Maaijwee KJ, Meulendijks CF, Radner W, van Meurs JC, Hoyng CB. [The Dutch version of the Radner Reading Chart for assessing vision function]. Ned Tijdschr Geneeskd 2007;151:2494-2497.

3. Charlson M, Pompei P, Ales K, MacKenzie C. A new method of classifying prognostic comorbidity in longitudinal studies: development and validation. J Chronic Dis 1987;40:373-383.

4. Elshout M, van der Reis MI, Webers CA, Schouten JS. The cost-utility of aflibercept for the treatment of age-related macular degeneration compared to bevacizumab and ranibizumab and the influence of model parameters. Graefes Arch Clin Exp Ophthalmol 2014.

5. Van Gestel A, Severens JL, Webers CA, Beckers HJ, Jansonius NM, Schouten JS. Modeling complex treatment strategies: construction and validation of a discrete event simulation model for glaucoma. Value Health 2010;13:358-367.

6. Wong TY, Chakravarthy U, Klein R, et al. The natural history and prognosis of neovascular age-related macular degeneration: a systematic review of the literature and meta-analysis. Ophthalmology 2008;115:116-126.

7. Willekens F. Continuous-time Microsimulation in Longitudinal Analysis. In: Zaidi AH, A. Williamson, P. (ed), New Frontiers in Microsimulation Modelling. Vienna, Ashgate; 2009. 
4.
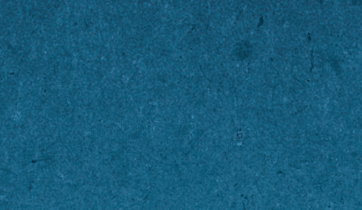

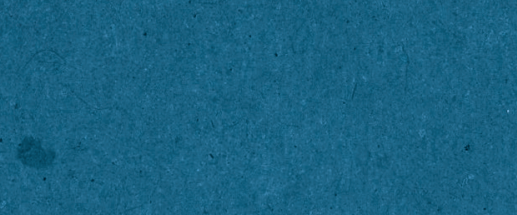
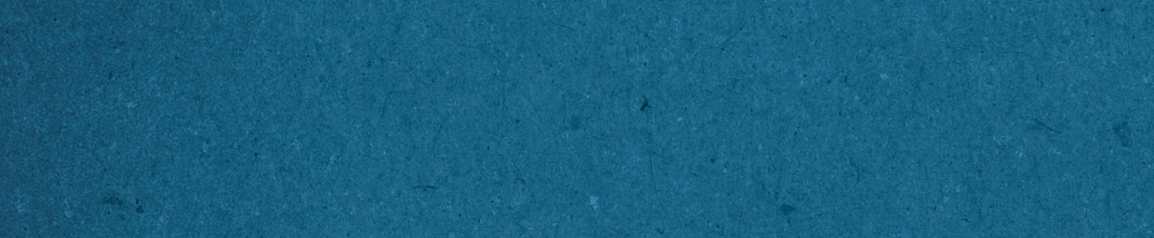

8

$y 3$

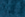




\section{Chapter 3}

\section{A systematic review on the quality, validity and usefulness of current cost-effectiveness studies on the treatment for neovascular age-related macular degeneration}

Mari Elshout, Carroll A.B. Webers, Margriet I. van der Reis, Jan S.A.G. Schouten Acta Ophthalmologica 2018; 96: 770-778 


\section{Abstract}

Purpose: Ophthalmologists increasingly depend on new drugs to advance their treatment options. These options are limited by restraints on reimbursements for new and expensive drugs. These restraints are put in place through health policy decisions based on cost-effectiveness analyses (CEA). Cost-effectiveness analyses need to be valid and of good quality to support correct decisions to create new treatment opportunities. In this study, we report the quality, validity and usefulness of CEAs for therapies for neovascular age-related macular degeneration (nAMD).

Methods: A systematic review in PubMed, Embase, and Cochrane was performed to include CEAs. Quality criteria were based on current general quality criteria and on elements that are specific to the field of ophthalmology.

Results: Forty-eight CEAs were included in the review. Forty-four CEAs did not meet four basic model quality and validity criteria specific to CEAs in the field of ophthalmology (both eyes analysed instead of one; a time horizon extending beyond four years; extrapolating VA and treatment intervals beyond trial data realistically; and including the costs of low-vision). Four CEAs aligned with the quality and validity criteria. In two of these CEAs, bevacizumab as-needed (PRN) was more cost-effective than bevacizumab monthly; aflibercept (VIEW); or ranibizumab monthly or PRN. In two CEAs, ranibizumab (PRN or treat and extent) was dominant over aflibercept. In two other CEAs, aflibercept was either more cost-effective or dominant over ranibizumab monthly or PRN.

Conclusion: Two of the CEAs of sufficient quality and validity show that bevacizumab PRN is the most cost-effective treatment. Comparing ranibizumab and aflibercept, either treatment can be more cost-effective depending on the assumptions used for drug prices and treatment frequencies. The majority of the published CEAs are of insufficient quality and validity. They wrongly inform decision-makers at the cost of opportunities for ophthalmologists to treat patients. As such, they may negatively influence overall patient outcomes and societal costs. For future ophthalmic treatments, CEAs need to be improved and only published when they are of sufficient quality and validity. 


\section{Introduction}

Several treatments are available for neovascular age-related macular degeneration (nAMD). Modern treatments are intravitreal injections with anti-vascular endothelial growth factors (anti-VEGF) aflibercept, ranibizumab, and bevacizumab. They are relatively similar in their effect in improving vision. Earlier treatments are generally less effective and include photodynamic therapy (PDT); intravitreal injections with the anti-VEGF pegaptanib and laser photocoagulation. Associated costs differ between the treatments. Concern has been raised regarding the increasing direct and indirect costs associated with nAMD, ranging from 1,000 to more than 10,000 euros per case per year.1, ${ }^{2}$ Furthermore, newer treatments such as intravitreal aflibercept have become available. Therefore, several cost-effectiveness analyses (CEA) comparing the treatments of nAMD have been conducted and published.

A CEA is a comparison of two or more interventions. The costs as well as the effectiveness of the interventions are compared. Analyses that measure effectiveness in natural or clinical units such as life-years saved or number of cases of blindness prevented, are commonly called "cost-effectiveness analyses," whereas those reporting quality-adjusted life-years (QALY) are also referred to as "cost-utility analyses (CUA)."3 Interpreting the results of CEAs and CUAs requires the definition of several specific concepts in their methodology as described below.

\section{Quality of life and quality-adjusted life-years}

Quality of life (QoL) can be regarded as a broad, generic measure of most functions of a persons' daily activities and well-being. These can be measured with QoL questionnaires such as the NEI-VFQ-25. Sometimes the QoL measure can be regarded to be a "utility". These can also be assessed using standardised questionnaires, providing a score typically ranging from $\mathrm{o}$ to 1 . Examples of generic utility measures are the health utilities index mark 3 (HUI-3) $)^{5}$ and the "time-trade off" method., In a CUA, QoL valuations are often used from other studies, and are expressed longitudinally with QALYs. While the outcomes of a treatment in a CEA is expressed usually with a clinical measure, such as visual acuity, the CUA, as a special case of CEA uses the QALY as the outcome. One QALY is the equivalent of living one year with a QoL of 1, or "100\%". The QALY combines time and QoL (expressed as utility) in one metric and thus allows for comparison of outcomes between different treatments for a disease, and even between treatments for different diseases. 


\section{Simulation models}

Cost-effectiveness analyses require data about resource use and health effects, often over a longer time span. Such data are not always readily available from observational or experimental studies, because clinical trials may have a short follow-up, compare two or three isolated treatment options and often do not collect data on resource use or quality of life. Cost-effectiveness analyses typically use computerized simulation models to aggregate the necessary pieces of scientific, clinical, and economic information. ${ }^{8}$

\section{The incremental cost-effectiveness ratio}

In comparing a treatment to a comparator, a CEA reports an incremental cost-effectiveness ratio (ICER). The ICER is calculated by taking the difference in costs between the treatments, and dividing this by the difference in the outcome (e.g. QALYs). For comparing treatment $A$ and $B$, this gives: ICER $=\left(\operatorname{costs}_{A}-\operatorname{costs}_{B}\right) /\left(Q_{A L Y}-Q_{A} A L Y\right)$. In some instances, treatment A may prove both more effective and less costly than treatment $\mathrm{B}$. In this case, treatment A can then be reported as to "dominate" treatment B.

Thus, the incremental cost and incremental effectiveness of an intervention are calculated relative to a comparator. A ratio (ICER) of the costs and effectiveness is computed: the numerator of the ratio is generally the net resource consumption. The denominator can be e.g. years of life saved or quality-adjusted life-years (QALYs) saved.

Quality, validity and usefulness of a CEA are important to expand the opportunities to treat patients. Policy makers use CEAs to make important decisions regarding the reimbursement of treatments, directly affecting patients, practitioners and clinics. A government or third-party insurer may decide to discontinue reimbursement of a drug if a CEA showed it was not cost-effective. Not only the results of CEAs, but also the quality and validity of their approach need to be assessed to justify important decisions. This review addresses CEAs of photodynamic therapy with verteporfin (PDT), laser photocoagulation, intravitreal injections with aflibercept $\left(\right.$ Eylea $\left.^{\circledR}\right)$ pegaptanib $\left(\right.$ Macugen $\left.^{\circledR}\right)$, ranibizumab (Lucentis ${ }^{\circledR}$ ) and bevacizumab $\left(\right.$ Avastin $\left.^{\circledR}\right)$ for nAMD. Our study questions were: What is the quality of current CEAs? Do current CEAs use models that are valid? What are the results of the valid CEAs? What should be improved in future CEAs? We reported the quality of the methodology and reporting and validity of the models. We also addressed issues that are specific to and vital for the validity of ophthalmic CEAs and that are not addressed by generic CEA quality criteria. 


\section{Materials and Methods}

\section{Search}

We conducted a systematic search for CEAs for therapies of nAMD in January 2017. The review was restricted to the following treatments: aflibercept, PDT, laser photocoagulation, pegaptanib, ranibizumab and/or bevacizumab. We used the databases PubMed, Embase, and the Cochrane database of Economic Evaluations. No date limits were imposed. Results were restricted to papers in English, French, German or Dutch. We used the following search terms in accordance with recommendations from several sources. . $^{-11}$ In PubMed: ("Costs and Cost Analysis"[Mesh] OR costs OR cost OR "economics "[Subheading] OR economic* OR cost-effective*[tw] OR cost-utility[tw] OR cost-benefit[tw]) AND ("Macular Degeneration"[Mesh] OR macula*[tw] OR AMD[tw] OR ARMD[tw]); In EMBASE: (macula* or AMD or ARMD).ti,ab.) and (exp economic aspect/ or cost*.tw). In the Cochrane database: macula* or AMD or ARMD.

\section{Selection}

We assessed titles and abstracts of the articles returned by the search. Articles unrelated to $\mathrm{nAMD}$ or not including costs in the study were excluded. The remaining articles were in- or excluded after reading and assessing the full-text.

Inclusion criteria were:

Study design: cost-effectiveness analysis (this also includes CUAs): an economic analysis that assesses both costs and outcomes of a treatment.

Interventions and comparator treatments: comparison of (combinations of) aflibercept, laser photocoagulation, PDT, pegaptanib, bevacizumab, ranibizumab, and natural history (placebo, 'usual care', or 'no treatment').

Disease: Subfoveal choroidal neovascularisation due to nAMD.

\section{Quality assessment}

We scored the quality of the articles on a 35-item checklist developed for the British Medical Journal (BMJ) to assess the quality of economic evaluations.12 On this generic checklist, higher scores suggest lower quality.

\section{Validity criteria specific to CEAs in ophthalmology}

In addition to the above quality assessment, we assessed the CEAs according to additional validity criteria. We applied four criteria vital to the validity of ophthalmic CEAs specifically. These are not usually addressed in quality assessment checklists, 
but affect outcomes of the CEA to an important degree. They focus not on the quality and transparency of the manuscript but assess as whether the nAMD as a disease was adequately modeled. As a first criterion: Was a two-eye analysis conducted? A one-eye approach is also known as a better-seeing eye analysis, which assumes the other, nontreated eye is already blind. Here, a two-eye model would be more realistic. A two-eye model includes the course of VA in each eye of the patient. With nAMD being a bilateral disease, it affects both eyes over the longer term, and QoL is not significantly impaired until the second eye becomes affected, while costs have already been incurred. From this follows the second criterion: Was the time-horizon long enough? A long time horizon in a model, allows nAMD as a bilateral disease to affect both eyes in a relevant proportion of patients, and thus have the treatment make a difference in terms of QoL. Therefore, the time horizon should be as long as possible. On the other hand, the time-horizons that are employed in the literature are limited. Description of the natural history of nAMD beyond four years is rare.13,14 Therefore, we believe that the time-horizon of the CEA ideally should be four years (with little to no extrapolation of disease progression needed) or more (with proper extrapolation). This leads to a third criterion: Was the course of visual acuity and treatment frequency extrapolated in a realistic manner? An example of an unrealistic approach of extrapolating beyond trial data, is one that assumes VA to remain stable without treatment, sometimes known as last observation carried forward (LOCF). A more realistic approach would be to assume, for example, a natural history course of VA when treatment is stopped. As a fourth criterion, it is essential for ophthalmologic CEAs to include the cost of low-vision or legal blindness. Without including these potentially substantial indirect costs in a CEA, a treatment that is actually cost-effective may be found to be not cost-effective.

\section{Data extraction}

From the CEAs that complied with the four additional validity criteria described above, we extracted the intervention; the comparator treatment; and the incremental costeffectiveness ratio (ICER). 


\section{Results}

From 2709 unique records from the three databases, a total of forty-eight CEAs were identified. Figure 1 shows a flow chart of the systematic review process. Table 1 shows the quality scores of the CEAs on the BMJ quality score list for economic studies. Furthermore, Table i specifies whether the CEA passed the four validity criteria described in the Methods section. Quality scores ranged from o to 14, with an average of 5.3. Regarding the four additional quality and validity criteria, strikingly, 42 CEAs used a one-eye model or did not specify the type of model used, and therefore failed the two-eye model validity criterion. More CEAs complied with the time-horizon criterion: 38 CEAs had a time horizon of four years more. Of these, 20 CEAs extrapolated the VA course and treatment intervals using a realistic method. The costs of blindness or low-vision such as rehabilitation were not included by 19 CEAs causing these CEAs to fail this validity criterion of including costs of low-vision. Four CEAs complied with all validity criteria: Claxton et al, ${ }_{15}^{15}$ Elshout et al, ${ }^{16}$ Ghosh et al, ${ }^{17}$ and Vottonen \& Kankaanpää. ${ }^{18}$ These CEAs used two-eye models, used a time-horizon of four years or more, included the indirect costs of low vision or blindness due to $\mathrm{nAMD}$, and extrapolated VA and treatment intervals beyond trial durations using realistic methods. These CEAs had a quality score of four or less. Thus, these CEAs were selected for having sufficient report quality and model validity in order to ascertain that the results reported were sufficiently reliable and useful.

All four CEAs were of the CUA type: effectiveness was reported in QALYs. Three of the four CEAs used a patient-level model type, in which virtual patients are simulated one by one, with, for each patient, characteristics of each eye recorded and with the opportunity to change as the model runs. ${ }^{15-17}$ Markov simulation depends on transition probabilities between pre-set health states. Because individual patients cannot be traced in Markov simulation, developing a two-eye Markov model can be challenging. One study developed a Markov model to accommodate two eyes, by defining health states that describe the health states of each eye of the patients in that state, e.g. "One eye is healthy, the other eye has wAMD and visual acuity is normal".18 Time horizons were five years in the models of Elshout and Ghosh; eight years and lifetime time horizons were used in the models of Vottonen and Claxton, respectively. Both Claxton and Ghosh modeled clinical effectiveness of ranibizumab versus aflibercept using a network meta-analysis of randomised controlled trials. Regarding modeling of the clinical effectiveness of the treatments compared, both Elshout and Vottonen used visual acuity data from several trials for the effectiveness of aflibercept, bevacizumab, and ranibizumab, which also underpinned the monitoring and treatment intervals in the models (see Table 2). Table 2 lists the perspectives employed by the study and which type of costs they included in their models. As both Claxton and Ghosh used the perspective of the UK National Health 
Service (NHS), both included the same costs for treatment, monitoring, and for blindness (such as rehabilitation costs, which are also covered by the NHS). Elshout, assuming the societal perspective, also included the costs for informal care and for adverse events but did not include the costs of hip surgery or depression. Vottonen assumed a more restricted hospital perspective and therefore did not include the costs of home care. Table 2 also lists results extracted from the publications: the treatment strategies compared; the source for the follow-up and treatment schedules; the cost prices per injection in the CEA; and the corresponding ICER. All four CEAs included aflibercept treatment: 8-weekly for one year followed by as-needed (pro re nata, PRN), as in the VIEW 1 and 2 studies. ${ }^{19}$ Claxton, Ghosh, and Vottonen compared aflibercept with ranibizumab PRN; treat-andextent (T\&E); and both monthly and PRN, respectively. Elshout compared aflibercept and ranibizumab (monthly and PRN) to no treatment, without a direct comparison. Claxton and Ghosh found that ranibizumab dominated aflibercept: ranibizumab being less costly and equally or slightly more effective. Both CEAs priced aflibercept higher than ranibizumab in their analysis. On the other hand, Vottonen found negative ICERS of aflibercept over ranibizumab: here, aflibercept dominated ranibizumab, with ranibizumab priced significantly higher than aflibercept as opposed to the assumptions by Claxton and Ghosh. Elshout found a lower ICER with aflibercept than ranibizumab, monthly or PRN: aflibercept was more cost-effective than ranibizumab (over no treatment). In this CEA, the lower number of total injections needed for aflibercept versus ranibizumab appeared to compensate for a higher price for aflibercept.

Aflibercept was also compared to bevacizumab (monthly and PRN) by Vottonen. Aflibercept had high ICERs (almost 1 million euros or more) over bevacizumab, it was not cost-effective over bevacizumab. Disaggregated results showed the difference in QALY yield per patient was less than 0.03 between treatments. Total costs, however, where significantly lower for bevacizumab than for aflibercept ( $>50 \%$ lower) and for ranibizumab (>80\% lower). Elshout compared bevacizumab to no treatment, with bevacizumab PRN (as in the $\mathrm{ABC}$ study ${ }^{2 \circ}$ ) yielding the lowest ICER, followed by bevacizumab PRN (as in the CATT study ${ }^{21}$ ); and monthly bevacizumab. Aflibercept; ranibizumab PRN; and monthly ranibizumab, yielded increasingly higher ICERs. This means that bevacizumab PRN was more cost-effective than monthly bevacizumab, and more cost-effective than ranibizumab (monthly or PRN), or aflibercept. 


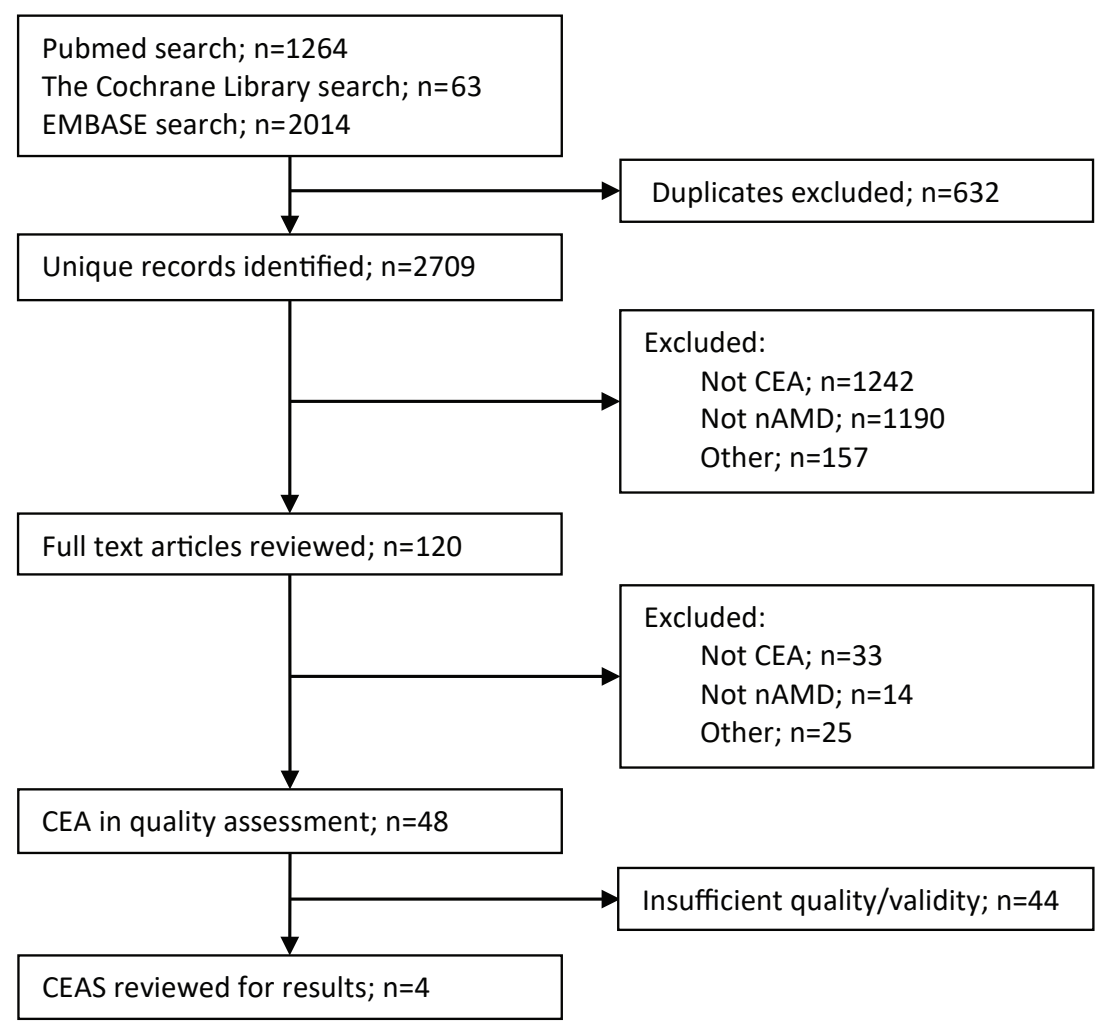

Figure 1. Selection of studies in review of cost-effectiveness analyses regarding treatments of neovascular age-related macular degeneration. CEA, cost-effectiveness analysis; $n A M D$, neovascular age-related macular degeneration. 
Table 1. Methodology, quality and validity assessment of cost-effectiveness analyses for the treatment of neovascular age-related macular degeneration.

\begin{tabular}{|c|c|c|}
\hline \multirow{3}{*}{ Study Reference } & \multicolumn{2}{|c|}{ Model } \\
\hline & Type & QoL Valuation in CUA \\
\hline & & \\
\hline Wolowacz $^{23}$ & Markov & $\mathrm{TTO}^{22}$ \\
\hline Claxton $^{15}$ & Patient-level & $\mathrm{TTO}^{24}$ \\
\hline Elshout $^{16}$ & Patient-level & HUI-3 (184 patients) \\
\hline Brown $^{6}$ & NR & TTO (233 patients) \\
\hline Meads 25 & Decision tree & $\mathrm{TTO}^{22}$ \\
\hline Smith $^{26}$ & Markov & $\mathrm{TTO}^{22}$ \\
\hline Ghosh $^{17}$ & Patient-level & $\mathrm{TTO}^{24}$ \\
\hline Busbee $^{27}$ & Markov & $\mathrm{TTO}^{7}$ \\
\hline Larouche $^{28}$ & Markov & $\mathrm{TTO}^{22}$ \\
\hline Dakin $^{29}$ & NA (CUA within trial) & EQ-5D (within trial) \\
\hline Hernández-Pastor ${ }^{30}$ & Markov & $\mathrm{TTO}^{22}$ \\
\hline Neubauer $^{31}$ & Markov & $\mathrm{TTO}^{22}$ \\
\hline Colquitt $^{32}$ & Markov & $\mathrm{TTO}^{22}$ \\
\hline Brown ${ }^{33}$ & Markov & $\mathrm{TTO}^{34}$ \\
\hline Earnshaw $^{35}$ & Markov & $\mathrm{TTO}^{22}$ \\
\hline Javitt ${ }^{36}$ & Markov & $\mathrm{TTO}^{22}$ \\
\hline Hernández-Pastor ${ }^{37}$ & Markov & $\mathrm{TTO}^{22}$ \\
\hline Yanagi $^{38}$ & Markov & $\mathrm{TTO}^{39}$ \\
\hline Vottonen $^{18}$ & Markov & $\mathrm{TTO}^{22}$ \\
\hline Patel $^{40}$ & Markov & $\mathrm{TTO}^{22}$ \\
\hline Sharma ${ }^{41}$ & Markov & $\mathrm{TTO}^{22}$ \\
\hline Grieve $^{42}$ & NA: Observational data & SF-6D ${ }^{43}$ \\
\hline Panchmatia ${ }^{44}$ & Markov & $\mathrm{TTO}^{24}$ \\
\hline $\mathrm{Wu}^{45}$ & Markov & $\mathrm{TTO}^{22}$ \\
\hline $\mathrm{Butt}^{46}$ & Markov & $\mathrm{TTO}^{22}$ \\
\hline Studnicka ${ }^{47}$ & NA: Retro-spective study & NA (CEA) \\
\hline Brown $^{48}$ & Decision tree & $\mathrm{TTO}^{7}$ \\
\hline Butt 49 & Markov & SF-6D 5 \\
\hline Hurley $^{50}$ & Markov & $\mathrm{TTO}^{22}$ \\
\hline Stein $^{51}$ & Markov & $\mathrm{NR}^{52}$ \\
\hline Gower $^{53}$ & Markov & $\mathrm{TTO}^{54}$ \\
\hline Brown $^{55}$ & Markov & TTO (100o eye patients) \\
\hline Meads $^{56}$ & Decision tree & $\mathrm{TTO}^{22}$ \\
\hline Athanasakis ${ }^{57}$ & Markov & $\mathrm{TTO}^{22}$ \\
\hline Brown $^{58}$ & NR & $\mathrm{TTO}^{6}$ \\
\hline Neubauer $^{59}$ & NR & $\mathrm{TTO}^{60,63}$ \\
\hline Smiddy $^{61}$ & NR & NA (CEA) \\
\hline Raftery $^{62}$ & Markov & $\mathrm{TTO}^{22}$ \\
\hline Hurley $^{50}$ & Markov & $\mathrm{TTO}^{22}$ \\
\hline Bansback $^{63}$ & Markov & $\mathrm{HUI}-3^{5}$ \\
\hline Fletcher $^{64}$ & Decision tree & $\mathrm{TTO}^{34}$ \\
\hline
\end{tabular}




\begin{tabular}{|c|c|c|c|c|}
\hline Quality & \multicolumn{4}{|c|}{ Validity of Model } \\
\hline & 1- or 2-Eye model & Time Horizon, y & Costs of Low & Realistic VA \\
\hline & & & Vision Included & Extrapolation \\
\hline b & 1 & 10 & $\mathrm{Y}$ & $\mathrm{Y}$ \\
\hline * & $1 \& 2$ & Life & $\mathrm{Y}$ & Y \\
\hline * & 2 & $2 ; 5$ & $\mathrm{Y}$ & $\mathrm{Y}$ \\
\hline 2 & 1 & 12 & $\mathrm{~N}$ & $\mathrm{~N}$ \\
\hline 2 & 1 & 2 & $\mathrm{Y}$ & NA \\
\hline 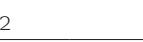 & 1 & $2 ; 5$ & $\mathrm{Y}$ & NR \\
\hline * & 2 & 5 & $\mathrm{Y}$ & $\mathrm{Y}$ \\
\hline 3 & 2 & Life (14) & $\mathrm{N}$ & Y \\
\hline 3 & 2 & 8 & Y & $\mathrm{N}$ \\
\hline 3 & 1 & 2 & $\mathrm{~N}$ & NA \\
\hline 3 & 1 & 2; Life & $\mathrm{N}$ & $\mathrm{Y}$ \\
\hline 3 & 1 & 10 & Y & $\mathrm{N}$ \\
\hline 3 & 1 & $1 ; 2 ; 10$ & Y & Y \\
\hline 3 & 1 & Life & $\mathrm{Y}$ & NR \\
\hline 3 & 1 & Life & $\mathrm{Y}$ & $\mathrm{Y}$ \\
\hline 3 & 1 & Life & Y & $\mathrm{Y}$ \\
\hline 3 & 1 & Life & $\mathrm{Y}$ & $\mathrm{Y}$ \\
\hline 3 & 1 & 12 & $\mathrm{Y}$ & $\mathrm{Y}$ \\
\hline * & 2 & 8 & $\mathrm{Y}$ & $\mathrm{Y}$ \\
\hline 4 & NR & 20 & $\mathrm{~N}$ & NR \\
\hline 4 & 1 & 2,11 & $\mathrm{~N}$ & $\mathrm{Y}$ \\
\hline 4 & 1 & 2 & $\mathrm{Y}$ & NA \\
\hline 4 & 1 & Life & $\mathrm{Y}$ & $\mathrm{N}$ \\
\hline 4 & 1 & Life & $\mathrm{Y}$ & NR \\
\hline 5 & 1 & 2 & $\mathrm{~N}$ & NA \\
\hline 5 & 1 & 2 & $\mathrm{~N}$ & NA \\
\hline 5 & 1 & 4 & $\mathrm{~N}$ & $\mathrm{Y}$ \\
\hline 5 & 1 & 5 & $\mathrm{~N}$ & Y \\
\hline 5 & 1 & $2 ; 10$ & $\mathrm{Y}$ & $\mathrm{Y}$ \\
\hline 5 & 1 & $2 ; 5$ & $Y$ & NR \\
\hline 5 & 1 & 2 & $\mathrm{~N}$ & NA \\
\hline 5 & $1 \& 2$ & 12 & $\mathrm{~N}$ & $\mathrm{~N}$ \\
\hline 5 & 1 & 1 & $\mathrm{Y}$ & NA \\
\hline 8 & 1 & 10 & $\mathrm{~N}$ & NR \\
\hline 8 & 1 & 12 & $\mathrm{~N}$ & $\mathrm{~N}$ \\
\hline 7 & 1 & 10 & $\mathrm{Y}$ & $\mathrm{N}$ \\
\hline 3 & NR & $1 ; 2$ & $\mathrm{~N}$ & NA \\
\hline 3 & 1 & 10 & $\mathrm{Y}$ & $\mathrm{Y}$ \\
\hline 3 & 1 & $2 ; 10$ & Y & $\mathrm{Y}$ \\
\hline 3 & 1 & $2 ; 5 ; 10$ & $\mathrm{Y}$ & $\mathrm{Y}$ \\
\hline 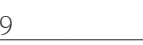 & 1 & 5 & $\mathrm{~N}$ & NR \\
\hline
\end{tabular}


Table 1. Continued.

\begin{tabular}{lcc} 
& \multicolumn{2}{c}{ Model } \\
\cline { 2 - 3 } Study Reference & Type & QoL Valuation in CUA \\
\hline Smiddy $^{65}$ & & NA: CEA \\
\hline Cohen $^{66}$ & Decision tree & NA: CEA \\
\hline Greiner $^{67}$ & Markov & NA: CEA \\
\hline Hopley $^{68}$ & Decision analysis & TTO \\
\hline Sharma $^{69}$ & NR & TTO (224 patients) \\
\hline Donati $^{70}$ & Markov & TTO $^{6}$ \\
\hline Muslera $^{71}$ & NR & TTO $^{22}$ \\
\hline
\end{tabular}

* Study met all 4 additional quality and validity criteria.

CEA, cost-effectiveness analysis; CUA, cost-utility analysis; HUI-3, Health Utilities Index Mark 3;

Table 2. Results of cost-effectiveness analyses for the treatment of neovascular age-related macular degeneration.

Study (Country, Perspective)

Claxton et al., two-eye model (UK, NHS perspective)

Elshout et al., 5-year model

(The Netherlands, societal perspective)

Ghosh et al.

(UK, NHS perspective)

Vottonen \& Kankaanpää

(Finland, hospital perspective)

\section{Costs Included}

drugs; administration; monitoring (staff, OCT); blindness (home care, depression, aids, rehab, registration, hip surgery) drugs; administration; diagnosis and monitoring (staff, OCT, FA); adverse events (endophthalmitis, retinal detachment, lens injury); blindness / low-vision (home care, informal care, aids, rehab, moving house)

drugs; administration; monitoring (staff, OCT); blindness (home care, depression, aids, rehab, registration, hip surgery)

drugs; administration; monitoring (staff, OCT, FA); adverse events (endophthalmitis, retinal detachment, lens injury); blindness (rehab, aids, informal care)

\footnotetext{
* All four cost-effectiveness analyses in this table where of the cost-utility analysis type. 8w/PRN, 8-weekly in year 1 followed by as needed; FA, fluorescein angiography;
} 


\begin{tabular}{|c|c|c|c|c|}
\hline \multirow{3}{*}{$\frac{\text { Quality }}{\text { Score }}$} & \multicolumn{4}{|c|}{ Validity of Model } \\
\hline & 1- or 2-Eye model & Time Horizon, y & Costs of Low & Realistic VA \\
\hline & & & Vision Included & Extrapolation \\
\hline 9 & NR & 10 & $\mathrm{~N}$ & NR \\
\hline 9 & 1 & 1 & $\mathrm{Y}$ & NA \\
\hline 9 & NR & 5 & $\mathrm{Y}$ & NR \\
\hline 10 & 1 & 7 & $\mathrm{~N}$ & $\mathrm{Y}$ \\
\hline 10 & 1 & 1 & Y & NA \\
\hline 13 & 1 & 5 & $\mathrm{Y}$ & NR \\
\hline 14 & NR & 2; Life & $\mathrm{N}$ & NR \\
\hline
\end{tabular}

$N A$, not applicable; $N R$, not reported; QoL, quality of life; $S F-6 D$, Short Form 6D Health Status

Questionnaire; TTO, time trade-off; VA, visual acuity.

\section{Treatment:}

Source of Monitoring and Treatment Intervals (drug cost per injection)

Intervention Comparison
Incremental

Cost-Effectiveness Ratio (Cost / QALY)*

Aflibercept 8w/PRN: VIEW ${ }^{19}$ ( $£ \quad$ Ranibizumab PRN: National UK Ranibizumab PRN is dominant

816)

Aflibercept 8w/PRN:

VIEW (€ 907)

Bevacizumab PRN:

$$
\mathrm{ABC}^{20}(€ 38)
$$

Bevacizumab PRN:

\section{CATT $^{21}$}

Bevacizumab monthly: CATT

Ranibizumab PRN:

CATT (€ 773)

Ranibizumab monthly:

MARINA $^{73}$

Ranibizumab T\&E:

Berg et al.74 (£ 816)

Aflibercept 8w/PRN

VIEW (€ 693) $\operatorname{study}^{72}(£ 742)$

No treatment:

Literature Review

No treatment $\quad € 51,062$

No treatment $€ 83,256$

No treatment $\quad € 110,361$

No treatment $\quad € 181,667$

No treatment $\quad € 349,773$

Aflibercept 8w/PRN: Ranibizumab

VIEW (£ 551) T\&E is dominant

Bevacizumab monthly $€ 1,801,228$

CATT $(€ 61)$

Bevacizumab PRN: CATT $\quad € 928,040$

Ranibizumab monthly $\quad €-14,040,620$

CATT (€ 1336)

Ranibizumab PRN $\quad €-3,716,943$

CATT

NHS, National Health Service; OCT, optical coherence tomography; $P R N$, pro re nata (as needed);

$Q A L Y$, quality-adjusted life-year; rehab, rehabilitation, $T \& E$, treat and extend. 


\section{Discussion}

We have performed a systematic review of CEAs regarding the cost-effectiveness of the current therapies for nAMD. We assessed the quality and validity of the CEAs. We could summarise the results of four CEAs that were of adequate quality and validity.

What is the quality of current CEAs? Quality scores on a general checklist ranged from o to 14, on a scale of o (higher quality) to 35 (lower quality). No cutoff exists for the quality being adequate. Although a number of studies have quality scores that appear reasonably adequate, a number of additional quality and validity criteria specific for CEAs in ophthalmology are needed.

Do current CEAs use models that are valid? Four cases of CEAs out of forty-eight used models valid for ophthalmology. Only these four CEAs complied with our additional quality / validity criteria specific to the field of ophthalmology: using a two-eye model; using a sufficiently long time-horizon; using proper methods of extrapolating beyond trial data; and including the costs of blindness and low-vision (indirect costs).

What are the results of the valid CEAs? Two CEAs found bevacizumab PRN to be more cost-effective than monthly bevacizumab; aflibercept (8-weekly for 1 year followed by PRN: VIEW); and ranibizumab monthly or PRN. Results of comparisons between aflibercept and ranibizumab were different between CEAs. Contradicting outcomes could be explained by different assumptions regarding cost prices for the medications and the reference trials used for the frequency of their use. Two CEAs found ranibizumab PRN or T\&E to be dominant over aflibercept treatment (as in the VIEW studies). The two other CEAs found that aflibercept was more cost-effective or dominant over ranibizumab monthly or PRN.

What should be improved in future CEAs? A majority of reviewed CEAs used a one-eye model to simulate disease progression. Such a model is an oversimplification of nAMD as a bilateral disease. It assumes that patients have only one eye that is treated. The other eye is not included in the analysis or is assumed to have a lower VA and is not treated. In quality of life research, it has been shown that the VA in the better eye predominantly determines quality of life. ${ }^{22}$ In one-eye models, treatment thus leads directly to an improvement in quality of life (Panel A in Figure 2). In two-eye models, more similar to clinical practice, the worst eye is most often the eye being treated initially (Panel B in Figure 2). The quality of life is not significantly changed by the treatment of the worst eye. However, this eye determines most of the total costs of treatment. It is realistic that initially, costs are incurred while no gain in quality of life is acquired. Only after the better 
eye develops nAMD and is treated, the treatment starts to make a difference in QALYs lost or gained. More direct costs are incurred, as both eyes are eventually treated. A twoeye model will yield less incremental QALYs and more costs for a treatment. This leads to a higher, yet more realistic ICER.

A

One-eye model
B

Two-eye model

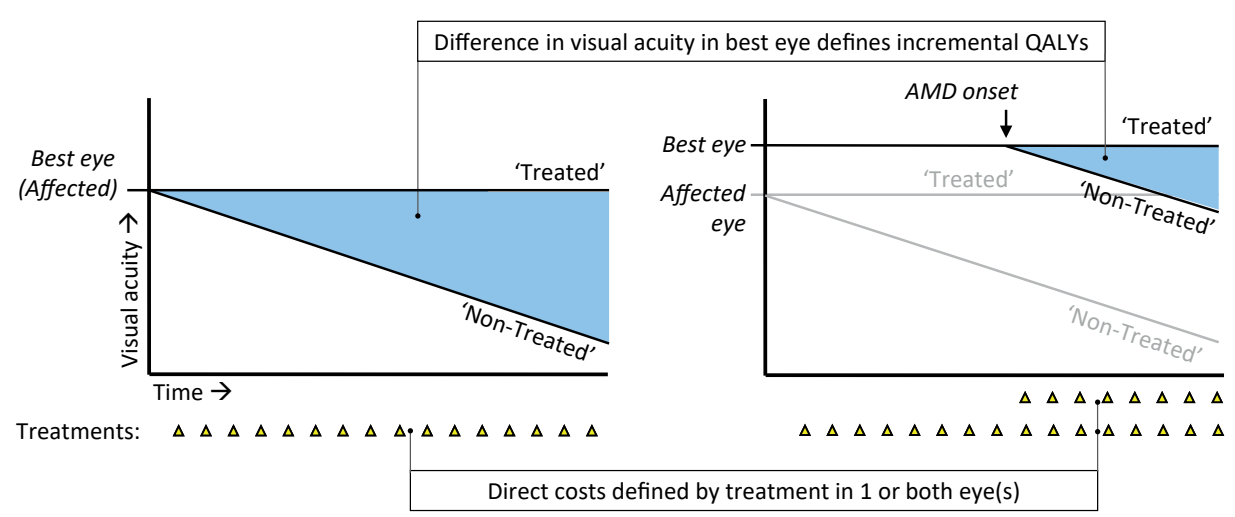

Figure 2. Schematic comparison of one-eye (A) and two-eye (B) models: Difference in incremental quality-adjusted life-years (QALYs) and direct costs. Shaded area: incremental QALYs (correlated with visual acuity in best eye). Triangles: Schematic relative number of treatment sessions (which largely define direct costs). $n A M D$, neovascular age-related macular degeneration.

Indirect costs such as the costs of vision loss should be included in an ophthalmic CEA as the costs of disability due to blindness or poor vision can be considerable. A more effective intervention leads to lower rates of vision loss. It thereby decreases indirect costs of blindness and decreases the ICER. Not including the cost of VA loss therefore causes a significant overestimation of the ICER (higher cost per QALY).

The choice of time horizon can affect the ICER, as extending the time horizon can alter incremental costs and/or incremental benefits. If, later on in the timeline, incremental QALYs are less and/or incremental costs are greater than earlier on, extending the time horizon can have an increasing effect on the ICER. On the other hand, when QALY gain is greater and/ or costs are lower later on in the timeline, extending the time horizon can have a decreasing effect on the ICER. 
As an illustration, in comparing VA-stabilising treatment to a scenario with decreasing VA (e.g. anti-VEGF versus no treatment), incremental QALYs may be greater in later years. Regarding two-eye models, the disease burden may be greater in later years, as more at-risk fellow eyes become affected. Here, treatment costs can also be greater later on, as both eyes may be treated eventually.

Developing a long-term model is needed but it is only warranted as long as the method of extrapolation is realistic. In many of the reviewed CEAs this is not the case. Last-observationcarried-forward is frequently used. This method of extrapolation confers bias to a CEA if the recurrence rate with vision loss after treatment is high, as it is in anti-VEGF treatment. It is not realistic when treatment is not continued over the period of extrapolation while assuming sustained VA, as is assumed in many CEAs. Applying vision loss similar to the natural progression of the disease, after the treatment ends, is an acceptable method. Keeping VA stable over time while maintaining the number of treatments per year as in the last year of the reference trial is another example of adequate extrapolation in anti-VEGF treatment.

In summary, future ophthalmic cost-effectiveness CEAs should address the following issues:

Two-eye models. Two-eye analyses are needed for realistic decision making in nAMD treatment;

A sufficiently long time horizon. The time horizon should be long enough to allow a significant number of fellow eyes to become affected with the disease and thus for the treatment to yield a clear difference in terms of QALYs. As both incremental costs and benefits may change over time, depending on the scenarios employed, a longer time horizon can alter the ICER;

Realistic extrapolation. The extrapolation of VA, the treatment and follow-up schedule should be realistic over the entire time horizon. Last-observation-carried-forward without active treatment should not be accepted as a realistic method of extrapolation beyond trial data;

Indirect costs. The costs of blindness or low-vision should be included in the analysis.

As most of current CEAs did not comply with the above criteria, they should not be used for decision making. Most of current economic evaluations of treatments for nAMD may wrongly inform decision-makers at the cost of opportunities for ophthalmologists to treat patients. As such, they may negatively influence overall patient outcomes and societal costs. More CEAs with adequate quality and validity are needed to further specify the cost-effectiveness of current treatments of nAMD. They would add to the current base of economic studies of treatments of $n A M D$, which at this point is very limited with the results on certain aspects still being contradictory. 


\section{References}

1. Cruess AF, Zlateva G, Xu X, et al. Economic burden of bilateral neovascular age-related macular degeneration: Multi-country observational study. PharmacoEconomics 2008;26:57-73.

2. Salm M, Belsky D, Sloan FA. Trends in cost of major eye diseases to Medicare, 1991 to 2000. Am J Ophthalmol 2006;142:976-982.

3. Tengs TO. Cost-effectiveness versus cost-utility analysis of interventions for cancer: does adjusting for health-related quality of life really matter? Value Health 2004;7:70-78.

4. Mangione CM, Lee PP, Gutierrez PR, Spritzer K, Berry S, Hays RD. Development of the 25-item National Eye Institute Visual Function Questionnaire. Arch Ophthalmol 2001;119:1050-1058.

5. Espallargues M, Czoski-Murray CJ, Bansback NJ, et al. The impact of age-related macular degeneration on health status utility values. Invest Ophthalmol Vis Sci 2005;46:4016-4023.

6. Brown GC, Brown MM, Campanella J, Beauchamp GR. The cost-utility of photodynamic therapy in eyes with neovascular macular degeneration--a value-based reappraisal with 5-year data. Am J Ophthalmol 2005;140:679-687.

7. Brown GC. Vision and quality-of-life. Trans Am ophthalmol soc 1999;97:473-511.

8. Brennan A, Akehurst R. Modelling in health economic evaluation. What is its place? What is its value? Pharmacoeconomics 2000;17:445-459.

9. Alton V, Eckerlund I, Norlund A. Health economic evaluations: how to find them. Int J Technol Assess Health Care 2006;22:512-517.

10. McKinlay RJ, Wilczynski NL, Haynes RB. Optimal search strategies for detecting cost and economic studies in EMBASE. BMC Health Serv Res 2006;6:67.

11. Stroup DF, Berlin JA, Morton SC, et al. Meta-analysis of observational studies in epidemiology: a proposal for reporting. Meta-analysis Of Observational Studies in Epidemiology (MOOSE) group. JAMA. United States; 2000:2008-2012.

12. Drummond MF, Jefferson TO. Guidelines for authors and peer reviewers of economic submissions to the BMJ. The BMJ Economic Evaluation Working Party. Bmj 1996;313:275-283.

13. Wong TY, Chakravarthy U, Klein R, et al. The natural history and prognosis of neovascular age-related macular degeneration: a systematic review of the literature and meta-analysis. Ophthalmology 2008;115:116-126.

14. Elshout M, Webers CA, van der Reis MI, de Jong-Hesse Y, Schouten JS. Tracing the natural course of visual acuity and quality of life in neovascular age-related macular degeneration: a systematic review and quality of life study. BMC ophthalmology 2017;17:120.

15. Claxton L, Hodgson R, Taylor M, Malcolm B, Pulikottil Jacob R. Simulation Modelling in Ophthalmology: Application to Cost Effectiveness of Ranibizumab and Aflibercept for the Treatment of Wet Age-Related Macular Degeneration in the United Kingdom. PharmacoEconomics 2016;1-12.

16. Elshout M, van der Reis MI, Webers CA, Schouten JS. The cost-utility of aflibercept for the treatment of age-related macular degeneration compared to bevacizumab and ranibizumab and the influence of model parameters. Graefes Arch Clin Exp Ophthalmol 2014;252:1911-1920.

17. Ghosh W, Wickstead R, Claxton L, et al. The Cost-Effectiveness of Ranibizumab Treat and Extend Regimen Versus Aflibercept in the UK. Advances in therapy 2016;33:1660-1676

18. Vottonen P, Kankaanpaa E. Cost-effectiveness of treating wet age-related macular degeneration at the Kuopio University Hospital in Finland based on a two-eye Markov transition model. Acta Ophthalmol 2016. 
19. Schmidt-Erfurth U, Kaiser PK, Korobelnik JF, et al. Intravitreal aflibercept injection for neovascular age-related macular degeneration: ninety-six-week results of the VIEW studies. Ophthalmology 2014;121:193-201.

20. Tufail A, Patel PJ, Egan C, et al. Bevacizumab for neovascular age related macular degeneration (ABC Trial): multicentre randomised double masked study. Bmj 2010;340:c2459.

21. Martin DF, Maguire MG, Fine SL, et al. Ranibizumab and bevacizumab for treatment of neovascular age-related macular degeneration: two-year results. Ophthalmology 2012;119:1388-1398.

22. Brown GC, Sharma S, Brown MM, Kistler J. Utility values and age-related macular degeneration. Arch Ophthalmol 2000;118:47-51.

23. Wolowacz SE, Roskell N, Kelly S, Maciver FM, Brand CS. Cost effectiveness of pegaptanib for the treatment of age-related macular degeneration in the UK. Pharmacoeconomics 2007;25:863-879.

24. Czoski-Murray C, Carlton J, Brazier J, Young T, Papo NL, Kang HK. Valuing condition-specific health states using simulation contact lenses. Value Health 2009;12:793-799.

25. Meads C, Salas C, Roberts T, Moore D, Fry-Smith A, Hyde C. Clinical effectiveness and cost-utility of photodynamic therapy for wet age-related macular degeneration: a systematic review and economic evaluation. Health Technol Assess 2003;7:V-vi, 1-98.

26. Smith DH, Fenn P, Drummond M. Cost effectiveness of photodynamic therapy with verteporfin for age related macular degeneration: the UK case. Br J Ophthalmol 2004;88:1107-1112.

27. Busbee BG, Brown MM, Brown GC, Sharma S. CME review: A cost-utility analysis of laser photocoagulation for extrafoveal choroidal neovascularization. Retina 2003;23:279-287; ; quiz 443-274.

28. Larouche K, Rochon S. Evaluation of photodynamic therapy for the treatment of exudative agerelated macular degeneration (ARMD) with subfoveal neovascularization (Structured abstract). Montreal: Agence d'Evaluation des Technologies et des Modes d'Intervention en Sante (AETMIS) 2004;101.

29. Dakin HA, Wordsworth S, Rogers CA, et al. Cost-effectiveness of ranibizumab and bevacizumab for age-related macular degeneration: 2-year findings from the IVAN randomised trial. BMJ open 2014;4:e005094.

30. Hernandez-Pastor LJ, Ortega A, Garcia-Layana A, Giraldez J. Cost-effectiveness of ranibizumab compared with photodynamic treatment of neovascular age-related macular degeneration. Clin Ther 2008;30:2436-2451.

31. Neubauer AS, Holz FG, Sauer S, et al. Cost-effectiveness of ranibizumab for the treatment of neovascular age-related macular degeneration in germany: Model analysis from the perspective of germany's statutory health insurance system. Clin Ther 2010;32:1343-1356.

32. Colquitt JL, Jones J, Tan SC, Takeda A, Clegg AJ, Price A. Ranibizumab and pegaptanib for the treatment of age-related macular degeneration: a systematic review and economic evaluation. Health Technol Assess 2008;12:iii-iv, ix-201.

33. Brown A, Hodge W, Cruess A, et al. Management of neovascular age-related macular degeneration: systematic drug class review and economic evaluation (Structured abstract). Ottawa: Canadian Agency for Drugs and Technologies in Health (CADTH) 2008;63.

34. Sharma S, Brown GC, Brown MM, et al. Converting visual acuity to utilities. Can J Ophthalmol 2000;35:267-272.

35. Earnshaw SR, Moride Y, Rochon S. Cost-effectiveness of pegaptanib compared to photodynamic therapy with verteporfin and to standard care in the treatment of subfoveal wet age-related macular degeneration in Canada. Clin Ther 2007;29:2096-2106; discussion 2094-2095. 
36. Javitt JC, Zlateva GP, Earnshaw SR, et al. Cost-effectiveness model for neovascular age-related macular degeneration: comparing early and late treatment with pegaptanib sodium based on visual acuity. Value Health 2008;11:563-574.

37. Hernandez-Pastor LJ, Ortega A, Garcia-Layana A, Giraldez J. Cost-effectiveness of ranibizumab compared with pegaptanib in neovascular age-related macular degeneration. Graefes Arch Clin Exp Ophthalmol 2010;248:467-476.

38. Yanagi Y, Fukuda A, Barzey V, Adachi K. Cost-effectiveness of intravitreal aflibercept versus other treatments for wet age-related macular degeneration in Japan. Journal of medical economics 2016;1-19.

39. Yanagi $Y$, Ueta T, Obata R, Iriyama A, Fukuda T, Hashimoto H. Utility values in Japanese patients with exudative age-related macular degeneration. Japanese journal of ophthalmology 2011;55:35-38.

40. Patel JJ, Mendes MA, Bounthavong M, Christopher ML, Boggie D, Morreale AP. Cost-utility analysis of bevacizumab versus ranibizumab in neovascular age-related macular degeneration using a Markov model. J Eval Clin Pract 2010.

41. Sharma S, Brown GC, Brown MM, Hollands H, Shah GK. The cost-effectiveness of photodynamic therapy for fellow eyes with subfoveal choroidal neovascularization secondary to age-related macular degeneration. Ophthalmology 2001;108:2051-2059.

42. Grieve R, Guerriero C, Walker J, et al. Verteporfin photodynamic therapy cohort study: report 3: cost effectiveness and lessons for future evaluations. Ophthalmology 2009;116:2471-2477 e2471-2472.

43. Reeves BC, Langham J, Walker J, et al. Verteporfin photodynamic therapy cohort study: report 2: clinical measures of vision and health-related quality of life. Ophthalmology. United States; 2009:2463-2470.

44. Panchmatia HR, Clements KM, Hulbert E, et al. Aflibercept vs. Ranibizumab: cost-effectiveness of treatment for wet age-related macular degeneration in Sweden. Acta Ophthalmol 2016;94:441-448.

45. Wu B, Li J, Lin H, Wu H. Different Strategies for the Treatment of Age-Related Macular Degeneration in China: An Economic Evaluation. Journal of ophthalmology 2016;2016:7689862.

46. Butt T, Lee A, Lee C, Tufail A. The cost-effectiveness of initiating ranibizumab therapy in eyes with neovascular AMD with good vision: an economic model using real-world outcomes. BMJ open 2015;5:e006535.

47. Studnicka J, Rihova B, Rencova E, et al. Cost and effectiveness of therapy for wet age-related macular degeneration in routine clinical practice. Ophthalmologica 2013;230:34-42.

48. Brown GC, Brown MM, Sharma S, Brown H, Tasman W. Incremental cost effectiveness of laser photocoagulation for subfoveal choroidal neovascularization. Ophthalmology 2000;107:1374-1380.

49. Butt T, Patel PJ, Tufail A, Rubin GS. Modelling cost effectiveness in neovascular age-related macular degeneration: the impact of using contrast sensitivity vs. visual acuity. Applied health economics and health policy 2014;12:289-297.

50. Hurley SF, Matthews JP, Guymer RH. Cost-effectiveness of ranibizumab for neovascular age-related macular degeneration. Cost Eff Resour Alloc 2008;6:12.

51. Stein JD, Newman-Casey PA, Mrinalini T, Lee PP, Hutton DW. Cost-effectiveness of bevacizumab and ranibizumab for newly diagnosed neovascular macular degeneration. Ophthalmology 2014;121:936945 .

52. Brown MM, Brown GC, Sharma S, Landy J. Health care economic analyses and value-based medicine. Surv Ophthalmol 2003;48:204-223.

53. Gower EW, Cassard SD, Bass EB, Schein OD, Bressler NM. A cost-effectiveness analysis of three treatments for age-related macular degeneration. Retina 2010;30:212-221.

54. Brown MM, Brown GC, Sharma S, Landy J, Bakal J. Quality of life with visual acuity loss from diabetic retinopathy and age-related macular degeneration. Arch Ophthalmol 2002;120:481-484. 
55. Brown MM, Brown GC, Brown HC, Peet J. A value-based medicine analysis of ranibizumab for the treatment of subfoveal neovascular macular degeneration. Ophthalmology 2008;115:1039-1045 elo35.

56. Meads C, Moore D. The clinical effectiveness and cost utility of photodynamic therapy for agerelated macular degeneration (Structured abstract). Birmingham: West Midlands Health Technology Assessment Collaboration 2001;48.

57. Athanasakis K, Fragoulakis V, Tsiantou V, Masaoutis P, Maniadakis N, Kyriopoulos J. Costeffectiveness analysis of ranibizumab versus verteporfin photodynamic therapy, pegaptanib sodium, and best supportive care for the treatment of age-related macular degeneration in Greece. Clin Ther 2012;34:446-456.

58. Brown GC, Brown MM, Brown HC, Kindermann S, Sharma S. A Value-Based Medicine Comparison of Interventions for Subfoveal Neovascular Macular Degeneration. Ophthalmology 2007;114:1170-1178.

59. Neubauer AS, Holz FG, Schrader W, et al. [Cost-utility analysis of ranibizumab (Lucentis) in neovascular macular degeneration]. Klin Monatsbl Augenheilkd 2007;224:727-732.

6o. Bansback N, Czoski-Murray C, Carlton J, et al. Determinants of health related quality of life and health state utility in patients with age related macular degeneration: the association of contrast sensitivity and visual acuity. Qual Life Res 2007;16:533-543.

61. Smiddy WE. Economic implications of current age-related macular degeneration treatments. Ophthalmology 2009;116:481-487.

62. Raftery J, Clegg A, Jones J, Tan SC, Lotery A. Ranibizumab (Lucentis) versus bevacizumab (Avastin): modelling cost effectiveness. Br J Ophthalmol 2007;91:1244-1246.

63. Bansback N, Davis S, Brazier J. Using contrast sensitivity to estimate the cost-effectiveness of verteporfin in patients with predominantly classic age-related macular degeneration (Provisional abstract). Eye 2007;21:1455-1463.

64. Fletcher EC, Lade RJ, Adewoyin T, Chong NV. Computerized model of cost-utility analysis for treatment of age-related macular degeneration. Ophthalmology 2008;115:2192-2198.

65. Smiddy WE. Relative cost of a line of vision in age-related macular degeneration. Ophthalmology 2007;114:847-854.

66. Cohen SY, Bremond-Gignac D, Quentel G, et al. Cost-effectiveness sequential modeling of ranibizumab versus usual care in age-related macular degeneration. Graefes Arch Clin Exp Ophthalmol 2008;246:1527-1534.

67. Greiner RA. Cost of care for patients with age-related macular degeneration in Switzerland and cost-effectiveness of treatment with verteporfin therapy. Semin Ophthalmol 2001;16:218-222.

68. Hopley C, Salkeld G, Mitchell P. Cost utility of photodynamic therapy for predominantly classic neovascular age related macular degeneration. Br J Ophthalmol 2004;88:982-987.

69. Sharma S, Bakal J, Sharma SM, Covert D, Shah GK. Drug pricing for a novel treatment for wet macular degeneration: using incremental cost-effectiveness ratios to ensure societal value. Can J Ophthalmol 2005;40:369-377.

70. Donati G. [Cost-effectiveness of photodynamic therapy with verteporfin for choroidal neovascularization in age-related macular degeneration in routine clinical practice in Switzerland]. J Fr Ophtalmol 2007;30:837-841.

71. Muslera E, Natal C. [Cost-effectiveness of photodynamic therapy in age-related macular degeneration]. Arch Soc Esp Oftalmol 2006;81:199-204.

72. Tufail A, Xing W, Johnston R, et al. The neovascular age-related macular degeneration database: multicenter study of 92976 ranibizumab injections: report 1: visual acuity. Ophthalmology 2014;121:1092-1101. 
73. Rosenfeld PJ, Brown DM, Heier JS, et al. Ranibizumab for neovascular age-related macular degeneration. N Engl J Med 2006;355:1419-1431.

74. Berg K, Pedersen TR, Sandvik L, Bragadottir R. Comparison of ranibizumab and bevacizumab for neovascular age-related macular degeneration according to LUCAS treat-and-extend protocol. Ophthalmology 2015;122:146-152. 
4.
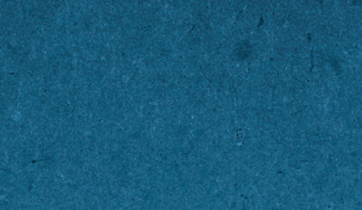

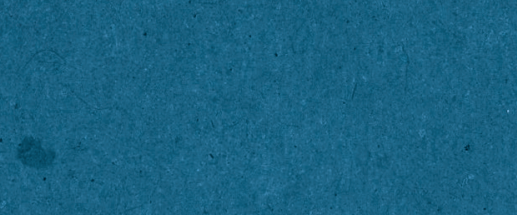
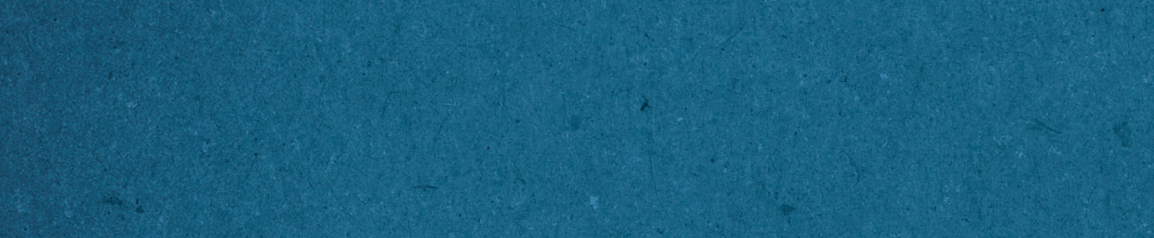

8

$y 3$

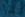




\section{Chapter 4}

The cost-utility of aflibercept for the treatment of age-related macular degeneration compared to bevacizumab and ranibizumab and the influence of model parameters

Mari Elshout, Margriet I. van der Reis, Carroll A.B. Webers, Jan S.A.G. Schouten 


\section{Abstract}

Background: Neovascular age-related macular degeneration (nAMD) is a blinding disease placing considerable burden on society due to blindness-associated costs. Intravitreal anti-vascular endothelial growth factors (anti-VEGFs) are effective in reducing the incidence of blindness, but at potentially high costs, depending on the cost of the drug used. Aflibercept has been introduced as an anti-VEGF equally effective to ranibizumab, but less costly. For this new drug, new cost-effectiveness analyses are needed, and nAMD models used today give biased results. We investigated the costeffectiveness of aflibercept compared to bevacizumab, ranibizumab, and no treatment and studied the influence of commonly used model parameters.

Methods: A patient-level, visual acuity-based, 2-eye model was developed. Data on effectiveness were derived from randomised controlled trials evaluating the outcomes of aflibercept, bevacizumab, and ranibizumab. Utility and resource utilisation were assessed in interviews with nAMD patients. Costs were based on standard health care cost prices. Time horizons were two and five years. A societal perspective was employed.

Results: Over five years, costs associated with aflibercept treatment were $€ 36,030$, with 2.15 QALYs. Costs associated with the bevacizumab regimens, ABC study as-needed (PRN); CATT study PRN; and CATT study $1 \times /$ month, were $€ 19,367 ; € 26,746$; and $€ 30,520$, with $2.16 ; 2.17$; and 2.15 QALYs, respectively. Costs associated with ranibizumab PRN and $1 \times /$ month were $€ 45,491$ and $€ 74,837$ with 2.16 and 2.15 QALYs, respectively. 'No treatment' was associated with $€ 9530$ and 1.96 QALYs. The incremental cost-effectiveness ratios versus 'no treatment' were: aflibercept- $€ 140,274$; bevacizumab- $€ 51,062$ (ABCPRN), $€ 83,256$ (CATT PRN) and $€ 110,361$ (1×/month); ranibizumab- $€ 181,667$ (PRN) and $€ 349,773$ (1×/month). Results were highly dependent on whether only one or both eyes were included, length of time horizon, and whether the costs of blindness and low-vision were included in the analysis.

Conclusions: Aflibercept is a cost-effective treatment for nAMD over ranibizumab. However, aflibercept is not a cost-effective treatment when compared to bevacizumab. Application of inappropriate model assumptions leads to a biased cost-saving estimate of the cost-effectiveness of aflibercept. Therefore, cost-effectiveness analyses should be conducted with appropriate models. 


\section{Introduction}

Neovascular age-related macular degeneration (nAMD) is the leading cause of blindness among elderly persons in industrialised countries., ${ }^{1,}$ Left untreated, the disease leads to blindness and is associated with considerable social and economic cost. nAMD can be treated with intravitreal anti-vascular endothelial growth factors (anti-VEGFs) and blindness and blindness-associated costs can be prevented. However, the costs of the anti-VEGF ranibizumab are considerable. When compared to bevacizumab it cannot be a cost-effective treatment, since bevacizumab is noninferior in its effect and costs much less.3, ${ }^{4}$ Recently, aflibercept has been registered for the treatment of nAMD. Aflibercept, given every two months, has been shown to be non-inferior to a monthly regimen of ranibizumab. ${ }^{5}$ Its price per vial is comparable to that of ranibizumab, but it is more cost-effective due to the lower frequency of injection required as compared to monthly ranibizumab. New cost-effectiveness analyses are needed to quantify the difference in costs. In particular, analyses are needed that compare aflibercept with ranibizumab as needed (pro re nata, PRN) and with the much cheaper bevacizumab. We conducted an analysis comparing aflibercept with ranibizumab and bevacizumab. Moreover, we conducted an analysis to calculate the theoretical frequency of injection that would make aflibercept cost-effective as compared to bevacizumab PRN.

The current paper comprises a model-based cost-effectiveness analysis, incorporating the results on the effectiveness of aflibercept. Solutions to common shortcomings in the design of current cost-effectiveness analyses on the treatment of nAMD were incorporated in this analysis. First, very few cost-effectiveness studies on nAMD treatments take into account the fact that the second eye is mostly unaffected, betterseeing, and not treated initially.-9 However, quality of life is largely dependent on visual acuity in the better-seeing eye. ${ }^{10}$ Taking into account the second eye implies that initially, treatment of the first eye has marginal influence on vision-related quality of life while high treatment-related expenses are already being made. In modelling an ophthalmic disease, it is more realistic to incorporate both eyes in the analysis. Second, the time-horizon used in most studies is limited to 1 or 2 years, which matches the follow-up of the pivotal nAMD trials, but it is shorter than the timespan patients are usually treated in a clinical setting. It is more appropriate to simulate treatment and disease and the effect on quality of life for longer than 2 years. A longer time horizon is also needed because in a 2-eye model, the difference in utility gain is more pronounced after a longer time, when more second eyes become affected and subsequently treated. Third, the extrapolation of visual acuity beyond 1 or 2 years is done in most studies by the method of last observation carried forward, while no treatment is being given 
in that period. Using this method, visual acuity remains stable in the period after 1 or 2 years, without further treatment. However, it is more realistic to assume that at least some treatment is being given or that visual acuity worsens without further treatment. Fourth, many studies also do not include the cost of blindness or low-vision, such as visual aids or rehabilitation. Current guidelines for cost-effectiveness analyses recommend that these costs be taken into account. 


\section{Material and methods}

We developed a model-based analysis for calculating the cost-effectiveness of aflibercept, bevacizumab, and ranibizumab. In this model, the change in visual acuity is based on major trials published in recent years. Direct costs such as the costs of the treatment and outpatient visits are based on Dutch cost pricing of the treatments. Indirect cost, quality of life and utility assessments are based on a cross-sectional study in 184 Dutch nAMD patients.

\section{The Model}

We developed the computational model using Microsoft Excel. The model comprises a Monte Carlo simulation at patient level. Entities ("patients") are simulated one by one. Table 1 illustrates the sequence of events in the model for a single entity. It shows the change in visual acuity over time in each eye; the diagnostics performed and the injections given. The visual acuity in the best eye defines the utility at any given point in time. We defined a Macro code which repeats the simulation for each of 1000 entities, comprising a virtual population.

\section{Perspective \& Discounting}

The perspective of the current analysis is the societal perspective, including direct costs related to the treatment and the indirect costs, such as additional home care due to the disease. A third party payer perspective is used in univariate sensitivity analysis. The discounting of costs is $4 \%$ per annum, discounting of outcomes $1.5 \%$, in accordance with Dutch standards for cost-effectiveness studies."

\section{Population}

Population characteristics such as baseline visual acuity and age were modelled stochastically. They are, where possible, based on weighted averages of the respective baseline characteristics of the population in the source trials: age of the simulated population was 77.7 (SD 7.8) years, 59.6\% of the population was female and baseline visual acuity in affected eyes was 58.6 (SD 14) ETDRS letters. Visual acuity in non-affected eyes was modelled using a triangular distribution with mode 75; maximum 85; and minimum 50 letters. 
Table 1. Modelled visual acuity and events for one example entity, receiving an aflibercept treatment regimen.

\begin{tabular}{|c|c|c|c|c|c|c|}
\hline \multirow[b]{2}{*}{$\begin{array}{l}\text { Time } \\
\text { (months) }\end{array}$} & \multicolumn{4}{|c|}{ Event } & \multicolumn{2}{|c|}{ Visual Acuity } \\
\hline & $\begin{array}{c}\text { Medical } \\
\text { Visit }\end{array}$ & OCT & FA & Injection & Eye 1 & Eye 2 \\
\hline o & Visit & Eye 1 & Eye 1 & Eye 1 & 49.8 & 66.4 \\
\hline 1.0 & & & & Eye 1 & 52.2 & 66.2 \\
\hline 2.0 & & & & Eye 1 & 54.5 & 66.1 \\
\hline 3.0 & Visit & Eye 1 & & & 56.8 & 65.9 \\
\hline 4.0 & & & & Eye 1 & 56.8 & 65.8 \\
\hline 6.0 & Visit & Eye 1 & & Eye 1 & 56.8 & 65.5 \\
\hline 8.0 & & & & Eye 1 & 56.8 & 65.2 \\
\hline 9.0 & Visit & Eye 1 & & & 56.8 & 65.0 \\
\hline 10.0 & & & & Eye 1 & 56.8 & 64.8 \\
\hline 12.0 & Visit & Eye 1 & & Eye 1 & 56.8 & 64.5 \\
\hline 15.0 & Visit & Eye 1 & & Eye 1 & 56.8 & 64.1 \\
\hline 18.0 & Visit & Eye 1 & & Eye 1 & 56.8 & 63.6 \\
\hline 21.0 & Visit & Eye 1 & & Eye 1 & 56.8 & 63.1 \\
\hline 24.0 & Visit & Eye 1 & & Eye 1 & 56.8 & 62.7 \\
\hline 27.0 & Visit & Eye 1 & & Eye 1 & 56.7 & 62.2 \\
\hline 30.0 & Visit & Eye 1 & & Eye 1 & 56.5 & 61.7 \\
\hline 33.0 & Visit & Eye 1 & & Eye 1 & 56.4 & 61.3 \\
\hline 36.0 & Visit & Eye 1 & & Eye 1 & 56.2 & 60.8 \\
\hline 37.2 & & $n A$ & eye 2 & & 56.2 & 50.8 \\
\hline 37.2 & Visit & Eye 2 & Eye 2 & & 56.2 & 50.8 \\
\hline 37.2 & & & & Eye 2 & 56.2 & 50.8 \\
\hline 38.2 & & & & Eye 2 & 56.1 & 53.1 \\
\hline 39.0 & Visit & Eye 1 & & Eye 1 & 56.1 & 55.0 \\
\hline 39.2 & & & & Eye 2 & 56.1 & 55.4 \\
\hline 40.2 & Visit & Eye 2 & & & 56.0 & 57.8 \\
\hline 41.2 & & & & Eye 2 & 56.0 & 57.8 \\
\hline 42.0 & Visit & Eye 1 & & Eye 1 & 55.9 & 57.8 \\
\hline 43.2 & Visit & Eye 2 & & Eye 2 & 55.9 & 57.8 \\
\hline 45.0 & Visit & Eye 1 & & Eye 1 & 55.8 & 57.8 \\
\hline 45.2 & & & & Eye 2 & 55.8 & 57.8 \\
\hline 46.2 & Visit & Eye 2 & & & 55.7 & 57.8 \\
\hline 47.2 & & & & Eye 2 & 55.7 & 57.8 \\
\hline 48.0 & Visit & Eye 1 & & Eye 1 & 55.6 & 57.8 \\
\hline 49.2 & Visit & Eye 2 & & Eye 2 & 55.6 & 57.8 \\
\hline 51.0 & Visit & Eye 1 & & Eye 1 & 55.5 & 57.8 \\
\hline
\end{tabular}


Table 1. Continued.

\begin{tabular}{|c|c|c|c|c|c|c|}
\hline \multirow[b]{2}{*}{$\begin{array}{l}\text { Time } \\
\text { (months) }\end{array}$} & \multicolumn{4}{|c|}{ Event } & \multicolumn{2}{|c|}{ Visual Acuity } \\
\hline & $\begin{array}{c}\text { Medical } \\
\text { Visit }\end{array}$ & OCT & FA & Injection & Eye 1 & Eye 2 \\
\hline 52.2 & Visit & Eye 2 & & Eye 2 & 55.4 & 57.8 \\
\hline 54.0 & Visit & Eye 1 & & Eye 1 & 55.3 & 57.8 \\
\hline 55.2 & Visit & Eye 2 & & Eye 2 & 55.3 & 57.8 \\
\hline 57.0 & Visit & Eye 1 & & Eye 1 & 55.2 & 57.8 \\
\hline 58.2 & Visit & Eye 2 & & Eye 2 & 55.1 & 57.8 \\
\hline
\end{tabular}

Other characteristics of the population were based on a cross-sectional study of costs and quality of life in 184 Dutch patients with nAMD. Visual acuity in non-affected eyes was based on the assumption that visual acuity was on average 10 letters lower than 1.0 Snellen visual acuity: 75 letters with a SD of 5 letters. Of the 184 nAMD patients who participated in our economic study, $5.4 \%$ of the participants had bilateral disease at baseline. Therefore, 5.4\% of entities in the model have nAMD in both eyes at baseline. The incidence of $\mathrm{nAMD}$ in the other eye is based on a systematic review of studies on the risk of developing $\mathrm{nAMD}$ in the second eye. ${ }^{12}$ The percentage of entities developing nAMD in the second eye over 2 years is $15 \%$; 5 years, 33\%; lifetime, $49 \%$. The life-expectancy of entities was based on the life-expectancy of the Dutch general population. ${ }^{13}$

\section{Costs and utilities study}

In 2010 and 2011, we undertook a cross-sectional study to evaluate indirect costs and utility of Dutch patients with nAMD in the South-Limburg, Eindhoven and Amsterdam areas. 184 patients were included in the study. Two authors (ME and MvdR) visited the patients at home. Visual acuity was measured using Radner reading cards and converted to ETDRS letters. Indirect costs were assessed by applying a standardised questionnaire. We asked the patients which and how many lowvision aids they were using because of nAMD; how many times they received visual rehabilitation contacts for $\mathrm{nAMD}$; how often they received professional or informal home care; and what means of transport they used to visit the ophthalmologist. Results were multiplied by the unit cost of the item or service ${ }^{11,14}$ and stratified by visual acuity. Utility was measured using the Health Utilities Index issue 3 (HUI-3) questionnaire. We performed linear regression analysis with the HUI-3 quality of life score as the dependent, and visual acuity in the best eye of the patients, as the independent variable. The regression coefficient was 0.004 , which implies a 0.004 HUI-3 score decrease in quality of life per letter of visual acuity lost. 


\section{Costs}

The direct medical costs included in the model were (1) costs of the diagnostic process - intake visit; fluorescein angiography (FA); optical coherence tomography (OCT); and fundus photography, (2) costs of the treatments: the costs of the medication; the costs of administering the medication (intravitreal injection), and (3) The costs of follow-up: OCT and follow-up visits. Table 2 lists the direct cost estimates used in the model.

Table 3 and Table 4 show the estimates used for the indirect costs. Indirect costs included were cost of transportation, cost of extra home care due to nAMD, and costs of a nursing home because of nAMD. Also, the cost of low-vision services and aids, and moving house because of nAMD were included. Furthermore, the costs of certain serious adverse events of the treatments were included. All costs in the model were inflated to 2012 euros using the Dutch consumer price index.15 The incremental cost-effectiveness ratios as calculated with the model were converted to values for other countries using Purchasing Power Parities (PPP). ${ }^{16}$

Table 2. Event-related cost estimates used in the model.

\begin{tabular}{lr}
\hline Item / Event & Unit Cost, $€$ \\
\hline Medical visit (intake or follow-up) & 78.40 \\
Fluorescein angiography & 73.54 \\
Optical coherence tomography & 38.96 \\
Fundus photograph & 36.03 \\
Aflibercept, drug cost per injection & 906.88 \\
Ranibizumab, drug cost per injection & 773.24 \\
Bevacizumab, drug cost per injection* & 38.37 \\
Intravitreal injection, excl. drug cost & 321.00 \\
Treatment of endophthalmitis & 3345.10 \\
Treatment of retinal detachment & 2174.56 \\
Treatment of lens injury & 1645.87 \\
Treatment of retinal hemorrhage & 216.60 \\
\hline
\end{tabular}

* Includes pharmacy cost for sterile dispensing.

\section{Utility}

In the model, utility, or quality of life, was calculated from the entities' visual acuity in the best eye using the regression coefficient from the costs and utilities study. Qualityadjusted life-years (QALYs) are calculated from the area-under-the-curve of the utility of the entity multiplied by its duration of existence in the model, which is ended by its death or by the end of the time horizon being reached. 
Table 3. Visual acuity-related costs used in the model.

\begin{tabular}{lcccc}
\hline & \multicolumn{2}{c}{ Cost per month, $€$} & & Cost per visit, $€$ \\
\cline { 2 - 3 } Entities' Visual Acuity in & Professional & Informal Care & & Transportation \\
Best Eye, ETDRS Letters & Home Care & 715 & 370 & 16.34 \\
\hline$<20$ & 190 & 234 & 13.88 \\
$\geq 20$ & 70 & 40 & 13.73 \\
$\geq 35$ & 152 & 63 & 12.19 \\
$\geq 60$ & 70 & 5 & 10.32 \\
$\geq 70$ & & & & \\
\hline
\end{tabular}

Table 4. Visual acuity transition-related costs in the model.

\begin{tabular}{lccc}
\hline \multirow{2}{*}{$\begin{array}{l}\text { Change in Entities' Visual Acuity } \\
\text { Stratum in Best Eye, ETDRS Letters }\end{array}$} & Low-vision Aids & Low-vision Services & Moving House \\
\hline & 817 & 653 & 52 \\
$\geq 20$ to $<20$ & 2412 & 39 & 0 \\
$\geq 35$ to $\geq 20$ & 485 & 204 & 0 \\
$\geq 60$ to $\geq 35$ & 0 & 12 & 21 \\
$\geq 70$ to $\geq 60$ & 0 & 0
\end{tabular}

Costs are accrued when entity's visual acuity in best eye crosses threshold defined in first column.

\section{Treatment effect and treatment schedule}

Treatment effect was based on pivotal trials on the treatment of nAMD.4,5,17,18 The effect was defined as a change in letters of visual acuity per time-unit. This change was modelled stochastically: each entity was assigned from a normal distribution a specific change in visual acuity under treatment. The effectiveness estimates are displayed in Table 5. The treatment schedules based on the respective trials are also displayed in Table 5. Eyes were no longer treated when they worsened to or below 10 letters (1/60 visual acuity).

\section{Sensitivity analysis}

Our univariate sensitivity analyses focuses on the relevance of including one eye or both eyes; assuming the societal perspective or only considering direct costs (third-party payer perspective); assuming a short or a long time horizon. Furthermore, univariate analyses were carried out with altered treatment effect; treatment costs; and utility values. Another sensitivity analysis considered the treatment interval of aflibercept treatment. We studied aflibercept versus bevacizumab and ranibizumab treatment using hypothetical scenarios for the aflibercept regimen. The frequency of administration of aflibercept was decreased in each new scenario. The point at which aflibercept reached equal cost-effectiveness to bevacizumab was investigated. 
Table 5. Treatment effectiveness estimates and treatment schedules in the model, per affected eye.

\begin{tabular}{|c|c|}
\hline \multirow[b]{2}{*}{ Schedule } & Aflibercept \\
\hline & Once per 2 months \\
\hline & VIEW $1 \& 2^{5}$ \\
\hline \multicolumn{2}{|l|}{ Source Trial } \\
\hline \multicolumn{2}{|c|}{ Effect (change in visual acuity, letters / month) } \\
\hline $1^{\text {st }} 3$ months & $2.33 \pm 1.2$ \\
\hline After 3 months & $\mathrm{O} \pm 1.2$ \\
\hline Extrapolation beyond 2 y & -0.05 \\
\hline \multicolumn{2}{|c|}{ Treatment frequency (intravitreal injections) } \\
\hline $1^{\text {st }} 3$ months & 3 \\
\hline After 3 months & 1 per 2 months in y 1 ; avg. of 3.6 in y 2 \\
\hline Extrapolation beyond 2 y & Avg: 3.6 per $y$ \\
\hline \multicolumn{2}{|l|}{ Medical visits, with OCT } \\
\hline Treatment start & $1(+\mathrm{FA})$ \\
\hline After 3 months & 1 per 3 months \\
\hline
\end{tabular}

Avg, average; FA, fluorescein angiography; OCT, optical coherence tomography; $P R N$, treatment as needed.

In a multivariate analysis, we set out to investigate the importance of factors combined in influencing the outcome, using two different sets of assumptions. The first set of assumptions is one with which it is less likely that aflibercept will be cost-effective: A short time horizon of 2 years; both eyes included in the analysis; and the costs of blindness and low-vision not included. The second set of assumptions is one with which it is more likely that aflibercept will be cost-effective: Patients treated for 2 years with aflibercept, after which the visual acuity was extrapolated using last observation carried forward, without any treatment being given, up to a 10-year time-horizon; only the affected eye included in the analysis; and the costs of blindness included. 


\begin{tabular}{ccc}
\multicolumn{3}{c}{ Bevacizumab } \\
\hline $\mathrm{PRN}$ & $\mathrm{PRN}^{2}$ & $1 \mathrm{x} /$ month \\
\hline $\mathrm{ABC}^{18}$ & CATT $^{4}$ & CATT
\end{tabular}

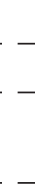

\begin{tabular}{|c|c|c|}
\hline \multicolumn{2}{|c|}{ Ranibizumab } & No Treatment \\
\hline PRN & $1 \mathrm{x} / \mathrm{month}$ & - \\
\hline CATT & MARINA $^{17}$ & $\begin{array}{l}\text { Review of } \\
\text { literature }\end{array}$ \\
\hline
\end{tabular}

$\begin{array}{lccccc}(18 \text { weeks }) & 2.03 \pm 1.3 & 1.7 \pm 1.3 & 1.9 \pm 0.7 & 1.97 \pm 0.7 & -1.34 \\ 1.498 \pm 1.3 & & & & \\ 0.004 \pm 1.3 & 0.21 \pm 1.3 & 0.03 \pm 1.3 & 0.13 \pm 0.7 & 0.033 \pm 0.7 & -1.17\end{array}$

$-0.05$

$-0.05$

$-0.05$

$-0.05$

$-0.05$

$-0.5$

3 in 18 weeks

3

3

3

3

O

Avg: 4.1, min Avg: 4.7 per $9 \quad 1$ per month

3.9 per $9 \quad 1$ per month

O

o; $\max 6$ per 9 months

months

months

Avg: 4.1, min

4.7 per 9

1 per month

3.9 per 9

1 per month

$\mathrm{O}$

o; max 6 per 9

months

months

months

\begin{tabular}{|c|c|c|c|c|c|}
\hline $1(+\mathrm{FA})$ & $1(+\mathrm{FA})$ & $1(+\mathrm{FA})$ & $1(+\mathrm{FA})$ & $1(+\mathrm{FA})$ & $1(+\mathrm{FA})$ \\
\hline 1 per 6 weeks & 1 per month & $\begin{array}{l}1 \text { per } 3 \\
\text { months }\end{array}$ & 1 per month & $\begin{array}{l}1 \text { per } 3 \\
\text { months }\end{array}$ & 1 per year \\
\hline
\end{tabular}

Further, we enabled the model for probabilistic analysis. To this end, after the complete population has been simulated, new values are drawn from pre-defined distributions for appropriate probabilistic parameters and the entire population was re-simulated. This probabilistic simulation was repeated for 1000 times. The distributions of the probabilistic parameters are defined in Table 6. 
Table 6. Model input: probabilistic parameters and their distributions.

\begin{tabular}{|c|c|c|c|c|}
\hline \multirow[b]{2}{*}{ Model Parameter } & \multirow[b]{2}{*}{ Base-case Value } & \multicolumn{2}{|r|}{ Distribution } & \multirow[b]{2}{*}{ Source } \\
\hline & & Type & Parameters & \\
\hline \multicolumn{5}{|c|}{ Adverse events, risk per injection of ranibizumab or aflibercept } \\
\hline Endophthalmitis & 0.0004 & Beta & Alpha: 3.8; beta: 9595 & 19 \\
\hline Retinal detachment & 0.0001 & Beta & Alpha: 0.4 ; beta: 4267 & 19 \\
\hline Lens injury & 0.0001 & Beta & Alpha: 1.o; beta: 9601 & 19 \\
\hline Retinal hemmorrhage & 0.0003 & Beta & Alpha: 3.8; beta: 12797 & 19 \\
\hline \multicolumn{5}{|c|}{ Adverse events, risk per injection of bevacizumab } \\
\hline Endophthalmitis & 0.0005 & Beta & Alpha: 3.8 ; beta: 7645 & 19 \\
\hline Retinal detachment & 0.0007 & Beta & Alpha: 7.5; beta: 10740 & 19 \\
\hline Lens injury & 0.0001 & Beta & Alpha: 1.o; beta: 9601 & 19 \\
\hline Retinal hemmorrhage & 0.0010 & Beta & Alpha: 6.o; beta: 5990 & 19 \\
\hline \multicolumn{5}{|c|}{ Effect of treatment, variation (letters per month) } \\
\hline Aflibercept & O & Normal & Mean: o; SD: 0.0478 & 5 \\
\hline Bevacizumab & $\mathrm{O}$ & Normal & Mean: O; SD: 0.1 & \\
\hline Ranibizumab & o & Triangular & Mode: o; min: -0.06; max: 0.06 & 17 \\
\hline \multicolumn{5}{|c|}{ Costs, multiplication factors } \\
\hline Professional care & 1 & Triangular & Mode: 1; min: 0.15; max: 2 & 11,14 \\
\hline Informal care & 1 & Triangular & Mode: 1; min: 0.3; max: 1.8 & 11,14 \\
\hline Moving house & 1 & Triangular & Mode: 1; min: o; max: 2 & ${ }^{11,14}$ \\
\hline Low-vision services & 1 & Triangular & Mode: 1; min: 0.5; max: 1.5 & 11,14 \\
\hline Low-vision aids & 1 & Triangular & Mode: 1; min: 0.5; max: 1.5 & 11,14 \\
\hline Patient transport & 1 & Triangular & Mode: 1 ; min: 0.5; max: 2 & 11,14 \\
\hline \multicolumn{5}{|l|}{ Utility } \\
\hline $\begin{array}{l}\text { Beta for QoL loss per } \\
\text { letter of VA loss }\end{array}$ & 0.004 & Normal & Mean: 0.004; SD: 0.001 & $\begin{array}{l}\text { Patients, } \\
\text { HUI-3* }^{*}\end{array}$ \\
\hline \multicolumn{5}{|l|}{ Disease progression } \\
\hline $\begin{array}{l}\text { Hazard for developing } \\
\text { nAMD in } 2^{\text {nd }} \text { eye over } \\
36 \text { months }\end{array}$ & 0.212 & Normal & Mean: 0.212; SD: 0.06 & 12 \\
\hline
\end{tabular}

nAMD, neovascular age-related macular degeneration; $H U I-3$, health utilities index mark 3; SD, standard deviation; QoL, quality of life; VA, visual acuity.

* 184 nAMD patients (HUI-3), see text. 


\section{Results}

\section{Primary results}

A cost-effectiveness study allows the various regimens to be compared in terms of their effect on quality of life, as well as in terms of their associated costs. Table 7 shows the results in QALYs and costs for each treatment, for both the 2 and 5 year time-horizon. The incremental cost-effectiveness ratio (ICER) of each treatment versus no treatment is displayed in Table 8. To extend applicability of the results, we converted the ICERs to other currencies using PPPs which is also shown in Table 8. Aflibercept is about equally effective as ranibizumab PRN, but is $€ 9,461$ per patient less expensive over 5 years. Aflibercept is equally effective as bevacizumab PRN (ABC), but it is $€ 16,663$ per patient more expensive over 5 years.

Table 7. Results of cost-effectiveness analysis for treatments for age-related macular degeneration.

\begin{tabular}{|c|c|c|c|c|c|c|}
\hline \multirow[b]{2}{*}{ Treatment } & \multirow[b]{2}{*}{ Schedule } & \multirow[b]{2}{*}{ Study } & \multicolumn{2}{|c|}{2 years } & \multicolumn{2}{|c|}{5 years } \\
\hline & & & QALYs & Cost, $€$ & QALYs & Cost, $€$ \\
\hline Aflibercept & $1 \times / 2$ months & VIEW $1 \& 2$ & 1.02 & 17,963 & 2.15 & 36,030 \\
\hline \multirow[t]{3}{*}{ Bevacizumab } & PRN & $\mathrm{ABC}$ & 1.01 & 8427 & 2.16 & 19,367 \\
\hline & & CATT & 1.02 & 12,664 & 2.17 & 26,746 \\
\hline & $1 \times /$ month & CATT & 1.01 & 13,021 & 2.15 & 30,520 \\
\hline \multirow[t]{2}{*}{ Ranibizumab } & PRN & CATT & 1.01 & 19,919 & 2.16 & 45,491 \\
\hline & $1 \times /$ month & MARINA & 1.01 & 31,706 & 2.15 & 74,837 \\
\hline No treatment & - & Review of literature & 0.96 & 3298 & 1.96 & 9530 \\
\hline
\end{tabular}

$P R N$, pro re nata (as needed).

Table 8. Results of cost-effectiveness analysis, calculations for other countries.

\begin{tabular}{lcccccccccc}
\hline & \multicolumn{7}{c}{ Cost per QALY over No Treatment } \\
\cline { 2 - 10 } Treatment & $\begin{array}{c}\text { Netherlands } \\
\text { (this study) }\end{array}$ & France & Germany & Kingdom & States \\
\hline Aflibercept & $€$ & 140,274 & $€$ & 141,634 & $€$ & 131,943 & $£$ & 117,320 & $\$$ & 179,029 \\
Bevacizumab PRN, ABC & $€$ & 51,062 & $€$ & 52,052 & $€$ & 48,029 & $£$ & 42,706 & $\$$ & 61,893 \\
Bevacizumab PRN, CATT & $€$ & 83,256 & $€$ & 84,870 & $€$ & 78,311 & $£$ & 69,632 & $\$$ & 100,916 \\
Bevacizumab, CATT & $€$ & 110,361 & $€$ & 112,502 & $€$ & 103,807 & $£$ & 92,302 & $\$$ & 133,771 \\
Ranibizumab PRN & $€$ & 181,667 & $€$ & 185,190 & $€$ & 170,877 & $£$ & 151,940 & $\$$ & 220,203 \\
Ranibizumab & $€$ & 349,773 & $€$ & 356,556 & $€$ & 328,998 & $£$ & 292,537 & $\$$ & 423,967 \\
\hline
\end{tabular}




\section{Sensitivity analysis}

We investigated with univariate sensitivity analyses the importance of including one or both eyes in the model, assuming the societal perspective or including only direct costs, and the length of the time horizon, among other parameters, see the tornado plot in Figure 1. Aflibercept is being marketed as a more cost-effective treatment over ranibizumab due to the lower frequency of injections required. We investigated how long the interval between aflibercept injections should be for it to be equally as costeffective as bevacizumab PRN or ranibizumab PRN treatment. Assuming both treatments have the same effect on visual acuity and thus quality of life, we compared the cost of aflibercept treatment with various hypothetical intervals between injections, with the costs of bevacizumab and ranibizumab treatment. Figure 2 shows the results of this sensitivity analysis. It shows that at base case (8-week interval), the costs of aflibercept treatment are lower than ranibizumab PRN. At intervals of 15; 19 and 38 weeks, the cost of aflibercept treatment is comparable to bevacizumab $1 \times /$ month, bevacizumab PRN as in the CATT study; and bevacizumab PRN as in the ABC study, respectively.

In a multivariate sensitivity analysis, we compared the cost per QALY of aflibercept versus no treatment with two different sets of three assumptions that either make it (1) more likely or (2) less likely that aflibercept will be cost-effective: (1) extrapolating visual acuity with last-observation carried forward and a 10-year time-horizon versus (2) a time horizon of 2 years; (1) only the affected eye, versus (2) both eyes included in the analysis; (1) including versus (2) excluding the cost of blindness and low-vision. In the first analysis, aflibercept was dominant over no treatment, meaning that in the aflibercept scenario more QALYs were gained and total costs were lower: the cost-effectiveness ratio was $€-4600 / Q A L Y$. In the second analysis, the cost-effectiveness ratio of aflibercept over no treatment was $€ 276,783 /$ QALY. In comparison, in the analysis with the base-case assumptions, the cost-effectiveness ratio was $€ 140,274 /$ QALY.

Figure 3 displays the cost-effectiveness acceptability curve (CEAC) for the treatment regimens over a 5-year time-horizon. It shows the probability of the treatment being costeffective at various levels of willingness to pay ( $€ /$ QLLY). In this analysis, at a willingness to pay of $€$ o to $€ 44,000$ per QALY, not treating is associated with the highest probability of being cost-effective. At a willingness to pay over $€$ 44,000, Bevacizumab PRN (ABC trial) has the highest probability of being cost-effective. At a willingness to pay of over $€$ 343,000, bevacizumab PRN (CATT study) becomes the treatment of choice. Other treatment regimens, aflibercept and ranibizumab (PRN and $1 \times /$ month), do not show a higher probability of being cost effective than either 'no treatment' or bevacizumab PRN (ABC or CATT study) in the willingness-to-pay range of $€$ o to $€ 400,000$. Figure 4 shows the scatterplot of 1,000 probabilistic simulations for each treatment. 


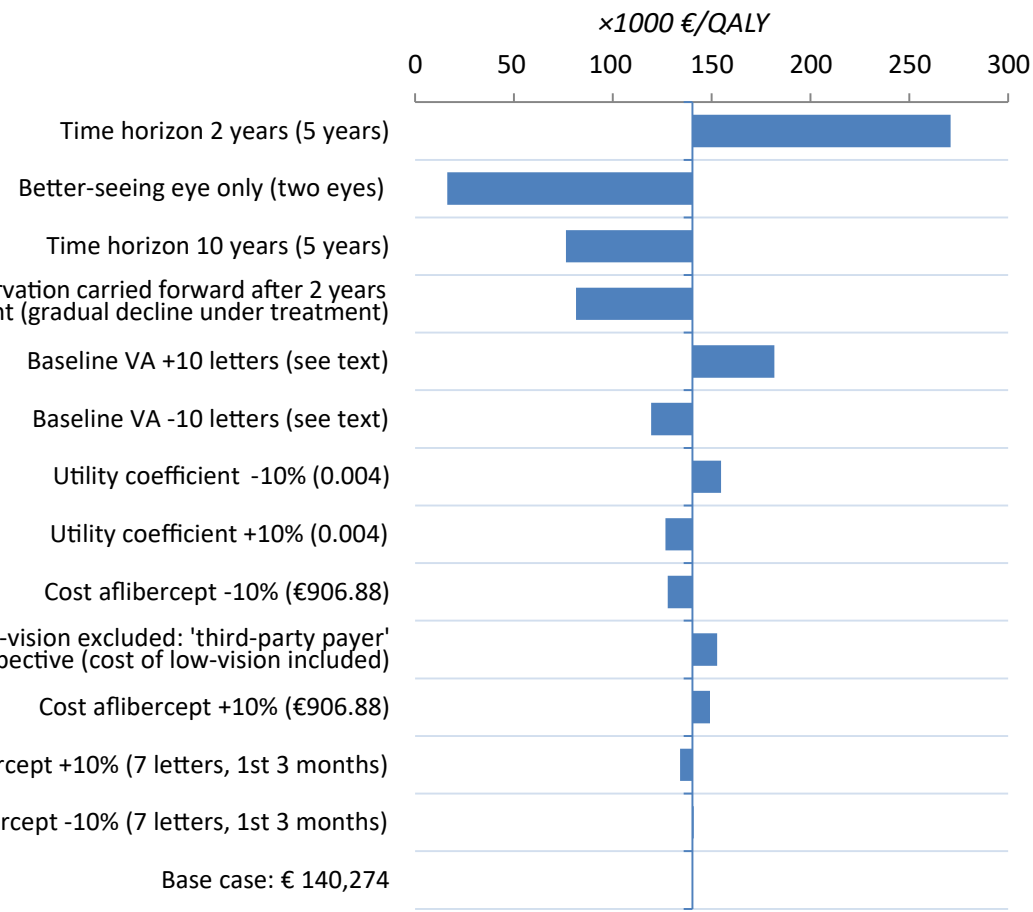

Figure 1. Tornado plot of univariate sensitivity analysis: aflibercept versus no treatment. Base case assumptions in parentheses.

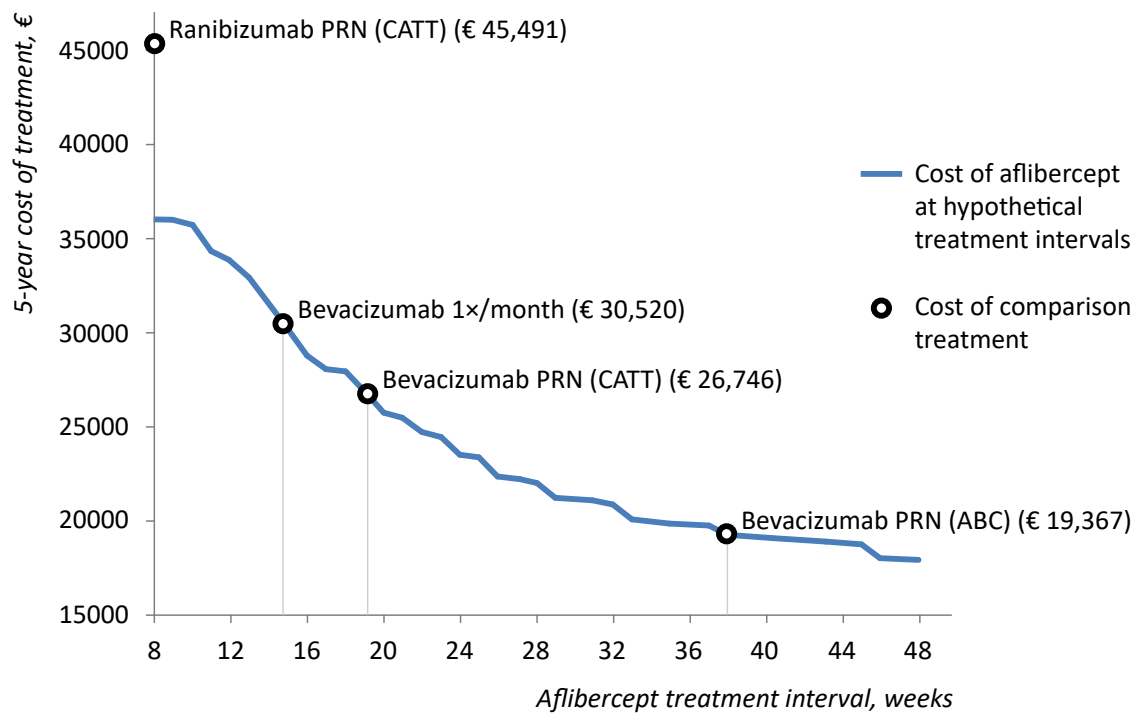

Figure 2.The five-year cost of aflibercept treatment assuming extended intervals between injections, compared to the base-case costs of bevacizumab and ranibizumab treatments. 


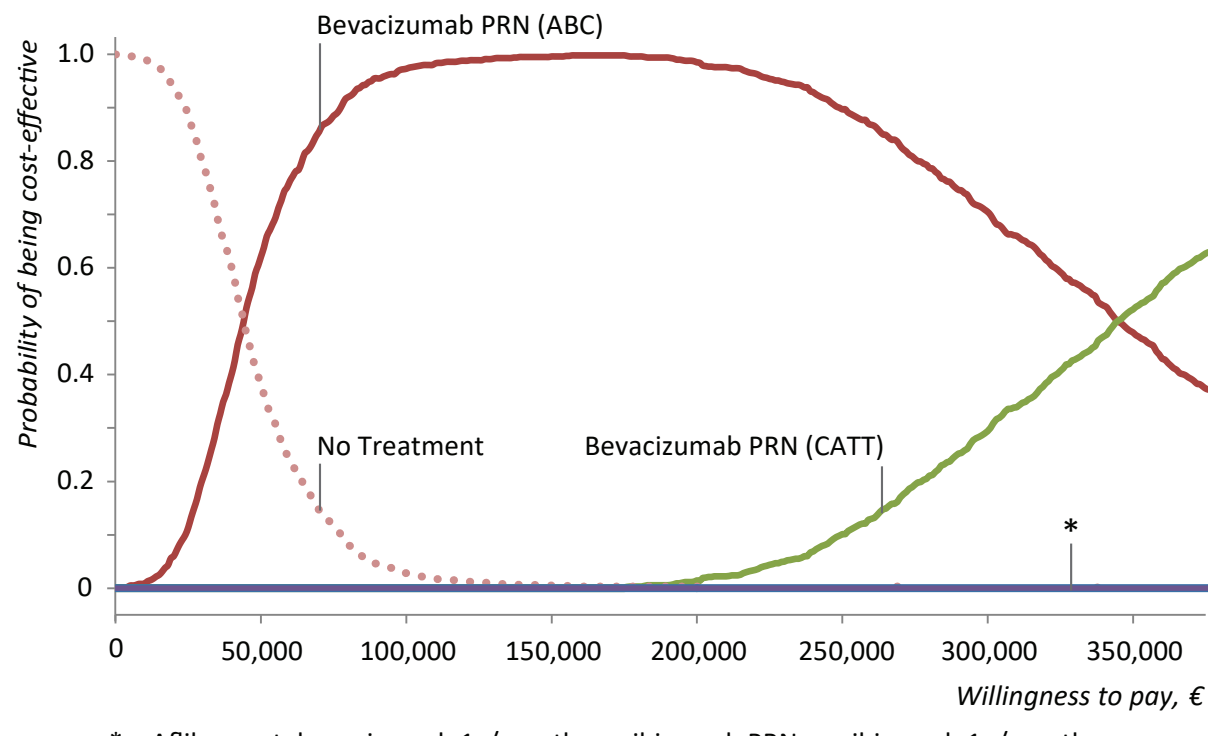

* = Aflibercept; bevacizumab 1x/month; ranibizumab PRN; ranibizumab $1 \times$ /month.

Figure 3. Cost-effectiveness acceptability curve of various treatment regimens for neovascular agerelated macular degeneration, 5-year time-horizon.

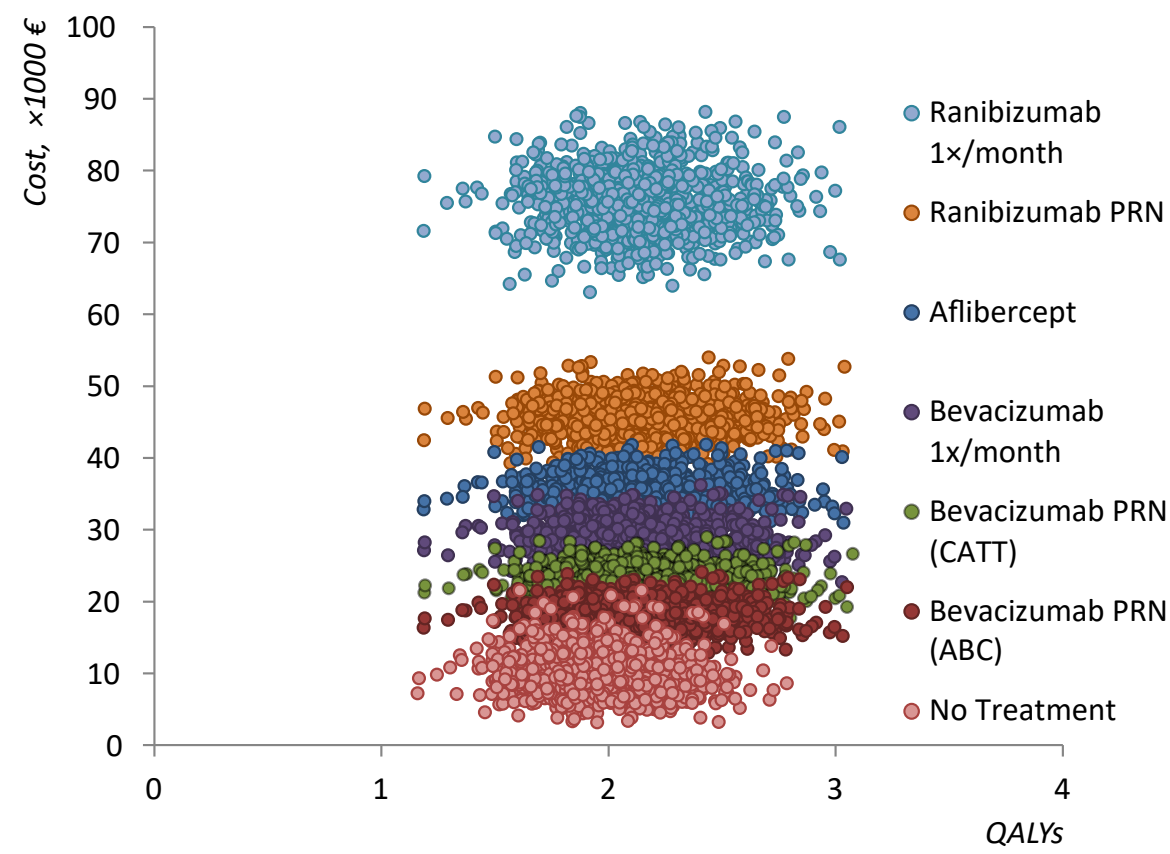

Figure 4. Scatterplot of cost-effectiveness data from probabilistic analysis of treatments for age-related macular degeneration. 


\section{Discussion}

This cost-effectiveness model for nAMD was developed according to existing guidelines for the development of cost-effectiveness models. It contains major improvements as compared to existing cost-effectiveness models in $\mathrm{nAMD}$ which produce biased results. We have shown that the results are highly dependent on several assumptions: including the affected eye only or including both eyes; the length and type of extrapolation; and the in- or exclusion of the cost of blindness. Changing these assumptions can have a dramatic effect on the cost-effectiveness ratio of a treatment. One can assume a set of assumptions that make aflibercept dominant (cost saving and more effective) over 'no treatment'; or assume another set of assumptions that make aflibercept not cost-effective over 'no treatment'. It is important to make realistic assumptions in order to gain realistic results. In our analysis, both eyes are included in the model with an estimate of the incidence of nAMD in the second eye based on a systematic review. The time horizon was extended. There was a more realistic extrapolation of treatment regimens and related change in visual acuity beyond the time horizon of 2 years than the assumption in other studies of a sustained level of visual acuity when no further treatment is given. Cost of blindness and a validated measure of utility were included from a societal perspective. A limitation of this study is that clinical trial data has been used as input for the model. As clinical trial is not similar to clinical reality, this affects external validity of the results.

The model was used to investigate the claim that aflibercept is a cost-effective treatment for nAMD or at least more cost-effective as compared to ranibizumab based on the data of the VIEW-study. ${ }^{5}$ Comparisons were also made with bevacizumab and no treatment and different treatment frequencies were evaluated. The effect of the treatments in terms of quality of life did not differ significantly in the analyses. Obviously, 'no treatment' confers the lowest amount of QALYs. Greater differences exist in the associated costs. The strategy of "no treatment" is associated with the lowest costs, which is the result of no costs for treatment. Aflibercept, marketed as a more cost-effective treatment for nAMD due to the lower frequency of treatments required, is associated with costs lower than a ranibizumab "as-needed" schedule.

Comparing the cost of aflibercept treatment to that of bevacizumab as-needed, it becomes evident that the implied cost savings do not weigh up to the low price of bevacizumab intravitreal injections. Although a frequency of injection of once per 8 weeks is a step forward compared to the standard dosing of once in 4 weeks for ranibizumab treatment, at the current pricing, it would require an interval of 15 to 38 weeks between injections for the costs of aflibercept treatment to rival those of bevacizumab. While the evidence on efficacy and safety of bevacizumab has expanded, it is important to note that its 
use for the treatment of nAMD is off-label. This should be considered together with its outstanding cost-effectiveness over ranibizumab and aflibercept, both of which are approved by the European Medicines Agency for the treatment of nAMD.

\section{Acknowledgments}

This study was supported by the Dutch organisation for health research and development ZonMw, The Hague, The Netherlands, grant number 152001002. 


\section{References}

1. Klaver CC, Wolfs RC, Vingerling JR, Hofman A, de Jong PT. Age-specific prevalence and causes of blindness and visual impairment in an older population: the Rotterdam Study. Arch Ophthalmol 1998;116:653-658.

2. WangJJ, Foran S, Mitchell P. Age-specific prevalence and causes of bilateral and unilateral visual impairment in older Australians: the Blue Mountains Eye Study. Clin Experiment Ophthalmol 2000;28:268-273.

3. Chakravarthy U, Harding SP, Rogers CA, et al. Ranibizumab versus bevacizumab to treat neovascular age-related macular degeneration: One-year findings from the IVAN randomized trial. Ophthalmology 2012;119:1399-1411.

4. Martin DF, Maguire MG, Fine SL, et al. Ranibizumab and bevacizumab for treatment of neovascular agerelated macular degeneration: two-year results. Ophthalmology 2012;119:1388-1398.

5. Heier JS, Brown DM, Chong V, et al. Intravitreal aflibercept (VEGF trap-eye) in wet age-related macular degeneration. Ophthalmology 2012;119:2537-2548.

6. Busbee BG, Brown MM, Brown GC, Sharma S. CME review: A cost-utility analysis of laser photocoagulation for extrafoveal choroidal neovascularization. Retina 2003;23:279-287; ; quiz 443-274.

7. Fletcher EC, Lade RJ, Adewoyin T, Chong NV. Computerized model of cost-utility analysis for treatment of age-related macular degeneration. Ophthalmology 2008;115:2192-2198.

8. Larouche K, Rochon S. Evaluation of photodynamic therapy for the treatment of exudative age-related macular degeneration (ARMD) with subfoveal neovascularization (Structured abstract). Montreal: Agence d'Evaluation des Technologies et des Modes d'Intervention en Sante (AETMIS) 2004;101.

9. Brown MM, Brown GC, Brown HC, Peet J. A value-based medicine analysis of ranibizumab for the treatment of subfoveal neovascular macular degeneration. Ophthalmology 2008;115:1039-1045 e1035.

10. Brown GC. Vision and quality-of-life. Trans Am ophthalmol soc 1999;97:473-511.

11. Oostenbrink JB, Bouwmans CAM, Koopmanschap MA, Rutten FFH. Handleiding voor kostenonderzoek. Methoden en standaard kostprijzen voor economische evaluaties in de gezondheidszorg. Diemen: CVZ; 2004 .

12. Wong TY, Chakravarthy U, Klein R, et al. The natural history and prognosis of neovascular age-related macular degeneration: a systematic review of the literature and meta-analysis. Ophthalmology 2008;115:116126.

13. Generatie-overlevingstafels; geslacht en leeftijd (peilmoment 2008). Gewijzigd op 15 februari 2010. Centraal Bureau voor de Statistiek 2010; [http://statline.cbs.nl/StatWeb/dome/default.aspx] Accessed: 7-6-2010.

14. Roijen LH-v, Tan SS, Bouwmans CAM. Handleiding voor Kostenonderzoek - Methoden en standaard kostprijzen voor economische evaluaties in de gezonheidszorg. Diemen: College voor Zorgverzekeringen; 2010.

15. Statistics Netherlands. Consumer prices; price index $2006=100$. http://statline.cbs.nl.

16. PPPs and Exchange rates. Organisation for Economic Co-operation and Development. http://stats.oecd. org/Index.aspx?datasetcode=SNA_TABLE4 [accessed 9 February 2014].

17. Rosenfeld PJ, Brown DM, Heier JS, et al. Ranibizumab for neovascular age-related macular degeneration. N Engl J Med 2006;355:1419-1431.

18. Tufail A, Patel PJ, Egan C, et al. Bevacizumab for neovascular age related macular degeneration (ABC Trial): multicentre randomised double masked study. Bmj 2010;340:c2459.

19. Van der Reis MI, La Heij EC, Jong-Hesse YD, Ringens PJ, Hendrikse F, Schouten JS. A systematic review of the adverse events of intravitreal anti-vascular endothelial growth factor injections. Retina 2011;31:1449-69. 
4.
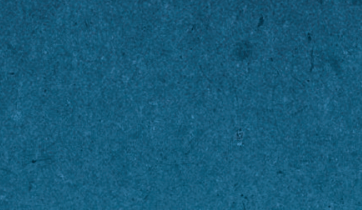

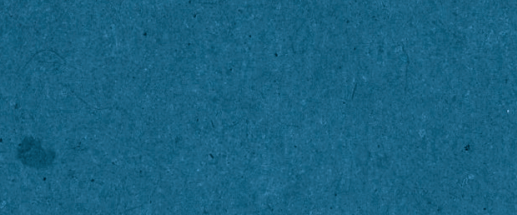
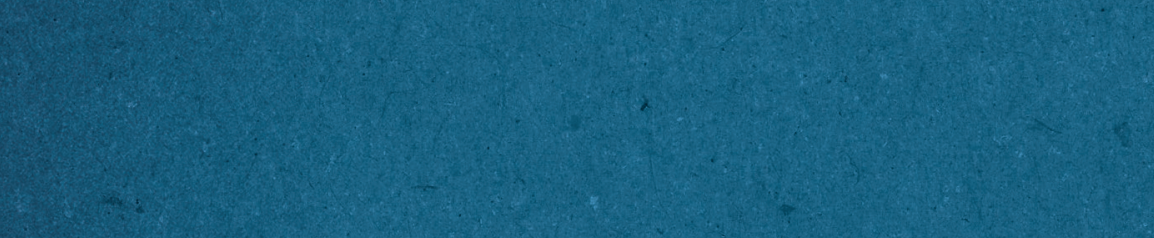

8

$y 3$

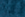




\section{Chapter 5}

\section{A New Epidemiological Aid in Deciding Whether to Continue or Stop a Treatment}

Mari Elshout, Margriet I. van der Reis, Carroll A.B. Webers, Ellen C. La Heij, Fred Hendrikse, Jan S.A.G. Schouten 


\section{Abstract}

Purpose: To present a new epidemiological method relying on randomised controlled clinical trial (RCT) data to assess whether a treatment was effective, aiding in the decision to continue or stop the treatment in clinical patients.

Methods: A cutoff point is calculated in the change of a continuous outcome for which a proportion of treated patients clearly achieved a change better than this cutoff point as a result of the treatment. This cutoff point can then be applied to individual patients during routine therapy. The method was applied to reports of the Minimally Classic/Occult Trial of the Anti-VEGF Antibody Ranibizumab in the Treatment of Neovascular AMD (MARINA) trial, which included patients with nAMD treated with monthly intravitreal injections of ranibizumab, and to reports of trials involving patients with high intra-ocular pressure (IOP), macular edema, and convergence insufficiency.

Results: The cutoff point in the change in visual acuity (number of letters), above which a proportion of patients clearly benefited due to ranibizumab treatment, was -5.0 at 24 months follow-up. The proportion of treated patients who ended above this cutoff point due to the treatment was $60 \%$. The cutoff point varies with time of follow-up and by subgroup.

Conclusions: Contrary to common interpretation, no change, or a limited decline, in the outcome (visual acuity) can still imply that the patients are better off with the treatment than with no treatment. Stopping the treatment above the cutoff point may not be appropriate since it was effective in at least a proportion of patients. This method applies to a broad range of scales and conditions. 


\section{Introduction}

In clinical practice, the decision to continue, discontinue, or change a treatment is a daily problem. In many cases this decision depends strongly on the interpretation of the change from baseline in an outcome such as visual acuity or intra-ocular pressure (IOP).

Rational decision making implies that when the change in outcome indicates that the treatment has been effective, continuing the treatment is a logical decision. Similarly, discontinuing the treatment is appropriate when the treatment has not been effective.

However, it is often difficult to decide whether a treatment has been effective, since the outcome of the natural course of the disease, had the treatment not been given, is not known for treated patients. It is attractive to follow one's intuition and to assume that no change in the outcome, or worsening of the outcome relative to baseline, is proof of no effect. However, this assumption is not supported by evidence.

In this article we present a method to calculate the cutoff point for the change in a continuous outcome variable above which the treatment was clearly effective in a proportion of patients. As an example, we use data from a randomised controlled clinical trial (RCT) of intravitreal ranibizumab versus sham intravitreal injections in neovascular age-related macular degeneration (nAMD). ${ }^{1}$ AMMD is the leading cause of blindness in elderly people in developed countries. ${ }^{2}$ The disease plays a major role in the daily practice of many ophthalmologists. As an important measure of change in disease intensity, the level of change in visual acuity is the continuous outcome variable in nAMD. In addition to this nAMD example, we apply the method to other scales and conditions: Intra-ocular pressure (IOP) reduction with topical medication in elevated IOP, visual acuity in the treatment of refractory diabetic macular edema, and near point of convergence (NPC) in the treatment of convergence insufficiency. 


\section{Methods}

We defined normal distributions using results of randomised trials to calculate the cutoff point above which it is certain that a proportion of treated patients ended due to the treatment. Normal distributions commonly apply to values of observations that cluster around a mean.

For four examples of ophthalmologic treatment, we derived from trial reports the mean change in outcome in the treated group and the nontreated reference group $\left(\mu_{t}\right.$ and $\mu_{r^{\prime}}$ respectively) and the standard deviation (SD) of this change $\left(\sigma_{t}\right.$, and $\sigma_{r}$, respectively) (see Tables 1, 2, 3). We converted SDs from standard errors (SE) or $95 \%$ confidence intervals in the trial reports using the guidelines in the Cochrane Handbook for Systematic Reviews of Interventions. ${ }^{3}$

Several observations can be made when plotting the curves of the normal distributions, or probability density functions (see Figure 1). At the intersection of the curves, the probability densities in both the treated group and the nontreated (placebo) group are equal:

$$
\frac{e^{-\left(x-\mu_{t}\right)^{2} /\left(2 \sigma_{t}^{2}\right)}}{\sigma_{t} \sqrt{2 \pi}}-\frac{e^{-\left(x-\mu_{r}\right)^{2} /\left(2 \sigma_{r}{ }^{2}\right)}}{\sigma_{r} \sqrt{2 \pi}}=0
$$

For patients ending at the corresponding change in outcome, $x$, the probability is therefore zero that this change is due to the treatment. This change in outcome can be calculated by solving for $x$ the following quadratic equation which results from equation 1 :

$$
a x^{2}+b x+c=0
$$

Where

$$
\begin{aligned}
& a=\sigma_{r}^{2}-\sigma_{t}^{2} \\
& b=2 \mu_{r} \sigma_{t}^{2}-2 \mu_{t} \sigma_{r}^{2} \\
& c=\mu_{t}^{2} \sigma_{r}^{2}-\mu_{r}^{2} \sigma_{t}^{2}-2 \sigma_{r}^{2} \sigma_{t}^{2} \cdot \ln \left(\frac{\sigma_{r}}{\sigma_{t}}\right)
\end{aligned}
$$

For the first example, we derived data from the Minimally Classic/Occult Trial of the Anti-VEGF Antibody Ranibizumab in the Treatment of Neovascular AMD (MARINA) trial report. In this trial, participants had nAMD with either minimally classic or occult (with 
no classic lesions) choroidal neovascularization (CNV). They were treated with monthly intravitreal injections of ranibizumab or sham injections. We applied the calculations to the change in Early Treatment Diabetic Retinopathy Study (ETDRS) visual acuity. In this example (Figure 1), at intersection $x$, the number of patients who achieved the corresponding change in visual acuity $x$ due to the treatment with ranibizumab is zero. This change in outcome (visual acuity) is our cutoff point of interest. As illustrated in Figure 1 , for a change in visual acuity above cutoff point $x$, for example, "A" or " $\mathrm{B}$," the probability density in the treated group is greater than in the placebo group, that is, there are more treated patients with that change than there are nontreated patients with the same change. The proportion of treated patients who ended above cutoff point $x$ is the area under the distribution of the treatment group results above $x: A_{t^{\prime}}$ which is calculated using the error function (erf):

$$
A_{t}=\frac{1}{2}\left[1+\operatorname{erf}\left(\frac{x-\mu_{t}}{\sigma_{t} \sqrt{2}}\right)\right]
$$

The proportion of nontreated patients who ended above cutoff point $\mathrm{x}$ is the area under the distribution of the placebo group results above $x$ : $A_{r}$, which is calculated using the error function:

$$
A_{r}=\frac{1}{2}\left[1+\operatorname{erf}\left(\frac{x-\mu_{r}}{\sigma_{r} \sqrt{2}}\right)\right]
$$

The treatment-attributed effect (TAE), that is, the proportion of treated patients who ended above the cutoff point due to the treatment, is calculated using $A_{t}$ and $A_{r}$ :

$$
\mathrm{TAE}=\left(A_{t}-A_{r}\right) / A_{t} \cdot 100 \%
$$

For 1, 3, 6, 12, and 24 months of follow-up in the MARINA trial we calculated cutoff point $x$ and TAE in the change in visual acuity. Furthermore, we have applied the method to MARINA subgroup analysis results by Boyer et al. ${ }^{4}$ This allowed us to assess whether the method yields different results when addressing effect modification. The subgroup analyses were based on the 24-month visual acuity results segregated by age, initial visual acuity, CNV lesion size, or CNV lesion type.

The second example was based on results of a trial of bimatoprost versus placebo by DuBiner et al. ${ }^{5}$ We calculated cutoff point $x$ and TAE in the percentage reduction of IOP 
after 29 days for treatment with bimatoprost for patients with elevated IOP. The third example was based on results of a trial of triamcinolone versus placebo by Dehghan et al. ${ }^{6}$ We calculated cutoff point $x$ and TAE in the change in LogMAR visual acuity after 2 months in the treatment of refractory diabetic macular edema. The fourth example was based on results of a trial of office-based vergence/accommodative therapy with home reinforcement versus, among others, office-based placebo therapy with home reinforcement by the Convergence Insufficiency Treatment Trial Study Group? We calculated cutoff point $x$ and TAE in the 12-week reduction in centimeters of the NPC with vergence/accommodative therapy. 


\section{Results}

We explain the method with some example calculations (Figure 1) based on the MARINA trial results at 24 months. First, we consider the modeled patient group " $\mathrm{A}$ " in Figure 1. This group includes all patients who gained 10 to 11 letters of visual acuity. These patients are represented by areas under the curve in both the intervention and the sham treatment group. The shaded area under the curves for the intervention group (the light area plus the dark area) represents the proportion of all treated patients who gained 10 to 11 letters: 2.3\%. The area under the curve in the sham group (the dark shaded area) is the proportion of all sham patients who also gained 10 to 11 letters: $0.85 \%$. The proportion of patients in the treated group gaining 10 to 11 letters who actually gained that visual acuity due to the treatment (the light shaded area) was $(2.3-0.85) / 2.3 * 100 \%=63 \%$. Group " $\mathrm{B}$ " in Figure 1 contains all modeled patients with a more modest gain of 3 to 4 letters visual acuity. If we do the same calculations for this group, the proportion of patients who gained 3 to 4 letters attributable to the treatment is smaller than that in group A: $42 \%$. At the intersection of the curves, cutoff point $x$, this proportion is reduced to zero; in this example, the cutoff point is -5 (point $x$ in Figure 1 ). At this point, the proportion of patients who achieved this change was equal in both the intervention group and the sham group. The proportion of patients in the treatment group who lost 5 letters attributable to the treatment is therefore zero. This value is our cutoff point at 24 months follow-up. As illustrated with groups A and B, for every value of the change in outcome above this cutoff point (less loss or more gain), there are patients who have achieved the change as a result of the treatment. The more a change in outcome lays above the cutoff point, the larger the proportion of treated patients who achieved this change as a result of the treatment. These patients would not have achieved this outcome if the treatment had not been given.

Table 1 shows the results for five times of follow-up in the MARINA trial report. It shows the mean value and SD in the change in visual acuity derived from the trial article. It also displays the corresponding cutoff point $x$. Furthermore, it shows the proportion of treated patients who ended above this point, $A_{t^{\prime}}$ as well as the proportion of treated patients who ended above this point due to the treatment, TAE. Table 2 shows cutoff point $x$ and TAE, over different levels of effect modifiers in the MARINA trial: age, initial visual acuity, lesion size, and lesion type. The means and SDs used in the calculations are specified. 


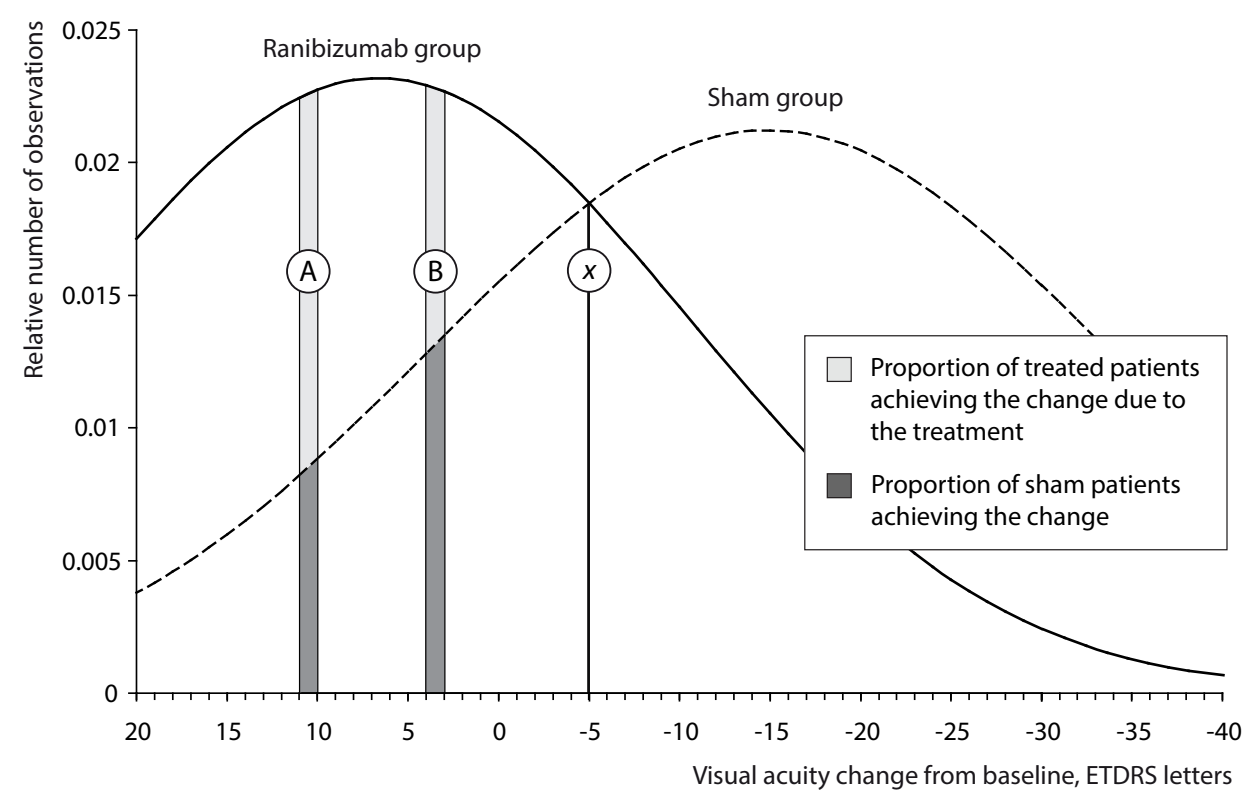

Figure 1. Normal distribution models of change in visual acuity in sham and ranibizumab group at follow-up $=24$ months in the MARINA trial. A: Patients who gained 10 to 11 letters. B: Patients who gained 3 to 4 letters. $x$ : Patients who lost 5 letters.

Table 1. Results for Five Times of Follow-up in the MARINA Trial.

Change in ETDRS Visual Acuity,

\begin{tabular}{|c|c|c|c|c|c|}
\hline \multirow[b]{2}{*}{$\begin{array}{l}\text { Follow-up } \\
\text { (Months) }\end{array}$} & \multicolumn{2}{|c|}{ Mean (SD)* } & \multirow[b]{2}{*}{$\begin{array}{c}\text { Cutoff } \\
\text { Point } x \dagger\end{array}$} & \multirow[b]{2}{*}{$\begin{array}{c}A_{t} \text { Above Cutoff } \\
\text { Point }(\%) \ddagger\end{array}$} & \multirow[b]{2}{*}{$\begin{array}{c}\text { TAE Above Cutof } \\
\text { Point (\%)§ }\end{array}$} \\
\hline & $\begin{array}{c}\text { Ranibizumab } \\
\text { Group }(\mathrm{n}=238)\end{array}$ & $\begin{array}{c}\text { Sham Group } \\
(\mathrm{n}=238)\end{array}$ & & & \\
\hline 1 & $3.9(10.2)$ & $-0.2(8.6)$ & 4.9 & 46 & 40 \\
\hline 3 & $5.9(10.5)$ & $-3.7(11.3)$ & 0.4 & 70 & 49 \\
\hline 6 & $6.5(11.8)$ & $-6.6(13.0)$ & -0.9 & 73 & 55 \\
\hline 12 & $7.2(14.6)$ & $-10.4(15.1)$ & -1.9 & 73 & 61 \\
\hline 24 & $6.6(17.2)$ & $-14.9(18.8)$ & -5.0 & 75 & 60 \\
\hline
\end{tabular}

ETDRS, early treatment diabetic retinopathy study; SD, standard deviation.

* Standard deviation calculated using standard errors (SE) from the trial report: SD $=\mathrm{SE} \cdot \sqrt[V]{ } \mathrm{n}$.

$†$ Point above which for every change in visual acuity a proportion of the treated patients achieve their change due to the treatment.

‡ Treated patients who ended above cutoff point.

$\$$ Treated patients who ended above cutoff point due to treatment. 
Table 2. Results per Effect Modifier in the MARINA Trial.

\begin{tabular}{|c|c|c|c|c|c|c|}
\hline \multirow[b]{2}{*}{ Effect Modifier } & \multirow[b]{2}{*}{ Subgroup } & \multirow{2}{*}{$\begin{array}{c}\text { No. in } \\
\text { Treated / } \\
\text { Reference } \\
\text { Group }\end{array}$} & \multicolumn{2}{|c|}{$\begin{array}{l}\text { Change in Visual Acuity } \\
\text { at } 24 \text { Months, Mean (SD)* }\end{array}$} & \multirow[b]{2}{*}{$\begin{array}{c}\text { Cutoff } \\
\text { point } x \dagger\end{array}$} & \multirow[b]{2}{*}{$\begin{array}{l}\text { TAE } \\
\text { (\%) }\end{array}$} \\
\hline & & & $\begin{array}{c}\text { Ranibizumab } \\
\text { Group }\end{array}$ & $\begin{array}{l}\text { Sham } \\
\text { Group }\end{array}$ & & \\
\hline \multirow[t]{4}{*}{ Age } & $50-64 y$ & $16 / 11$ & $6.1(21.2)$ & $-13.7(23.9)$ & -6.2 & 48 \\
\hline & $65-74 y$ & $64 / 67$ & $7.2(15.8)$ & $-11.9(19.7)$ & -4.8 & 54 \\
\hline & $75-84 y$ & $124 / 132$ & $7.6(16.4)$ & $-16.0(19.0)$ & -5.3 & 64 \\
\hline & $\geq 85 \mathrm{y}$ & $36 / 28$ & $1.9(16.4)$ & $-16.8(19.3)$ & -9.4 & 54 \\
\hline \multirow[t]{4}{*}{ Initial visual acuity } & $20 / 160$ or worse & $48 / 51$ & $10.6(17.5)$ & $-0.8(13.3)$ & 9.1 & 57 \\
\hline & $20 / 100$ to $20 / 125$ & $59 / 50$ & $9.3(15.4)$ & $-13.6(16.1)$ & -2.4 & 69 \\
\hline & $20 / 63$ to $20 / 80$ & $68 / 72$ & $5.4(16.2)$ & $-20.0(17.6)$ & -7.7 & 69 \\
\hline & $20 / 50$ or better & $65 / 65$ & $1.8(15.8)$ & $-21.3(19.8)$ & -11.4 & 61 \\
\hline \multirow[t]{4}{*}{ CNV lesion size, DA } & $\leq 2$ & $39 / 46$ & $10.2(14.2)$ & $-13.4(18.2)$ & -2.9 & 66 \\
\hline & $>2$ to $\leq 4$ & 86 / 77 & $9.7(14.4)$ & $-15.5(18.7)$ & -4.0 & 68 \\
\hline & $>4$ to $\leq 6$ & $63 / 60$ & $3.8(20.0)$ & $-15.0(18.3)$ & -4.3 & 57 \\
\hline & $>6$ & $52 / 55$ & $2.1(16.7)$ & $-15.5(20.7)$ & -9.8 & 49 \\
\hline \multirow[t]{2}{*}{ CNV lesion type } & Minimally classic & $91 / 87$ & $6.4(20.0)$ & $-14.7(17.3)$ & -2.6 & 64 \\
\hline & Occult & $149 / 150$ & $6.2(14.7)$ & $-15.3(19.5)$ & -6.6 & 59 \\
\hline
\end{tabular}

$C N V$, choroidal neovascularisation; $D A$, number of disc areas; ETDRS, early treatment diabetic retinopathy study; $S D$, standard deviation.

* Standard deviation calculated using 95\% confidence intervals (95\% CI) from the trial report: SD = (upper limit - lower limit) / $3.92 \cdot \sqrt{ }$. for $\mathrm{n} \geq 60$; $\mathrm{SD}=$ (upper limit - lower limit) / $4.128 \cdot \sqrt{ } \mathrm{n}$ for $\mathrm{n}<60$. $\dagger$ Point above which for every change in visual acuity a proportion of the treated patients achieve their change due to the treatment.

The means and SDs for calculating the cutoff points for the following examples are shown in Table 3. For IOP lowering with bimatoprost after 29 days, the cutoff point is located at an IOP reduction of 16\% (see Figure 2A). For treating refractory diabetic macular edema with intravitreal triamcinolone, after 2 months, the cutoff point is located at approximately 0.06 LogMAR units visual acuity improvement (Figure 2B). After treatment of convergence insufficiency with vergence/accommodation therapy, the cutoff point is located at approximately $7 \mathrm{~cm}$ reduction of the NPC (Figure $2 \mathrm{C}$ ). Figure 2 shows that the proportion of treated patients who achieved a change in the outcome better than the cutoff point attributable to the treatment is greatest in the bimatoprost trial report data, followed by the vergence/accommodation therapy trial and the triamcinolone report data. 
Table 3. Assumptions for Calculating Cut-off Points for Deciding Whether to Continue or Stop a Treatment.

\begin{tabular}{|c|c|c|c|c|c|}
\hline \multirow[b]{2}{*}{ Study } & \multirow[b]{2}{*}{ Outcome } & \multirow[b]{2}{*}{$\begin{array}{l}\text { No. in Treated / } \\
\text { Reference Group }\end{array}$} & \multirow[b]{2}{*}{ Follow-up } & \multicolumn{2}{|c|}{$\begin{array}{c}\text { Change in Outcome, } \\
\text { Mean (SD) }\end{array}$} \\
\hline & & & & $\begin{array}{l}\text { Treated } \\
\text { Group }\end{array}$ & $\begin{array}{c}\text { Reference } \\
\text { Group }\end{array}$ \\
\hline DuBiner et al. ${ }^{5}$ & IOP reduction (\%) & $21 / 21$ & $\begin{array}{l}\text { Day 29, at } \\
12 \text { noon }\end{array}$ & $30.1(12.4)^{*}$ & $2.1(16.5)^{*}$ \\
\hline Dehghan et al. ${ }^{6}$ & $\begin{array}{l}\text { Visual acuity } \\
\text { (LogMAR) }\end{array}$ & $43 / 45$ & 2 months & $0.13(0.27)^{*}$ & $0.02(0.26)^{*}$ \\
\hline CITT $^{7}$ & $\begin{array}{l}\text { NPC break } \\
\text { reduction }(\mathrm{cm})\end{array}$ & $60 / 54$ & 12 weeks & $10.4(5.07) \dagger$ & $3.9(4.98) \dagger$ \\
\hline
\end{tabular}

CITT, Convergence Insufficiency Treatment Trial; IOP, intraocular pressure; NPC, near point of convergence; SD, standard deviation.

* Standard deviation calculated using standard errors (SE) from the trial report: SD $=\mathrm{SE} \cdot V_{\mathrm{n}}$.

$\dagger$ Standard deviation calculated using 95\% confidence intervals (95\% CI) from the trial report: SD = (upper limit - lower limit) $/ 4.128 \cdot \sqrt{ } \mathrm{n}$.

\section{A. Patients with elevated intra-ocular pressure}

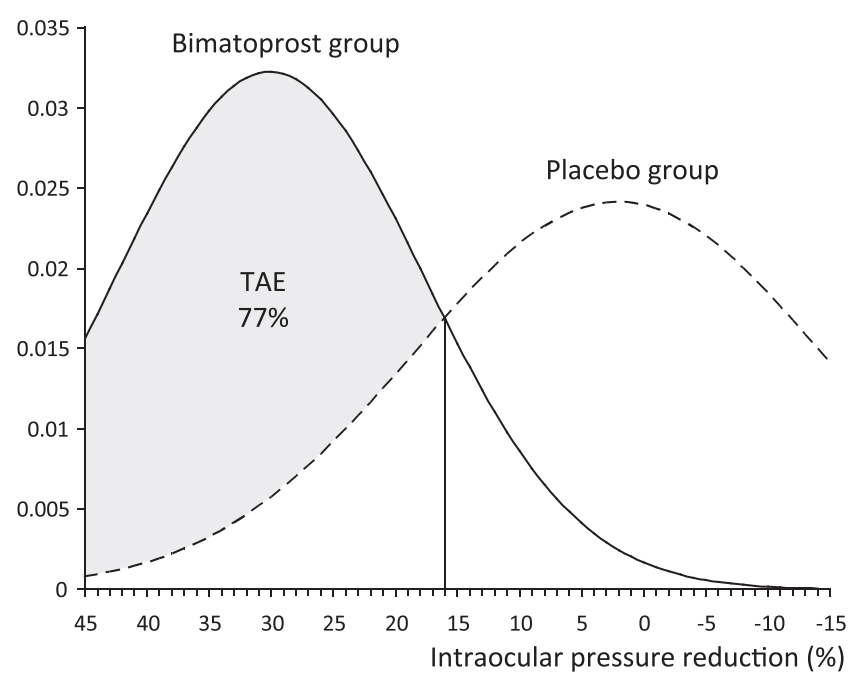

Figure 2. Normal distribution models of clinical trial results: Patients with high intraocular pressure (A), with refractory diabetic oedema (B), and convergence insufficiency $(\mathbf{C})$. The intersections of the treated group curve and the placebo curves are visible. The proportions of treated patients achieving a better change in the outcome than the cut-off point attributable to the treatment are depicted as shaded areas under the curves, with the size of the proportions indicated as a percentage (treatment attributed effect, TAE). 
B. Patients with refractory diabetic macular edema

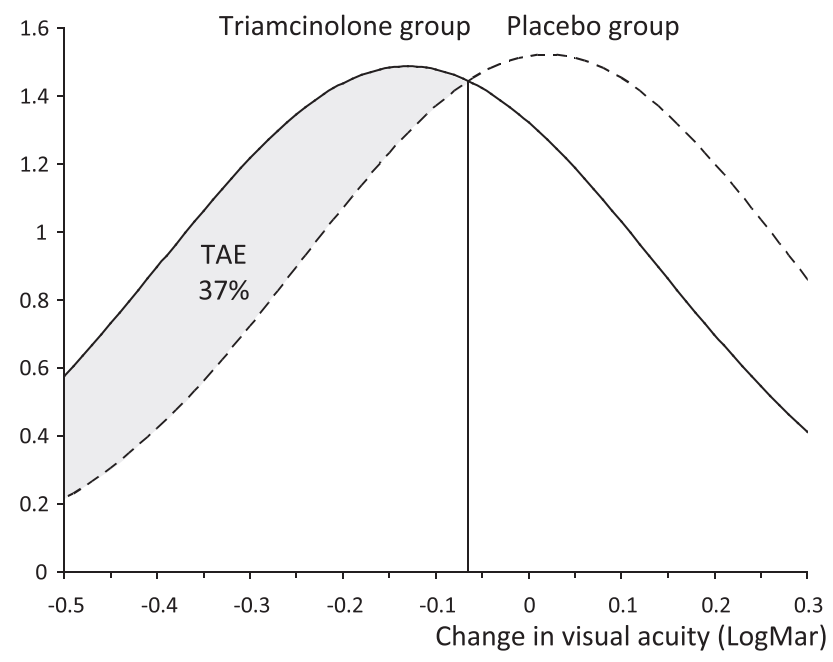

C. Patients with convergence insufficiency

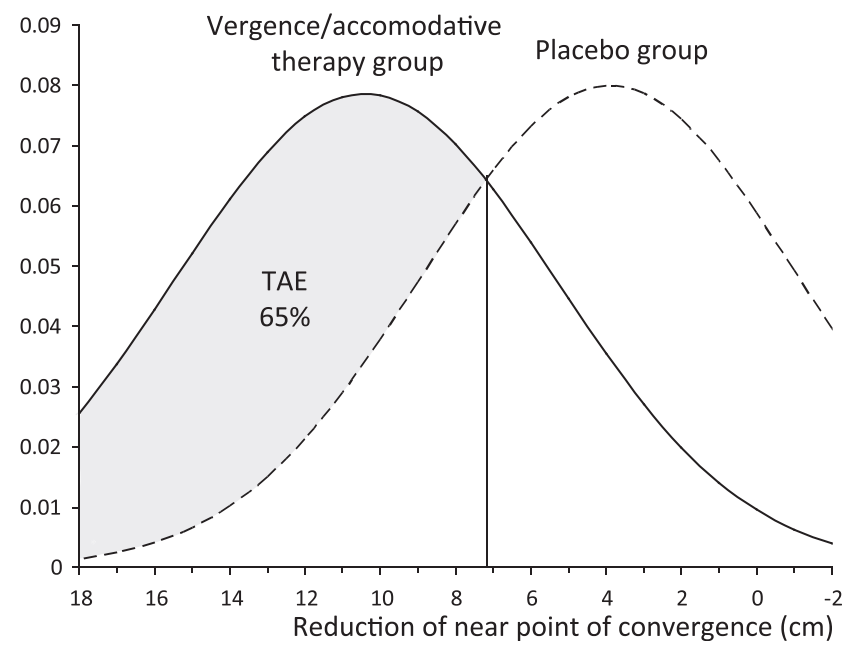

Figure 2. Continued. 


\section{Discussion}

In this article we have analysed the change in continuous outcome variables used in the follow-up of chronic disease during a treatment. We developed a new, straightforward method to calculate cutoff points above which, for every level of change, it is certain that some proportion of the treated patients achieved that change due to the treatment. The nAMD example illustrates several relevant points regarding this method. We discuss these points in the following sections.

\section{This method produces a cutoff point that limits the range of clinically sound cutoff points}

Clinicians may seek a cutoff point to decide to continue or stop a treatment for patients in their practice. It is important that using such a cutoff point does not lead to stopping the treatment in patients in whom the treatment is effective. As illustrated in the nAMD example, the cutoff point at the intersection of the curves is the point at which for every smaller decline or greater improvement in visual acuity, a proportion of the treated patients has achieved the change attributable to the treatment. Stopping the treatment is therefore not appropriate in patients with a change better than the cutoff point at the intersection. The choice of a cutoff point used in clinical practice is therefore limited as it should not be located above the cutoff point calculated by this method.

\section{The value of the cutoff point depends on the size and the distribution of the treatment effect in} the research population

The mean change in the outcome defines the position of the "treatment curve" such as depicted in Figure 1. The SD of the change in the outcome defines the width of the curve. The intersection of the treatment curve with the sham curve is dependent on the shape and position of the treatment curve (and the placebo curve). The cutoff point occurring at the intersection of the curves is therefore dependent on the efficacy of the treatment and its distribution width.

\section{A limited decline in visual acuity can reflect a beneficial effect of therapy}

As we show in the nAMD example, the cutoff point may not be equal to a zero change in outcome. At 24 months of follow-up, a decline of 5 letters is the cutoff point. This implies that some patients may show a loss (of up to 5 letters) in visual acuity but nevertheless be better off than would have been the case if they had not been treated. In other words, the treatment has limited the deterioration of visual acuity. Intuitively, and in daily practice, "no change" is often interpreted as "no effect." Our example shows that such an interpretation may not be in accordance with the evidence. 


\section{The cutoff point may change over time}

Table 1 shows that the cutoff point changes over time in the nAMD example. This is because visual acuity in the sham group, and, initially, in the treated group, is not stable but alters over time. It is important to know the natural history of the outcome and the time period for the effect of the treatment to be at a maximum. For example, in IOP therapy this occurs fairly soon, while for the visual acuity change in $\mathrm{AM}$ D, it takes longer. The example of nAMD shows that, even after a short time period, a cutoff value can be given such that above this value one can be certain that this change of visual acuity is achieved by the intervention. Changing or stopping treatment above the cutoff point may not be the best choice. Below this value, one can consider stopping or changing the therapy or wait for the development of the visual acuity and act according to the new cutoff value for that time period. Of course, other considerations for changing or stopping a treatment should be taken into account as well.

\section{The cutoff point may differ among different levels of effect modifiers}

The presented method can be of more practical value when subgroup analyses have been conducted so that the method can be applied per subgroup. In that case, a more patient-specific cutoff point can be selected for use in clinical practice. Table 2 shows that the cutoff point is different among groups with different age, initial visual acuity, CNV lesion size, and CNV lesion type in the nAMD example, based on subgroup analysis results. In older patients, in patients with a good initial visual acuity, and in patients with large lesions, a loss of visual acuity of up to around 10 letters can still be an effect of the treatment in a proportion of patients. In occult lesions, the visual acuity loss may be more severe than in minimally classic lesions and still be an effect of the treatment in a proportion of patients.

\section{Application to other scales and conditions}

The method described here can be applied to scales and conditions other than the change in visual acuity in $\mathrm{nAMD}$. In addition, the method may be even more appropriate in the case of a continuous variable that is altered by a treatment but that has a stable natural course over a (relatively short) period of time. Examples of conditions with such outcomes are elevated IOP and convergence insufficiency. For IOP lowering with bimatoprost after 29 days as presented by DuBiner et al., the cutoff point can be calculated to be an IOP reduction of $16 \%$. This means that, for a reduction in the IOP of more than $16 \%$ after 29 days, a proportion of treated patients will achieve that lowering because of treatment with bimatoprost. The European Glaucoma Society states that if the first choice monotherapy is not effective on IOP, it is preferable to switch to another agent. "Treatment is considered 'effective' on the IOP when the observed IOP lowering effect on the treated patient is comparable to the published average 
effect of the same compound on a similar population." ${ }^{\prime \prime}$ It is unclear what evidence this statement is based on. Based on meta-analysis, the published average effect of bimatoprost is $\geq 27 \%$ IOP reduction. ${ }^{9}$ If $27 \%$ IOP reduction is assumed as a cutoff point in clinical practice, instead of $16 \%$ as determined from the method example, this means that the treatment will be stopped in patients in whom bimatoprost treatment was effective in reducing IOP. However, the clinical appropriateness of the method is also influenced by the implications involved in withholding a treatment. For instance, the decision to discontinue a treatment for high IOP or hypertension can be made relatively easy since there are several other treatments to choose from. Application of the method to the effect of intravitreal triamcinolone on diabetic macular edema results in a cutoff point close to zero change. The cutoff point of 0.06 (an improvement in visual acuity) suggests that, at 2 months follow-up, a small improvement needs to be observed in order to decide that the treatment has been effective. In this example, "no change" seems to imply "no effect," with a value better than "no change" being the cutoff point. The convergence insufficiency example shows that, according to the results in the trial report used, an improvement of the NPC may occur in an ineffective treatment. Only a reduction of the NPC of more than $7 \mathrm{~cm}$ indicates that patients clearly achieved this reduction due to the treatment.

In order to apply the method as presented here, input data should be based on RCT data. In such studies, a group that does not receive the treatment, with participants randomly assigned to this arm, should be included in order to obtain an unconfounded estimate of the change in the outcome variable without treatment. It is possible to also employ the method to assess the usefulness of switching from one treatment to another. Some caution is warranted in this case. When there are small differences between the effects of the treatments, with relatively large SDs, then the TAE will be relatively small above the default cutoff point or above any other cutoff point. This implies that it is difficult to attribute a change in outcome measure, after a change in treatment, to the treatment change in this situation.

A way of proving that the choice of a cutoff point is appropriate would be to re-randomise those patients below the cutoff point (in whom the treatment is less likely to be effective) either to stop or to continue treatment and to compare the outcome in the two new groups. If further treatment would be ineffective in these patients, the outcome would not differ significantly between the groups, and stopping the treatment below the cutoff point would have been warranted at the moment of re-randomisation. There may be a group of apparent nonresponders who are actually responders with an aggressive natural course that the treatment cannot counteract. In the patients selected for the trial, one half will show the natural (aggressive) course and the other half the course in case treatment 
is continued, and the proposed trial would show whether continuing treatment in the apparent "nonresponding" patients would have been warranted. Regression to the mean may be an issue here, as it will cause the last measurement before re-randomisation to be closer to its real value than the initial measurement. This may add to the observation that the change in visual acuity after re-randomisation is less dramatic than the change before re-randomisation, with the treatment appearing less effective in the re-randomised population.

Using cutoff points calculated by this method may also lead to the design of safer stopping trials, in which the treatment is (randomly) stopped or continued only in those patients below the calculated cutoff point instead of randomising all patients to either stopping or continuing treatment. It can be expected that this will prevent the reoccurrence or exacerbation of the treated disease to a greater extent in those whose treatment was discontinued compared with randomising all patients. ${ }^{10}$ If we were to hypothetically rerandomise the MARINA trial patients at 24 months, $25 \%$ of the treated patients would be re-randomised, since the proportion of treated patients who ended above cutoff point was $75 \%$ (see Table 1).

An issue remains regarding the variability of the estimate of the cutoff point. One can expect that the value will differ between similar RCTs. This would be a phenomenon comparable to the variability of the effect estimates between similar RCTs, for example, the difference in the relative risks or the difference in continuous outcomes. Further research could focus on developing methods to calculate the statistical variance of the cutoff point and to combine the estimates based on several RCTs.

Furthermore, in employing a cutoff point in clinical practice, one must assess whether the measured change in the outcome is likely to be real. When a clinician suspects that the observed change in outcome is due to measurement error (frequently the case in single IOP measurements) then basing decision making on that particular set of measurements would not be warranted. Considering repeating the measurement should be an issue in every assessment of treatment effect.

For the examples, we used the mean and SD derived from the trial reports. Ideally, the original data from individual patients should have been used since it is likely that the distributions are skewed, but we did not have access to these data. These results should therefore only be used as an illustration of the method and should not be used as the actual cutoff points for stopping or continuing ranibizumab treatment for nAMD, and similarly for the other treatments and conditions mentioned. Regarding nAMD, one should also keep in mind that follow-up decisions are not solely based on the change in 
one outcome, such as visual acuity. For instance, decision-making may also be based on the finding of unresolved macular edema on optical coherence tomography. However, again, this may raise the question of how much a change in the outcome - macular edema - reflects the effect of treatment.

Our method can be readily applied to other trial data. In the monitoring of any chronic (ophthalmic) disease, appropriate evidence-based cutoff points are of great value to clinicians. Such results will contribute to evidence-based monitoring, which has not been studied extensively.1,12 It is possible that a similar method has been described before, but we are unaware of such a publication. We recommend using this method in future RCTs in which a change in an outcome versus baseline is being studied, for example, visual acuity, IOP, or flare (in uveitis). We recommend presenting the calculated cutoff point as well as the proportion of treated patients ending above this cutoff point. 


\section{References}

1. Rosenfeld PJ, Brown DM, Heier JS, et al. Ranibizumab for neovascular age-related macular degeneration. N Engl J Med. 2006;355:1419-1431.

2. Klaver CC, Wolfs RC, Vingerling JR, Hofman A, de Jong PT. Age-specific prevalence and causes of blindness and visual impairment in an older population: the Rotterdam Study. Arch Ophthalmol. 1998;116:653-658.

3. The Cochrane Collaboration. Data extraction for continuous outcomes, Chapter 7.7.3. In Cochrane Handbook for Systematic Reviews of Interventions. Available at: http://www. cochrane-handbook. org/. Accessed March 2011.

4. Boyer DS, Antoszyk AN, Awh CC, Bhisitkul RB, Shapiro H, Acharya NR. Subgroup analysis of the MARINA study of ranibizumab in neovascular age-related macular degeneration. Ophthalmology. 2007;114:246-252.

5. DuBiner H, Cooke D, Dirks M, Stewart WC, VanDenburgh AM, Felix C. Efficacy and safety of bimatoprost in patients with elevated intraocular pressure: a 30-day comparison with latanoprost. Surv Ophthalmol. 2001;45(suppl 4);S353-360.

6. Dehghan MH, Ahmadieh H, Ramezani A, Entezari M, Anisian A. A randomized, placebo-controlled clinical trial of intravitreal triamcinolone for refractory diabetic macular edema. Int Ophthalmol. 2008;28:7-17.

7. Convergence Insufficiency Treatment Trial Study Group. Randomized clinical trial of treatments for symptomatic convergence insufficiency in children. Arch Ophthalmol. 2008;126:1336-1349.

8. Norquist G, McGuire TG, Essock SM. Cost-effectiveness of depression treatment for adolescents. Am J Psychiatry. 2008; 165:549-552.

9. Van der Valk R, Webers CA, Schouten JS, Zeegers MP, Hendrikse F, Prins MH. Intraocular pressurelowering effects of all commonly used glaucoma drugs: a meta-analysis of randomized clinical trials. Ophthalmology. 2005;112:1177- 1185.

10. Walma EP, Hoes AW, van Dooren C, Prins A, van der Does E. Withdrawal of long-term diuretic medication in elderly patients: a double blind randomised trial. BMJ. 1997;315: 464-468.

11. Glasziou P, Irwig L, Aronson J. Evidence-Based Medical Monitoring: From Principles to Practice. Oxford: Blackwell Publishing Ltd.; 2008.

12. Glasziou P, Irwig L, Mant D. Monitoring in chronic disease: a rational approach. BMJ. 2005;330:644648. 
4.
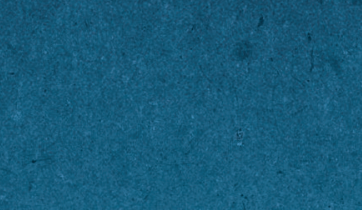

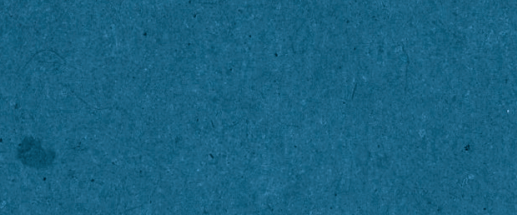
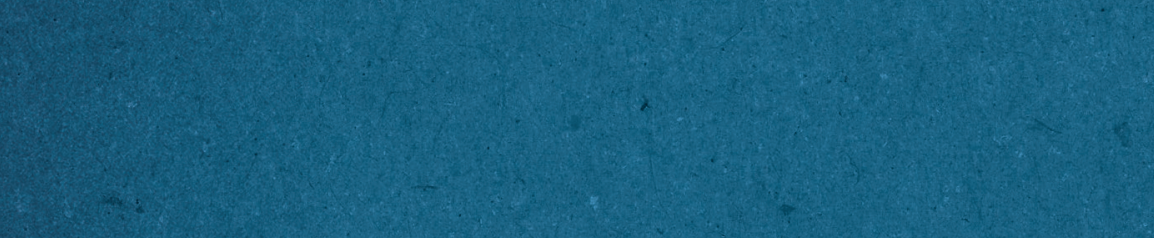

8

$y 3$

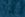




\section{Chapter 6}

Distinguishing between better and worse visual acuity by studying the correlation with quality of life in neovascular age-related macular degeneration

Mari Elshout, Margriet I. van der Reis, Yvonne de Jong-Hesse,

Carroll A.B. Webers, Jan S.A.G. Schouten

Ophthalmology 2016; 123: 2408-2412 


\section{Abstract}

Purpose: To determine whether there is a level of visual acuity (VA) in neovascular age-related macular degeneration (nAMD) above which the correlation of VA with disease-related quality of life (QoL) is significantly greater than below this level.

Design: An observational, cross-sectional study. Participants: A total of 184 patients with nAMD aged at least 50 years were included in the study.

Methods: In face-to-face interviews, we assessed QoL with the Macular DiseaseDependent Quality of Life (MacDQoL) questionnaire. We measured VA with standardised Radner reading charts. We used regression splines analysis with a single hinge point, with the MacDQoL score as the dependent variable and VA as the independent variable. The $\mathrm{x}$-coordinate (VA) of the hinge point was varied and tested with each iteration. A second method of regression splines analysis was also performed, without a preset hinge point.

Main Outcome Measures: The primary outcome measure is the cutoff point at or below which VA is associated with significantly less change in QoL than above this cutoff. The linear coefficients below and above the cutoff are defined as change in MacDQoL score per logarithm of the minimum angle of resolution (logMAR) unit of change in VA.

Results: With Snellen equivalent VA 0.05 (1.3 logMAR) or worse, the linear coefficient was 0.15 . With VA better than 0.05 , the linear coefficient was 2.40 (P value of the difference: 0.009).

Conclusions: When VA is above 0.05 , there is a stronger and significant relation between VA and QoL. At or below this level, the relation between VA and QOL approaches zero. With better VA, a difference in VA implies a significant difference in QoL. With poorer VA, a difference in VA is unlikely to imply a difference in QoL. Therefore, in treating nAMD, the aim should be to keep Snellen VA above 0.05 to have an impact on QoL. If it is certain that the best-corrected VA below 0.05 is permanent, these findings imply there may be less, if any, benefit to continue further treatment. This is to be evaluated on a case-by-case basis. 


\section{Introduction}

Neovascular age-related macular degeneration (nAMD) causes significant vision loss and has a major impact on patients' quality of life (QoL)., 2 Treatment with intravitreal administration of anti-vascular endothelial growth factor (anti-VEGFs) improves visual acuity (VA) and improves QoL. ${ }^{3}$ Criteria to start treatment and the criteria to re-treat are well established. Criteria to stop treatment have been described in guidelines. ${ }^{4-6}$ The criteria to stop treatment are based on the notion that permanent structural damage to the fovea can render the treatment ineffective in improving VA. Visual acuity is a factor in the decision to stop or continue a treatment. The quality and empirical support of criteria to stop treatment are important. Inadequate criteria may lead to under- and/ or overtreatment. On one hand, they can lead to withholding a potentially effective treatment. On the other hand, they may expose patients to the risk of unnecessary treatment. Further, overtreatment does not contribute to cost-effective practice, especially in the case of costly drugs such as certain anti-VEGFs. The importance of the criteria to stop treatment contrasts with the lack of empirical evidence that should underpin the criteria used in guidelines.

In clinical practice and in clinical research, VA is used to monitor treatment effect. Improvement of VA however, is not the ultimate goal when treating patients. Improving VA improves disease-related quality of life QoL (QoL), which should be the ultimate goal of a treatment. An improvement in VA must lead to an improvement in QoL in order to be relevant. Therefore, the correlation between VA and QoL is important. The shape of the relationship between visual function and QoL has previously been investigated. In one study, health-related QoL was measured using the National Eye Institute Visual Functioning Questionnaire. The relationship VA-QoL tended to plateau at low levels of visual function.? If no correlation exists between VA and QoL below a certain level of VA (a cutoff), it is important to keep VA above this cutoff with treatment to have an impact on QoL. As an example of such a cutoff point, an earlier study recommended to keep VA at 20/200 rather than 20/400, based on basic functional performance tests among patients with low VA. ${ }^{8}$

In this study, we assess the correlation of VA with scores on the disease-specific QoL measure MacDQoL, in order to identify a cut-off value in VA, below which less difference in QoL can be expected with a difference in VA. This could imply, that when VA remains permanently below this point after maximum treatment effect, treatment benefits conferred in QoL are less apparent than above this point, even when there is an impact on VA. Therefore, in clinical practice, the aim should be to keep the VA above this level to have an impact on QoL. 


\section{Methods}

\section{Design, participants and setting}

We performed a cross-sectional, observational study. The study enrolled patients from the University Eye Clinic Maastricht; the Catharina Hospital Eindhoven; ZorgSaam Hospital Zeeuws-Vlaanderen, and the VU University Medical Center in Amsterdam, in The Netherlands. The Medical Ethical Committees in these centers approved the study according to Dutch law and the Declaration of Helsinki. Patients aged at least 50 years and diagnosed with nAMD with choroidal neovascularisation (CNV) on fluorescein angiography between 1992 and 2011 were eligible. We selected patients between September 2009 and November 2011. We screened 1948 files; 588 patients were eligible. Of these, 184 patients agreed to participate in the study and gave written informed consent before taking part. Interviews were taken between March 2010 and November 2011.

\section{Data Collection}

Trained researchers interviewed patients at the patients' homes. We measured VA before the interview using validated Radner Reading cards (Dutch version) for each eye separately and both eyes simultaneously., ${ }^{90}$ We used the MacDQoL questionnaire to assess diseasespecific quality of life." We administered the visual tests and questionnaires using a standard protocol. We collected clinical data from the medical records to describe the study population.

\section{Visual Acuity Tests}

We measured VA with Radner reading charts. We used different charts for the right eye, the left eye, and for binocular VA. We measured the distance used to read the Radner chart and used it to calculate the logarithm of the reading acuity determination (logRAD). Counting fingers and seeing hand motions were assigned the appropriate values on the decimal visual acuity scale. We converted both LogRAD and decimal VA to LogMAR visual acuity equivalents for linear regression analysis.

\section{Macular Disease-Dependent Quality of Life Score}

The MacDQoL is a measure of the impact of macular disease on QoL. It investigates individuals' views of the impact of macular disease on life domains commonly affected by macular disease, along with the importance of each domain to the individuals' QoL. The MacDQoL has good face and content validity and evidence of good internal consistency, reliability and construct validity. ${ }^{12}$ 


\section{Statistical Analysis}

We used descriptive analyses to characterise the sociodemographic and clinical characteristics, and the QoL scores of the patients. We used linear regression to quantify the relation between VA in the better-seeing eye and QoL. We used 2 approaches to assess for a cutoff point in VA where a change in the relation between VA and QoL occurs. These methods are described in the following.

\section{Regression splines with given hinge points}

We used SPSS release 20.0 (2011, IBM Corporation, Armonk, NY) to run regression splines analysis. This type of analysis has been described previously. ${ }^{13-15}$ It can be used to assess whether a change in slope occurs in a linear regression model at a given "hinge point," that is, a transition point at which the slope is thought to change. We used this method to apply regression splines models to a range of hinge points for VA in the better-seeing eye versus QoL. Several factors can indicate that the hinge point marks a cutoff point in VA below which QoL changes less per unit of VA than it does above it. These factors are a large difference in the slopes below and above the hinge point, a high $\mathrm{R}^{2}$ of the total model, or a low $P$ value of the difference in the slopes.

\section{Regression splines with unknown hinge points}

We also used another approach for the analysis, without pre-setting the location of the hinge point. The analysis provides the best fit of two slopes with their hinge point, if present. For this analysis we used the $R$ language and environment ( $\mathrm{R}$ version 3.2.1, The Foundation for Statistical Computing, 2015), with the Segmented package for R to fit piecewise regression models. ${ }^{16}$ The package is designed to evaluate for a hinge point in the regression, i.e. a point at which the slope of the regression changes. Pre-setting a hypothesised location of a hinge point is not required. We assessed which segment showed less change in QoL score per change in VA (better-seeing eye), and which segment showed more change in the score per VA change. We described the hinge (cutoff point) at which this transition occurred. 


\section{Results}

A total of 184 patients completed the visual tests and interviews. There were no differences in age, gender or geographic location between those who participated and those who did not participate. Item completion rates exceeded 95\% for every measure. Comparison between patients with complete data and those who were excluded because of missing data showed no relevant differences for the rest of the variables. Table 1 shows the sociodemographic and clinical characteristics of the participants, as well as the QoL scores. Simple regression analysis showed that better VA was associated with higher QoL. Corrected for age and gender, MacDQoL score was 1.65 points better per LogMAR unit $(P=0.000)$.

Table 2 shows coefficients for QoL as a dependent of VA in the regression splines analysis with a range of pre-set hinge points. The coefficients are displayed for VA equal to or worse, and for VA better, than the corresponding hinge point. Adjustment for age and gender was applied. The $p$-value of the difference between the coefficients is displayed, as is the $\mathrm{R}^{2}$ of the total model. With a hinge point at 0.05 Snellen VA (1.3 LogMAR), the difference between the slopes has the lowest $P$-value. In addition, the $\mathrm{R}^{2}$ of the model produced is higher than with the other models. At VA equal to or worse than the hinge point, QoL difference per LogMAR unit is 0.15 points; at VA better than this hinge point is a factor 16 higher. We selected 0.05 Snellen VA as a cutoff point: at levels of VA equal or lower, there are much lower values for the strength of relation between VA and QoL than at better levels of VA.

Figure 1 shows the regression splines analysis without a present hinge point. It depicts VA in the better-seeing eye (LogMAR and Snellen equivalents) on the x-axis, and MacDQoL scores on the y-axis. At 0.04 Snellen VA, a transition occurs from a slope that is near zero, to a slope that indicating VA correlates with QoL. 
Table 1. Sociodemographic, clinical and quality of life characteristics of participants with neovascular age-related macular degeneration.

\begin{tabular}{|c|c|c|}
\hline & \multicolumn{2}{|c|}{$\mathrm{N}=184$} \\
\hline & Mean (SD) & \multirow{2}{*}{$\frac{\text { Range }}{52.3-99.4}$} \\
\hline Age, years & $81.2 \quad(7.1)$ & \\
\hline Female, \% & 52.2 & \\
\hline Disease duration, years & $5.8 \quad(3.4)$ & $1.0-16.0$ \\
\hline \multicolumn{3}{|l|}{ Better-seeing eye VA } \\
\hline LogMAR & $0.72 \quad(0.64)$ & $0.00-2.86$ \\
\hline Snellen & 0.19 & \\
\hline \multicolumn{3}{|l|}{ Fellow eye VA } \\
\hline LogMAR & $1.63 \quad(0.73)$ & $0.01-3.00$ \\
\hline Snellen & 0.023 & \\
\hline \multicolumn{3}{|l|}{ Binocular VA } \\
\hline LogMAR & $0.78 \quad(0.64)$ & $0.00-2.86$ \\
\hline Snellen & 0.17 & \\
\hline MacDQoL score & $-2.71 \quad(2.10)$ & -9.00 to 0.00 \\
\hline
\end{tabular}

LogMAR, Logarithm of the Minimum Angle of Resolution; SD, standard deviation; VA, visual acuity.

Table 2. Visual acuity versus disease-related quality of life. Regression splines analyses with pre-set hinge. Multiple iterations with varying hinges.

\begin{tabular}{lccccccc}
\hline & \multicolumn{2}{c}{$\begin{array}{c}\text { Hinge Point } \\
\text { Used (VA) }\end{array}$} & & \multicolumn{3}{c}{$\begin{array}{c}\text { MacDQoL Score Improvement per } \\
\text { LogMAR Unit Improvement: Slope }\end{array}$} \\
\cline { 2 - 3 } \cline { 5 - 7 } Iteration No. & Snellen & LogMAR & & Below Hinge & Above Hinge & $P$ value of Difference & $\mathrm{R}^{2}$ \\
\hline 1 & 0.025 & 1.6 & & -0.20 & 2.10 & 0.029 & 0.270 \\
2 & 0.032 & 1.5 & & -0.10 & 2.15 & 0.019 & 0.273 \\
3 & 0.040 & 1.4 & & 0.00 & 2.25 & 0.013 & 0.276 \\
4 & $0.050^{*}$ & 1.3 & 0.15 & 2.40 & 0.009 & 0.278 \\
5 & 0.063 & 1.2 & & 0.30 & 2.45 & 0.011 & 0.277 \\
6 & 0.079 & 1.1 & & 0.50 & 2.55 & 0.013 & 0.276 \\
7 & 0.100 & 1.0 & 0.65 & 2.60 & 0.020 & 0.273 \\
8 & 0.126 & 0.9 & 0.85 & 2.70 & 0.033 & 0.269 \\
9 & 0.158 & 0.8 & 0.95 & 2.80 & 0.040 & 0.268 \\
10 & 0.200 & 0.7 & 0.10 & 2.80 & 0.072 & 0.264 \\
11 & 0.251 & 0.6 & 1.25 & 2.90 & 0.109 & 0.261 \\
\hline
\end{tabular}

* Hinge point selected as cut-off point.

LogMAR, Logarithm of the Minimum Angle of Resolution. 


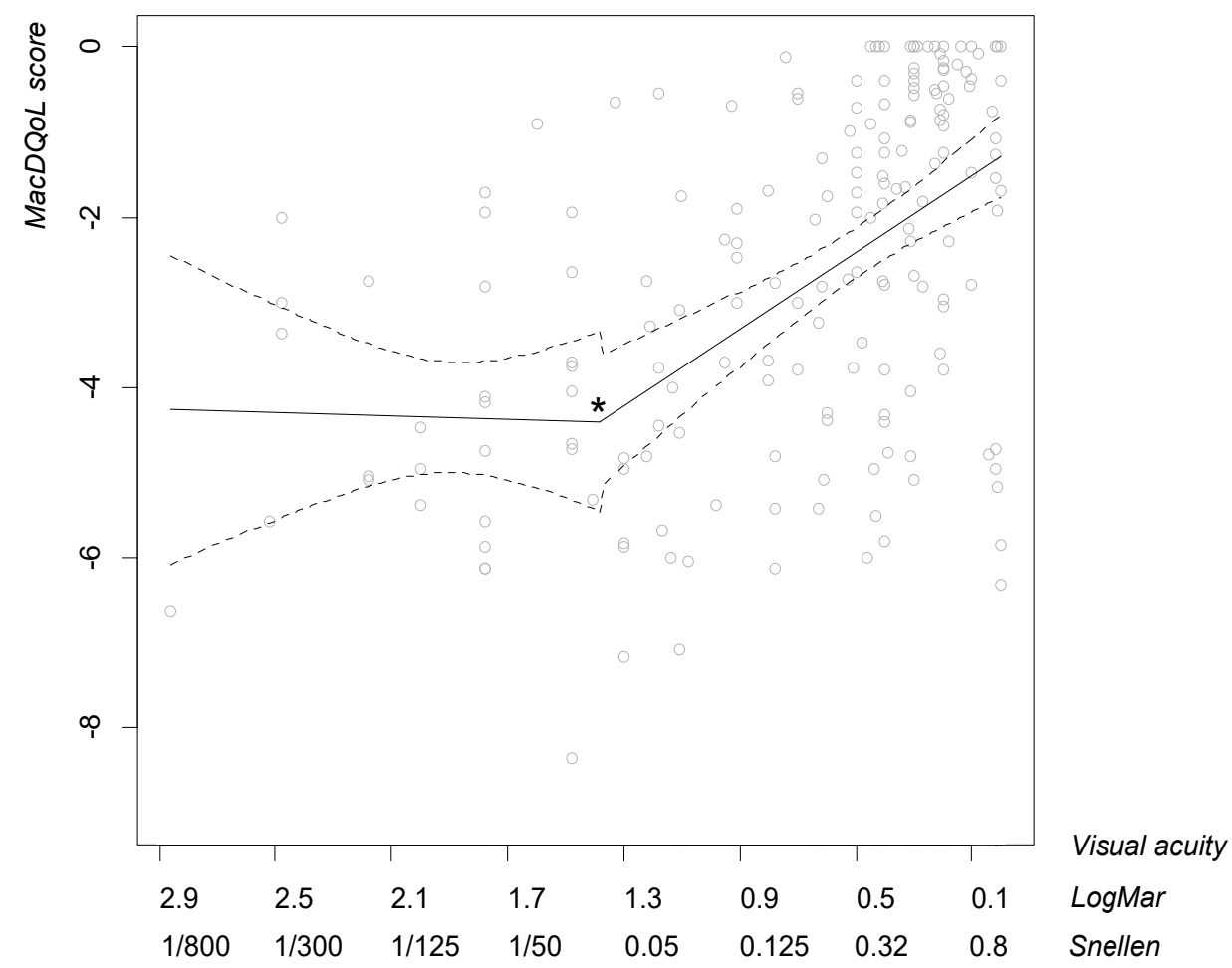

Figure 1. Regression splines analysis, visual acuity versus MacDQoL scores in neovascular age-related macular degeneration. Asterisk: cut-off point below which visual acuity is no longer correlated with quality of life. Dashed lines: 95\% confidence interval. 


\section{Discussion}

Neovascular age-related macular degeneration is associated with a lower QoL. With the MacDQoL questionnaire, this study confirmed that QoL score is higher with better VA in the better-seeing eye.

Linear splines regression showed there is a cutoff point in the relation of VA with QoL. The cut-off marks a change in slope of the relation between VA and QoL. The correlation of VA with QoL is less steep and close to zero with VA equal to or worse than the cutoff. The correlation is significantly larger with VA better than the cut-off. The cutoff occurred at 0.04 or 0.05 Snellen VA, depending on the statistical approach used. With VA being equal to or worse than 0.05 , different values of VA does not predict a different QoL. When VA deteriorates, it will not impact QoL. When VA improves but still remains worse than 0.05, there will be no impact on QoL.

The above suggests that when the VA remains permanently below this point, after a maximum treatment effect, treatment benefits conferred in terms of QoL are less apparent than above this point, even when there is an impact on VA. Improving VA or keeping VA stable above this point is likely to result in a quantifiable benefit in terms of QoL over losing VA and the aim of treatment should therefore be to keep VA above this level.

The cutoff point occurring in a lower rather than a higher region of VA has face validity. When VA falls from e.g. o.6 to 0.4 Snellen in the better-seeing eye, QoL understandably undergoes a significant reduction. A person may deteriorate from being able and allowed to drive to being unable and not allowed to do so. A person may deteriorate from being able to read a certain print size to not being able to do so. From being able to recognise faces at a certain distance, or not. In patients with a much lower VA, nAMD has caused more extensive damage. Visual acuity has fallen below a level were many capabilities such as driving, reading, and recognising faces are no longer possible. A further deterioration of VA will not affect these and other attributes anymore. These results align with prior research. Another study assessed the shape of the relationship of VA with health-related QoL, in photodynamic therapy-treated patients. It was found that the relationship had a relatively shallow gradient at the lower extreme of the visual function continuum?

The concept described bears clinical significance. It implies that the goal of treatment of nAMD should be to save as much VA as possible and at the least keeping VA better than 0.05 . This requires early recognition of nAMD and its recurrence. Early referral to an ophthalmologist, minimising treatment delay, and regular screening to detect recurrence of choroidal neovascularisation are needed. Either eye can become the better-seeing eye 
at some point. The fellow eye should be examined regularly for the presence of nAMD. In practical terms, when making clinical decisions, VA should be interpreted as bestcorrected VA in eyes without visually significant cataract or other treatable condition that influences VA.

Some guidelines recommend not starting therapy for eyes with a VA below a certain level. In addition, guidelines provide criteria to stop treatment. For instance, the Board of Ophthalmologists in the UK advises to discontinue treatment permanently if there is a reduction of BCVA in the treated eye to less than 15 letters (0.04 Snellen). ${ }^{4}$ The National Institute for Health and Care Excellence (NICE) do not recommend the antiVEGF ranibizumab when the best-corrected VA is below 6/96 ( $\approx 0.06$ Snellen VA). ${ }^{5}$ Dutch guidelines also use VA of 0.05 or worse as a criterion to stop treatment. An empirical underpinning for this criterion was not given. ${ }^{6}$

\section{Study limitations}

One could argue that a randomised controlled trial is needed to give definite evidence to further substantiate the recommendations. Such a trial would involve randomising participants with VA below a certain level to treatment and to no treatment. For ethical reasons, such a study is difficult to design. Based on our study one could think of including patients with VA definitely below 0.05. If ethical considerations can be overcome, a stopping trial could be considered. In the meantime, models such as presented here, based on observational studies, provide some support of the recommendations already formulated. In daily practice, it can be difficult to ascertain when the level of 0.05 Snellen VA is reached. The VA may still vary over time or there may be a temporary dip in VA due to other causes.

We studied the relation of VA and QoL and found less (or even no) relationship when VA is 0.05 or worse. If it is known that this level of VA is permanent, our findings imply there is little if any benefit to further treatment. Knowing if the level of VA is permanent was not studied. Clinicians will need to consider this on a case-by-case basis. In addition, in clinical practice, a starting point for the individual patient could be that the patient may experience and express the value of an injection with an anti-VEGF even when the VA changes only within the range below 0.05 .

In conclusion, we studied the relation of VA and QoL and find less (or even no) relationship when VA is 0.05 or worse. If it is known that this level of VA is permanent, our findings imply there is little, if any, benefit to be expected of further treatment. We did not study how to distinguish whether the VA level is permanent. Clinicians would certainly need to consider this on a case-by-case basis. 


\section{References}

1. Wong TY, Chakravarthy U, Klein R, et al. The natural history and prognosis of neovascular age-related macular degeneration: a systematic review of the literature and meta-analysis. Ophthalmology 2008;115(1):116-26.

2. Brown MM, Brown GC, Sharma S, et al. The burden of age-related macular degeneration: a valuebased analysis. Curr Opin Ophthalmol 2006;17(3):257-66.

3. Elshout M, van der Reis MI, Webers CA, Schouten JS. The cost-utility of aflibercept for the treatment of age-related macular degeneration compared to bevacizumab and ranibizumab and the influence of model parameters. Graefes Arch Clin Exp Ophthalmol 2014.

4. Ophthalmologists Scientific Department, Royal College of Ophthalmologists. Age-related Macular Degeneration - Guidelines for Management. London: Scientific Department - Royal College of Ophthalmologists, 2013.

5. Ranibizumab and pegaptanib for the treatment of age-related macular degeneration. NICE technology appraisal guidance 155 National Institute for Health and Care Excellence; 2008. Available at: https:// www.nice.org.uk/guidance/tal55. Accessed December 10, 2015.

6. Richtlijn Leeftijdgebonden Maculadegeneratie. Nederlands Oogheelkundig Gezelschap. Available at: www.oogheelkunde.org/richtlijnen. Accessed December 10, 2015.

7. Reeves BC, Langham J, Walker J, et al. Verteporfin photodynamic therapy cohort study: report 2: clinical measures of vision and health-related quality of life. Ophthalmology. 2009 Dec;116(12):2463-70.

8. Ebert EM, Fine AM, Markowitz J, et al. Functional vision in patients with neovascular maculopathy and poor visual acuity. Arch Ophthalmol 1986;104(7):1009-12.

9. Maaijwee KJ, Meulendijks CF, Radner W, et al. [The Dutch version of the Radner Reading Chart for assessing vision function]. Ned Tijdschr Geneeskd 2007;151(45):2494-7.

10. Radner W, Willinger U, Obermayer W, et al. [A new reading chart for simultaneous determination of reading vision and reading speed]. Klin Monbl Augenheilkd 1998;213(3):174-81.

11. Mitchell J, Bradley C. Design of an individualised measure of the impact of macular disease on quality of life (the MacDQoL). Qual Life Res 2004;13(6):1163-75.

12. Mitchell J, Wolffsohn JS, Woodcock A, et al. +Psychometric evaluation of the MacDQoL individualised measure of the impact of macular degeneration on quality of life. Health Qual Life Outcomes 2005;3:25.

13. Institute for Digital Research and Education. How can I run a piecewise regression in SPSS? Available at:http://www.ats.ucla.edu/stat/spss/faq/piecewise.htm. Accessed December 10, 2015.

14. IBM Corporation. Spline Regression with Estimated Knots in SPSS. Available at: http://www-ol.ibm. com/support/docview.wss?uid=swg21476694. Accessed December 10, 2015.

15. Smith PL. Splines As a Useful and Convenient Statistical Tool. The American Statistician 1979;33(2):5762.

16. Muggeo VMR. segmented: An R Package to Fit Regression Models with Broken-Line Relationships. R News 2008;8(1):20-5. 
4.
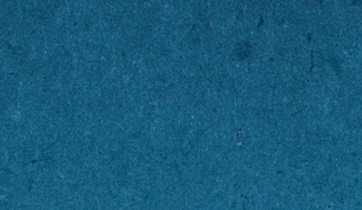

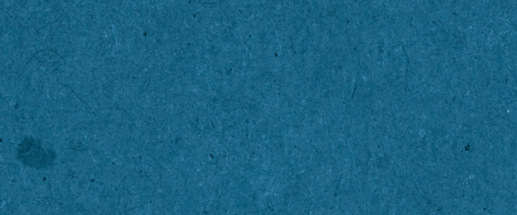
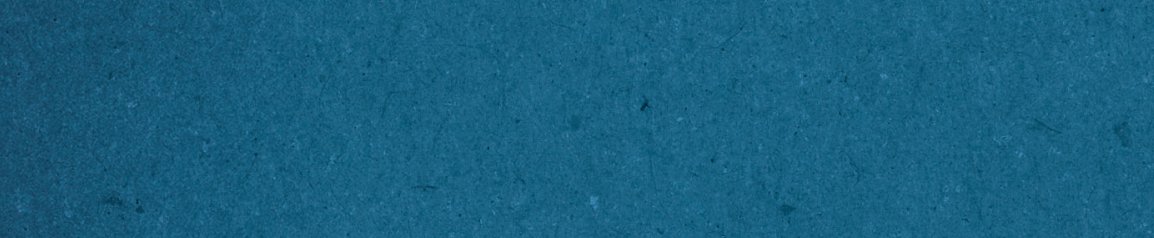

8

$y 3$

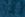




\section{Chapter 7}

\section{A systematic approach to evaluate practice based process- and outcome data applied to the treatment of neovascular age-related macular degeneration}

Margriet I. van der Reis*, Mari Elshout*, Tos T.J.M. Berendschot, Yvonne de Jong-Hesse, Carroll A.B. Webers, Jan S.A.G. Schouten.

*Both authors contributed equally to the manuscript

Submitted for publication 


\section{Abstract}

Background: In keeping with the principles of value-based health care, daily-practice eye care needs to be systematically evaluated in terms of its outcomes and processes. Using patient data regarding the treatment of neovascular age-related macular degeneration (nAMD), we illustrate an approach that can be used to support datadriven quality improvements.

Methods: In this cohort study, we reviewed medical records of consecutive patients with nAMD confirmed with fluorescein angiography (FA), and grouped patients by received treatment. All visual acuity (VA) measurements, ophthalmic exam results and treatment were recorded. Visual acuity was compared between groups using linear mixed model. Fluorescein angiograms were re-evaluated for appropriateness of the diagnosis. Outcome analysis was performed by (1) selecting the relevant outcome parameter: VA in logarithm of the minimal angle of resolution (LogMAR); (2) selecting the correct comparator and comparing the outcome parameter with this comparator: a historical untreated cohort and outcomes in the literature; (3) correcting for confounding variables (lesion type); and (4) identifying relevant process variables that determine the outcome. Process variables considered were severity of the disease at presentation, and doctor- and patient dependent process variables.

Results: In total, 473 eyes were included. At 12 months, change in VA was $0.54,0.48,0.09$, and 0.07 LogMAR in the no-treatment, photodynamic therapy (PDT), bevacizumab, and ranibizumab groups, respectively. Lesion type on FA differed between patient groups. Patient delay, wrong diagnosis and treatment intervals may have impacted outcomes.

Conclusions: PDT was likely not effective in daily practice. Anti-VEGFs were effective and appearing as effective as in randomised controlled trials, depending on the type of outcome analysis. Correct selection of a comparator cohort and addressing confounding, including confounding by indication and effect modification, are needed to achieve valid results and interpretation. Patient delay, diagnosis, indication for and application of treatment can potentially be improved to improve treatment outcomes. A systematic evaluation of the diagnosis, the indications for treatment and the processes and outcomes of new interventions in daily practice is needed at an early stage to improve the efficiency of their application. Such a systematic approach is needed to address issues raised in the era of value-based health care: to indicate opportunities to improve the value of care. 


\section{Background}

Ophthalmology, just as health care in general, is changing towards more transparency, accountability, and value-based principles of care and financing. In this regard, evaluation of the quality of care based on process and outcome data is vitally important. National registration programs and (electronic) medical records can supply a wealth of data to enable such evaluation. To improve their practice, ophthalmologists need to use such systems to analyse and interpret their process and outcome data.

However, ophthalmologists may not be familiar with this type of data analysis, the clinical epidemiological or statistical background, and/or interpretation of the results. We propose a systematic approach to analysing medical record data and interpret the results, to assess whether the outcomes can be improved and which process variables have affected the outcome.

As an example, we used data on neovascular age-related macular degeneration (nAMD) and interventions that have been introduced in the past years; photodynamic therapy (PDT) with verteporfin (VISUdyne ${ }^{\oplus}$, Novartis) and the anti-vascular endothelial growth factors (anti-VEGF) ranibizumab (Lucentis ${ }^{\oplus}$, Novartis) and bevacizumab (Avastin ${ }^{\circledR}$, Roche). 


\section{Methods}

Data on the treatment of nAMD was obtained in a multicenter, retrospective, comparative study, performed at two tertiary centers and two secondary eye centers across the Netherlands between 16 July, 2009 and 31 July, 2011. The Maastricht University Medical Center institutional review board and ethics committee approved the study and agreed that permission for this project was not necessary in accordance with the Dutch law of medical-scientific research. The coordinator at each clinical site approved the protocol for this study and gave consent to access clinical records.

We searched in our coded system of fluorescein angiographs (FA) for patients diagnosed between January 1, 1992, and December 31, 1997 to select nAMD patients for the "no treatment" natural history cohort. This period was selected, as during this period, patients with choroidal neovascularisation (CNV) usually received no treatment (sometimes laser photocoagulation for extrafoveal CNV). Furthermore, we selected and grouped consecutive patients who had been treated with PDT, bevacizumab or ranibizumab.

We applied the following inclusion criteria: evidence of CNV secondary to nAMD under the geometric center of the foveal avascular zone, identified on FA. For the treatment groups, patients had received at least one treatment with PDT, bevacizumab or ranibizumab. Patients were excluded if they had participated in an ophthalmic clinical trial. We selected one study eye based on the earliest diagnosis if both eyes were eligible.

For each subject, FAs were re-evaluated independently by two of four retina specialists (E.C. La Heij, F. Lion, F. Hendrikse or J.S. Schouten). They assessed the eligibility for inclusion; the appropriateness of the diagnosis and the indication for treatment; the pattern of the $\mathrm{CNV}$, the presence of non-CNV components, the size of the CNV, the size of the lesion (CNV plus non-CNV components), with the optic disc size as a reference. Disagreement was resolved by consensus. We adopted definitions from the Age-Related Eye Disease Study Research Group (AREDS) classification and the Macular Photocoagulation Study (MPS). ${ }^{1,2}$

Patients assigned to the PDT group had been treated according to the TAP protocol ${ }^{3}$ with a follow-up period of three months and a maximum of four PDT treatments per year. Patients assigned to the ranibizumab or bevacizumab groups were initially treated with at least one to three monthly injections. Retreatment criteria varied, partly reflecting early-day treatment strategies, and were based on the presence of leakage on FA, fluid on optical coherence tomography (OCT), macular hemorrhage, or vision loss due to nAMD, or a combination. Follow-up intervals ranged from one to three months. We abstracted 
patient characteristics and general medical and ophthalmic history. For each followup visit, VA, ophthalmic exam findings, imaging, and intervention characteristics were recorded for both eyes. If patients received a different treatment during follow-up, data from beyond the change of treatment were not included. Data was integrated in a secured database (Microsoft Office Access Edition 2003).

Visual acuity results were clustered by 2-4, 6-8, 10-12, and 22-24 months after the first intervention, or after the time of diagnosis for the no-treatment group. Snellen or Early Treatment Diabetic Retinopathy Study (ETDRS) VA was converted to logarithm of the minimal angle of resolution (LogMAR). We performed analysis with the Pearson's chisquare test and ANOVA to assess group differences in categorical variables and continuous variables, respectively. We used linear mixed models to calculate and compare the VA change from baseline at each follow-up cluster between the patient groups. Study group type and follow-up time were factors in the model. Age, gender, baseline VA, type of lesion, presence of blood, size of the lesion and cataract surgery were covariates. We used the statistically significant contributing covariates in the final model. Analyses were performed with SPSS Statistics (IBM, Chicago, IL).

Our structured outcome and process assessment comprised of: (A) Selecting the VA in the treated cohorts as the relevant outcome parameter; (B) Comparing the outcome with the comparators: VA outcome in the no-treatment group (technically a historical cohort), the outcome in the other treatment cohorts, and the outcome in randomised controlled trials (RCTs); (C) Correcting for confounding variables; and D) Identifying relevant patient characteristics and process variables that determine outcome, in order to improve daily practice. 


\section{Results}

\section{The retrospective study}

The selection process of patients is shown in Figure 1. A total of 473 patients (473 eyes) were included. Mean age was 78.4 years \pm 7.3 (standard deviation), mean follow-up was $3.2 \pm 2.7$ years. Baseline characteristics are presented in Table 1. Groups differed statistically significant in terms of age, lesion type, presence of blood, and classification of CNV in the fellow eye. The mean number of interventions was 1.9 (range 1-6), 3.7 (19) and 4.3 (1-10) in one year for PDT, bevacizumab and ranibizumab, respectively. A switch to another intervention during the follow-up occurred in 28 (20\%), 17 (13\%) and 33 (32\%) patients in the PDT, bevacizumab and ranibizumab groups, respectively. Fortyone patients in the no-treatment group received photocoagulation and four patients received photocoagulation (juxtafoveal and extrafoveal) before their treatment with PDT. In total four, five, nine and eleven patients had received cataract surgery during the follow-up in the no treatment group, PDT, ranibizumab and bevacizumab groups, respectively.

\section{A. The outcome parameter}

Change in VA in the treated cohorts is shown in Table 2. Visual acuity improved in eyes treated with the anti-VEGFs ranibizumab and bevacizumab in the first months, to slightly taper off in long-term follow-up.

\section{B. The comparators}

We compared VA in treated eyes with VA in the non-treated cohort, where VA declined over time (Table 2). There was no difference between PDT and no treatment. There was a benefit of more than 0.4 LogMAR for the anti-VEGFs over no treatment (at 6 to 24 months). We also compared the study outcomes to outcomes in RCTs and other dailypractice outcome studies (Table 3).

\section{Confounding}

Table 1 shows a difference in the type of CNV lesions between the PDT and anti-VEGF groups. After adjusting for lesion type as a confounder, there was no significant effect of PDT over no treatment, and anti-VEGFs remained equally effective.

\section{Identifying patient and process variables that determine the outcome}

Disease severity is a relevant patient characteristic impacting treatment success. In nAMD, this can be derived from size of the CNV lesion and the presence of blood (Table 1). The appropriateness of diagnosis is an important process variable. In re-examining FAs, evaluating the diagnostics process, we found that a total of 104 patients had not 
received an appropriate diagnosis of $\mathrm{nAMD}$ or did not have an appropriate indication for treatment. Moreover, number of initial injections was lower, and time between injections was longer than in current strategies. It is known that this may worsen the outcome.

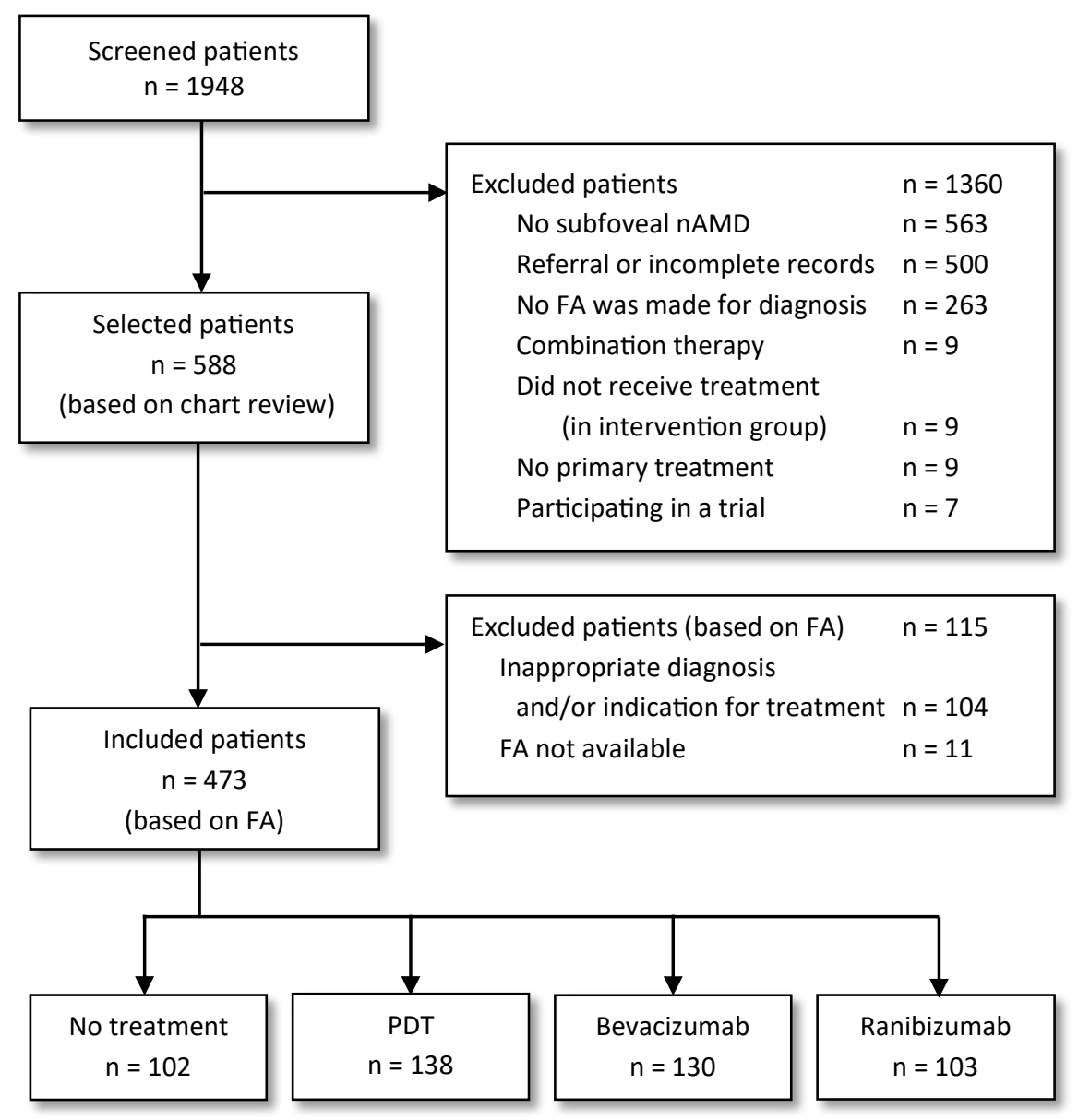

Figure 1. Flow chart of the selection process of patients. $n A M D$, neovascular age-related macular degeneration; $F A$, fluorescein angiography; $n$, number of patients; $P D T$, verteporfin photodynamic therapy. 
Table 1. Baseline characteristics, by study group of patients with subfoveal CNV due to age-related macular degeneration.

\section{Baseline Characteristics}

Female (\%)

Mean age \pm SD

Angiographic lesion subtype*

Predominantly classic (\%)

Minimally classic (\%)

Occult with no classic (\%)

Cannot classify: obscuring lesion (\%)

Cannot classify: photo quality (\%)

Angiographic non-CNV components of lesion*

Elevated blocked hypofluorescence

Blood

PED

Fibrous tissue

Angiographic measurements

Size CNV (disc diameters) $\pm \mathrm{SD}$

Missing size $\mathrm{CNV}$

Size of lesion (disc diameters) $\pm S D$

Angiographic classification of fellow eye $†$

No nAMD (\%)

Early or intermediate nAMD (\%)

Advanced nAMD (\%)

Cannot classify: photo quality (\%)

Visual acuity

Study eye LogMAR \pm SD

Fellow eye LogMAR \pm SD

Best eye $\log M A R \pm S D$
No Treatment $(\mathrm{N}=102)$

$51(50)$

$77.7 \pm 7.8$

40 (39)

11 (11)

46 (45)

5 (5)

1 (1)

36 (35)

6 (6)

$1.3 \pm 0.8$

2

$1.8 \pm 0.8$

11 (11)

40 (39)

49 (48)

2 (2)

$0.82 \pm 0.49$

$0.67 \pm 0.73$

$0.38 \pm 0.33$

$n A M D$, neovascular age-related macular degeneration; $C N V$, choroidal neovascularization; LogMAR, logarithm of the minimal angle of resolution; MPS, macular photocoagulation study; $N$, number of patients; $P E D$, pigment epithelial detachment; $P D T$, verteporfin photodynamic therapy; $S D$, standard deviation. 


\begin{tabular}{|c|c|c|c|}
\hline $\operatorname{PDT}(\mathrm{N}=138)$ & Bevacizumab $(\mathrm{N}=130)$ & Ranibizumab $(\mathrm{N}=103)$ & $\mathrm{p}$-value \\
\hline $79(57)$ & $77(59)$ & $54(52)$ & 0.5 \\
\hline \multirow[t]{2}{*}{$76.9 \pm 7.5$} & $78.6 \pm 6.9$ & $80.8 \pm 6.3$ & $<0.0001$ \\
\hline & & & $<0.0001$ \\
\hline $82(59)$ & $33(25)$ & $49(48)$ & \\
\hline $16(12)$ & $24(19)$ & $10(10)$ & \\
\hline $36(26)$ & $59(45)$ & $36(35)$ & \\
\hline $4(3)$ & $14(11)$ & $6(6)$ & \\
\hline- & - & $2(2)$ & \\
\hline $2(1)$ & $1(1)$ & - & 0.7 \\
\hline $74(54)$ & $58(45)$ & $54(52)$ & 0.02 \\
\hline $8(6)$ & $15(12)$ & $6(6)$ & 0.2 \\
\hline- & $1(1)$ & - & 0.5 \\
\hline $1.4 \pm 0.8$ & $1.2 \pm 0.8$ & $1.2 \pm 0.7$ & 0.3 \\
\hline 1 & & & \\
\hline \multirow[t]{2}{*}{$1.8 \pm 0.8$} & $2.0 \pm 0.8$ & $1.9 \pm 0.8$ & 0.3 \\
\hline & & & $<0.0001$ \\
\hline $22(16)$ & $26(20)$ & $23(22)$ & \\
\hline $41(30)$ & $51(39)$ & $30(29)$ & \\
\hline $64(46)$ & $51(39)$ & $35(34)$ & \\
\hline $11(8)$ & $2(2)$ & $15(15)$ & \\
\hline $0.73 \pm 0.37$ & $0.73 \pm 0.41$ & $0.77 \pm 0.48$ & 0.3 \\
\hline $0.64 \pm 0.75$ & $0.70 \pm 0.82$ & $0.64 \pm 0.76$ & 0.9 \\
\hline $0.36 \pm 0.35$ & $0.35 \pm 0.32$ & $0.38 \pm 0.40$ & 0.9 \\
\hline
\end{tabular}

* Definitions based on classification of the Macular Photocoagulation Study (MPS). ${ }^{2}$

† Definitions based on classification of the Age-Related Eye Disease Study Research Group (AREDS) study. 
Table 2. Regression coefficients of the mean changes in visual acuity by study group.

\begin{tabular}{lcccccc}
\hline \multirow{2}{*}{$\begin{array}{l}\text { Follow-up } \\
\text { (months) }\end{array}$} & No Treatment* & PDT (p-value) & Bevacizumab (p-value) & Ranibizumab (p-value) \\
\cline { 2 - 7 } & 0.27 & 0.18 & $(0.2)$ & -0.01 & $(<0.0001)$ & $-0.12 \quad(<0.0001)$ \\
$6-8$ & 0.38 & 0.34 & $(0.6)$ & 0.002 & $(<0.0001)$ & $-0.10 \quad(<0.0001)$ \\
$10-12$ & 0.54 & 0.48 & $(0.5)$ & 0.09 & $(<0.0001)$ & $0.07 \quad(<0.0001)$ \\
$22-24$ & 0.69 & 0.61 & $(0.5)$ & 0.12 & $(<0.0001)$ & $0.13 \quad(<0.0001)$ \\
\hline
\end{tabular}

LogMAR, logarithm of the minimal angle of resolution; PDT, Verteporfin photodynamic therapy; VA, visual acuity.

* Reference group.

Note: a positive LogMAR change indicates a decline in visual acuity.

Table 3. Visual acuity in patients with neovascular age-related macular degeneration without treatment in daily practice, or after PDT, bevacizumab or ranibizumab in daily practice and in patients from clinical trials.

\begin{tabular}{|c|c|c|c|c|c|}
\hline \multirow[b]{3}{*}{ Regimen } & \multicolumn{4}{|c|}{ VA Change (LogMAR) at 12 Months } & \multirow{3}{*}{$\begin{array}{l}\text { Literature } \\
\text { Reference }\end{array}$} \\
\hline & \multicolumn{2}{|c|}{ From Baseline } & \multicolumn{2}{|c|}{ Versus Control Group } & \\
\hline & This Study & Literature & This Study* & Literature & \\
\hline No Treatment & 0.54 & 0.2 to 0.4 & - & - & $3,29,34,35$ \\
\hline PDT & 0.48 & 0.2 to 0.3 & -0.06 & -0.14 & $3,25,34,35$ \\
\hline Bevacizumab & 0.09 & -0.1 to -0.16 & -0.45 & $-0.33 \dagger$ & $5,36,37$ \\
\hline Ranibizumab & 0.07 & -0.1 to -0.2 & -0.47 & -0.35 to -0.41 & $5,25,29,37$ \\
\hline PIER study & & 0.04 & & & 27 \\
\hline SAILOR study & & -0.04 & & & 38 \\
\hline $\begin{array}{l}\text { Anti-VEGF } \\
\text { outcome studies }\end{array}$ & & -0.1 to -0.02 & & & $14,39-51$ \\
\hline
\end{tabular}

* Compared to the no-treatment cohort.

† Compared to 'standard care' in the ABC trial.

Note: a negative LogMAR change indicates an improvement in visual acuity.

LogMAR, logarithm of the minimal angle of resolution; PDT, Verteporfin Photodynamic Therapy; VA, visual acuity. 


\section{Discussion}

Daily-practice data analyses are fundamental in improving the value of care. As put forward by M.E. Porter:4 "Since value is defined as outcomes relative to costs, it encompasses efficiency. Cost reduction without regard to the outcomes achieved is dangerous and self-defeating, leading to false "savings" and potentially limiting effective care." We ran a process and outcome analysis for the treatment of nAMD in daily practice, showing that such analysis involves several steps: selecting the appropriate outcome parameter; comparing this outcome with the appropriate comparator(s); correcting for confounders; and, finally, Identifying relevant process variables that are suitable for improvement in a feed-back loop to improve daily practice and the value of care.

\section{A. Selecting the relevant outcome parameter}

Measurements, expected to improve, or adverse events, can be relevant outcomes. Examples in nAMD are VA and quality-of-life. Relevant outcomes are directly relevant to the patient and a focus for improvement through treatment. For external comparison purposes, it is useful if the outcome was used in trials.

Intermediate outcome parameters can also be relevant. These reside in the causal pathway from treatment to outcome: improving an intermediate outcome will improve a final outcome. In the case of $\mathrm{nAMD}$, the presence of CNV on FA, edema detected on OCT, or blood detected on fundoscopy can be regarded intermediate outcome variables.

In our study, we used VA as the relevant outcome, as it greatly determines quality-oflife and treatment decisions. It is frequently recorded in medical visits. It also allows for comparison with RCT results. OCT was not in clinical use during the follow-up of the no-treatment cohort. FA was not commonly repeated in the time-periods before the introduction of effective treatment, and after OCT was introduced. Quality-of-life was not useful in this retrospective analysis, as it is rarely measured in daily practice.

\section{B. Comparing the outcome parameter with a comparator and selecting the right comparator}

The outcome needs a comparator outcome, to identify outcome differences which may indicate opportunities to improve processes. Comparators can be selected, depending on the disease, the intervention and the research question. We focused on assessing the effectiveness of treatment in daily practice, and emphasise the need for a comparison with untreated patients for this assessment. We address several approaches and show the methodological pitfalls. We show that the choice of the comparator can lead to different results and conclusions. 


\section{Comparing with an untreated historical cohort}

To validly assess intervention effect, it is important to select an untreated comparison cohort. The outcome in an untreated cohort must reflect the treated cohort's theoretical natural history, had it not received treatment. Selecting an untreated historical cohort from the same setting (clinic) probably reflects the characteristics of treated nAMD patients well. As patient characteristics may change over time, it is helpful to select a cohort from the not too distant past.

Our no-treatment group was from the time-period 1992 to 1997. We assumed that this group reflected the treated patients' theoretical natural history, had they not received treatment. However, even so, patient characteristics showed a difference in age, lesion subtype, presence of blood, and fellow eye status (Table 1). These may influence the outcome, as they are prognostic factors for the natural history of nAMD. Importantly, there was no difference in mean VA between groups. As expected, VA decreased over time in the no-treatment group (Table 2). There was no difference in outcome between PDT and no treatment. There was an obvious difference in outcome between antiVEGFs compared to no treatment: a clinically meaningful benefit of 0.4 LogMAR at 10-12 months.

The lack of outcome difference between PDT and no treatment could be due to a lower prevalence of classic CNV in the no-treatment group. This implies that the no-treatment group may not reflect the theoretical PDT groups' natural history, without PDT. Generally, untreated and PDT-treated classic CNVs have worse outcomes than untreated occult CNVs. However, even after adjusting for baseline characteristics like CNV type and baseline VA, there was no difference in outcome between PDT and no treatment, and the anti-VEGFs still had statistically significant better outcomes compared to the notreatment group at 10-12 months.

\section{Comparing with an untreated cohort from the same time period}

Comparing a treated group with an untreated group from the same time period may seem attractive. However, this incurs a high risk of confounding by indication, potentially inducing important interpretation errors. In nAMD, patients with severe macular hemorrhage may not receive any treatment. Comparing an anti-VEGF treated group with these patients will not produce meaningful results, as the no-treatment group does not reflect the hypothetical natural history of the anti-VEGF group. In other instances, when a favorable outcome can be expected without treatment, patients may go untreated. In this case, a treated group, with more severe disease at baseline, may have poorer outcomes than the untreated group. In our study, we decided not to compare with an untreated group from the same time period. 


\section{Comparing with other interventions}

Rather than comparing with an untreated group, outcomes can be compared between treatments. An advantage is that the indication is more likely to be similar. However, in nAMD, PDT and anti-VEGF have different indications. In comparing anti-VEGFs to each other and to PDT, we therefore adjusted for baseline differences in, for example, CNV type. With the PDT group as a reference group, we found that bevacizumab and ranibizumab had better outcomes. There was no difference in outcome between bevacizumab and ranibizumab (Table 2).

\section{Comparing with clinical trials}

Comparing the outcome from daily practice with the outcome from RCTs may yield a difference and thus indications for improving daily-practice treatment outcome. Differences between these data sources are important. The outcome (VA) may not be measured as accurately in daily practice as in RCTs. However, such measurements do determine the decision-making by clinicians. In RCTs, patients are generally selected with strict criteria. This may yield a population in which the diagnosis is more thoroughly established, which could lead to a better treatment outcome. Inclusion criteria may select patients that are more sensitive for improvement with a specific intervention, or with a more severe disease status. Follow-up can be more frequent, with better equipment and higher-quality assessment of re-treatment criteria. Compliance-enhancing strategies could have been implemented.

A first step is to compare the untreated daily practice group with the RCT's placebo group (Table 3). A difference between the groups' prognosis may be suggested. The VA change at twelve months in the no-treatment group in our study was worse than in RCTs, implying that prognosis was worse in our patients.

The next comparison can be the daily-practice treatment outcome compared to the outcome in RCTs. For PDT, the daily-practice outcome appeared worse than in RCTs (Table 3). However, offsetting the treatment outcomes to the respective untreated groups (yielding the 'effect size') actually yields VA benefits due to PDT in both daily practice and RCTs. However, outcomes were still worse in daily practice than in RCTs and not statically different from the natural history. This is an indication to further examine differences in patient and treatment characteristics, to explain differences in outcome. This could reveal possibilities to improve the patient selection or the process of the intervention.

For the anti-VEGFs results, interpretation requires similar attention. The outcomes, from baseline, in daily practice seemed worse than in the intervention arms in RCTs (Table 3). However, the effect size (outcome versus control group) is greater in our study than in 
RCTs. Relevant prognostic factors were taken into account. Thus, effectiveness of antiVEGF appeared as good as in RCTs. The outcomes, versus baseline, of our study seem more in line with the treated patients in the PIER and SAILOR study, which showed a lower gain in VA from baseline compared to earlier RCTs. In these studies, patients received a reduced number of treatments due to a planned longer follow-up of three months between two injections. A reduced number of injections by itself will not lead to a lower VA if monthly follow-up takes place and injections are given pro re nata based on adequate criteria. ${ }^{5-8}$ However, to achieve optimum outcome, a three months follow-up period is too long. 9

\section{Comparing with other daily-practice outcome studies}

Our daily-practice results are consistent with other retrospective outcome studies, which found VA to stabilise or decline after one year of anti-VEGF treatment.10-12 Tao et al. found a VA decline after two years. ${ }^{13}$ In contrast, a majority of outcome studies found a VA improvement of at least -o.1 logMAR after one year treated with anti-VEGFs (Table 3). A very large outcome study in over 11,000 patients showed VA stabilisation over a period of 3 years with a yearly median number of 4-5 injections and 8.2 to 9.2 outpatient visits. ${ }^{14}$

\section{Correcting outcome comparisons for confounding variables}

In comparing outcomes between cohorts, it is very important to consider what caused the observed difference, and this cause needs to be found. For example, one could question whether a difference in VA after treatment with PDT or anti-VEGF is really caused by the difference in treatment. For this question, confounding variables to be taken into account. Relevant variables are patient characteristics that are prognostic factors, or factors that modify the effect of treatment. In comparing PDT and anti-VEGF, we needed to adjust for CNV lesion type, since PDT was not indicated for every type: we observed a difference in CNV lesion types between PDT and anti-VEGF groups (Table 1). Moreover, lesion type determines the prognosis. ${ }^{15-17}$ We found that, after adjusting for lesion type, occult or classic CNV, there was no effect of PDT.

Comparing the difference in outcome between daily-practice ranibizumab and untreated patients to the difference between RCT-ranibizumab and placebo, effect-modifying variables need to be taken into account. Lesion type and size can be effect-modifying variables. Effect on VA, treatment versus sham, is greater in classic CNV than occult CNV and in smaller lesion than larger ones. ${ }^{16}$ If effect modification is considered, a stratified analysis based on levels of the effect-modifying variable is needed to see if the effect size differs between different levels of the variable. Lesion as effect-modifier was taken into account to assess the difference in outcome between PDT and anti-VEGF. 
A special case of confounding is confounding by indication. Comparing patients treated with PDT or an anti-VEGF to untreated patients in the same period, a difference in outcome can be due to confounding by indication, even after taking other confounding variables into account. Ophthalmologists consider patients eligible for treatment based on expected prognosis or treatment effect. Therefore, a priori, the outcome is different between treated and untreated patients from the same period. Comparing two treatments with different selection criteria may induce similar confounding. In our study, after taking confounding factors (lesion type) into account, there still was a difference in outcome between PDT and anti-VEGFs. To a high degree of validity, anti-VEGFs were more effective in daily practice than PDT, unless the difference is due to confounding variables that were not measured, or are difficult to measure, such as confounding by indication.

\section{Identifying the relevant patient characteristics and process variables that determine the outcome}

The ultimate goal of outcome and process analysis is to identify factors which need improvement in the process of patient selection, diagnosis and selection. In the previous sections, the relevant factors that determine outcome have been outlined. Methods to determine the effectiveness of the treatments, and the effect sizes, corrected for confounding, and major pitfalls, have been outlined. In this important additional step, several process factors, variables that commonly have impact on the outcome, are regarded: severity of the disease at first presentation; physician-dependent process variables; and patient-dependent process variables.

\section{Severity of the disease at baseline}

Severity of the disease determines the prognosis, type of intervention indicated, and the outcome. We identified several severity factors for nAMD. These included the lesion type; lesion size and presence of blood in the lesion (see Table 1). These variables determine the prognosis without treatment, and the effect of treatment with e.g. PDT and antiVEGF. ${ }^{15}{ }^{16}$ If the focus is improvement of outcome of nAMD care, earlier access to an ophthalmologist is a focus for improvement: Literature indicates that delay in the access of the patient to a general practitioner or late referral to an ophthalmologist occurs and influences the outcome of nAMD treatment. ${ }^{18}$ Earlier referral may lead to a lower severity of the disease at baseline.

\section{Physician-dependent process variables}

Addressing physician-dependent process variables includes assessment of whether the diagnosis was correct, whether the indication for a treatment was correct, and whether the treatment or intervention was correctly timed and conducted. The diagnosis and the indication for treatment (subfoveal CNV due to nAMD), was re-assessed in our 
study by retina specialists. In 104 patients, diagnosis or indication for treatment was not appropriate. It is known that classification of CNV type or assessment of retreatment criteria differ between ophthalmologists. ${ }^{19}, 20$ Improving the rate of correct diagnosis and indication will improve outcomes as treatment is unlikely to be effective without the presence of $\mathrm{CNV}$. In addition, literature indicates that the timing of the intervention may impact the outcome..$^{21,22}$

Sometimes, treatments may not be in accordance with the "state-of-the-art". Improving care in this regard requires a definition of the state-of-the art, provided by guidelines or clinical trials. In our study, PDT was given according to the TAP protocol with a follow-up period of three months and a maximum of four PDT treatments per year. ${ }^{3}$ Early after the introduction of anti-VEGF treatments, some repeated treatments with anti-VEGF were given with longer intervals as compared to early RCTs. In the RCTs, monthly treatments or

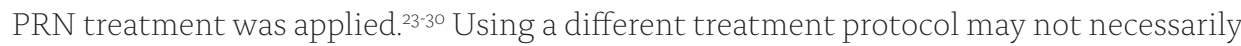
worsen outcomes. Ophthalmologists may individualise treatment to optimise outcomes, and they may take into account a strategy preferred by the patient, or may prioritise avoiding adverse events over other potential benefits of treatment. However, we now know that three-month intervals between anti-VEGF injections of bevacizumab as a standard is too long and adversely affects the outcome. ${ }^{27}$

\section{Patient-dependent process variables}

Patient-dependent process variables concern timely reporting of symptoms, and adherence to prescribed medication, to follow-up appointments and to life-style advice. We did not specifically address these in our study, since it was based on a medical record review. For example, no questions were addressed regarding patient delay. In nAMD, timely reporting of symptom recurrence and timely follow-up are known to be important patient related-process variables that impact the outcome. ${ }^{21,31,32}$ 


\section{Conclusions}

This article contributes to the opportunities for an ophthalmologist to participate in data analysis and discussions in the era of value-based health care. These discussions are inevitable since value-based health care is seen as a "breakthrough that will change the face of medicine, the goal of which is to lower health care costs and improve quality and outcomes."33

As supported by this study, in the case of nAMD, preventing patient and doctor delay, improving the appropriateness of diagnosis and indication for treatment, using antiVEGFs and not using PDT, and addressing the frequency of follow-up and frequency, anti-VEGFs will improve the value for patients.

\section{Acknowledgements}

We thank the coordinators and co-workers for their help at the departments of ophthalmology of the VU University Medical Center Amsterdam, Admiraal de Ruyter Hospital, ZorgSaam Zeeuws-Vlaanderen and Catharina Hospital Eindhoven. We thank dr. E.C. La Heij (retina specialist), dr. F. Lion (retina specialist) and prof. F. Hendrikse (retina specialist) for their major contribution to evaluate FAs. 


\section{References}

1. Risk factors associated with age-related macular degeneration. A case-control study in the age-related eye disease study: Age-Related Eye Disease Study Report Number 3. Ophthalmology 2000;107:22242232.

2. Subfoveal neovascular lesions in age-related macular degeneration. Guidelines for evaluation and treatment in the macular photocoagulation study. Macular Photocoagulation Study Group. Arch Ophthalmol 1991;109:1242-1257.

3. Bressler NM. Photodynamic therapy of subfoveal choroidal neovascularization in age- related macular degeneration with verteporfin: One-year results of 2 randomized clinical trials - TAP report 1. Archives of Ophthalmology 1999;117:1329-1345.

4. Porter ME. What is value in health care? N Engl J Med 2010;363:2477-2481.

5. Chakravarthy U, Harding SP, Rogers CA, et al. Ranibizumab versus bevacizumab to treat neovascular age-related macular degeneration: one-year findings from the IVAN randomized trial. Ophthalmology 2012;119:1399-1411.

6. Lalwani GA, Rosenfeld PJ, Fung AE, et al. A variable-dosing regimen with intravitreal ranibizumab for neovascular age-related macular degeneration: year 2 of the PrONTO Study. Am J Ophthalmol 2009;148:43-58 e41.

7. Martin DF, Maguire MG, Fine SL, et al. Ranibizumab and bevacizumab for treatment of neovascular age-related macular degeneration: two-year results. Ophthalmology 2012;119:1388-1398.

8. Schmidt-Erfurth U, Eldem B, Guymer R, et al. Efficacy and safety of monthly versus quarterly ranibizumab treatment in neovascular age-related macular degeneration: the EXCITE study. Ophthalmology 2011;118:831-839.

9. Simader C, Bolz M, Ritter M, Golbaz I, Ahlers C, Schmidt-Erfurth U. Ranibizumab on Visual Acuity, Retinal Thickness and Retinal Morphology When Administered Monthly vs. Quaterly With Different Dosing Regimens in the Excite Trial. ARVO. Vienna; 2009.

10. Bloch SB, la Cour M, Sander B, et al. Predictors of 1-year visual outcome in neovascular age-related macular degeneration following intravitreal ranibizumab treatment. Acta Ophthalmol 2013;91:42-47.

11. Cohen SY, Dubois L, Tadayoni R, et al. Results of one-year's treatment with ranibizumab for exudative age-related macular degeneration in a clinical setting. Am J Ophthalmol 2009;148:409-413.

12. Fong DS, Custis P, Howes J, Hsu JW. Intravitreal bevacizumab and ranibizumab for age-related macular degeneration a multicenter, retrospective study. Ophthalmology 2010;117:298-302.

13. Tao Y, Libondi T, Jonas JB. Long-term follow-up after multiple intravitreal bevacizumab injections for exudative age-related macular degeneration. Journal of ocular pharmacology and therapeutics: the official journal of the Association for Ocular Pharmacology and Therapeutics 2010;26:79-83.

14. Tufail A, Xing W, Johnston R, et al. The neovascular age-related macular degeneration database: multicenter study of 92976 ranibizumab injections: report 1: visual acuity. Ophthalmology 2014;121:1092-1101.

15. Blinder KJ, Bradley S, Bressler NM, et al. Effect of lesion size, visual acuity, and lesion composition on visual acuity change with and without verteporfin therapy for choroidal neovascularization secondary to age-related macular degeneration: TAP and VIP report no. 1. Am J Ophthalmol 2003;136:407-418.

16. Boyer DS, Antoszyk AN, Awh CC, Bhisitkul RB, Shapiro H, Acharya NR. Subgroup analysis of the MARINA study of ranibizumab in neovascular age-related macular degeneration. Ophthalmology 2007;114:246-252. 
17. Elshout M, Webers CA, van der Reis MI, de Jong-Hesse Y, Schouten JS. Tracing the natural course of visual acuity and quality of life in neovascular age-related macular degeneration: a systematic review and quality of life study. BMC ophthalmology 2017;17:120.

18. Haddad WM, Seres A, Coscas G, Soubrane G. Presentation delay in patients affected with exudative age-related macular degeneration. Graefes Arch Clin Exp Ophthalmol 2002;240:31-34.

19. Holz FG, Jorzik J, Schutt F, Flach U, Unnebrink K. Agreement among ophthalmologists in evaluating fluorescein angiograms in patients with neovascular age-related macular degeneration for photodynamic therapy eligibility (FLAP-study). Ophthalmology 2003;110:400-405.

20. Muni RH, Altaweel M, Tennant M, Weaver B, Kertes PJ. Agreement among Canadian retina specialists in the determination of treatment eligibility for photodynamic therapy in age-related macular degeneration. Retina 2008;28:1421-1426.

21. Lim JH, Wickremasinghe SS, Xie J, et al. Delay to treatment and visual outcomes in patients treated with anti-vascular endothelial growth factor for age-related macular degeneration. Am J Ophthalmol 2012;153:678-686, 686 e671-672.

22. Muether PS, Hoerster R, Hermann MM, Kirchhof B, Fauser S. Long-term effects of ranibizumab treatment delay in neovascular age-related macular degeneration. Graefes Arch Clin Exp Ophthalmol 2013;251:453-458.

23. Avery RL, Pieramici DJ, Rabena MD, Castellarin AA, Nasir MA, Giust MJ. Intravitreal bevacizumab (Avastin) for neovascular age-related macular degeneration. Ophthalmology 2006;113:363-372 e365

24. Bashshur ZF, Haddad ZA, Schakal A, Jaafar RF, Saab M, Noureddin BN. Intravitreal bevacizumab for treatment of neovascular age-related macular degeneration: a one-year prospective study. Am J Ophthalmol 2008;145:249-256.

25. Brown DM, Kaiser PK, Michels $M$, et al. Ranibizumab versus verteporfin for neovascular age-related macular degeneration. N Engl J Med 2006;355:1432-1444.

26. Emerson MV, Lauer AK, Flaxel CJ, et al. Intravitreal bevacizumab (Avastin) treatment of neovascular age-related macular degeneration. Retina 2007;27:439-444.

27. Regillo CD, Brown DM, Abraham P, et al. Randomized, double-masked, sham-controlled trial of ranibizumab for neovascular age-related macular degeneration: PIER Study year 1. Am J Ophthalmol 2008;145:239-248.

28. Rich RM, Rosenfeld PJ, Puliafito CA, et al. Short-term safety and efficacy of intravitreal bevacizumab (Avastin) for neovascular age-related macular degeneration. Retina 2006;26:495-511.

29. Rosenfeld PJ, Brown DM, Heier JS, et al. Ranibizumab for neovascular age-related macular degeneration. N Engl J Med 2006;355:1419-1431.

30. Spaide RF, Laud K, Fine HF, et al. Intravitreal bevacizumab treatment of choroidal neovascularization secondary to age-related macular degeneration. Retina 2006;26:383-390.

31. Real JP, Granero GE, De Santis MO, et al. Rate of vision loss in neovascular age-related macular degeneration explored. Graefes Arch Clin Exp Ophthalmol 2015;253:1859-1865.

32. Takahashi H, Ohkubo Y, Sato A, et al. RELATIONSHIP BETWEEN VISUAL PROGNOSIS AND DELAY OF INTRAVITREAL INJECTION OF RANIBIZUMAB WHEN TREATING AGE-RELATED MACULAR DEGENERATION. Retina 2015;35:1331-1338.

33. Cosgrove T. Value-Based Health Care is Inevitable and That's Good. Harvard Business Review 2013.

34. Arnold J, Barbezetto I, Birngruber R, et al. Verteporfin therapy of subfoveal choroidal neovascularization in age-related macular degeneration: Two-year results of a randomized clinical trial including lesions with occult with no classic choroidal neovascularization - Verteporfin in photodynamic therapy report 2. American Journal of Ophthalmology 2001;131:541-560. 
35. Kaiser PK. Verteporfin PDT for subfoveal occult CNV in AMD: two-year results of a randomized trial. Curr Med Res Opin 2009;25:1853-1860.

36. Tufail A, Patel PJ, Egan C, et al. Bevacizumab for neovascular age related macular degeneration (ABC Trial): multicentre randomised double masked study. Bmj 2010;340:c2459.

37. Martin DF, Maguire MG, Ying GS, Grunwald JE, Fine SL, Jaffe GJ. Ranibizumab and bevacizumab for neovascular age-related macular degeneration. N Engl J Med 2011;364:1897-1908.

38. Boyer DS, Heier JS, Brown DM, Francom SF, Ianchulev T, Rubio RG. A Phase IIIb study to evaluate the safety of ranibizumab in subjects with neovascular age-related macular degeneration. Ophthalmology 2009;116:1731-1739.

39. Arevalo JF, Sanchez JG, Wu L, et al. Intravitreal bevacizumab for subfoveal choroidal neovascularization in age-related macular degeneration at twenty-four months: the Pan-American Collaborative Retina Study. Ophthalmology 2010;117:1974-1981, 1981 e1971.

40. Bellerive C, Cinq-Mars B, Lalonde G, et al. Bevacizumab and ranibizumab for neovascular age-related macular degeneration: a treatment approach based on individual patient needs. Can J Ophthalmol 2012;47:165-169

41. Costagliola $\mathrm{C}$, Romano $\mathrm{M}$, Corte $\mathrm{MD}$, et al. Intravitreal bevacizumab for treatment-naive patients with subfoveal occult choroidal neovascularization secondary to age-related macular degeneration: a 12-month follow-up study. Retina 2009;29:1227-1234.

42. Ernst BJ, Barkmeier AJ, Akduman L. Optical coherence tomography-based intravitreal ranibizumab (Lucentis) for neovascular age-related macular degeneration. Int Ophthalmol 2010;30:267-270.

43. Fung AE, Lalwani GA, Rosenfeld PJ, et al. An optical coherence tomography-guided, variable dosing regimen with intravitreal ranibizumab (Lucentis) for neovascular age-related macular degeneration. Am J Ophthalmol 2007;143:566-583.

44. Gamulescu MA, Radeck V, Lustinger B, Fink B, Helbig H. Bevacizumab versus ranibizumab in the treatment of exudative age-related macular degeneration. Int Ophthalmol 2010;30:261-266.

45. Katz G, Giavedoni L, Muni R, et al. Effectiveness at 1 year of monthly versus variable-dosing intravitreal ranibizumab in the treatment of choroidal neovascularization secondary to age-related macular degeneration. Retina 2012;32:293-298.

46. Luu ST, Gray T, Warrier SK, et al. Retrospective study of an as required dosing regimen of intravitreal bevacizumab in neovascular age-related macular degeneration in an Australian population. Clinical \& experimental ophthalmology 2010;38:659-663.

47. Mekjavic PJ, Kraut A, Urbancic M, Lenassi E, Hawlina M. Efficacy of 12-month treatment of neovascular age-related macular degeneration with intravitreal bevacizumab based on individually determined injection strategies after three consecutive monthly injections. Acta Ophthalmol 2011;89:647-653.

48. Michalova K, Wickremasinghe SS, Tan TH, et al. Ranibizumab treatment for neovascular age-related macular degeneration: from randomized trials to clinical practice. Eye (Lond) 2009;23:1633-1640.

49. Querques G, Azrya S, Martinelli D, et al. Ranibizumab for exudative age-related macular degeneration: 24-month outcomes from a single-centre institutional setting. Br J Ophthalmol 2010;94:292-296.

50. Rothenbuehler SP, Waeber D, Brinkmann CK, Wolf S, Wolf-Schnurrbusch UEK. Effects of Ranibizumab in Patients with Subfoveal Choroidal Neovascularization Attributable to Age-related Macular Degeneration. American Journal of Ophthalmology 2009;147:831-837.

51. Shienbaum G, Gupta OP, Fecarotta C, Patel AH, Kaiser RS, Regillo CD. Bevacizumab for neovascular age-related macular degeneration using a treat-and-extend regimen: Clinical and economic impact. American Journal of Ophthalmology 2012;153:468-473. 

4.
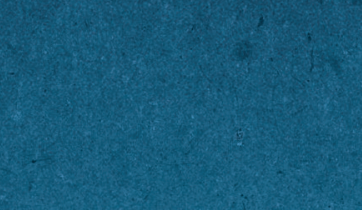

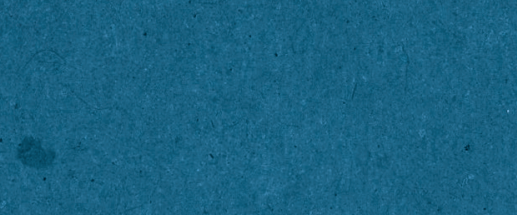
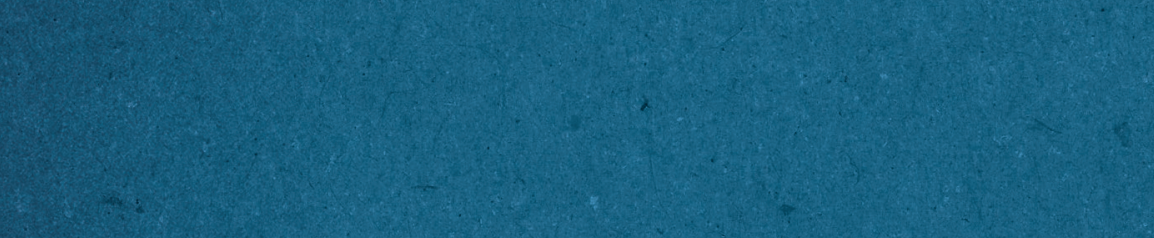

8

$y 3$

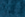




\section{Chapter 8}

General Discussion 


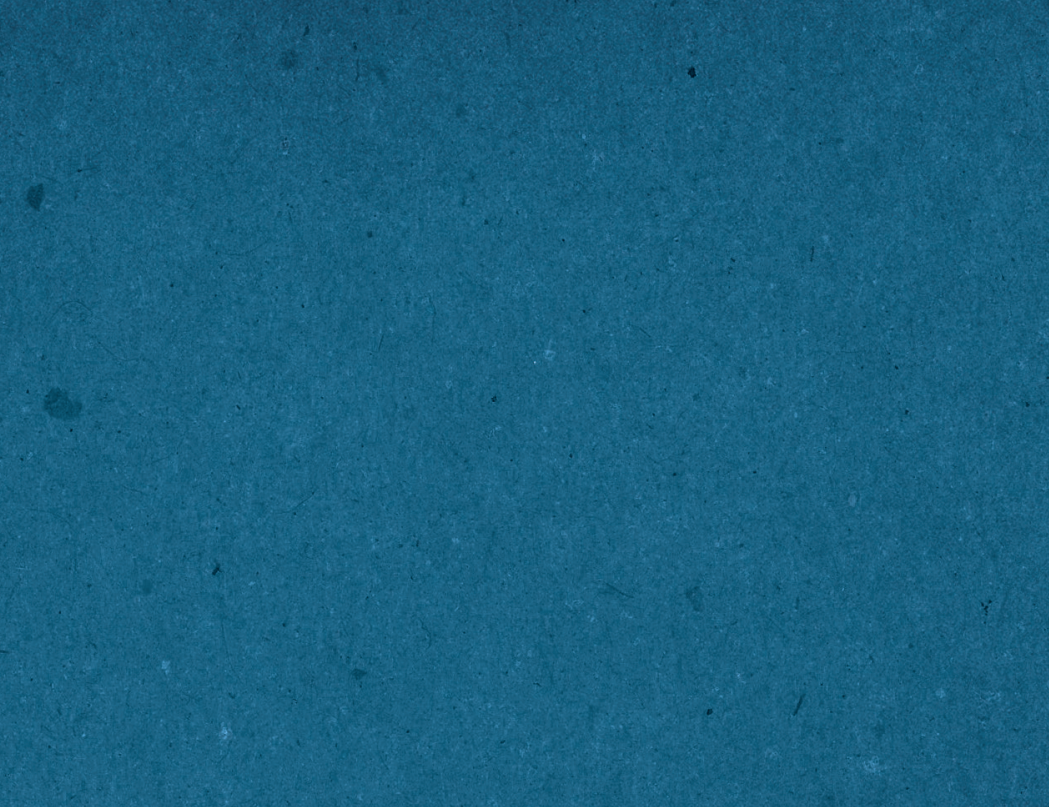




\section{General Discussion}

New and costly treatments are continually emerging. Their potential for clinical application (their "patient value") and their financial value are both addressed from a value-based health care perspective. How should we approach this? How do we balance an improvement in outcome with increasing costs in daily practice? These questions need answers at several stages of deciding on the treatments' value for patients, the reimbursement of treatments and their use in daily practice.

A first step to evaluate patient value is to decide whether the disease, if left untreated, confers a poor outcome or whether it has little impact or only a temporary impact on quality of life. Little value can be gained in short-lived self-limiting diseases with little impact on quality of life. Some have argued not to reimburse treatments for self-limiting diseases with a low impact on quality of life. In this thesis, it was shown that neovascular age-related macular degeneration (nAMD) has a poor prognosis with severe loss of visual acuity within a short period of time when left untreated. Untreated, the loss of visual acuity causes a chronic loss of quality of life. We traced the natural course of visual acuity and quality of life in $\mathrm{nAMD}$ on a meta-level. As was known previously from clinical trials and clinical practice, the decline in visual acuity develops rapidly initially, to gradually taper off as disease duration progresses. Because quality of life is highly dependent on visual acuity in the better-seeing eye (the "fellow eye"), the loss in quality of life follows a different curve, which is highly influenced by the increasing prevalence over time of $\mathrm{nAMD}$ in the better-seeing eyes.

An important recent insight is that the decline in visual acuity may not vary as much between studies as might be understood at first glance from the various trials and natural history studies. In the non-linear declining trend of the visual acuity, the average visual acuity at the start of the prognostic study in combination with the average disease duration, predicts the decline in visual acuity. Correcting for an assumed difference in disease duration between studies, the non-linear change in visual acuity then appears remarkably similar between studies. Studies with the better visual acuity show the change in early patients and studies with a lower visual acuity show the change at a late stage of the disease. Thus, we could process this pattern with a custom meta-analysis in the natural history study combining the change in visual acuity from studies at early and late stages to show the complete change in visual acuity from the start of the study until end stage. 
Several important points can be derived from this analysis. It confirms that the loss of visual acuity in occult neovascular lesions (Type 1 choroidal neovascular lesions) generally progresses at a slower pace than in minimally classic and predominantly classic lesions (Type 2 choroidal neovascular lesions), with predominantly classic lesions conferring the most rapid loss in visual acuity.

The natural progression study adds to the current body of evidence as it not only combines a large number of no-treatment study arms into one analysis, but also because of the additional modelling applied to the data. Modelling was used to address shortcomings in the available data. One of these shortcomings is that the progression of visual acuity was studied, but not the progression of quality of life. Another important shortcoming was that only disease progression of single study eyes was studied and not the occurrence of nAMD and its progression in fellow eyes. Since the fellow eye is likely to have a better visual acuity but may also develop nAMD, this better eye predominantly determines the quality of life of the patient. In this thesis, the natural course of the quality of life of patients, not eyes, was modelled.

As we gain more insight in the prognostic factors of $\mathrm{nAMD}$, these factors can be incorporated in the existing natural progression model to tailor the prediction of the prognosis for an individual patient. Such specific estimates at the patient level could further guide decision-making at the patient level, beyond mean values that are relevant at a population level.

In an effort to gain the highest value for the patient, any intervention, even with a small effect, will be of relevance. Several interventions now exist for nAMD that change the natural course fundamentally, leading to an improvement of visual acuity and quality of life instead of the natural course of a declining visual acuity. These new treatments are costly, but they may be cost-effective.

As a principle, treatments with proven efficacy should be eligible for use in daily practice. However, costs may limit its use if there is no reimbursement through government programmes or health insurance. Governments and health insurance companies could base policies for reimbursement on cost-effectiveness studies. It is known that cost-effectiveness studies may lack quality as required by current state-of-the-art methodology.2, 3 Very few cost-effectiveness studies can be compared due to the wide variety of possible inputs, and therefore standardisation along basic quality parameters is needed.4, 5 We investigated the quality of the current cost-effectiveness studies for the treatment of nAMD. The majority of these studies lacked quality and may therefore severely bias health policy decisions. Seventy per cent of studies did not comply with 
basic quality criteria according to current standards. Moreover, two factors that strongly determine the outcome of a nAMD cost-effectiveness study, a sufficiently long time horizon and the costs of blindness, were not taken into account in more than ninety per cent of the studies.

One fundamental issue in nAMD was also lacking in cost-effectiveness studies and this needs to be discussed in more detail. nAMD is a bilateral disease and eventually affects best visual acuity and quality of life only after the fellow eye is affected, as we have shown in chapter 2. However, costs are incurred from the moment only the first eye with the worst visual acuity is affected. This should be taken into account in cost-effectiveness models. Only a small minority of current cost-effectiveness analyses incorporate this important factor. The other current cost-effectiveness studies, which do not take the bilateral nature of the disease into account, are unreliable, as the outcome measure quality of life, depends on the disease state of the better, mostly untreated eye. However, this better eye is not included in most analyses. Fortunately, more recent analyses have started to either use two-eye models, or explicitly acknowledge that using a one-eye analysis is an important shortcoming. A reason for using only one-eye models could be the added complexity of building a two-eye model. It can be expected that with increasingly open-source study data and analyses, the scientific community will soon build and share uniform two-eye models.

We have developed such a two-eye model and used it to study the cost-utility of aflibercept for the treatment of nAMD compared to bevacizumab and ranibizumab and the influence of model parameters on the results. It is an example model as it incorporates a two-eye model analysis, various time horizons, cost of blindness, and uncertainties such as interpersonal variation in disease progression and treatment response. It is flexible enough to be translated to various other eye diseases and treatment settings, especially since it was based on an earlier glaucoma disease progression model. ${ }^{6}$ It confirmed the influence of several model parameters lacking in other studies on the results of the analysis.

Moreover, this model gives a better basis for reimbursement decisions. It shows that bevacizumab is cost-effective over no treatment as expected, and that aflibercept is cost-effective over ranibizumab. It thus gives a good basis to treat nAMD patients and reimburse these treatments. However, if the treatment needs to start with the expensive anti-VEGFs, total costs are still high. In the Netherlands in 2015, 285,000 injections were given. This would amount to a total cost of about $€ 250$ million for the medications alone if only expensive anti-VEGFs were given. The Dutch guidelines for the treatment of nAMD state that bevacizumab is the treatment of first choice and that treatment should be changed in case bevacizumab is not fully effective. This approach optimises the cost-effectiveness and outcome of treatment. 
The balance between outcome and costs at a patient level in daily practice strongly depends on the decisions made for individual patients and these involve the decisions when to start treatment, when to change treatment and when to stop treatment.

The indication to start the treatment can be based on the inclusion criteria of randomised controlled trials (RCT). However, strict application of these criteria may limit the usefulness of the treatment for many patients. In $\mathrm{nAMD}$, the indication to treat will, in daily practice, be based on the obligatory presence of a (subfoveal) choroidal neovascularisation. Contraindications hardly exist, and not starting a treatment will result in severe visual acuity loss. After the treatment is started, the patient is monitored to assess the effectiveness of the treatment. This forms the basis to continue, change or stop the treatment. However, how can we assess the effectiveness in an individual patient? One starting point is to know the expected natural course without treatment. In the case of nAMD this is well known, it will always lead to a decrease in visual acuity. Any observed improvement proves the effect of treatment in the individual patient. However, even if the visual acuity drops, it may still be true that there is an effect by slowing the visual acuity decrease. In this thesis this was studied in more detail.

We defined how data of continuous variables from RCTs can be used and analysed in a different way to decide whether a change in visual acuity can be considered an effect of the treatment. We were able to calculate the percentage of patients in whom the treatment must have had an effect for every level of change in visual acuity. We also calculated the VA at which this percentage was zero per cent. Above this level of change in visual acuity at least some patients must have benefited from this intervention. This supports the clinician in sometimes difficult decisions such as stopping or changing a treatment that is assumed to be not effective for the patient. We have given several examples of how this method can be used in other eye diseases when the outcome is a continuous variable. Other applications of the method in other fields of medicine are possible, for example to assess whether an antihypertensive drug has been effective for a patient, since the blood pressure is a continuous variable.

Further research is needed to support this method. Data from the VISION trial could be used for new data analyses to validate the method.7 In the VISION trial, pegaptanib was compared to placebo for nAMD. After one year, patients were re-randomised between placebo and pegaptanib. Based on our method, several new subgroups of patients treated with pegaptanib could be defined, based on the magnitude of change in visual acuity at one year. These subgroups could be compared in terms of the effect-modifying effect of pegaptanib versus placebo in the second year. In the subgroups defined by a visual acuity change above our cut-off at one year, there should be a higher effect after another year, 
and likely no effect in subgroups defined by visual acuity change below this level. Many attempts by several means were tried to get access to the original data but the data owners unfortunately did not give any response.

In addition to the above method we designed another approach to assess the level of visual acuity above which treatment should be continued or stopping the treatment could be discussed with the patient below this level. Costs can be saved if unnecessary treatment is prevented. However, it is hard to devise a guideline defining when to stop treating a patient. It is however important to assess which patients are expected to derive benefit from the treatment, in order to support decision-making of continuing treatment.

nAMD is a disease that does not shorten life-span but does incur a dramatic decrease in quality of life. In a value-based care context, the ultimate goal of treating nAMD is to provide patient value: to improve, or stabilise, quality of life. A decreasing level of visual acuity will lead to a decreasing level of quality of life. For example, if the visual acuity is above 0.5 , driving is still possible and allowed. A further decrease leads to a loss of driving possibilities but may still be good enough to allow reading. A further decrease will also lead to a loss of reading possibilities. Ultimately, a level of visual acuity will be reached below which the quality of life will not be affected further. Certain patients with an already very low visual acuity may not experience a deterioration of their quality of life with further deterioration of visual acuity. The question is what the cutoff point in visual acuity is. In this thesis, we developed a method to assess this point and multiple methods showed that this cut-off point was at 0.05 Snellen visual acuity.

Caution is warranted when translating this result into a guideline that affects a population as a whole. Firstly, before assuming that the visual acuity is at a level of 0.05 for a patient, it should be noted whether visual acuity did not improve any further below this threshold with the treatment and is expected not to improve with the treatment for another eye condition, for example cataract extraction. This should be assessed on a case-by-case basis. Some patients may still report a benefit after an anti-VEGF injection even though the visual acuity does not improve. Secondly, visual acuity can sometimes be a highly "volatile" measure and spontaneous variations have been noted in clinical practice. Continuous monitoring of patients, with and without treatment, remains warranted.

nAMD may not be the only eye condition in one patient. For instance, a patient can have both nAMD and glaucoma, with a low visual acuity accompanied by advanced visual field depression. In this case, quality of life may actually deteriorate if the visual 
acuity decreases below 0.05. Continuing to treat the glaucoma with advanced visual field depression and treating the nAMD with low visual acuity is needed, as it is uncertain which condition mainly contributes to the low visual acuity.

Finally, in order to balance the outcome and costs in daily practice, monitoring of patients needs to take place and data from daily practice need to be analysed. In the era of electronic patient records and Big-Data collections, continuous monitoring of processes and outcome can and should be applied to increase transparency and support value-based care. In the last chapter of this thesis, a systemic approach was described to analyse data from daily practice to assess if and how an improvement in outcome could be achieved. We used data regarding the treatment of nAMD with photodynamic therapy, ranibizumab and bevacizumab. This approach followed several steps based on elements of the process of care and the outcome of care. Process variables that were taken into account in this outcome analysis, directly determine the outcome. These involve:

(1) Severity of the disease with which the patient presents. This variable determines the prognosis of the disease, the type of intervention needed and the outcome of the intervention. We showed that improvements in outcome could be achieved if patients delay is prevented. Moreover, data on this item is needed to adjust for confounding when outcomes are compared.

(2) Ophthalmologist-dependent process variables. These include assessment as to whether the diagnosis was correct, assessment as to whether the correct indication for a treatment was made and whether the treatment or intervention was correctly conducted. We showed that improvements can be achieved in assessing the correct diagnosis. Prevention of a delay in treatment can also improve the outcome.

(3) Patient-dependent process variables regarding adherence like adherence to prescribed medication, follow-up appointments and health- or life-style advice. In the case of nAMD and treatment with anti-VEGFs, regular visits to the ophthalmologist and timely reporting of symptoms of recurrence of a choroidal neovascularisation are issues to address to improve outcome. 
Outcome analyses can be conducted in several ways. These analyses provide indications that the outcome can be improved and provide insight into which patient characteristics and process variables affect the outcome. These analyses take several steps:

(1) Choosing the relevant outcome parameter. The parameter should be directly relevant to the patient like VA, or be causally related to patient relevant outcome like the presence of a leaking choroidal neovascularisation on optical coherence tomography Moreover, the parameter should have been assessed in other comparator studies. Differences may exist in the method and criteria that are used to assess the outcome. This influences the results of the comparison.

(2) Comparing the outcome parameter with a comparator and choosing the correct comparator. Two most important approaches were the possibility of confounding by indication and the selection of a cohort that reflects the theoretical outcome without treatment, in the treated cohort.

(3) Correcting for confounding variables. A search is needed for a cause that explains a difference in outcome confounders and effect modifiers. For example, in the case of nAMD the lesion type of the choroidal neovascularisation on fluorescein angiography is important. The research question determines the relevant confounding factors.

(4) Identifying relevant patient characteristics and relevant process variables that determine the outcome. This may reveal the changes that can be made to improve the outcome. Either by improving diagnoses and indication for treatment or process variables related to the intervention.

This way of analysing contributes to the opportunities for an ophthalmologist to participate in that data analysis and discussions in the era of big data and value-based care. These discussions are inevitable since value-based care is seen as a breakthrough that will change the face of medicine, the goal of which is to lower health care costs and improve quality and outcomes. 


\section{References}

1. Lanzetta P, Mitchell P, Wolf S, Veritti D. Different antivascular endothelial growth factor treatments and regimens and their outcomes in neovascular age-related macular degeneration: a literature review. Br J Ophthalmol 2013;97:1497-1507.

2. Bell CM, Urbach DR, Ray JG, et al. Bias in published cost effectiveness studies: systematic review. BMJ 2006;332:699-703.

3. Catala-Lopez F, Ridao M, Alonso-Arroyo A, et al. The quality of reporting methods and results of cost-effectiveness analyses in Spain: a methodological systematic review. Systematic reviews 2016;5:6.

4. Brown G, Brown MM, Kertes P. Value-Based Medicine's comparative effectiveness and costeffectiveness analyses: 27 000,000 possible input variants. Evidence-Based Ophthalmology 2011;12:5253.

5. Brown MM, Brown GC. Update on value-based medicine. Curr Opin Ophthalmol 2013;24:183-189.

6. Van Gestel A, Severens JL, Webers CA, Beckers HJ, Jansonius NM, Schouten JS. Modeling complex treatment strategies: construction and validation of a discrete event simulation model for glaucoma. Value Health 2010;13:358-367.

7. Gragoudas ES, Adamis AP, Cunningham ET, Jr., Feinsod M, Guyer DR. Pegaptanib for neovascular age-related macular degeneration. N Engl J Med 2004;351:2805-2816. 

4.
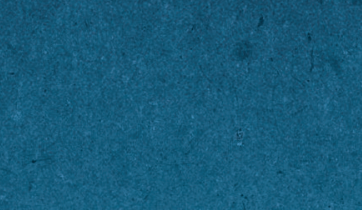

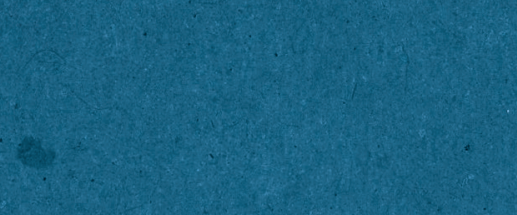
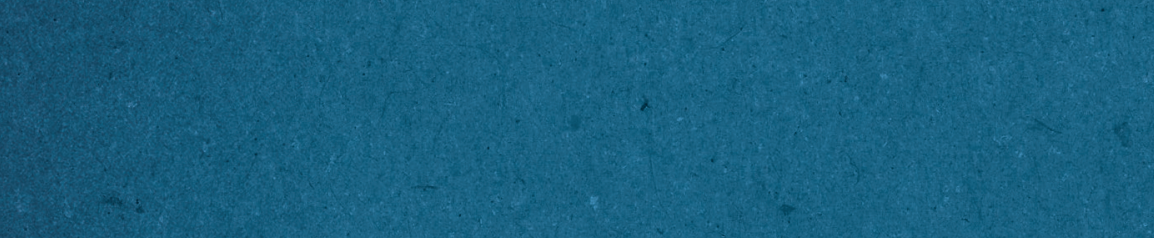

8

$y 3$

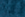




\section{Chapter 9}

Summary 


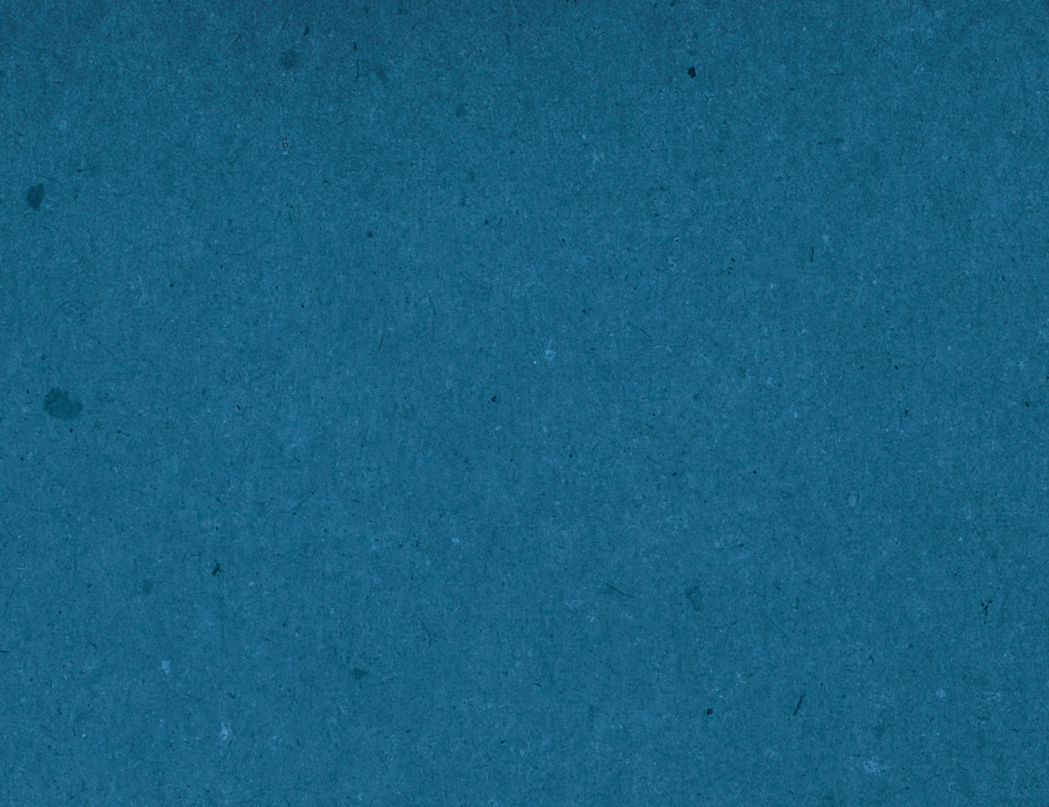




\section{Summary}

Age-related macular degeneration (AMD) is known as the leading cause of irreversible severe visual loss and blindness in the elderly in high-income countries. Neovascular AMD (nAMD), a form of AMD, was notorious for its poor prognosis when left untreated, with poor end-stage visual acuity (VA). This was the course of the disease until the availability of intravitreal anti-vascular endothelial growth factors (anti-VEGFs). These treatments are extremely effective but also expensive. Moreover, regular monitoring and frequent intravitreal injections are needed to achieve a sustained improvement in VA.

With the advance of value-based health care there is an increasing interest to consider both costs and outcome in patient care. nAMD and its treatment with its high costs, burden for health care and high efficacy make it of special interest to consider nAMD from a value-based health care perspective. This thesis addresses several topics that are of relevance in this context.

In Chapter 1, we provide an overview of nAMD as a disease and public health problem, with the treatment modalities available. In the context of value-based health care, we describe why it is important to identify the patient value and financial value for treatments for nAMD. To address these issues in relation to each other, the costs and benefits of a treatment can be assessed with cost-effectiveness analyses.

In Chapter 2, we address the issue as to whether it is of value to treat nAMD based on its natural course. We therefore describe the natural course of nAMD in a computerized model based on a literature review, combined with quality of life (QoL) data from our own patient population. In the analysis, Snellen VA decreased to 0.4 and 0.07 after 5 years in the better-seeing, and worse-seeing eye, respectively. After 4.3 years, VA was $<0.5$ in the better-seeing eye; <0.3 after 7 years; and 0.05 after 17 years. QoL score decreased from 0.6 to 0.45 after 10 years. We conclude that the natural course of nAMD in both eyes needs to be considered when informing patients, since the disease not only affects the first eye, but also in the fellow eye, which mainly defines QoL since it is most often the better seeing eye. Visual acuity data show that VA in the better-seeing eye decreases to below 0.5 in 4.3 years. Quality-of-life data show, that nAMD affects QoL significantly over time. Moreover, treatment should start when the first eye is affected, since it could become the better-seeing eye in the future. Overall, given its natural course, nAMD is a disease that is certainly worth treating. 
In Chapter 3, we consider the two dimensions of value-based health care, costs and outcome, in relation to each other. We report in a review of literature the quality, validity and usefulness of published cost-effectiveness analyses (CEA) for therapies for nAMD. We included and assessed 48 studies, of which 44 did not meet four basic model validity criteria specific to CEAs in the field of ophthalmology. These four criteria are: both eyes analysed instead of one; a time horizon extending beyond four years; extrapolating VA and treatment intervals beyond trial data realistically; and including the costs of low-vision. In two of the four studies that aligned with the quality and validity criteria, bevacizumab as-needed (PRN) was more cost-effective than bevacizumab monthly, aflibercept or ranibizumab monthly or PRN. In two studies, ranibizumab (PRN or treat and extent) was dominant over aflibercept. In two other studies, aflibercept was either more cost-effective or dominant over ranibizumab monthly or PRN. We conclude that the majority of the published CEAs are of insufficient quality and validity. These do not provide a good estimate of the value for money of these new treatments. They wrongly inform decision-makers at the possible cost of opportunities for ophthalmologists to treat patients. As such, they may negatively influence overall patient outcomes and/or societal costs. Future CEAs need to be improved and only published when they are of sufficient quality and validity.

In Chapter 4 we took the step to develop an improved cost-effectiveness model taking into account all relevant quality and validity issues as discussed in Chapter 3. We investigated the cost-effectiveness of aflibercept compared to bevacizumab, ranibizumab, and no treatment, and studied the influence of model parameters. Aflibercept has been introduced as an anti-VEGF equally effective to ranibizumab, but less costly. New costeffectiveness analyses were needed, as nAMD models used today give biased results. We developed a patient-level, VA-based, 2-eye model. We derived data on the efficacy from randomised controlled trials evaluating the outcomes of aflibercept, bevacizumab, and ranibizumab. We assessed resource utilisation in interviews with nAMD patients. We based cost estimates on standard health care cost prices. Time horizons were 2 and 5 years. We employed a societal perspective. We found that aflibercept was a more costeffective treatment for nAMD over ranibizumab. However, aflibercept is not a costeffective treatment when compared to bevacizumab. Application of inappropriate model assumptions leads to a different and biased cost-saving estimate of the cost-effectiveness of aflibercept. Cost-effectiveness analyses should be conducted with appropriate models; inappropriate models lead to a biased view on the value of anti-VEGFs.

In Chapter 5, we started with the view that any clinical decision implicitly involves costs or savings. This is especially relevant in $\mathrm{nAMD}$ were the national, Dutch guidelines state that the ophthalmologist should start with bevacizumab and change to a higher-cost 
anti-VEGF in case bevacizumab is not effective, raising the cost of treatment. This raises the question on what grounds the ophthalmologist can decide that a drug is effective. We present a new epidemiological method relying on randomised controlled trial data to assess whether a treatment was effective, aiding in the decision to continue or stop the treatment in an individual patient. We calculated a cutoff point in the change of a continuous outcome for which a proportion of treated patients achieved a change better than this cutoff point for which we could conclude with certainty that this change occurred as a result of the treatment. This cutoff point can then be applied to individual patients during routine therapy. We applied the method to data from the Minimally Classic/Occult Trial of the Anti-VEGF Antibody Ranibizumab in the Treatment of Neovascular Age-Related Macular Degeneration (MARINA), in which patients with nAMD were treated with monthly intravitreal injections of ranibizumab. We conclude that contrary to common interpretation, no change, or a limited decline in the outcome (VA), can still imply that some patients are better off with the treatment than without treatment. Stopping the treatment above the cut-off point may not be appropriate since it was effective in at least a proportion of patients. This method can be applied to a broad range of continues outcome scales and conditions.

In Chapter 6, we developed another approach that could help to decide whether to continue a treatment. We determined whether there is a level of VA in nAMD, above which the correlation of VA with disease-related QoL is significantly greater than below this level. In face-to-face interviews, we assessed QoL with the MacDQoL questionnaire. We measured VA with standardised Radner reading charts. We used regression splines analysis with a single hinge point, with the MacDQoL score as dependent and VA as independent variable. We varied the $\mathrm{x}$-coordinate (VA) of the hinge point over a wide range. The hinge point producing the best fit of the model to the data was selected. We also performed a second method of regression splines analysis, without a pre-setting of hinge points. The analyses showed that when the VA is above 0.05, there is a stronger and significant relation between VA and QoL. At 0.05 VA or lower, the relation between VA and QoL approaches zero. In case of a better VA, a difference in VA implies a significant difference in QoL. In case a poorer VA is present, a difference in VA is unlikely to imply a difference in QoL. Therefore, in treating nAMD, the aim should be to keep Snellen VA above 0.05 in order to have an impact on QoL. If it is certain that the best-corrected VA below 0.05 is permanent, these findings imply there may be less, if any, benefit to continue further treatment. This is to be evaluated on a case-by-case basis.

In Chapter 7, we propose a systematic approach to study the process and outcome of ophthalmological care in daily practice, to support the needed tools to participate in data analyses and discussions on data driven quality improvements and value-based care. We 
illustrate this approach with an evaluation of interventions for nAMD. In this cohort study, we reviewed the medical records of 473 consecutive patients with nAMD confirmed by fluorescein angiography. At each visit, VA, results from ophthalmic examination and the treatment were recorded. The steps involved were assessment of (1) the severity of the disease at presentation, (2) ophthalmologist-dependent process variables, and (3) patient-dependent process variables. Outcome analysis was performed in several steps: (1) choosing the relevant outcome parameter, (2) comparing the outcome parameter with a comparator and choosing the correct comparator, (3) correcting for confounding variables, (4) identifying relevant patient or process variables that determine the outcome. Main outcome measures were VA in logarithm of the minimal angle of resolution. We found that patient delay, diagnosis and indication for treatment and treatment conduct can be improved to improve the outcome in nAMD. Outcome studies can be conducted in several ways. This study revealed that PDT was ineffective in daily practice. Anti-VEGFs were effective and as effective as in RCTs, depending on the type of outcome analysis. Correct selection of a comparator cohort and addressing confounding, including confounding by indication and effect modification is needed to achieve valid results and interpretation. Such a systematic approach can reveal opportunities to improve the outcome of care. We conclude that a systematic evaluation of the diagnosis, the indications, the processes and outcomes of new interventions in daily practice is needed at an early stage to improve the efficiency of their application. Such a systematic approach is needed to address issues raised in the era of value-based health care. 

4.
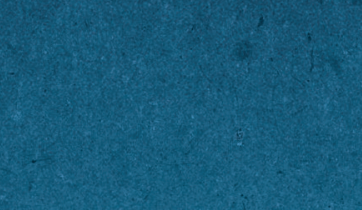

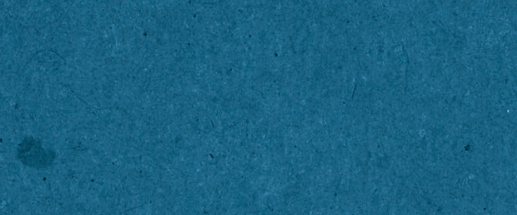
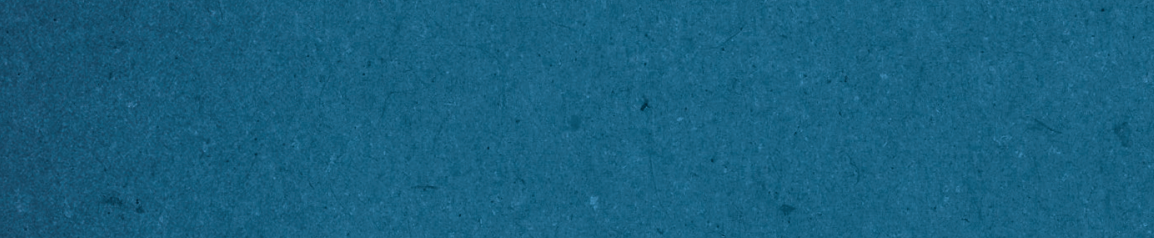

8

$y 3$

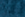


Chapter 9

Samenvatting (Summary in Dutch) 


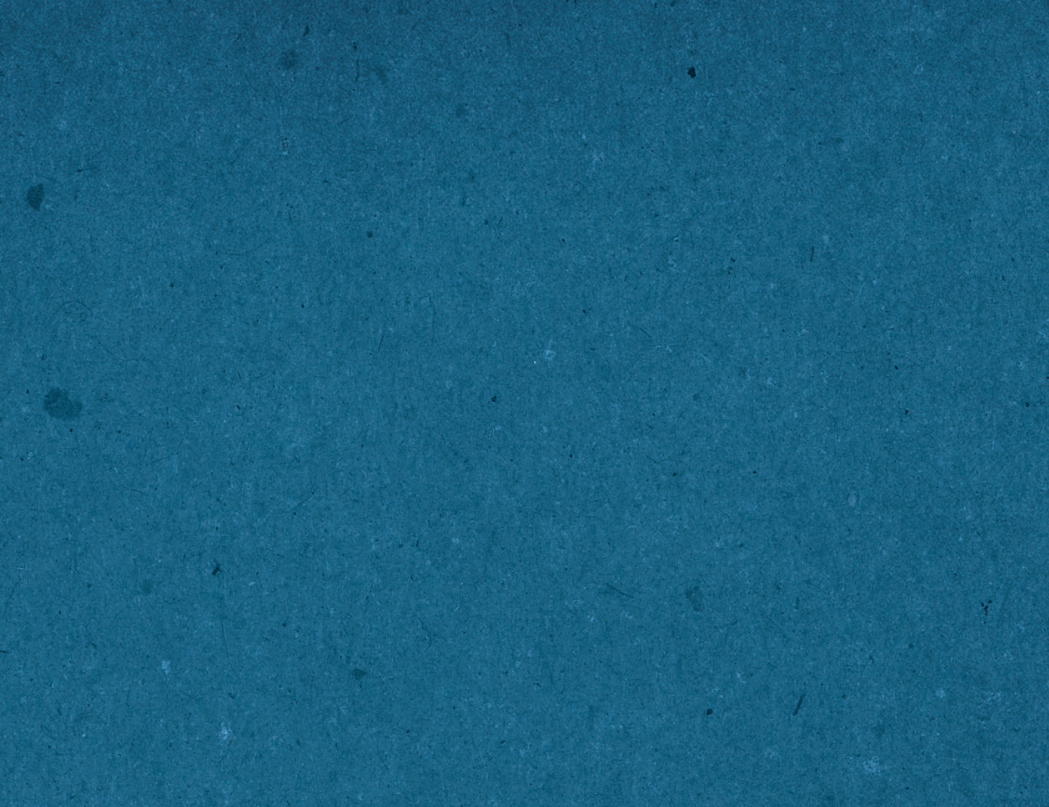




\section{Samenvatting}

Leeftijdsgebonden maculadegeneratie (AMD) is de hoofdoorzaak van irreversibele, ernstige slechtziendheid en blindheid bij ouderen in Westerse landen. Een vorm van AMD, neovasculaire leeftijdsgebonden maculadegeneratie (nAMD), had zonder behandeling een zeer slechte prognose, met blindheid tot gevolg. Dit was het natuurlijke beloop van de ziekte vóórdat de intravitreale anti-vascular endothelial growth factors (anti-VEGFs) beschikbaar kwamen. Deze medicijnen zijn zeer effectief, maar daarnaast zijn de kosten van de behandelingen hoog. Verder zijn regelmatige controle en frequente toediening van intravitreale injecties nodig, om een blijvende verbetering in het gezichtsvermogen te bewerkstelligen.

Met de ontwikkeling van value-based health care is er een toenemende aandacht voor zowel de kosten als de uitkomsten van de patiëntenzorg. Het grote aantal patiënten met nAMD, de effectieve behandeling met anti-VEGFs en de zeer goede uitkomsten, de preventie van blindheid, maar met frequente controles en hoge kosten, maken het een relevant onderwerp om vanuit een value-based health care perspectief te onderzoeken. Dit proefschrift beschrijft verschillende onderwerpen die in deze context relevant zijn.

In hoofdstuk 1 geven wij een overzicht van nAMD als ziekte en de behandelingen die beschikbaar zijn. In de context van value-based health care wordt beschreven waarom het belangrijk is om de gevolgen van een ziekte en de waarde van een behandeling voor de patient vast te stellen en de kosten van behandelingen van nAMD te berekenen. Om deze aspecten te bestuderen met betrekking tot elkaar, kunnen de kosten en uitkomsten van een behandeling worden beoordeeld met kosten-effectiviteitsanalyses.

In hoofdstuk 2 wordt besproken of het van waarde is om nAMD te behandelen, gebaseerd op het natuurlijk beloop van de ziekte. Daartoe beschrijven wij het natuurlijk beloop van nAMD met een computermodel gebaseerd op een literatuur review en gecombineerd met kwaliteit van leven data van onze eigen patiëntenpopulatie. In de analyse verslechterde de visus na 5 jaar van 1,0 naar 0,4 (Snellen visus) in het beter-ziende oog, en naar 0,07 in het slechter-ziende oog. Na 4,3 jaar was de visus in het betere oog 0,5; na 7 jaar 0,3; en na 17 jaar 0,05. De kwaliteit van leven score verminderde van o,6 naar o,45 na 10 jaar. Wij concluderen dat het natuurlijk beloop van nAMD in beide ogen in acht moet worden genomen om patiënten te informeren, aangezien de ziekte niet alleen het eerste oog treft, maar veelal ook het andere oog. Aangezien het veelal betere tweede oog de kwaliteit van leven bepaalt, zal, zonder behandeling, de kwaliteit van leven pas in een latere fase sterk afnemen. De analyse laat zien dat de visus in het beter-ziende oog afneemt tot onder 0,5 Snellen visus, de waarde waaronder geen auto meer mag worden gereden, 
in 4,3 jaar. De kwaliteit van leven analyse laat zien dat nAMD de kwaliteit van leven significant beïnvloedt. De behandeling moet dan ook worden gestart zodra het eerste oog is aangedaan, aangezien dit in de toekomst het beter-ziende en kwaliteit van leven bepalende oog kan worden. Vanwege de ernst van het natuurlijke beloop van nAMD is het behandelen van waarde, aangezien in potentie een groot effect op de kwaliteit van leven kan worden verkregen.

In hoofdstuk 3 beschouwen wij de twee dimensies van value-based health care, kosten en uitkomsten, in relatie tot elkaar. We rapporteren in een review van de literatuur de kwaliteit, de validiteit, en de bruikbaarheid van gepubliceerde kosten-effectiviteitsanalyses (KEA) voor behandelingen van nAMD. We includeerden en beoordeelden 48 studies, waarvan er 44 niet voldeden aan vier basale validiteitscriteria over de validiteit van oogheelkundige KEAs. Deze vier criteria zijn: beide ogen werden in de analyse betrokken in plaats van één oog; een voldoende lange tijdshorizon; een realistische extrapolatie van de visus en behandelingsintervallen langer dan de behandelperiode in klinische trials; en de inclusie van de kosten van slechtziendheid. In twee van de vier studies die voldeden aan de kwaliteits- en validiteitscriteria, was bevacizumab 'zo nodig' (pro re nata, PRN) meer kosteneffectief dan bevacizumab maandelijks, aflibercept, of ranibizumab maandelijks of PRN. In twee studies was ranibizumab PRN of ranibizumab treat and extend minder kostbaar en effectiever dan aflibercept. In twee andere studies was aflibercept ofwel meer kosteneffectief dan, of domineerde, ranibizumab maandelijks of PRN. Wij concluderen dat de meerderheid van de gepubliceerde KEAs van onvoldoende kwaliteit en validiteit zijn. Deze geven geen goede weergave van de waarde van deze nieuwe behandelingen per investering. Ze geven geen goede informatie aan beleidsmakers hetgeen ten koste kan gaan van de effectieve mogelijkheden voor oogartsen om patiënten te behandelen. Dit heeft dan een negatieve invloed op de maatschappelijke kosten en/of uitkomsten voor patiënten. In toekomstige KEAs moeten verbeteringen doorgevoerd worden en alleen worden gepubliceerd als zij van voldoende kwaliteit en validiteit zijn.

In hoofdstuk 4 beschrijven wij de ontwikkeling van een verbeterd kosteneffectiviteitsmodel, waarbij rekening wordt gehouden met alle relevante kwaliteits- en validiteitsvraagstukken zoals besproken in hoofdstuk 3. We hebben de kosteneffectiviteit van aflibercept vergeleken met bevacizumab, ranibizumab en "geen behandeling" en bestudeerden de invloed van verschillende modelparameters op de resultaten van de analyse. Aflibercept is geïntroduceerd als een anti-VEGF dat als even effectief als ranibizumab zou gelden, maar minder duur zou zijn. Nieuwe KEAs waren nodig, aangezien de huidige nAMD-modellen veelal geen valide resultaat geven. We ontwikkelden een 2-ogen model op patiëntniveau, met visus als uitkomst. We gebruikten gegevens over de werkzaamheid uit gerandomiseerde gecontroleerde trials waarbij de resultaten van aflibercept, bevacizumab en ranibizumab 
geëvalueerd werden. We hebben het gebruik van hulpmiddelen en -bronnen onderzocht middels interviews met nAMD-patiënten. We hebben kostenramingen gebaseerd op standaardkosten voor de gezondheidszorg en een tijdhorizon van 2 en 5 jaar gebruikt. Er werd uitgegaan van een maatschappelijk perspectief. We vonden dat aflibercept een meer kosteneffectieve behandeling voor nAMD was dan ranibizumab. Aflibercept is echter niet een kosteneffectieve behandeling in vergelijking met bevacizumab. Toepassing van onjuiste model-aannames zoals in eerdere KEAs leidt tot een onjuiste schatting van de kosteneffectiviteit, en in dit geval een onjuiste kostenbesparing met aflibercept. Kosteneffectiviteitsanalyses moeten worden uitgevoerd met passende modellen; onjuiste modellen leiden tot een onjuiste schatting van de kosten-effectiviteit van nieuwe antiVEGFs.

In hoofdstuk 5 zijn we uitgegaan van het feit dat elke klinische beslissing impliciet aanvullende kosten danwel kostenbesparingen met zich meebrengt. Dit is ook relevant bij nAMD, aangezien de nationale Nederlandse richtlijnen vermelden dat de oogarts met bevacizumab moet beginnen en pas zal overgaan op een duurder anti-VEGF, indien bevacizumab niet effectief blijkt. Dit roept de vraag op, op welke gronden de oogarts kan beslissen, dat een geneesmiddel effectief is. Wij presenteren een nieuwe epidemiologische methode die gebaseerd is op gerandomiseerde gecontroleerde trial data om te beoordelen of een behandeling effectief was, en zodoende bij te dragen aan de beslissing om de behandeling bij een individuele patiënt voort te zetten of te stoppen. We berekenden een afkappunt in de verandering van een continue uitkomstmaat, waarbij een deel van de behandelde patiënten een verandering heeft bereikt die beter is dan dit afkappunt, waarbij we moeten concluderen dat deze verandering zich bij tenminste een deel van de patiënten heeft voorgedaan als gevolg van de behandeling, omdat dit niet bereikt kan worden zonder behandeling. Dit afkappunt kan tijdens de behandeling van individuele patiënten worden toegepast. We hebben de methode toegepast op gegevens uit de MARINA trial, waarbij patiënten met nAMD werden behandeld met maandelijkse intravitreale injecties met ranibizumab. We concluderen, in tegenstelling tot de algemene interpretatie, dat 'geen verandering', of een beperkte afname van de uitkomst (visus), nog steeds kan impliceren dat sommige patiënten beter af zijn mét de behandeling dan zonder de behandeling. Het stoppen van de behandeling boven het afkappunt is dan op deze gronden niet passend, omdat het effectief was in ten minste een deel van de patiënten. Deze methode kan worden toegepast op een breed scala van continue uitkomstmaten en aandoeningen.

In hoofdstuk 6 hebben we een andere aanpak ontwikkeld die kan helpen om te beslissen om een behandeling voort te zetten. We hebben vastgesteld of er een visusniveau is bij nAMD, waarboven de correlatie van de visus met ziekteverwante kwaliteit van leven significant groter is dan beneden dit niveau. Anders gezegd, beneden een bepaald 
visusniveau heeft een verdere daling geen invloed meer op de kwaliteit van leven. In interviews aan huis hebben we kwaliteit van leven vastgesteld met de MacDQoL vragenlijst. We hebben de visus gemeten met gestandaardiseerde Radner-leeskaarten. Regression splines analyse werd gebruikt met een enkel knikpunt, met de MacDQoL score als afhankelijke en visus als onafhankelijke variabele. De x-coördinaat (visus) van het knikpunt werd gevarieerd en getest bij elke herhaling. We hebben ook een tweede methode van regression splines analyse uitgevoerd zonder een vooraf ingesteld knikpunt. Uit de analyses blijkt dat wanneer de visus hoger is dan 0,05 Snellen equivalent, er een sterkere en significante relatie is tussen visus en kwaliteit van leven. Bij een visus (Snellen) van 0,05 of lager benadert de relatie tussen visus en kwaliteit van leven nul. In het geval van een hogere visus boven 0,05 Snellen impliceert een verschil in visus een significant verschil in kwaliteit van leven. Bij slechtere visus is het onwaarschijnlijk dat een verschil in visus een verschil in kwaliteit van leven betekent. Bij het behandelen van nAMD dient daarom het doel te zijn, om de visus boven 0,05 Snellen te houden om een invloed op kwaliteit van leven te hebben. Als het zeker is dat de best-gecorrigeerde visus permanent minder dan 0,05 is, betekenen deze bevindingen dat er minder of geen voordeel kan zijn van het voortzetten van de behandeling. Dit moet per patiënt besproken en beoordeeld worden.

In hoofdstuk 7 stellen we een systematische aanpak voor, om de processsen en uitkomsten van oogheelkundige zorg in de dagelijkse praktijk te bestuderen en dataanalyses uit te voeren voor data-driven verbetering van de zorgkwaliteit en value-based care. We illustreren deze aanpak met een evaluatie van interventies voor nAMD. In deze cohortstudie hebben we de medische dossiers van 473 opeenvolgende patiënten met nAMD onderzocht. Bij elk bezoek werd de visus en de behandeling geregistreerd. De stappen voor de systematische aanpak waren de beoordeling van (1) de ernst van de ziekte bij presentatie, (2) oogarts-afhankelijke procesvariabelen, en (3) patiëntafhankelijke procesvariabelen. Uitkomstanalyses werden uitgevoerd in verschillende stappen: (A) het kiezen van de relevante uitkomstparameter, (B) het vergelijken van de uitkomstparameter met een vergelijkende behandeling en het kiezen van de juiste vergelijkende behandeling, (C) corrigeren voor confounding variabelen, en (D) het identificeren van relevante procesvariabelen die de uitkomst bepalen. De belangrijkste uitkomstmaat was visus. We vonden dat de vertraging tot het eerste contact met de oogarts, het stellen van diagnose, en de indicatie voor behandeling en uitvoering van de behandeling kunnen worden verbeterd om het resultaat bij nAMD te verbeteren. Uitkomstenanalyses kunnen op verschillende manieren worden uitgevoerd. Met deze analyse stelden wij vast dat fotodynamische therapie met verteporfin niet doeltreffend is in de dagelijkse praktijk. Anti-VEGFs waren effectief, en leken even effectief te zijn als in gerandomiseerde gecontroleerde studies, afhankelijk van het type uitkomstanalyse. De juiste selectie van 
een vergelijkingscohort en het rekening houden met confounding, inclusief confounding door indicatie en effectmodificatie, zijn nodig voor valide resultaten en interpretaties. Deze systematische aanpak biedt mogelijkheden om de uitkomst van zorg te verbeteren. We concluderen dat in een vroeg stadium een systematische evaluatie van de tijdige toegang tot de zorg, de kwaliteit van de diagnose en indicatiestelling, de processen en de uitkomsten van nieuwe interventies in de dagelijkse praktijk nodig is om de effectiviteit en efficiëntie van hun toepassing te verbeteren. Een dergelijke systematische aanpak is nodig om problemen op te sporen die bij een value-based health care benadering aan de orde moeten komen. 
4.
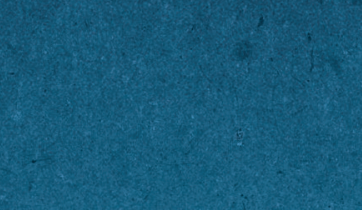

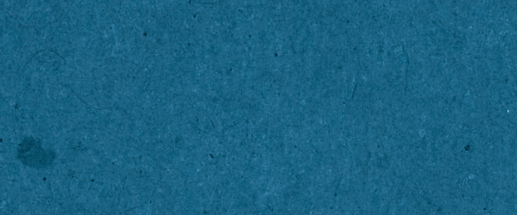
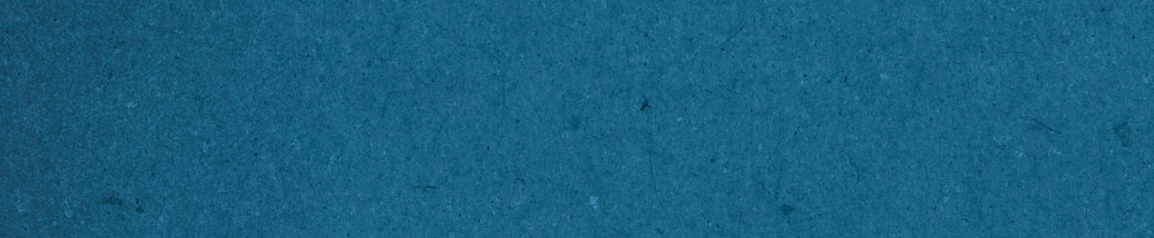

8

$y 3$

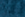




\section{Addendum}

\section{Valorisatie}

Dankwoord

Curriculum Vitae

List of publications 


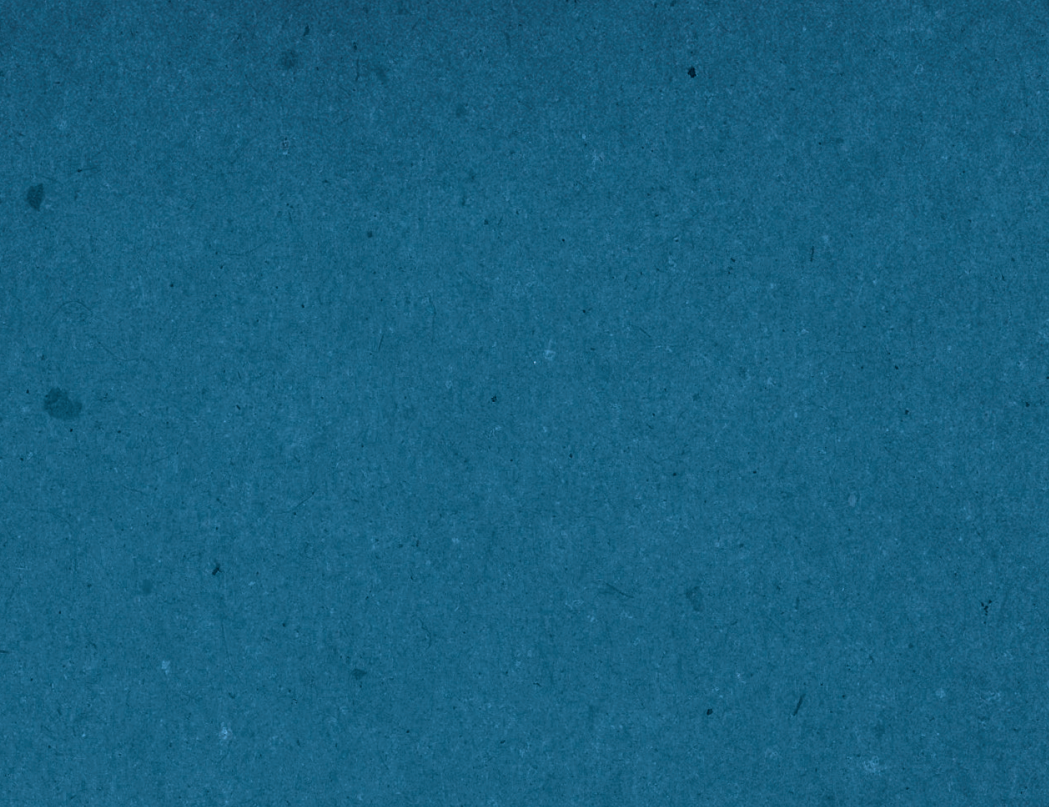




\section{Valorisatie}

Naast onderwijs en onderzoek heeft de Nederlandse overheid tevens "valorisatie" als kerntaak voor universiteiten gedefinieerd. ${ }^{1}$ Kennisvalorisatie is het proces van waardecreatie uit kennis, door kennis geschikt en/of beschikbaar te maken voor economische en/of maatschappelijke benutting en te vertalen in producten, diensten, processen en nieuwe bedrijvigheid. ${ }^{2}$ Valorisatie van wetenschappelijke kennis betekent het creëren en zichtbaar maken van mogelijkheden de opgedane kennnis in praktijk te brengen ten gunste van de maatschappij.

In het kader van dit proefschrift betreft het valorisatie-element de plaatsing van de ziekte neovasculaire leeftijdsgebonden maculadegeneratie (nAMD) in een value-based health care context. Dit betreft met name het vergroten van de kennis op het gebied van het natuurlijk beloop van nAMD, de correcte uitvoering van kosteneffectiviteitsstudies voor oogheelkundige behandelingen; de keuze, op basis van kosteneffectiviteit, tussen verschillende strategieën c.q. medicijnen voor nAMD; het bijdragen aan beslismomenten binnen de behandeling van nAMD, om zodoende over- en onderbehandeling van patiënten te voorkomen, en het beoordelen van behandelingsprocessen in de praktijk, teneinde verbeterpunten te evalueren.

\section{Maculadegeneratie}

Maculadegeneratie is een veel voorkomende aandoening waarbij er degeneratie van de macula (de "gele vlek" in de retina) optreedt. Hierdoor ontstaat er een slechter zicht en uiteindelijk, indien de ziekte niet wordt behandeld, slechtziendheid of blindheid aan beide ogen. Wereldwijd is maculadegeneratie de meest voorkomende oorzaak van blindheid bij oudere mensen. ${ }^{3}{ }^{4}$ De incidentie van de ziekte neemt toe met de vergrijzing van de bevolking. ${ }^{5}$ Wereldwijd wordt het aantal mensen met maculadegeneratie in 2020 geschat op 196 miljoen, met een toename tot 288 miljoen in $2040 .{ }^{6}$ Neovasculaire leeftijdsgebonden maculadegeneratie (nAMD) is een ernstige vorm van maculadegeneratie waarbij vorming van nieuwe bloedvaten in de macula een belangrijke rol speelt. Snelle achteruitgang van de visus is kenmerkend voor nAMD. Deze achteruitgang heeft een grote invloed op de kwaliteit van leven van patiënten met nAMD.

Tegenwoordig kan nAMD behandeld worden met medicijnen die via intravitreale injectie worden toegediend. Voor 2020 wordt het aantal van deze behandelingen van nAMD in Nederland geschat op 37.400? 
In hoofdstuk 2 onderzochten we met een literatuurstudie het natuurlijk beloop van nAMD in termen van visus en kwaliteit van leven. Bij de behandeling van ziekten is het van belang kennis te hebben van het natuurlijk beloop. De uitkomsten van dit deel van het onderzoek zijn een hulpmiddel in de (medische) praktijk, op meerdere fronten.

Een beschrijving van het natuurlijk beloop van de ziekte nAMD in de publicatie geeft een indruk van de ernst ervan weer, omdat zowel kwaliteit van leven als de snelheid waarmee deze achteruitgaat, geïntegreerd wordt weergegeven. Het is daarmee een hulpmiddel bij de priorisering van de aanpak van aandoeningen, ten behoeve van de allocatie van middelen binnen de gezondheidszorg. Een dergelijke weergave van de ernst van een ziekte in kwaliteit van leven geïntegreerd met de duur van de ziekte is een benadering zoals ook in beleidsdocumenten van de Volksgezondheid Toekomstverkenning van het RIVM wordt gehanteerd om, in combinatie met de prevalentie of incidentie, de prioritering van ziekten op populatieniveau weer te geven., ${ }^{8,9}$

Het natuurlijk beloop, zoals beschreven in deze studie, kan in de praktijk worden afgezet tegen het beloop bij behandelde patiënten. Hiermee kan de aanwezigheid en de grootte van een verondersteld effect van een behandeling worden geschat en aanwijzingen worden verkregen voor kwaliteitsverbetering. Daarmee is deze kennis goed toepasbaar in kosten-effectiviteitsanalyses.

Het beschreven natuurlijk beloop kan in de oogheelkundige praktijk worden toegepast in de voorlichting aan patiënten. Patienten met nAMD kunnen hiermee worden voorgelicht over hun prognose. Meer specifiek: over het beloop van de ziekte, indien geen behandeling zou worden gegeven. Daarbij kan het beloop op grond van deze studie niet alleen beschreven worden in mate van visusverlies maar ook in verlies aan kwaliteit van leven en het moment waarop bijvoorbeeld autorijden niet meer mogelijk is.

\section{Kwaliteit van kosteneffectiviteitsstudies}

Kosteneffectiviteitsstudies kunnen keuzes voor behandelingen ondersteunen, teneinde zoveel mogelijk waarde te creëren voor patiënten binnen beperkte budgetten. ${ }^{10}$ Hierbij is het uiteraard van belang dat kosteneffectiviteitsstudies correct worden uitgevoerd. Incorrect uitgevoerde studies leiden tot ongefundeerde beslissingen over de behandelingswijze van oogziekten in zowel patiëntenpopulaties als bij individuele patiënten.

In hoofdstuk 3 geven wij een indicatie van de kwaliteit en bruikbaarheid van de huidige kosteneffectiviteisstudies voor behandelingen van nAMD. We geven aanbevelingen over essentiële criteria waaraan kosteneffectiviteitsstudies in de oogheelkunde aan dienen te voldoen. Deze aanbevelingen zijn via de publicatie breed toegankelijk en eenvoudig toe te 
passen door auteurs. Een manuscript dient te voldoen aan bepaalde minimale vereisten voor een publicatie van een kosteneffectiviteitsstudie zoals ontwikkeld voor het BMJ door Drummond. ". Daarnaast dienen kosteneffectiviteitsstudies de toestand van beide ogen te beschouwen, een voldoende lange tijdshorizon te gebruiken, het ziektebeloop op een deugdelijke manier te extrapoleren en de kosten van blindheid en slechtziendheid te includeren. Indien deze aanbevelingen worden aangehouden in toekomstige studies, zal er een meer betrouwbare onderbouwing van keuzes in middelenallocatie beschikbaar komen. Deze aanbevelingen kunnen direct worden toegepast (gevaloriseerd) en leiden tot meer valide resultaten en conclusies. Wij hebben dit toepast bij de analyse van de kosten-effectiviteit van aflibercept.

\section{Kosteneffectieve keuze van behandelingen}

Het is van belang, zeker bij dure geneesmiddelen of behandelingen, de juiste balans te vinden tussen kosten en effectiviteit. In hoofdstuk 4 beschrijven we een kosteneffectiviteitsstudie naar de moderne behandelingen van nAMD, om de meest kosteneffectieve behandelingsstrategie te bepalen. Voor het grootste deel zijn de behandelingen met een anti-VEGF vergelijkbaar in hun effectiviteit, maar er zijn aanzienlijke verschillen in kosten. In het kader van value-based care, is waardecreatie voor de patient het einddoel. ${ }^{12}$ Waar behandelingen gelijkwaardig zijn in hun effectiviteit, wordt kostenreductie een doel om de waardecreatie te vergroten voor zoveel mogelijk patiënten. In onze kosteneffectiveitsstudie vergelijken wij verschillende van de huidige behandelingsstrategiën met elkaar en met 'geen behandeling'. Bevacizumab volgens een 'zo nodig' schema blijkt de hoogste kosten-effectiviteit te hebben. Dit resultaat ondersteunt clinici in het zoeken naar een value-based aanpak van nAMD bij hun patiëntenpopulatie.

\section{Zinnige Zorg}

$\mathrm{Na}$ de behandeling van een individuele patiënt is het belangrijk om te beoordelen of een behandeling effectief is geweest. Bij voldoende effectiviteit is daarmee voortzetting van de behandeling geïndiceerd. Als er sprake is van onvoldoende effect is voortzetten van de behandeling niet zinvol. Inzicht hierin is noodzakelijk voor de medische praktijk, conform value-based principes. Het zal toevoegen aan de waardecreatie voor patiënten, om te bepalen bij welke behandelde patiënten het mogelijk is dat zij een daadwerkelijk effectieve, c.q. zinvolle behandeling kregen. Dit beantwoordt aan de vraag in hoeverre de patiënt baat heeft bij de geleverde zorg in het kader van Zinnige Zorg.13 Krijgt de patiënt de juiste behandeling? Krijgt hij de zorg die in zijn situatie passend is, of is er sprake van over- of onderbehandeling?

In hoofdstuk 5 presenteren we een nieuwe methode om na te gaan of een behandeling effectief is geweest in een proportie van de behandelde patiënten. Hiermee is op basis 
van data van een gerandomiseerde klinische trial te bepalen bij welk afkappunt in de verbetering van de visus bij nAMD er boven deze waarde tenminste een deel van de behandelde patiënten een verbetering heeft die het gevolg is van de behandeling. Boven dit afkappunt is doorgaan met de behandeling te motiveren. Deze methode kan direct en eenvoudig worden toegepast, in de oogheelkundige praktijk, maar ook daarbuiten, bijvoorbeeld bij de behandeling van bloeddruk in de huisarts- of specialistische praktijk, om onder- en overbehandeling van patiënten te voorkomen.

Bij de bepaling van een indicatie voor behandelen wordt meestal een klinische maat gebruikt, zoals in de oogheelkunde bijvoorbeeld visus. Van overheidswege wordt het reeds gestimuleerd om in de praktijk, naast de klinische maten, meer generieke uitkomstmaten van behandelingen te meten. ${ }^{14}$ Kwaliteit van leven is een generieke maat, en vergroting van de kwaliteit van leven kan als het hoofddoel van een medische behandeling worden beschouwd.

In hoofdstuk 6 presenteren we ook een nieuw hulpmiddel in het tegengaan van overen onderbehandeling in de oogheelkundige praktijk. Kwaliteit van leven wordt bepaald door de visus die in de praktijk in belangrijke mate leidend is voor beslissingen over indicatie voor behandeling. Daar waar het niet waarschijnlijk is dat een behandeling voor de patiënt een verbetering oplevert in de kwaliteit van leven, is het mogelijk niet zinvol verder te behandelen. Een afkappunt in de visus werd bepaald, waaronder het niet waarschijnlijk is dat een verandering in visus een verandering in kwaliteit van leven betekent. Clinici kunnen dit als leidraad gebruiken in hun praktijk, met de nodige voorzichtigheid zoals beschreven in het hoofdstuk. Zo dient men er zeker van te zijn dat de visus niet meer boven het afkappunt zal komen. Hierbij moet in acht worden genomen de inherente variatie in visusmetingen, en de mogelijkeden van visusverbetering door de behandeling van andere oogziekten zoals cataract. Andere aandoeningen, bijvoorbeeld glaucoom, hoeven niet van invloed te zijn op de visus, maar wel op de kwaliteit van leven door beperking van het gezichtsveld. De waarde van dit afkappunt werd reeds gehanteerd in richtlijnen, dit onderzoek geeft zodoende onderbouwing voor huidige en nieuwe richtlijnen.

\section{De praktijk verbeteren}

Het is van grote waarde, de zorgprocessen omtrent diagnostiek, indicatiestelling en behandeling in de praktijk te beoordelen, om mogelijkheden te vinden de behandelprocessen te verbeteren. ${ }^{15}$ We leveren in hoofdstuk 7 een leidraad voor een systematische evaluatie van de zorgprocessen en uitkomsten van nAMD behandeling in de praktijk. In deze analyse waren de tijd tot het eerste contact met de oogarts, het stellen van de diagnose, en de indicatie voor behandeling, en uitvoering van de behandeling 
aangrijpingspunten om de uitkomsten van het zorgproces bij nAMD te verbeteren. $\mathrm{Na}$ toepassing van deze leidraad op onze eigen data, Stelden we vast dat fotodynamische therapie niet doeltreffend is in de dagelijkse praktijk. Anti-VEGFs waren effectief en leken even effectief te zijn als in gerandomiseerde studies. Deze vergelijking van uitkomsten geeft dan geen aanleiding te veronderstellen dat de resultaten van behandeling in de praktijk minder zijn dan het volledige potentieel. Deze systematische aanpak biedt mogelijkheden om de uitkomst van zorg te verbeteren. Verbeterpunten komen hiermee naar voren; hiermee kunnen deze verbeterpunten doelgericht worden aangepakt. 


\section{Referenties}

1. Van der Hoeven M, Rutte M. Minister en staatssecretaris van Onderwijs, Cultuur en Wetenschap. Brief aan Aan de voorzitters van de Colleges van Bestuur van de universiteiten: Valorisatie van onderzoek als taak van de universiteiten. 2005.

2. Van Drooge L, Vandeberg R, Zuijdam F, Mostert B, Van der Meulen B, Bruins E. Waardevol - Indicatoren voor Valorisatie. Technologiestichting STW, Rathenau Insitituut en Technopolis voor de Landelijke Commissie Valorisatie; 2011.

3. Klein R, Klein BE, Jensen SC, Mares-Perlman JA, Cruickshanks KJ, Palta M. Age-related maculopathy in a multiracial United States population: the National Health and Nutrition Examination Survey III. Ophthalmology 1999;106:1056-1065.

4. Bunce C, Xing W, Wormald R. Causes of blind and partial sight certifications in England and Wales: April 2007-March 2008. Eye (Lond) 2010;24:1692-1699.

5. Resnikoff S, Pascolini D, Etya'ale D, et al. Global data on visual impairment in the year 2002. Bull World Health Organ 2004;82:844-851.

6. Wong WL, Su X, Li X, et al. Global prevalence of age-related macular degeneration and disease burden projection for 2020 and 2040: a systematic review and meta-analysis. The Lancet Global health 2014;2:e106-116.

7. Keunen JE, Verezen CA, Imhof SM, van Rens GH, Asselbergs MB, Limburg JJ. [Increase in the demand for eye-care services in the Netherlands 2010-2020]. Ned Tijdschr Geneeskd 2011;155:A3461.

8. Rijksinstituut voor Volksgezondheid en Milieu. Zorg voor gezondheid. Volksgezondheid Toekomst Verkenning 2006. Bilthoven; 2006.

9. Rijksinstituut voor Volksgezondheid en Milieu. Van gezond naar beter. Kernrapport van de Volksgezondheid Toekomst Verkenning 2010. Bilthoven; 2010.

10. Zorginstituut Nederland. Kosteneffectiviteit in de praktijk. Diemen; 2015.

11. Drummond MF, Jefferson TO. Guidelines for authors and peer reviewers of economic submissions to the BMJ. The BMJ Economic Evaluation Working Party. Bmj 1996;313:275-283.

12. Porter ME, Teisberg EO. How physicians can change the future of health care. JAMA 2007;297:1103-1111.

13. Zorginstituut Nederland. Werkwijze systematische doorlichting programma Zinnige Zorg. Diemen; 2017 .

14. Kessel Pv, Triemstra M, Boer Dd. NIVEL / Zorginstituut Nederland. Handreiking voor het meten van kwaliteit van zorg met Patient Reported Outcome Measures. Utrecht; 2014.

15. Porter ME, Teisberg EO. Redefining Health Care. Boston; 2006. 




\section{Dankwoord}

Het is klaar. Ik kan terugkijken op een geweldige tijd als onderzoeker. De term "proefschrift" is treffend: het onderzoeken was niet zelden ook een echte beproeving. Gelukkig waren er veel mensen die hebben meegewerkt, gefaciliteerd, gesteund, geïnspireerd of op andere manieren hebben bijgedragen. Zonder hen was het niet gelukt, en ik wil ze hier graag voor bedanken.

Allereerst prof. dr. Carroll Webers en dr. Jan Schouten. Dankzij jullie ideeën kwam dit onderzoek tot stand en kreeg ik de gelegenheid mee te werken aan de uitvoering. Jullie hebben talloze malen de artikelen doorgenomen en eindeloos waardevolle en gedetailleerde input gegeven. Ik ben erg dankbaar voor de begeleiding en het in mij gestelde vertrouwen.

Prof. dr. Fred Hendrikse, die mij heeft aangenomen voor de opleiding tot oogarts en dankzij wie ik de gelegenheid kreeg met het onderzoek te beginnen.

Margriet van der Reis, met wie ik samen aan het onderzoek heb gewerkt. Bedankt voor jouw enorme inzet voor het onderzoek en de artikelen. Je bent een kundige top-collega.

Dr. Yvonne De Jong-Hesse, dr. Tos Berendschot en dr. Ellen La Heij, voor hun grote hulp als medeauteur. Dr. Frans Lion voor het mede-beoordelen van fluoresceïne angiografieën.

De beoordelingscommissie, voor het bestuderen van het manuscript: prof. dr. A.E.R.C.H. Boonen, prof. dr. M.A. Joore, prof. dr. J.E.E. Keunen, dr. G.G. van Merode, en dr. F.D. Verbraak.

Alle ziekenhuizen en oogheelkundige afdelingen die hun medewerking hebben verleend aan onze dataverzameling: Universiteitskliniek voor Oogheelkunde Maastricht, CatharinaZiekenhuis Eindhoven, VU Medisch Centrum Amsterdam, en ZorgSaam ziekenhuis Zeeuws-Vlaanderen.

Alle patiënten die meewerkten aan het kwaliteit-van-leven en kostenonderzoek, voor hun tijd, openheid en gastvrijheid.

De docenten van de afdeling Epidemiologie, voor hun inspirerende colleges en workshops.

Alle collega's op de onderzoeksafdeling van de Universiteitskliniek voor Oogheelkunde, tijdens mijn onderzoeks- en opleidingsjaren, voor de kleinere en grotere helpende hand bij het promotie-onderzoek, voor de gezelligheid en saamhorigheid, al dan niet na werktijd. De gehele staf van de Universiteitskliniek voor Oogheelkunde, voor mijn klinische en 
wetenschappelijke opleiding, hun waardering en hun interesse in mijn onderzoek.

Alle oogartsen in opleiding voor de saamhorigheid en werksfeer in de assistentengroep tijdens mijn promotieonderzoek en opleiding tot oogarts.

Het secretariaat van de Universiteitskliniek voor Oogheelkunde voor hun belangrijke ondersteuning bij de administratieve kant van mijn promotieonderzoek.

Mijn collega's en medewerkers van onze afdeling oogheelkunde van het st. Anna Ziekenhuis in Geldrop. Bedankt voor de top-sfeer en jullie hart voor onze afdeling. Voor jullie inzet en begrip, terwijl ik de eindsprint trok naar de promotie.

Een aantal mensen in het bijzonder wil ik bedanken voor hun bijdrage aan de ontwikkeling van dit onderzoek. Dr. Martien van Dongen, voor de begeleiding bij het afstuderen voor de master Epidemiologie. Dr. Roel Erckens, voor de begeleiding in het optisch laboratorium: mijn eerste stappen in wetenschappelijk onderzoek. Dr. Aukje van Gestel, voor haar hulp bij het leggen van het fundament van het kosteneffectiviteitsmodel. Ing. Astrid Hacking, voor het faciliteren van onderzoek doen tijdens mijn opleiding tot oogarts. Mireille van Helden, voor het stroomlijnen van de eindfase van mijn opleiding tot oogarts.

Een aantal mensen in het bijzonder wil ik bedanken voor hun bijdrage aan de ontwikkeling van mijzelf als onderzoeker. Frank van den Biggelaar, voor je doorvragen en je hoge tolerantie voor mijn slechte kantoorhumor. Jacqueline Counotte, voor haarverf-, golden power- en maanlichtfeesten. Ali al Dulaimi. Bedankt voor je eindeloze positivisme, je inspiratie om avonturen aan te gaan, niet bang te zijn, en om het beste uit mezelf en het leven te halen. Nordin Hanssen. Gouden bellen! Samen hebben we muziek gemaakt, toneel gespeeld, en snackbarpoëzie uitgevonden. Jouw fijnzinnige woorden in spannende tijden zijn onmisbaar Paul van der Lee, jij ondoorgrondelijk fenomeen. Bedankt dat ik je heb leren kennen, van obscure absint-filmavonden tot het onveilig maken van Zuid-Frankrijk. Lot Sewing, ergens lijken wij heel erg op elkaar. Dankjewel voor onze bijzondere vriendschap. Thijs Vaessen, voor je steun en je voorbeeld voor mij als assistent, als oogarts, en allround goeie kerel. Rob van der Veen, bedankt voor jouw gastvrijheid, ik hoop er nog vaak gebruik van te kunnen maken. En voor je scherpe geest en bovennatuurlijk scherpe blik.

Mijn lieve ouders, voor het grootbrengen twee prachtkinderen, voor een mooie en inspirerende jeugd en een buitengewone opvoeding. Voor de grandioze steun en een eindeloos geloof in mij. 
Franke en Josef, bedankt voor jullie enthousiasme voor elk van mijn nieuwste verhalen en ontdekkingen, en voor mij laten beseffen dat werk en onderzoek nog maar een deel van het grotere avontuur is.

Liefste Juliëtte, jij inspireert mij om goed te doen, tot het maken van het allerbeste en het beleven van het allermooiste. Jij hebt de hoogte- en dieptepunten van dichtbij gezien, altijd een rotsvast vertrouwen gehad in mij en dat het me zou gaan lukken. Dankjewel. 



\section{Curriculum Vitae}

Mari Elshout was born in Eindhoven, The Netherlands, on 3 April 1985. He graduated from the Gymnasium at Lorentz Casimir Lyceum in Eindhoven in 2003. In the same year, he started his medical studies in Maastricht. Here, he also attended the Academy of Fine Arts in 2004 and 2005. In 2006, he worked as a student-assistant in research projects in cardiology, for dr. R. Tieleman at the Maastricht University Medical Center, and for dr. R. Michels, at the Catharina-Hospital Eindhoven. He participated in the International Track in Medicine with internships in the United Kingdom, South Africa, India, and Belgium. During his internships, he became interested in ophthalmology and spent a research internship under the supervision of dr. R.J. Erckens and dr. J.S.A.G. Schouten at the University Eye Clinic in Maastricht. He completed a senior clinical internship in Internal Medicine / Endocrinology in Maastricht, supervised by dr. R.J.M.W. Rennenberg and prof. dr. N.C. Schaper. After obtaining his medical degree in 2009, he started a research fellowship, which led to the current thesis, under the supervision of prof. dr. F. Hendrikse, prof. dr. C.A.B. Webers, and dr. J.S.A.G. Schouten at the University Eye Clinic in Maastricht. During his research, he obtained a second Master's degree, in Epidemiology, at Maastricht University, in 2011. From 2011 to 2016, he was a resident in ophthalmology at the University Eye Clinic. In 2016, he worked as an ophthalmologist in Weert and Heerlen. He currently works as an ophthalmologist in St. Anna Hospital in Geldrop. He lives in Eindhoven with his wife, Juliëtte, and their son, Kobus. 



\section{List of publications}

\section{Related to this thesis}

Elshout M, Van der Reis MI, Webers CA, La Heij EC, Hendrikse F, Schouten JS. A new epidemiological aid in deciding whether to continue or stop a treatment. Investigative Ophthalmology and Visual Science. 2012 Jul 1;53(8):4331-4336.

Elshout M, Van der Reis MI, Webers CAB, Schouten JSAG. The cost-utility of aflibercept for the treatment of age-related macular degeneration compared to bevacizumab and ranibizumab and the influence of model parameters. Graefe's Archive for Clinical and Experimental Ophthalmology. 2014 Dec;252(12):1911-1920.

Elshout M, Van der Reis MI, de Jong-Hesse Y, Webers CAB, Schouten JSAG. Distinguishing between better and worse visual acuity by studying the correlation with quality of life in neovascular age-related macular degeneration. Ophthalmology. 2016 Nov;123(11):2408-2412.

Elshout M, Webers CAB, Van der Reis MI, de Jong-Hesse Y, Schouten JSAG. Tracing the natural course of visual acuity and quality of life in neovascular age-related macular degeneration: a systematic review and quality of life study. BMC Ophthalmology. $2017 \mathrm{Jul}$ 11;17(1):120.

Elshout M, Webers CAB, Van der Reis MI, Schouten JSAG. A systematic review on the quality, validity and usefulness of current cost-effectiveness studies on the treatment for neovascular age-related macular degeneration. Acta Ophthalmology. 2018 Dec;96(8):770-778.

\section{Beyond the scope of this thesis}

Elshout M, Erckens RJ, Webers CAB, Beckers HJ, Berendschot TT, de Brabander J, Hendrikse F, Schouten JSAG. Detection of Raman spectra in ocular drugs for potential in vivo application of Raman spectroscopy. Journal of Ocular Pharmacology and Therapeutics. 2011 Oct;27(5):445-451. 
